\title{
Electromagnetic nondestructive inspection of aircraft structures by using a magnetic flux leakage method
}

lyad Mahmood Ali Muslih

West Virginia University

Follow this and additional works at: https://researchrepository.wvu.edu/etd

\section{Recommended Citation}

Muslih, lyad Mahmood Ali, "Electromagnetic nondestructive inspection of aircraft structures by using a magnetic flux leakage method" (2005). Graduate Theses, Dissertations, and Problem Reports. 2734. https://researchrepository.wvu.edu/etd/2734

This Dissertation is protected by copyright and/or related rights. It has been brought to you by the The Research Repository @ WVU with permission from the rights-holder(s). You are free to use this Dissertation in any way that is permitted by the copyright and related rights legislation that applies to your use. For other uses you must obtain permission from the rights-holder(s) directly, unless additional rights are indicated by a Creative Commons license in the record and/ or on the work itself. This Dissertation has been accepted for inclusion in WVU Graduate Theses, Dissertations, and Problem Reports collection by an authorized administrator of The Research Repository @ WVU. For more information, please contact researchrepository@mail.wvu.edu. 
Electromagnetic Nondestructive Inspection of Aircraft Structures by Using a Magnetic Flux Leakage Method

Iyad Mahmood Ali Muslih

Dissertation submitted to the College of Engineering and Mineral Resources

at West Virginia University in partial fulfillment of the requirements

for the degree of

Doctor of Philosophy

In

Mechanical Engineering

James Smith, Ph.D., Chair

Kenneth Means, Ph.D.

Muhammad Choudhry, Ph.D.

Roy Nutter, Ph.D.

Victor Mucino, Ph.D.

Department of Mechanical and Aerospace Engineering

\author{
Morgantown, West Virginia \\ 2005
}

Keywords: Magnetic Field Modeling, Nondestructive Evaluation Test, Aircraft Aging, Inverse Problem

Copyright 2005 Iyad M. Muslih 


\title{
ABSTRACT \\ Electromagnetic Nondestructive Inspection of Aircraft Structures by Using a Magnetic Flux Leakage Method
}

\author{
Iyad Mahmood Ali Muslih
}

Aging of aircraft structures is mostly associated with fatigue cracking, de-bonding and corrosion. Detection and characterization of the structural defects at the initiation stages makes it a great challenge for any inspection technology. This study proposes a new solution for the nondestructive evaluation problem by using a magnetic flux method for non-ferromagnetic materials and provides a new neural network tool that predicts crack profiles in three dimensions by solving the inverse problem, where available neural networks can solve it in two dimensions only.

The discontinuity resulting from a crack produces disturbance to the distribution of electrical current density in the structure and as a result the magnetic field around the crack will change. The magnitude of the disturbance is determined by the size and shape of the crack. Therefore, it is possible to evaluate the crack area by magnetic field measurements. The magnetic fields from the plate edges and the wires that carry the current are very strong compared to the magnetic field produced by the crack. A new plate, called a dummy plate, is used to minimize the effect of the magnetic fields produced by the plate edges. This study proves the effectiveness of the dummy plate and shows the measurable change in the magnetic signal around the crack.

As a result of this work, a tool is now available that can solve the nondestructive evaluation problem and the inverse problem in three dimensions and has the capability to provide an enhanced assessment tool for judgment and decision-making which will improve the safety of metallic structures and save people lives. 


\section{ACKNOWLEDGEMENTS}

This dissertation would not have been possible without the help and support of several people. I would like to thank my advisor, Dr. James Smith, for his guidance, advice and full support during the course of my graduate studies, his indispensable contributions and special attention will never be forgotten. I would also like to thank my committee members, Dr. Roy Nutter, Dr. Kenneth Means, Dr. Mohammed Choudhry and Dr. Victor Mucino, for their guidance and support. I am especially grateful to Dr. Mohammed Choudhry for his help in supervising the experimental part of this study and providing the necessary instruments.

My appreciation goes to Dr. Wathiq Abdul Razzaq with the Department of Physics at West Virginia University for his invaluable discussions. I would also like to thank all my friends for their support and especially Diaa Nassar for his help with MATLAB ${ }^{\circledR}$ Software and valuable discussions in the neural network field.

Finally, and most importantly, I would like to thank my parents and my wife for their unwavering support during my long career as a student. They have always pushed me to attain higher goals, and my pursuit of a $\mathrm{PhD}$ would not have been possible without them. 


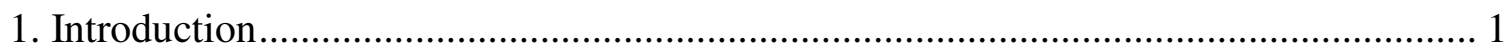

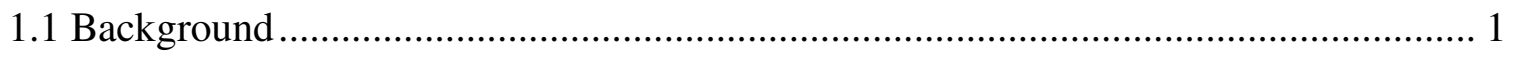

1.2 Aircraft Aging ..................................................................................................... 4

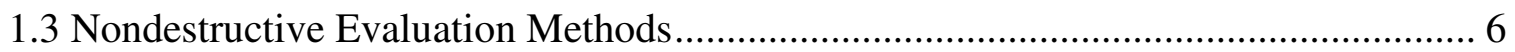

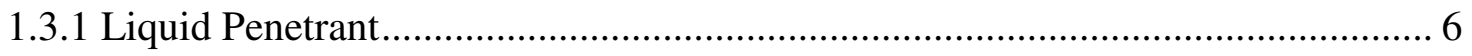

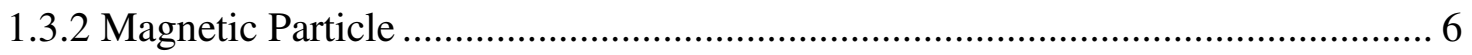

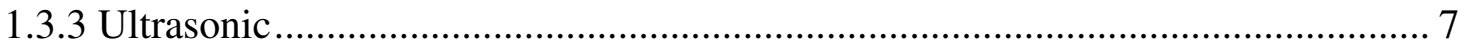

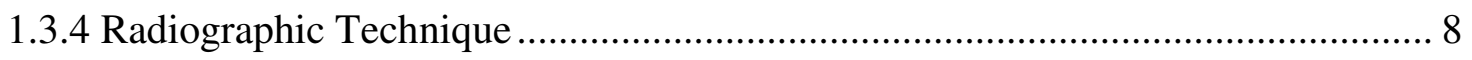

1.3.5 Acoustic Emission Technique ………………............................................... 8

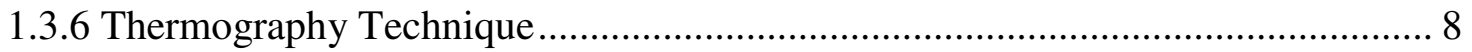

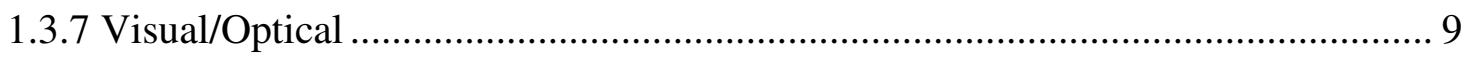

1.3.8 Sonic /Resonance ............................................................................................ 9

1.4 Inverse Problem in Nondestructive Evaluation Test ................................................. 10

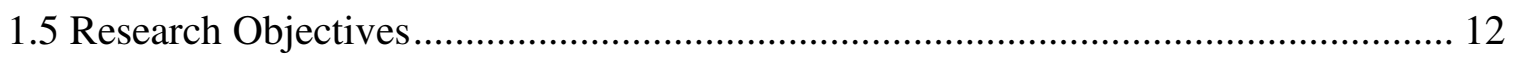

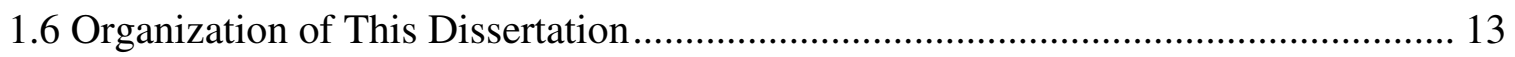

2. Electromagnetic Nondestructive Evaluation Method .................................................... 16

2.1 Concepts of Electromagnetism ............................................................................ 16

2.2 Electromagnetic Nondestructive Evaluation Methods .............................................. 22

2.2.1 Electrical Potential Drop Method .................................................................... 22

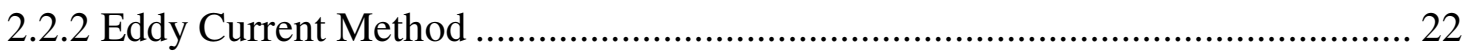

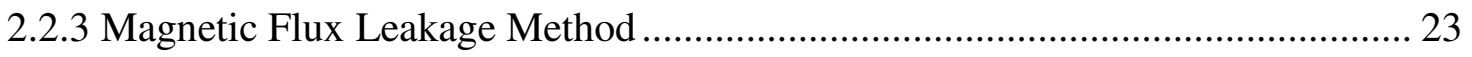

2.3 Magnetic Flux Leakage Theory …………………………................................... 25

3. Research Approach and Methodology …………………........................................ 29

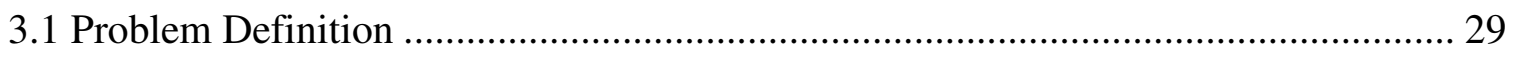

3.2 Research Approach ......................................................................................... 30

3.2.1 Mechanical and Electrical Analysis................................................................... 33

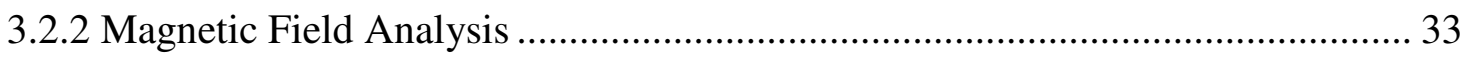

3.2.3 Neural Inverse Problem Approach ..................................................................... 34

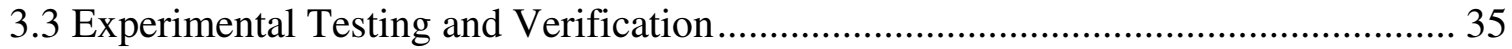




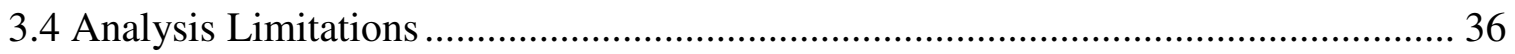

4. Mechanical and Electromagnetic Analysis .............................................................. 38

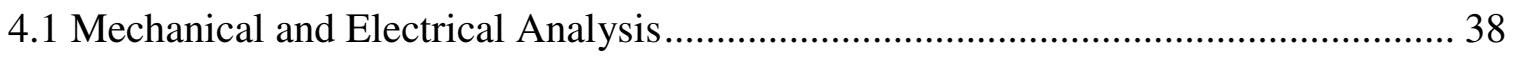

4.2 Crack Detection by Using Magnetic Flux Leakage Method ...................................... 43

4.2.1 The Magnetic Field from a Plate without a Crack ................................................ 43

4.2.2 The Magnetic Field from a Plate with a Crack ................................................... 47

4.2.2.1 Effect of the Current Flowing Thru the Plate …………………………….... 54

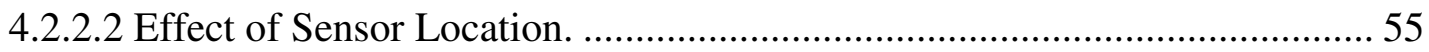

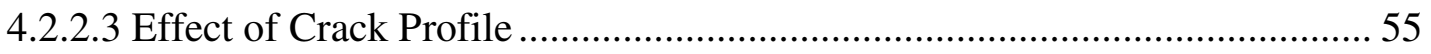

4.3. Magnetic Signal Enhancement for Better Detection .............................................. 66

4.3.1 Magnetic Fields Produced by the Wires .............................................................. 66

4.3.2 Magnetic Fields Produced by the Plate Edges................................................... 70

4.3.2.1 Effect of $t_{e}$ on the New Magnetic Field ...................................................... 71

4.3.2.2 New Analysis after Minimizing the Effect of Plate Edges .......................... 72

4.4 Method Capability ............................................................................................ 77

5. Neural Network Analysis and Solving the Inverse Problem ...................................... 83

5.1 Neural Network Modeling ..................................................................................... 83

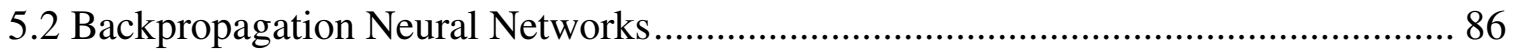

5.3 Feedforward Networks and Neuron Models ............................................................ 87

5.4 Magnetic Filed Neural Network Analysis ................................................................ 91

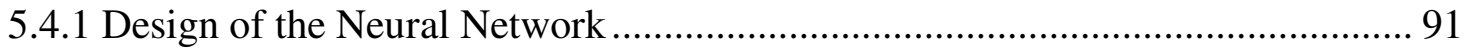

5.4.2 Neural Network with One Hidden Layer........................................................... 92

5.4.3 Neural Network with Two Hidden Layers .......................................................... 96

6. Experimental Results and Verification .................................................................. 100

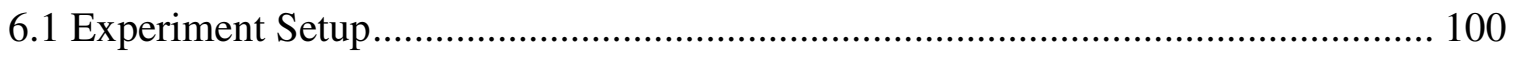

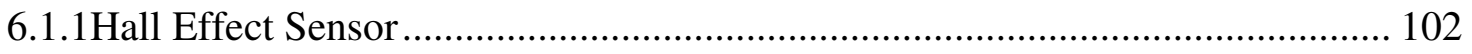

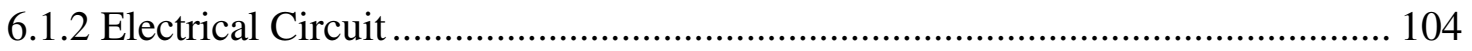

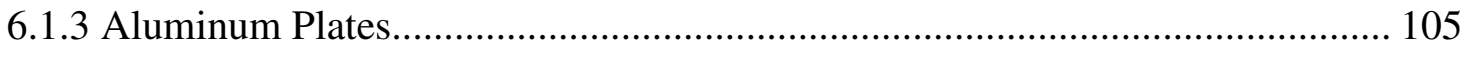

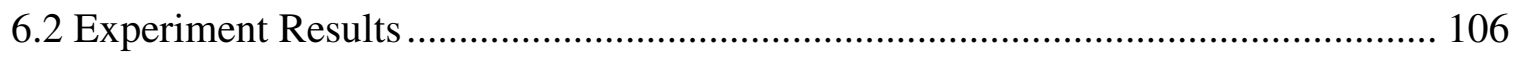

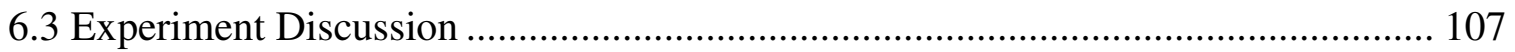

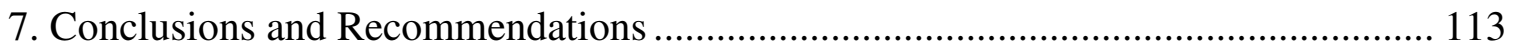




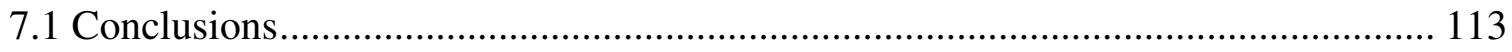

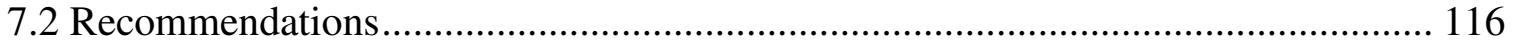

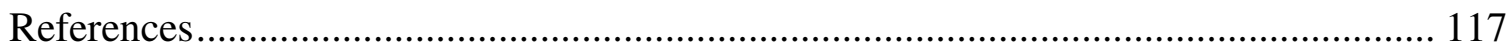

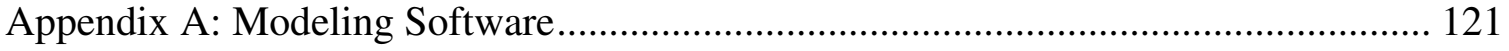

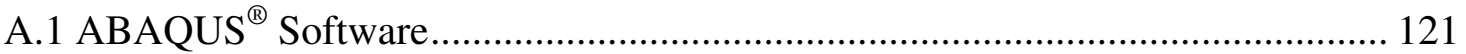

A.2 MATLAB ${ }^{\circledR}:$ Neural Network Tool Box …..................................................... 122

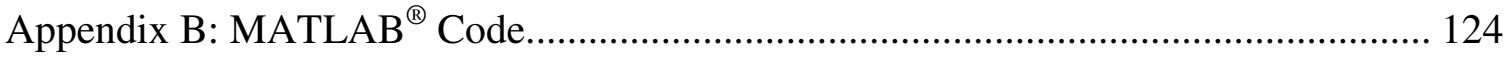




\section{List of Tables}

Table 1 Magnetic properties of some common materials [24][25].............................. 21

Table 2 Crack profiles for modeling very small cracks, dimensions in mm. ................. 77

Table 3 Neural Network results for predicting crack profiles ................................... 99

Table 4 Magnetic field experimental results at certain points around the cuts............... 107 


\section{List of Figures}

Figure 1 Basic automated system for NDE methods ................................................... 3

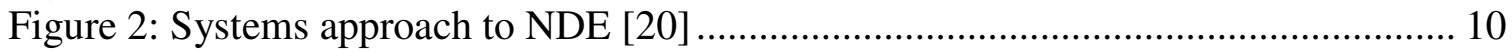

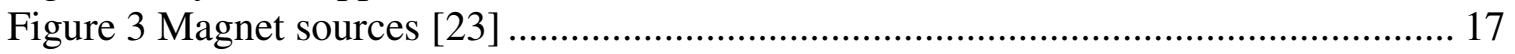

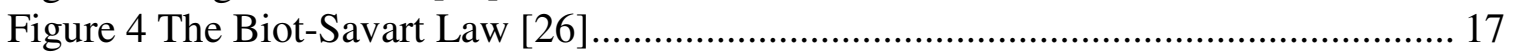

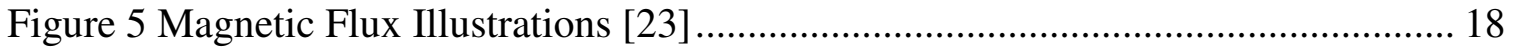

Figure 6 Plate under consideration ........................................................................ 25

Figure 7 Concept of the research methodology ....................................................... 31

Figure 8 Proposed approach for solving the NDE Problem ...................................... 32

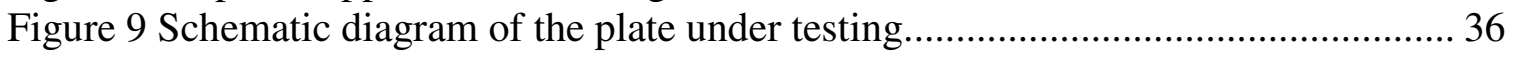

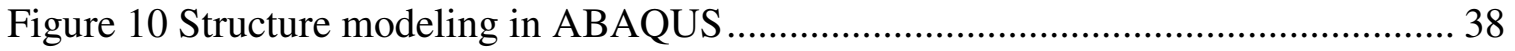

Figure 11 Structure modeling in ABAQUS using symmetry ...................................... 39

Figure 12: Electrical Current Density of the crack before applying a mechanical load ... 40

Figure 13 Stress filed around the crack tip. ............................................................ 41

Figure 14 Strain field around the crack tip ........................................................... 41

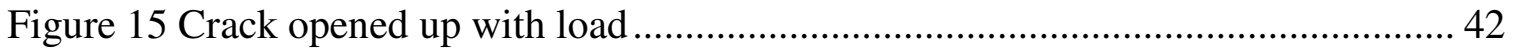

Figure 16 Plate without a crack or defect ........................................................... 44

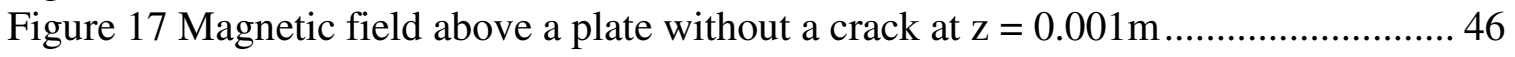

Figure 18 Magnetic field contour lines above the middle of the plate with no crack...... 47

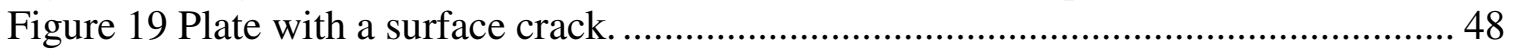

Figure 20 Plate with a crack or defect in the center................................................... 51

Figure 21 Magnetic field above a plate with a $(0.006 \times 0.002 x .001)$ meter dimensions

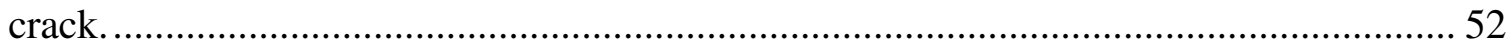

Figure 22 Close up look at the field above the crack............................................... 52

Figure 23 Magnetic field contour lines around the crack location ............................... 52

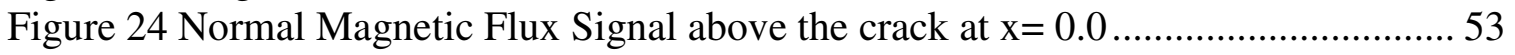

Figure 25 Effect of the current flowing thru the plate. ............................................... 54

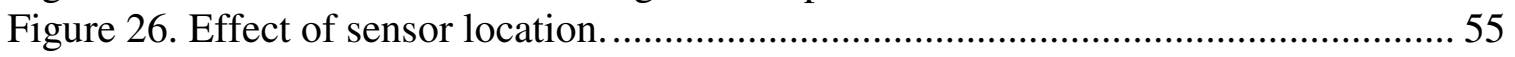

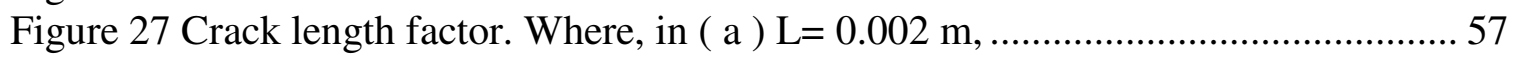

Figure 28 Magnetic field contour lines around the crack location. Where, in (a ) $\mathrm{L}=$

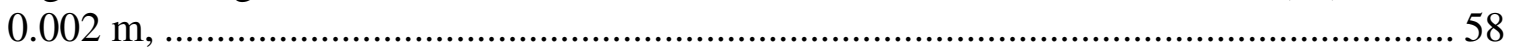

Figure 29 Crack length effect on the magnetic field at the crack tip ............................. 59

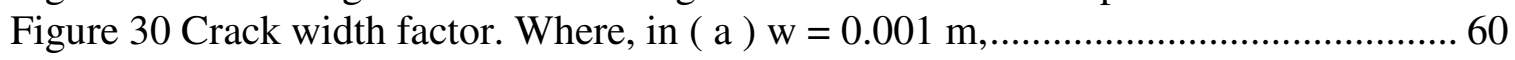

Figure 31 Magnetic field contour lines around the crack location. Where, in $(a) w=$

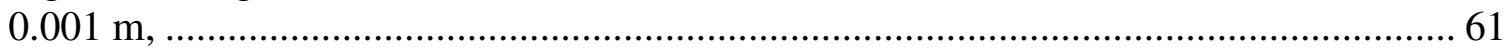

Figure 32 Crack width effect on the magnetic field at the crack tip............................. 61

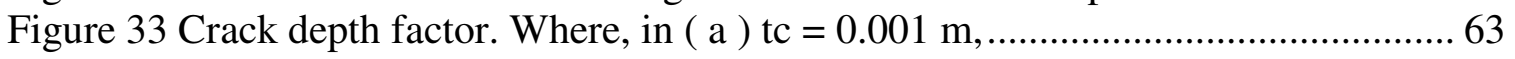

Figure 34 Magnetic field contour lines around the crack location. Where, in ( a ) tc =

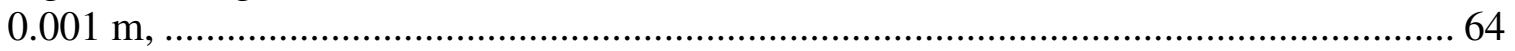

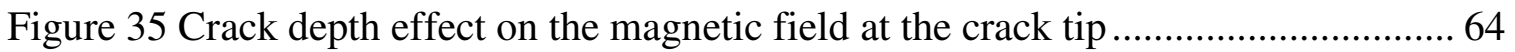

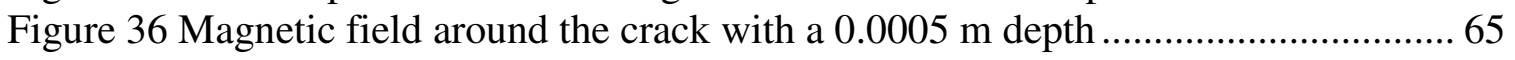

Figure 37 Magnetic field around the crack with a $0.003 \mathrm{~m}$ depth .................................. 65

Figure 38 Magnetic fields around the plate when the wires are connected in parallel to the

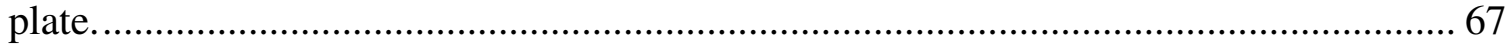


Figure 39 Magnetic fields around the plate when the wires are connected normal to the

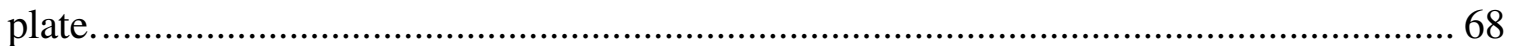

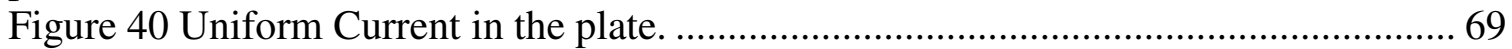

Figure 41 Current distribution according to figure (32) ................................................... 69

Figure 42 Illustrative diagrams for both the defected plate and the dummy plate ........... 71

Figure 43 Effect of $t_{e}$ on the new magnetic field. ...................................................... 71

Figure 44 3D Magnetic Filed around the same Crack. (a) Without the D. plate. (b) using

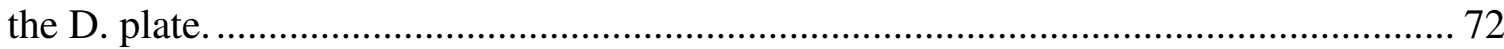

Figure 45 Magnetic signal profile around same the crack. (a) Without the D. plate. (b)

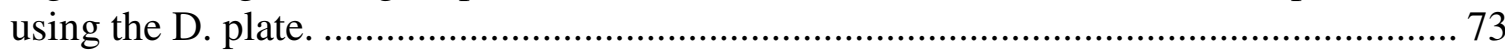

Figure 46 3D Magnetic field for crack with $0.0005 \mathrm{~m}$ depth. Where, (a) without D. plate

Figure 47 Magnetic signal profile around a crack with $0.0005 \mathrm{~m}$ depth. Where, (a)

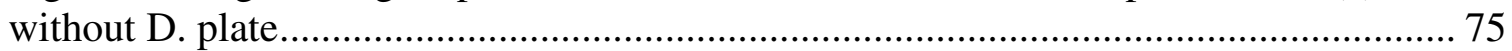

Figure 48 Crack length effect on the magnetic field at the crack tip with using the D. plate .75

Figure 49 Crack width effect on the magnetic field at the crack tip with using a D. plate76 Figure 50 Crack depth effect on the magnetic field at the crack tip with using a D. plate76 Figure 51 Magnetic field around a crack with $0.001 \mathrm{~m}$ in length and $0.0001 \mathrm{~m}$ in width.. 78 Figure 52 Magnetic field around a crack with $0.001 \mathrm{~m}$ in length and $0.00001 \mathrm{~m}$ in width.79 Figure 53 Magnetic field around a crack with $0.001 \mathrm{~m}$ in length and $1 \mu \mathrm{m}$ in width. ....... 80 Figure 54 Magnetic field around a crack with $0.0001 \mathrm{~m}$ in length and $1 \mu \mathrm{m}$ in width. ..... 81 Figure 55 A schematic diagram for a biological neuron [21] ......................................... 85

Figure 56 Schematic diagrams for an artificial neuron [21] .......................................... 85

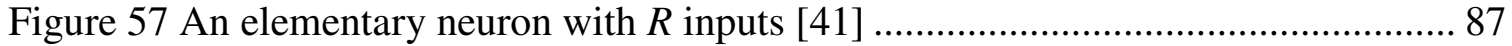

Figure 58 Two transfer functions used in backpropagation neural network analysis [42] 88

Figure 59 Multiple layers of neurons with nonlinear transfer functions [43][44] ............ 89

Figure 60 Multiple layers of neurons with one hidden layer and linear output function.. 89

Figure 61 Neural network flow chart design. ............................................................. 92

Figure 62 A neural network that has 14 neurons in one hidden layer .............................. 93

Figure 63 Training, validation and testing process for Net. No. 1.................................. 93

Figure 64 Median Error in predicting crack dimensions, Red for the length. Green for

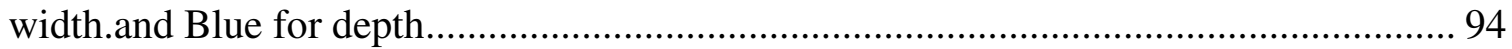

Figure 65 A neural network that has 25 neurons in one hidden layer .............................. 95

Figure 66 Training, validation and testing process for Net. No. 2.................................. 95

Figure 67 A neural network trained by two sample data libraries................................... 96

Figure 68 Training, validation and testing process for Net. No. 3 (a) for 121 library. (b)

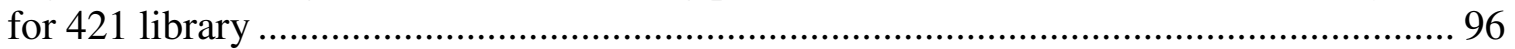

Figure 69 Neural Network with two hidden layers (20 and 30) neurons.......................... 97 Figure 70 Training, validation and testing process for Net. No. 1 with two hidden layers

Figure 71 Neural Network with two hidden layers No. 2 …………………………..... 98

Figure 72 Training, validation and testing process for Net. No.2 with two hidden layers 98

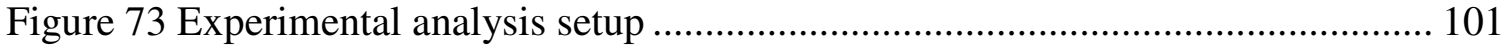

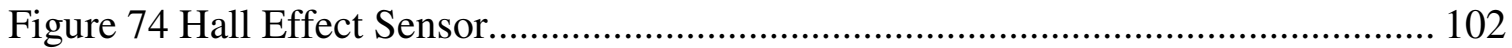

Figure 75 Experimental setup: Plate without cut at the center ....................................... 105 
Figure 76 Experimental setup: Plate with a defect at the center................................ 106

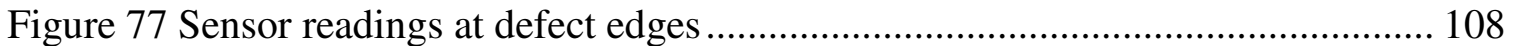

Figure 78 Magnetic fields corresponding to experiment No 2 .................................. 108

Figure 79 Magnetic fields corresponding to experiment No 3 ................................... 109

Figure 80 Magnetic fields corresponding to experiment No 4 ................................... 109

Figure 81 Magnetic fields corresponding to experiment No 5 .................................... 109

Figure 82 Magnetic fields corresponding to experiment No 6 ................................... 110

Figure 83 A comparison between the experiment and modeling results ...................... 110

Figure 84 Percentage errors between the sensor and modeling results. ........................ 111 


\section{Introduction}

The nondestructive detection and evaluation (NDE) of cracks (or crack-like defects) are highly desirable from the view point of safe and economic operation of many industries such as the aircraft industry and power generation stations. Fatigue cracks or other pre-existing cracks could lead to sudden failure of many structural components, which may carry the risk of the loss of life as well as hardware in case of catastrophic failure.

\subsection{Background}

Major components in most aircraft are made from metallic materials such as aluminum alloys or high strength super alloys. Examples of components in which this type of metal failure may occur are aircraft frame structures, fuselage and turbine blades. Many such systems are operating even beyond their design lifetime, which requires more than the originally prescribed inspection cycles.

In nondestructive inspection of aircraft structures, there are two levels at investigation: one is the detection of flaws and the other is the evaluation of flaw size and shape for predicting fracture behavior [1].

Recent researchers are interested more in electromagnetic methods than in conventional methods like ultrasonic inspection techniques [2]. It is proposed in this work that cracks can be detected and sized by electromagnetic means and then with numerical modeling this information can be used to simulate the electromagnetic field around the crack providing useful knowledge about the responding signals. Intelligent algorithms can then be developed from these models and implemented in practical instruments for the detection and sizing of cracks. 
In this work, a new electromagnetic nondestructive evaluation method is proposed by measuring the magnetic flux leakage on the top of a conducting plate with a crack. The method is a magnetic flux leakage method applied to non-ferromagnetic structures that is usually used in the aircraft industry. The magnetic field distribution on the top of a conducting plate can be calculated by considering the current density distribution in the plate [3].

At the time of inspection, the computer system should be able to visualize the magnetic field measured by the sensor at the surface of the structure, including magnetic field lines and magnetic field intensity. From this information, the computer system can provide the description of the crack profile that would generate such a distribution of magnetic flux. This description of the crack profile is obtained by solving the inverse problem. The computer program is generating a description of the defect or crack based on the accurate measurement of magnetic field immediately at the surface of the structure.

One area of technology that suggests a solution to the nondestructive evaluation inverse problem is neural networks [4]. The knowledge base in the network could be constructed by a machine learning algorithm which would show the waveforms resulting from both good and defective parts. The algorithm would then learn to distinguish between the patterns of good and bad parts. This knowledge would be used by the Neural Network to classify test patterns which it has never seen. If the algorithm has successfully learned the training data, it should then be able to classify new test data accurately [5]. 
Any changes in the knowledge base resulting from changes in test equipment or in the geometry of parts being tested could easily be implemented by rerunning the neural network model, Figure 1.

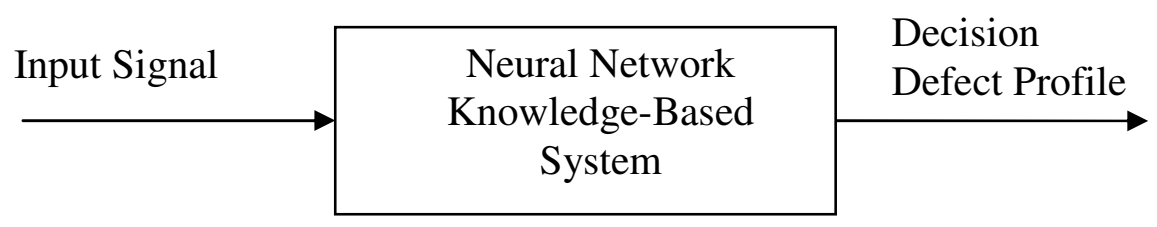

Figure 1 Basic automated system for NDE methods

The advantages of using Neural Networks are numerous: Neural networks are learning machines that can learn any arbitrary function that is mapped between input and output data. They are fast and can be implemented in parallel either in software or in hardware [6].

Different defects may give rise to totally similar signals. That is why it is very important to use the neural network inverse problem to predict the three-dimensional profile of the crack or defect. Using three-dimensional modeling analysis can help in achieving this goal. The user will be able to change the input signal, material, boundary conditions or loads in the interactive visual environment and see the changes in the signatures.

These simulations can be set up on a local PC computer using an interactive visual environment and perform calculations for these simulations by using a supercomputer elsewhere. As a result, this will give the capability to provide an enhanced assessment tool for judgment and decision-making. 


\subsection{Aircraft Aging}

Aging of metallic structures is mostly associated with fatigue cracking, debonding and corrosion. Structural fatigue cracking is a direct result of aircraft use; i.e. load or stress cycles that will eventually occur in all aircraft.

Corrosion results from the exposure of susceptible materials to various corrosive environments, e.g. humid air, salt water, sump tank water and latrine leakage, and to inadequate or the deterioration of corrosion protection systems. Detection and characterization of fatigue cracking and corrosion at the initiation stages, while hidden under concealed areas, make it a great challenge for any inspection technology [7].

From observation, fatigue cracks generally grow on surfaces normal to the direction of the maximum principal stress. So that, once a crack has initiated, it tends to grow at the expense of other putative cracks and soon becomes the dominate crack controlling the residual strength, or the residual fatigue life of the structure.

Under conditions of high cyclic strain, cracks initiate readily and the initiation occurs preferentially at a free surface, resulting in a fine array of small surface breaking cracks. Fatigue cracks have been found to initiate and grow in the radial direction in ageing mechanical structures with components of annular shape [8][9]. For example, fatigue cracks generally originate at the base of the rivet counter sink and progress radially away from the rivet center [10].

It is important to detect these small cracks as early as possible. If the detection technique is not able to detect these small cracks then the separate small cracks can eventually link up forming a relatively large crack. 
On the other hand, corrosion is a relatively slow material degradation process to which metallic aircraft structures are subjected during service. It is a general term that describes the oxidative degradation of metals caused by a local galvanic cell between the base metal (acting as anodic site), at sites of defective protective coating, having the passive sites sustaining cathodic reaction. The corrosion process converts the metal into its oxide or a hydroxide forms resulting in deterioration of its mechanical and electrical properties [11].

Corrosion in aluminum alloys and plated steel surfaces can often be recognized by dulling or pitting of the area, and sometimes by white or red powdery deposits. It may also be the origin of delamination, cracking, metal thinning, fretting, etc. Corrosion can appear in many forms, depending on the type of metal, how it is processed, the surrounding structures and service conditions.

As a result, fatigue cracking and corrosion are a costly source of damage to aircraft and it carries the risk of loss of life as well as hardware in the case of catastrophic failure. As an example, one of the most widely publicized accidents attributed to multiple-site damage was the 1988 incident involving an Aloha Airlines 737 while in flight [12]. The aircraft lost part of its upper fuselage due to multiple site damage resulting from corrosion and disbanding involving multiple rivets with cracks that were not visible through normal maintenance inspections.

This is why the nondestructive evaluation technique should be able to detect small fatigue cracks buried inside structures as well as the longer cracks that extend beyond the edge of the rivet [12]. 


\subsection{Nondestructive Evaluation Methods}

In order to maintain an aircraft defect free and ensure a high degree of quality \& reliability, usually one of the following NDT methods are applied:

Liquid penetrate, Magnetic particle, Ultrasonic, Radiography, Acoustic Emission, Thermography, Visual/Optical, Sonic/Resonance, and Electromagnetic Methods, which will be discussed fully in Chapters Two and Four.

\subsubsection{Liquid Penetrant}

Liquid penetrant testing is one of the oldest of the modern nondestructive testing methods and is widely used in aircraft maintenance. Liquid penetrant testing can be defined as a physical \& chemical nondestructive procedure designed to detect $\&$ expose surface connected discontinuities in 'nonporous' engineering materials [13].

The basic principle of penetrant testing is to increase the visible contrast between a discontinuity \& its background. This can be achieved by treating the area with a liquid of high mobility \& penetrating power, and then encouraging the liquid to emerge from the developer, to reveal the flaw pattern under white light or under ultraviolet light.

The objective of liquid penetrant testing is to rapidly \& economically provide visual evidence of cracks, porosity, laps, or surface discontinuities with a high degree of reliability. This Method can be used in the detection of surface defects or structural damage in all materials of an aircraft. Fluorescent penetrants are used in critical areas for more sensitive evaluation.

\subsubsection{Magnetic Particle}

Magnetic particle testing is a method used for nondestructive testing for surface breaking and some subsurface defects in ferromagnetic materials. This testing method is 
based on the principle that magnetic flux in a magnetized object is locally distorted by the presence of a defect. This distortion causes some of the magnetic field to exit and reenter the test object at the defect. This phenomenon is capable of attracting finely divided particles of magnetic materials that in turn form an indication of the defect location.

The fluorescent particle inspection method is evaluated by black light (Black light consists of a 100 watt mercury vapor projection spot lamp equipped with a filter to transmit a wave length between 3200 to 3800 Angstrom and absorb substantially all visible white light) [13].

This method is simple in principle and easily portable. However, this method requires a special treatment for surface before applying the particles to the surface, it involves a direct contact with the surface and there maybe a chemical interaction between the structure material and the particles.

\subsubsection{Ultrasonic}

Sound with a frequency above the limit of audibility is called ultrasonic. It ranges with a frequency of $0.2 \mathrm{MHz}$ to $800 \mathrm{MHz}$. [12].Ultrasonic techniques are often applied to the detection of disbands and thickness gauging in layered materials. This is easily performed in relatively thick layers where the echoes from the front and back surfaces of the layers are resolved. However in thin aluminum sheets, such as found in most aircraft fuselages, the echoes are difficult to separate in time [14][15]. For this case, the ultrasonic response of the layer is more difficult to interpret.

The resolution of ultrasonic techniques depends on the signal frequency and the size of the transducer; high frequency and small transducer size result in fine resolution, but the depth of penetration is reduced significantly [12][16]. 


\subsubsection{Radiographic Technique}

Radiographic inspection is different from the other techniques in its ability to perform full volume inspections. Its major disadvantage is safe application, which limits access to the aircraft during the inspection. One of the possible applications for this system is the detection of corrosion.

The change in $\mathrm{x}$-ray intensity after the $\mathrm{x}$-rays have passed through a structure is a function of the amount of material along the path taken by the x-rays [12]. Since this functional dependence is well known, it is possible to determine from the change in intensity, the amount of material loss along the path.

\subsubsection{Acoustic Emission Technique}

In Acoustic Emission nondestructive methods, sensors monitor structures for characteristic acoustic signals that indicate the onset or progression of damage or deterioration.

This method is passive in that structures under inspection are not excited with any externally produced energy such as heat, electromagnetic waves, or ultrasonic waves. Crack growth in structures often causes the instantaneous frequency of an acoustic emission signal to increase as a function of time [12][17].

\subsubsection{Thermography Technique}

Thermography is a relatively new technique; it involves heating the surface of a structure and measuring surface temperatures as a function of time. For aircraft lap joint inspections, flash lamps are used to apply heat to the exterior surface of the aircraft and the temperature of the exterior surface is measured with an infrared camera. In a 
relatively short period of time, heating the surface creates temperature differentials which indicate the presence of disbands or corrosion [12].

The main advantage of this method is that it does not require physical contact or coupling between the inspection system and the aircraft. The disadvantage is that the surface of the structure needs to be coated with a high emissivity material before the test which may not be practical in many cases.

\subsubsection{Visual/Optical}

Visual inspection is probably the most widely used of all the nondestructive tests. It is simple, easy to apply, quickly carried out and usually low in cost. The basic principle used in visual inspection is to illuminate the test specimen with light and examine the specimen with the eye. This method is mainly used to magnify defects which can not be detected by the unaided eye, to assist in the inspection of defects and to permit visual checks of areas not accessible to the unaided eye.

\subsubsection{Sonic /Resonance}

Sonic and resonance testing methods are used primarily for the detection of separations between layers of laminated structures. Sonic and Resonance testing is effective for the detection of a crushed core or debonds in adhesive bonded honeycomb, impact damage and delamitations in composite structures and exfoliation corrosion. The tap test method has demonstrated the ability to detect cracks, corrosion, impact damage and debonding. The sonic testing instrument operates in the audio or near audio frequency range.

Resonance testing instruments may operate in either, or both, the sonic or ultrasonic frequency range. Different methods of transmitting and receiving energy have 
been developed. Basically, each technique introduces a pressure wave into the specimen and then detects the resonant, transmitted or reflected wave [7].

\subsection{Inverse Problem in Nondestructive Evaluation Test}

The primary objective of nondestructive evaluation is to characterize the flaw based on the measurement signal. This can be accomplished using signal classification algorithms or alternately via algorithms for estimating flaw parameters given the measured signal. The problem of flaw characterization can be represented using a systems approach to nondestructive evaluation.

A typical nondestructive evaluation system can be represented by the linear model, Figure 2, where $x(t)$ is the excitation source, $y(t)$ is the probe measurement and $F(\omega)$ is the transfer function of the field/flaw interaction [6][18][19].

Three classes of problems may be defined using this approach:

1- Given input $x(t)$ and system $F(\omega)$, determine the output $y(t)$.

2- Given input $x(t)$ and output $y(t)$, determine $F(\omega)$.

3- Given system $F(\omega)$ and the output $y(t)$, determine $x(t)$.

$\longrightarrow \begin{aligned} & \text { N. N. Knowledge-Based } \\ & \text { System } F(\omega)\end{aligned}$

Figure 2: Systems approach to NDE [20] 
The first case presents the forward problem while the second and third cases are related to inverse problems. In NDE, the forward problem involves estimating the measurement signal due to a flaw and applied input energy whereas inverse problems involve the estimation of defect parameters using information contained in the measurement signal. Defect parameters can range from simple estimates of equivalent length, width and depth to a full three-dimensional profile.

An inverse problem is said to be well-posed if the solution satisfies three properties:

1- Existence

2- Uniqueness

3- Continuity, the solution depends continuously on the input.

The forward problem in general is well-posed and is solved analytically or by means of numerical modeling. In contrast, inverse problems in general are ill-posed, lacking both uniqueness and continuous dependence of the measured signals. This has resulted in the development of a variety of solution techniques ranging from simple calibration procedures to other direct and iterative approaches [6][21][19].

These solution techniques can be divided into two broad classes:

The first class attempts to solve the inverse problem by using signal processing techniques. These techniques typically range from simple calibration methods to the more recent procedures based on neural networks. Calibration curves are obtained by first generating a set of signals either experimentally or numerically for a range of defect parameter values. 
A family of calibration curves is obtained by plotting signal features such as peak values against defect parameters such as defect depth while holding other parameters constant. These curves are then used to predict defect parameters for a given signal. Direct solutions involve mapping the measured signal directly to the flaw parameters. An example of this approach is the use of neural networks to map the measurement to the required defect profile. In this case, the problem is formulated as a function approximation problem and the underlying function mapping of the input signal to the output (profile) is learned by a neural network.

The second class can be direct or iterative. Iterative approaches typically employ a forward model that simulates the underlying physical process. The physical process in NDE is usually represented by means of differential or integral equations, and the corresponding forward model is typically a numerical model such as a finite element model.

The algorithm starts with an initial estimate of the defect parameters and solves the corresponding forward problem to determine the signal. The error between the measured and predicted signals is minimized iteratively by updating the defect profile. When the error is below a pre-set threshold, the defect parameters represent the desired solution.

\subsection{Research Objectives}

The main objective of this research is to develop a new electromagnetic way of performing a nondestructive evaluation (NDE) technique for non-ferromagnetic aircraft components and structures that will allow solving the inverse problem by using a neural network approach and visualizing the signatures of the defects or cracks in three 
dimensions. This will give the user better understanding about the fields near the defects or cracks on the surface of the structure or inside it, which is not available in current nondestructive evaluation methods. This capability will provide a very important tool for engineers to study and perform inspections of aircraft structures.

To achieve the above objectives, a library of different magnetic models is required. The modeling needed to develop this library will involve a reconstruction of the magnetic field distribution generated by various possible defects of different dimensions and orientations, within the component. This library of data will be used in training the Neural Network.

The magnetic field can be generated by electrical current passing through the component or by response to an external magnetic field applied to the component. The goal is to detect the crack and find its location and its profile.

At the end, the proposed system will be able to integrate results from computer modeling and neural network tools and then test the results with real measurement data. The final product will be able to solve the electromagnetic nondestructive evaluation problem.

\subsection{Organization of This Dissertation}

This dissertation is organized as follows: Chapter Two presents a brief introduction to the electromagnetism theory. This section contains a discussion of different electromagnetic nondestructive evaluation (NDE) methods that are used to detect cracks and defects in mechanical structures. This section establishes the theoretical foundation for the magnetic flux leakage method (MFL) applied to non-ferromagnetic structures. 
Chapter Three presents the research methodology and approach in details. This section of the dissertation outlines the mechanical and electromagnetic analyses that were carried out to establish the relation between the mechanical stressistrain fields and the electrical current and magnetic field distribution in the structure. It also contains a subsection about solving the nondestructive evaluation inverse problem by using a neural network model. Another subsection discusses the limitations of this analysis.

Chapter Four presents the proposed approach for applying the magnetic flux leakage method for non-ferromagnetic structures, aluminum plates, which contain a defect or crack. Mechanical and electromagnetic analyses are presented using finite element modeling and analytical solutions.

The analytical solution is provided to calculate the magnetic field produced by the non-ferromagnetic structure and the crack. A detailed discussion is provided to study the effect of any change in the crack profile on the magnetic field above the structure.

Chapter Five presents a brief introduction to the neural network analysis and the proposed approach to solve the inverse problems, namely, a simple neural network based iterative inversion algorithm, Back-Propagation.

The necessary equations along with a complete description of the algorithm are presented. Results of applying this algorithm are also presented in the corresponding subsections.

Chapter Six discusses the experimental verification work for different plates that contain different defect sizes. Results from both modeling analysis and experimental analysis are compared and a discussion of error sources is provided. 
Chapter Seven summarizes this research results and conclusions. It also summarizes the various approaches and presents ideas as recommendations for future work.

Finally, a brief description of the tools and computer software, ABAQUS and MATLAB, used in this research is provided in the Appendices. 


\section{Electromagnetic Nondestructive Evaluation Method}

Electromagnetic testing has its origins when Oersted (1824) discovered that current passing through a coil created a magnetic field capable of shifting a compass needle. Seven years later, Faraday discovered the opposite; that a moving magnetic field would induce a voltage in an electrical conductor.

In 1879 Hugh recorded changes in the properties of a coil when placed in contact with metals of different conductivity and permeability. But it took to the time of the Second World War that these effects were put to practical use for the testing of materials [18]. Much work was done in the 1950's and the 60's, particularly in the aircraft and nuclear industries. Electromagnetic testing methods are now an accurate and widely used inspection Technique.

\subsection{Concepts of Electromagnetism}

Magnetic fields are produced by electric currents, which can be macroscopic currents in wires, or microscopic currents associated with electrons in atomic orbits, Figure 3. The magnetic field $\mathrm{B}$ is defined in terms of force on a moving charge in the Lorentz force law. The interaction of a magnetic field with a charge leads to many practical applications. Magnetic field sources are essentially dipolar in nature, having a north and south magnetic pole. The SI unit for magnetic field is the Tesla, which can be seen from the magnetic part of the Lorentz force law $F_{\text {magnetic }}=q v B$ to be composed of (Newton $\mathrm{x}$ second)/ (Coulomb $\mathrm{x}$ meter). A smaller magnetic field unit is the Gauss (1 Tesla $=10,000$ Gauss $)$ 

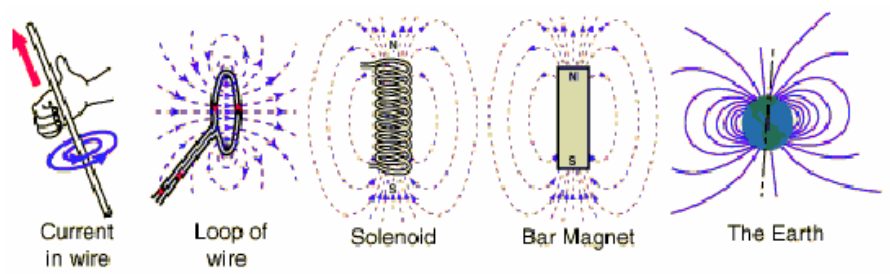

Figure 3 Magnet sources [23]

The relationship between the magnetic field contribution and its source current element is called the Biot-Savart law, Figure 4. The Biot-Savart Law relates magnetic fields to the currents which are their sources. In a similar manner, Coulomb's law relates electric fields to the point charges which are their sources. Finding the magnetic field resulting from a current distribution involves the vector product, and is inherently a calculus problem when the distance from the current to the field point is continuously changing [23][24][25].
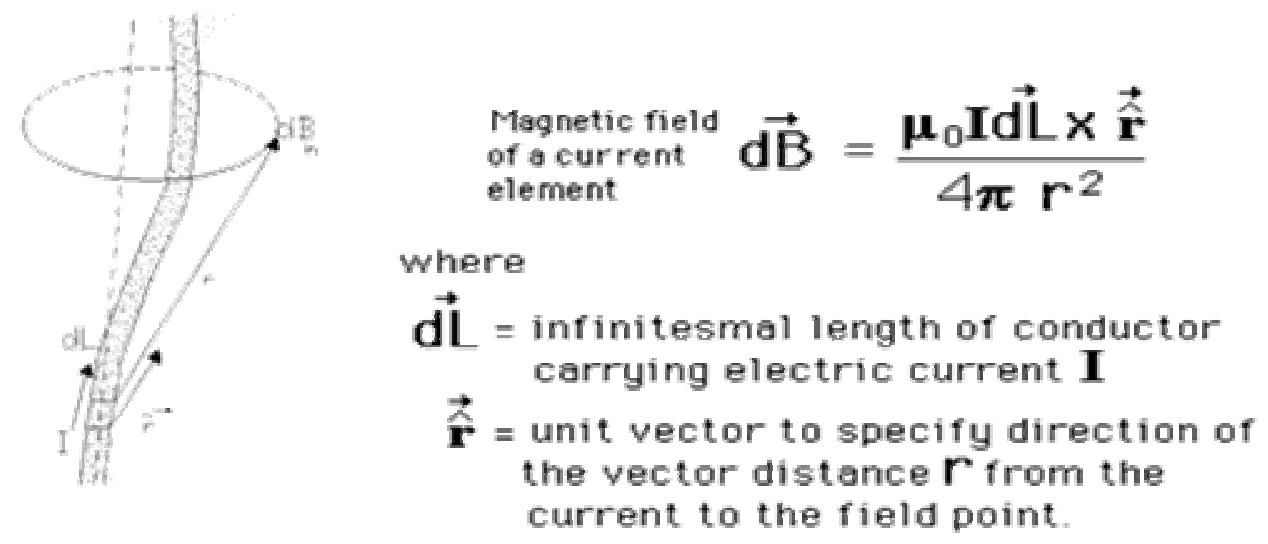

Figure 4 The Biot-Savart Law [26]

Each infinitesimal current element makes a contribution to the magnetic field at point $P$ which is perpendicular to the current element, and perpendicular to the radius 
vector from the current element to the field point $P . d \vec{B}$ is the magnetic field contribution at $P$ from the current element $I d \vec{B}$ [23].

The direction of the magnetic field contribution follows the right hand rule illustrated for a straight wire. This direction arises from the vector product nature of the dependence upon electric current.

Magnetic flux is the product of the average magnetic field times the perpendicular area that it penetrates, Figure 5. It is a quantity of convenience in the statement of Faraday's Law and in the discussion of objects like transformers and solenoids [27][28][29].

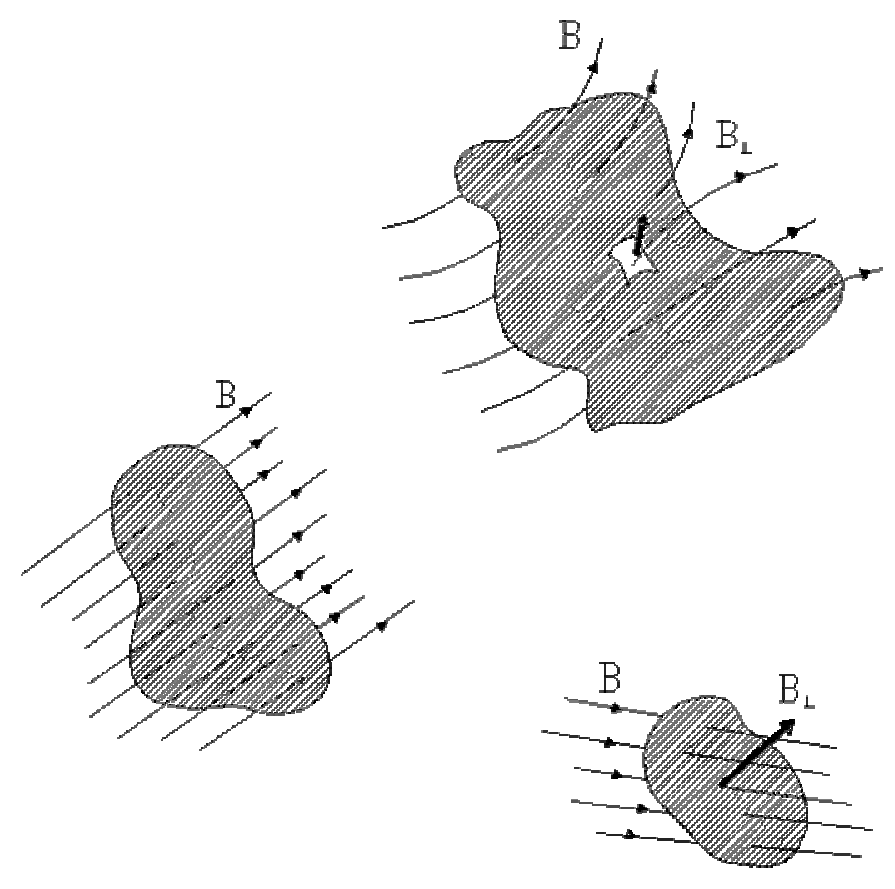

Figure 5 Magnetic Flux Illustrations [23] 
The magnetic field in space around an electric current is proportional to the electric current which serves as its source, just as the electric field in space is proportional to the charge which serves as its source. Ampere's Law states that for any closed loop path, the sum of the length element times the magnetic field in the direction of the length element is equal to the permeability times the electric current enclosed in the loop.

The magnetic fields generated by currents and calculated from Ampere's Law or the Biot-Savart Law is characterized by the magnetic field B measured in unites of Tesla. But when the generated fields pass through magnetic materials which themselves contribute internal magnetic fields, ambiguities can arise about what part of the field comes from the external currents and what comes from the material itself. It has been common practice to define another magnetic field quantity, usually called the "magnetic field strength" designated by H [27]. It can be defined by the relationship

$$
H=B_{0} / \mu_{0}=B / \mu_{0}-M
$$

Eq. 1

The relationship for B can be written in the equivalent form of:

$$
B=\mu_{0}(H+M) .
$$

$H$ and $M$ will have the same units, amperes/meter. To further distinguish $B$ from $H, B$ is sometimes called the magnetic flux density or the magnetic induction. The quantity $M$ in these relationships is called the magnetization of the material.

Another commonly used form for the relationship between $\mathrm{B}$ and $\mathrm{H}$ is:

$$
B=\mu_{\mathrm{m}} H,
$$

where

$$
\mu=\mu_{m}=K_{m} \mu_{0},
$$


$\mu_{0}$ being the magnetic permeability of space and $\mathrm{K}_{\mathrm{m}}$ the relative permeability of the material. Relative permeability is a quantity which measures the ratio of the internal magnetization to the applied magnetic field. If the material does not respond to the magnetic field by magnetizing, then the field in the material will be just the applied field and the relative permeability is $\mathrm{K}_{\mathrm{m}}=1$. A positive relative permeability greater than 1 implies that the material magnetizes in response to the applied magnetic field.

The quantity $\chi_{\mathrm{m}}$ is called magnetic susceptibility, and it is just the permeability minus 1 . The magnetic susceptibility is then zero if the material does not respond with any magnetization. So both quantities give the same information, and both are dimensionless quantities. Magnetic properties of some common materials are shown in Table 1 [23][24][25].

For ordinary solids and liquids at room temperature, the relative permeability $\mathrm{K}_{\mathrm{m}}$ is typically in the range 1.00001 to 1.003 . This weak magnetic character of common materials is recognized by saying "they are not magnetic", which recognizes their great contrast to the magnetic response of ferromagnetic materials. More precisely, they are either paramagnetic or diamagnetic, but that represents a very small magnetic response compared to ferromagnets.

In the equations describing electric and magnetic fields and their propagation, three constants are normally used. One is the speed of light c, and the other two are the electric permittivity of free space $\varepsilon_{\mathrm{o}}$ and the magnetic permeability of free space, $\mu_{\mathrm{o}}$. 


\begin{tabular}{|l|l|}
\hline Material & $\begin{array}{l}\chi_{\mathrm{m}}=\mathrm{K}_{\mathrm{m}}-1 \\
\left(\mathrm{x} 10^{-5}\right)\end{array}$ \\
\hline Steel, 0.9\% C & \multicolumn{1}{|c|}{50} \\
\hline Iron aluminum & 66 \\
\hline Uranium & 40 \\
\hline Platinum & 26 \\
\hline Aluminum & 2.2 \\
\hline Sodium & 0.72 \\
\hline Oxygen gas & 0.19 \\
\hline
\end{tabular}

Table 1 Magnetic properties of some common materials [24][25]

The magnetic permeability of free space is taken to have the exact value $\mu_{0}=4 \pi \times 10^{-7} \mathrm{~N} / \mathrm{A}^{2}$ and then the electric permittivity takes the value given by the relationship

$$
c=\frac{1}{\sqrt{\mu_{o} \varepsilon_{o}}},
$$

Eq. 5

Where, $\mathrm{c}=2.99792458 \times 10^{8} \mathrm{~m} / \mathrm{s}$.

This gives a value

$$
\varepsilon_{o}=8.854187817 \times 10^{-12} \mathrm{~F} / \mathrm{m} \approx 8.85 \times 10^{-12} \mathrm{~F} / \mathrm{m}
$$

Eq. 6

In the presence of magnetic media, the effective constants will have different values [23][24]. 


\subsection{Electromagnetic Nondestructive Evaluation Methods}

Researchers classify three methods under electromagnetic inspection techniques: Electrical Potential, Eddy Current, and Magnetic Flux methods.

\subsubsection{Electrical Potential Drop Method}

It is known that the passage of an electrical current through a metal body will be affected by the presence of a crack or defect. This deflection of the current is detected by measuring the potential difference between various parts of the body especially in the region of the crack [30].

The potential drop technique uses four contacting pins that are arranged in the same line and used for a current supply and measurement of potential difference. The changes in the potential difference across the crack can often be interpreted in terms of crack depth using a calibration block containing cracks of known depth [16][31].

\subsubsection{Eddy Current Method}

Two common methods exist for eddy current testing, a single coil and a dual coil method. Both rely on the principle that as coil is excited with an AC voltage, flux lines emanate from one face of the coil, arch around in the space surrounding the coil, and enter the opposite face of the coil. The total amount of flux generated is directly proportional to the amount of current flowing through the coil [32].

The flux lines will close on themselves without crossing any other lines, but if any flux line impinges upon a conducting object, a small whirlpool-like eddy current will be created at the surface of the object under consideration. These eddy currents, in turn produce a magnetic field, which opposes that field which was generated by the driving coil. 
The properties of eddy currents are heavily influenced by the electric conductivity and magnetic permeability of the media in which the currents flow; the greater the conductivity of a material the greater the flow of eddy currents on the surface, some factors that can affect the conductivity are grade (material composition), heat treatment, work hardening, etc. On the other hand, permeability is described as the ease with which a material can be magnetized. Permeability will vary greatly due to localized stresses, fatigue cracking, corrosion and other kinds of defects [12][32][30].

Therefore, there will be a localized change in induced eddy currents while inspecting a defective structure. The detection of defects as small as $0.8 \mathrm{~mm}$ has been reported, which correspond to cracks that have been observed beneath typical fastener heads [12].

Selection of the operating frequency is the primary eddy current test parameter. The high frequency excitation is usually used to improve the sensitivity of the response signals from cracks and defects on the material surface in the sensor side. Inversely, it means that the sensitivity of the response signals from the opposite surface of the conductor is reduced due to the skin effect. So, eddy current probes rely on a decrease in the effective electrical conductivity in the direction normal to the crack. This decrease is

restricted to surface layer of depth approximately equal to the average depth of the surface crack [12][15].

\subsubsection{Magnetic Flux Leakage Method}

Magnetic techniques have been shown to be sensitive to microstructural changes induced by cyclic stress. The discontinuity resulting from a crack also produces a disturbance to the distribution of magnetic field in the material, and the magnitude of the 
disturbance is determined by the size and shape of the crack. Therefore, it should be possible to evaluate the crack area by magnetic measurements under localized magnetization [33].

In this work, a new Electromagnetic NDE method is proposed by measuring magnetic flux leakage on the top of a conducting plate. The method is a magnetic flux leakage method applied to nonmagnetic material like Aluminum. The magnetic field distribution on the top of a conducting plate can be calculated by considering the current density distribution in the plate. [2]

The relationship between the magnetic field contribution and its source current element is called the Biot-Savart law. The Biot-Savart Law relates magnetic fields to the currents which are their sources.

Each infinitesimal current element makes a contribution to the magnetic field at point $P$ which is perpendicular to the current element, and perpendicular to the radius vector from the current element to the field point $P . d \vec{B}$ is the magnetic field contribution at $P$ from the current element $I d \vec{L}$.

Sensors are used to detect any leakage flux caused by the presence of the crack or defect. These sensors could be coil sensors, Hall Effect sensors, flux gate magnetometers, SQUID magnetometers and nuclear magnetic resonance magnetometers. All of these magnetometers work well for detecting the existence of a crack, but cannot be used to visualize them in three dimensions and it is difficult to obtain sub-millimeter spatial resolution. 


\subsection{Magnetic Flux Leakage Theory}

The magnetic field produced by a current flowing in a plate can be calculated at any location above the plate. The basic example is a steady flow of electric current in a thin rectangular plate of width $b$, length $d$, and thickness $t$, the plate is homogeneous and isotropic with conductivity $\sigma$. For initial analysis, it is assumed that the right and left ends of the plate are maintained at potential $+V_{\mathrm{o}}$ and $-V_{\mathrm{o}}$, respectively, Figure 6 . To study the magnetic field produced by the current at a height $z_{\mathrm{o}}$ above the $x^{\prime} y^{\prime}$ plane, we need first to calculate the current density distribution within the plate. The geometry of the problem as well as the boundary conditions are shown, where the primed coordinates refer to the electric current while the unprimed ones refer to the magnetic field.

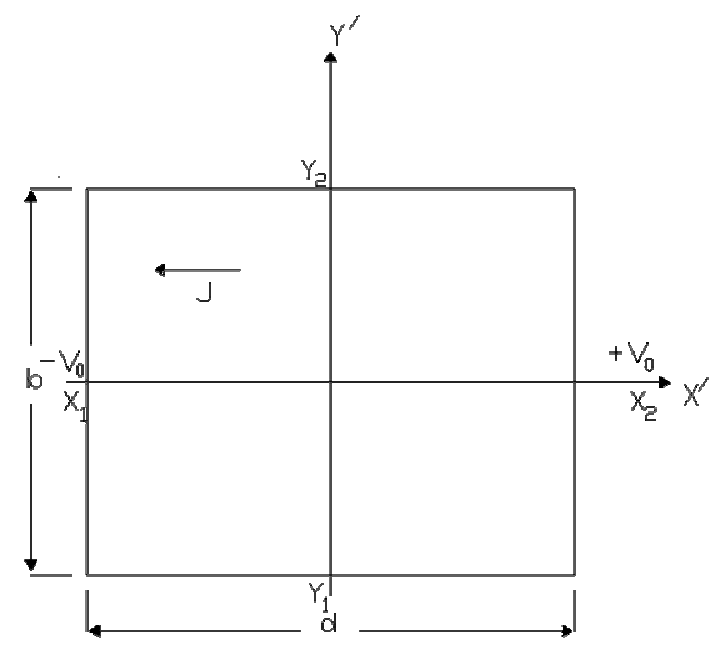

\section{Figure 6 Plate under consideration}

For a thin homogeneous and isotropic plate without a crack, the potential distribution is $\phi\left(x^{\prime}, y^{\prime}\right)$ that satisfies Laplace's equation in two dimensions is [24][25]:

$\nabla^{2} \phi\left(\mathrm{x}^{\prime}, \mathrm{y}^{\prime}\right)=0$

Eq. 7 
with boundary conditions as

$$
\phi\left(d / 2^{\prime} y^{\prime}\right)=+V_{\mathrm{o}} \quad \text { and } \quad \phi\left(-d / 2^{\prime} y^{\prime}\right)=-V_{\mathrm{o}} \quad \text { Eq. } 8
$$

The current density distribution $\vec{J}\left(x^{\prime}, y^{\prime}\right)$ is expected to be uniform with a direction anti-parallel to the $x$-axis and the corresponding potential $\phi$ depends only on $x$. Thus Eq. 7 reduces to

$$
\partial^{2} \phi\left(x^{\prime}\right) / \partial x^{2}=0
$$

And the potential is obtained as

$$
\phi\left(x^{\prime}\right)=K x^{\prime}+L
$$

The boundary condition given by Eq. 8 can be applied to determine the constants $K$ and $L$ to obtain

$$
\phi\left(x^{\prime}\right)=2 V_{o} x^{\prime} / d
$$

The current density is

$$
\overrightarrow{\mathrm{J}}\left(\mathrm{x}^{\prime}, \mathrm{y}^{\prime}\right)=\overrightarrow{\mathrm{J}}=-\sigma \frac{\partial \phi\left(x^{\prime}\right)}{\partial x^{\prime}} i=\frac{2 V_{0} \sigma}{d} i
$$

Which is a constant in the $x$-direction as given by the unit vector $i$. [25]

Now the magnetic field $\vec{B}(\vec{r})$ at any point external to a volume v' containing a distribution of current $\overrightarrow{J(} \vec{r})$ is given by the law of Biot-Savart as [26][27][45]

$$
\vec{B}(\vec{r})=\frac{\mu_{0}}{4 \pi} \int_{v^{\prime}} \frac{J\left(\overrightarrow{r^{\prime}}\right) \times\left(\vec{r}-\overrightarrow{r^{\prime}}\right)}{\left|\vec{r}-\vec{r}^{\prime}\right|^{3}} d x^{\prime} d y^{\prime} d z^{\prime}
$$

where $\mu_{0}=4 \pi \times 10^{-7} T . m / A$ is the magnetic permeability of the free space. For a twodimensional current distribution flowing in a thin plate located in the $x, y^{\prime}$ plane, the 
integral over $d z^{\prime}$ reduces to a multiplicative factor $t$ for the thickness in the $z^{\prime}$-direction. In this case Eq. 13 becomes an integral over the surface $S$,

$$
\vec{B}(\vec{r})=\frac{\mu_{0} t}{4 \pi} \int_{s^{\prime}} \frac{J\left(\overrightarrow{r^{\prime}}\right) \times\left(\vec{r}-\overrightarrow{r^{\prime}}\right)}{\left|\vec{r}-\overrightarrow{r^{\prime}}\right|^{3}} d x^{\prime} d y^{\prime}
$$

The magnetic field $\vec{B}(\vec{r})$ has three components $B_{x}, B_{y}$, and $B_{z}$ that can be determined by expanding the cross-product in Eq. 14 [27]. The magnetic field component $B_{z}$ is the component that is of interest in this study, since this is the component that the present Hall sensor can measure.

$$
B_{z}(x, y, z)=\frac{\eta_{0} t}{4 \pi} \int_{s^{\prime}} \frac{J_{x^{\prime}}\left(x^{\prime}, y^{\prime}\right)\left(y-y^{\prime}\right)-J_{y^{\prime}}\left(x^{\prime}, y^{\prime}\right)\left(x-x^{\prime}\right)}{\left[\left(x-x^{\prime}\right)^{2}+\left(y-y^{\prime}\right)^{2}+\left(z-z^{\prime}\right)^{2}\right]^{3 / 2}} d x^{\prime} d y^{\prime} .
$$

Since the $y^{\prime}$ component of the current density $J_{y^{\prime}}\left(x^{\prime}, y^{\prime}\right)$ is zero, the $x^{\prime}$-component of the current density $J_{x^{\prime}}\left(x^{\prime}, y^{\prime}\right)$ is the constant $J_{0}$ in Eq. 12 , and by taking $z^{\prime}=0$, then Eq. 15 reduces to

$$
B_{z}(x, y, z)=\frac{\eta_{0} t}{4 \pi} J_{0} \int_{s^{\prime}} \frac{\left(y-y^{\prime}\right)}{\left[\left(x-x^{\prime}\right)^{2}+\left(y-y^{\prime}\right)^{2}+(z)^{2}\right]^{3 / 2}} d x^{\prime} d y^{\prime} .
$$

One more important issue needs to be addressed. If the current is applied and removed at discrete points rather than along the entire edges of the plate, there will be a spreading of current in the vicinity of the connection between the external wires and the plate and this means the current is not uniformly supplied and removed from the plate. The magnetic field $B_{z}$ will differ from that of the uniform current. More details about dealing with this problem will be discussed in Chapter 4. 


\section{Research Approach and Methodology}

This chapter presents the problem definition, research approach and the numerical analysis to be carried out for the purpose of solving the nondestructive evaluation problem.

\subsection{Problem Definition}

A major problem in electromagnetic NDE methods is the ability to predict the defect location given the measured signal. Identification of the exact location of the crack types, size, and shape is another important issue which is not fully addressed by the current Nondestructive Evaluation Techniques [18][37].

Many electromagnetic NDE tests are presently carried out and interpreted manually. In most cases, a technician scans the part being tested and observes the output signal. Based on his level of training and experience in conducting the test, he will make a decision as to whether the structure is good or bad. This experience is related to the features that can be seen in the output signal. Since these features should be consistent for a specific structure and are directly related to the type of the defect, it would seem that an automated system could be constructed to mimic the human experience and help human technicians in performing reliable and efficient testing.

So, in order to reduce the cost and increase testing efficiency and reliability, which will improve the safety of non-ferromagnetic metallic structures and save lives, it is necessary to develop a highly reliable and fast way to perform Nondestructive Evaluation Techniques, which will posses certain unique features. These features include remote detection, environmental compliance, safety and ease of operation, functionality in real-time and on-site, and ease of adaptability to large area testing. 


\subsection{Research Approach}

In this work, a new electromagnetic NDE method is proposed by measuring a magnetic flux leakage on the top of a conducting plate. The method is a magnetic flux leakage method applied to nonmagnetic material like Aluminum. The magnetic field can be generated by an electrical current passing through the plate. By using threedimensional modeling analysis we can study the electrical and mechanical stress fields around the crack. It is important to be able to visualize the stress field near the defect, which will help in the prediction of the evolution of defect/ crack transition and propagation.

At the time of inspection, the computer system should be able to visualize the magnetic field measured by the sensor above the surface of the structure, including magnetic field lines and magnetic field intensity. From this information, the computer will provide the description of the crack profile that would generate such a distribution of magnetic flux. This description of the crack profile is obtained by solving the inverse problem where the computer is generating a description of the defect based on the accurate measurement of the magnetic field immediately at the surface of the structure.

To achieve the above objectives, a library of different magnetic models is required. The modeling needed to develop this library will involve a reconstruction of the magnetic field distribution generated by various possible defects of different dimensions within the component. To develop the library of previously calculated response signatures, a modeling analysis is needed. Different models of magnetic flux were done for specific defects of specific location, size, and shape in the component. The signatures, magnetic flux, from all these defects was modeled, categorized and saved in the library 
for the training of the neural network. Once the neural network was trained, it can be implemented in the test as computer software or as a hardware tool.

This system might preprocess the new raw waveforms to highlight features (preprocessing). Then Neural Network techniques could be used to learn waveforms from both good parts and defective parts to construct a system which can characterize new test results as good or bad. In defective cases, it is important to estimate the size and depth of the defect. It is highly desirable to automate the NDE process, since a large number of inspections are performed. Furthermore, this automated system will provide consistent results, especially with regard to small defects and defects close to the front or back surfaces.

The concept of this methodology can be outlined as shown in the following figure:

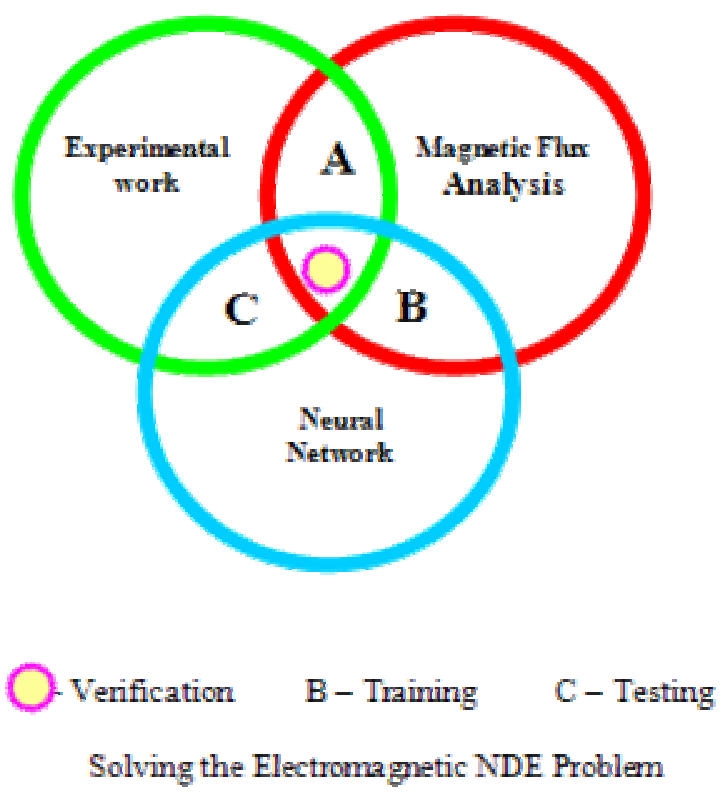

Figure 7 Concept of the research methodology 
Two software packages are used to build the tool that can solve the problem of the NDE; ABAQUS and MaTlab. The final tool will be a design that can be put in practice and easily integrated in the market.

In summary, simple structures are used to model the magnetic flux leakage field above plates with surface cracks. Cracks with different profiles will result in different flux signals. These output signals will serve as an input for the neural network. After the neural network tool is trained, experimental measurements are carried out for verification purposes. The following flow chart will illustrate the approach of solving this NDE method.

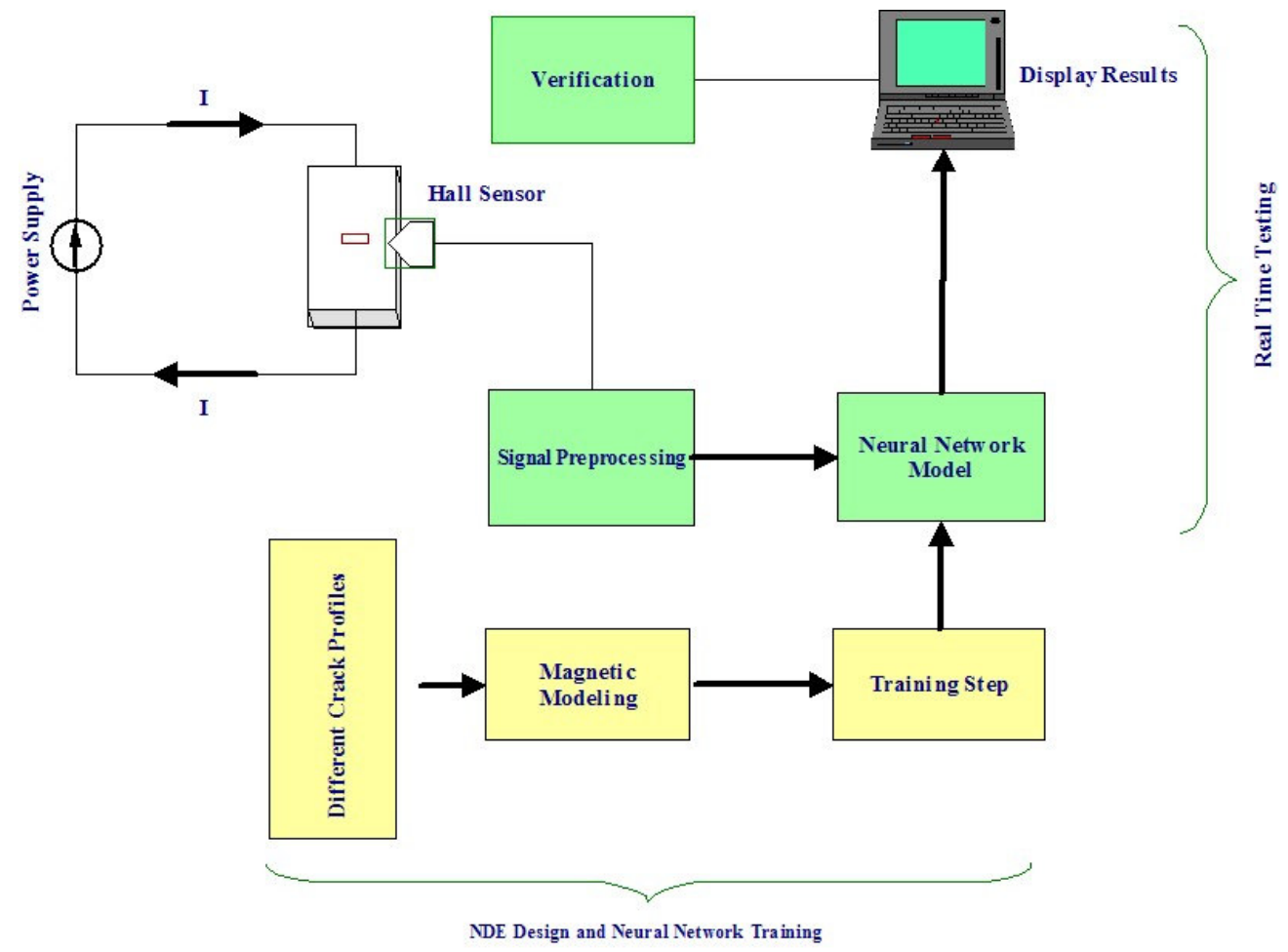

Figure 8 Proposed approach for solving the NDE Problem 


\subsubsection{Mechanical and Electrical Analysis}

In electromagnetic nondestructive evaluation methods, forward problems involve determining the field due to a given permeability distribution. The corresponding inverse problems determine the parameter distribution given the measured fields. An example of the forward problem is where leakage flux due to a flaw in a specimen of complex geometry needs to be determined. Such problems can not be solved by using simple analytical methods and require the use of finite element analysis. Finite element analysis is used extensively in solving both the forward and inverse problems in electromagnetism.

Different structures of Aluminum plates including variable defect parameters will be analyzed. Applying the same load at both ends in opposite directions will result in a change in the stress and strain fields around the crack. Here, a change in the electrical current distribution around the crack can be noticed with the change in the geometry of the crack. Mechanical and electrical analysis for these models was conducted using ABAQUS. Chapter Four provides more details about the mechanical and electrical models.

\subsubsection{Magnetic Field Analysis}

Sending current in a conductor will generate a magnetic flux around the structure. The presence of the defect or crack will change the electrical current distribution which will result in changing the magnetic flux field due to the magnetic flux leakage with the highest value around the crack tip. The change of the magnetic field will result from both the plate edges and the crack. As was mentioned in chapter two, the normal component of the magnetic field, $B_{\mathrm{Z}}$, is the quantity of interest. 
A complete analysis for a wide range of crack profiles to get enough samples was conducted to train the neural network. Three dimensional magnetic field signals and contour plots give detailed information about the relation between the crack profile and the flux signature. Full magnetic analysis is provided in Chapter Four.

\subsubsection{Neural Inverse Problem Approach}

The inverse network predicts the crack profile given the NDE signal. It can be tested with the normal component of Magnetic Flux Leakage (MFL) data generated by three dimensional analyses. Available neural networks in NDE research area address the problem in two dimensions only, in another word, it predicts two dimensions of the crack profile. This study will provide a tool that can predict the crack profile in its three dimensions given that the dimensions are uniform. The Neural Network models can be simulated with the use of the MATLAB neural network toolbox. The number of layers, number of units in each layer, and type of functions that were used in the network was chosen depending upon the application.

In order for the inversion results to be accurate, the inverse network output should be a true inverse of the input signal. The training mode helps to optimize the parameters of the inverse network to achieve this. The performance of a trained network is measured by the errors on the training, validation and test sets. Backpropagation Neural Networks was used in solving the NDE inverse problem whose knowledge base is represented by weighted interconnected arcs [20][21].

Before training, it is often useful to scale the inputs and targets so that they always fall within a specified range [18]. 
A computer program using the neural network functions available in MATLAB Software was written to design the neural network proposed to solve the electromagnetic inverse problem. MATLAB Neural Network Tool Box (MNNTB) contains many functions that can be used in the design of the network depending on the application and number of training models [4][40]. Once the Network/Data Manager is up and running, the network can be created, viewed, and trained. It can simulate and export the final results to the workspace. Similarly, the data can be imported from the workspace for use in the graphical user interface [18].

Chapter Five presents the detailed design of the neural network and discusses the training step in detail; training, validation and test sets are provided and the results are compared to the software modeling.

\subsection{Experimental Testing and Verification}

An experimental procedure was conducted to prove this method concept and to verify the simulation results. Different aluminum plates containing different defect sizes of the same profile were used in the experimental work. In this study, a Hall Effect sensor is used to measure flux density produced by the crack. In order to verify the modeling results, non-ferromagnetic plates were fabricated with dimensions of 6 in $\times 9$ in $\times 0.025$ in $(0.1524 \mathrm{~m} \times 0.2286 \mathrm{~m} \times 0.635 \mathrm{~mm})$ with different defect sizes at the center.

Figure 9 shows the schematic of the experiment needed to confirm the validity of the analysis.

A DC power supply is used to generate a DC current that flows in the plate to produce the electrical field around the crack. The DC power supply available for this 
experiment can generate 5 volts. The resistance of the aluminum plate is very small. Therefore, a $1 \Omega$ resistance is used in series with the plate.

Before conducting the experimental analysis, the sensor needs to be prepared in a way that the magnetic flux readings are representing only the magnetic field resulting from the crack. There is a large field above the plate before applying the current to the plate. It is important to "zero" this field before taking any readings with the sensor.

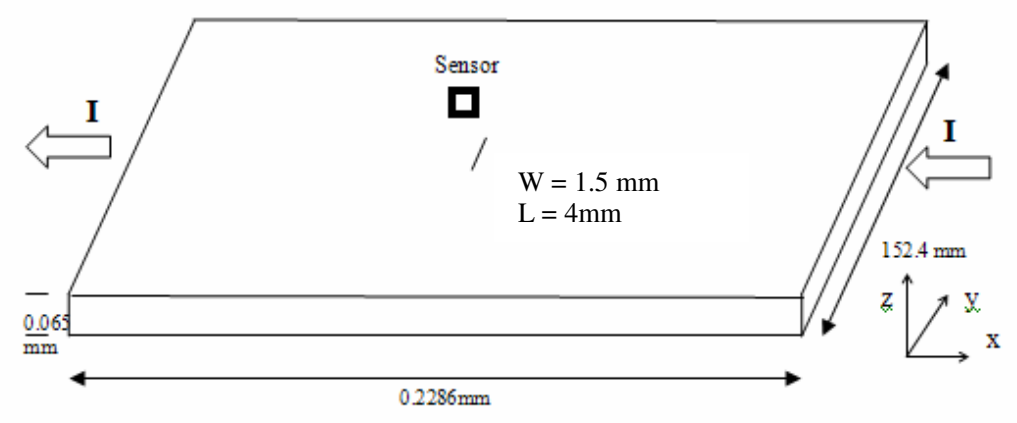

Figure 9 Schematic diagram of the plate under testing

\subsection{Analysis Limitations}

In this study, the proposed method for detecting cracks in aircraft structures can detect any crack in the structure as long as this crack changes the current distribution in the structure. However, the provided analysis in this study addresses only specific structures with limited crack geometries. The structures under investigation are aluminum plates with surface cracks at the center of the plate The crack has uniform dimensions where its length is always normal to the DC current direction as shown in Figure 9.

As discussed in the previous section, the neural network analysis is carried out to predict the crack profile. The neural network is trained by using magnetic field samples 
generated by the simulation analysis using the MATLAB ${ }^{\circledR}$ Software and based on an analytical solution for calculating the magnetic field above a plate that contains a crack with a specific geometry as discussed before. The neural network tool provided in this study can only predict crack profiles identical in shape to those discussed in the magnetic field analysis section. However, this neural network tool has the capability to predict more general crack profiles providing that the training samples are related to these profiles. 


\section{Mechanical and Electromagnetic Analysis}

This chapter presents the mechanical and electromagnetic analysis used in solving the nondestructive evaluation inverse problem. Discussion of the analysis results is provided in the following sections.

\subsection{Mechanical and Electrical Analysis}

The magnetic field is generated by a DC electrical current passing through a conducting plate. Applying mechanical loads on this plate will result in a change in the stress and strain fields around the crack. Here, a change in the electrical current distribution around the crack is noticed with the change in the geometry of the crack. Different structures of Aluminum plates including variable defect parameters are analyzed in finite element softwares. Mechanical and electrical analysis for these models was conducted by using ABAQUS 6.3 software.

The electrical analysis is carried out on the plate shown in Figure 10, and by using

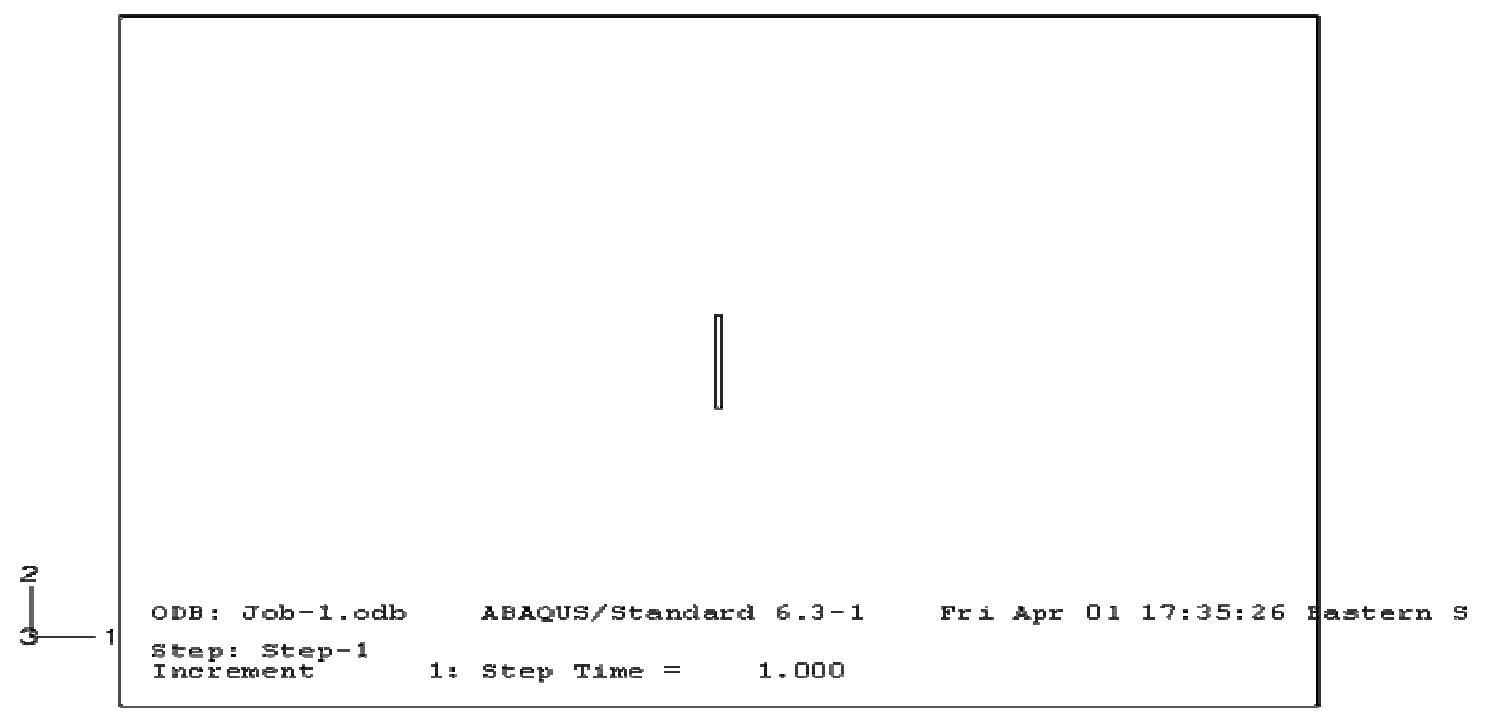

Figure 10 Structure modeling in ABAQUS 
the symmetry in the plate to carry out the analysis on the model as shown in Figure 11.

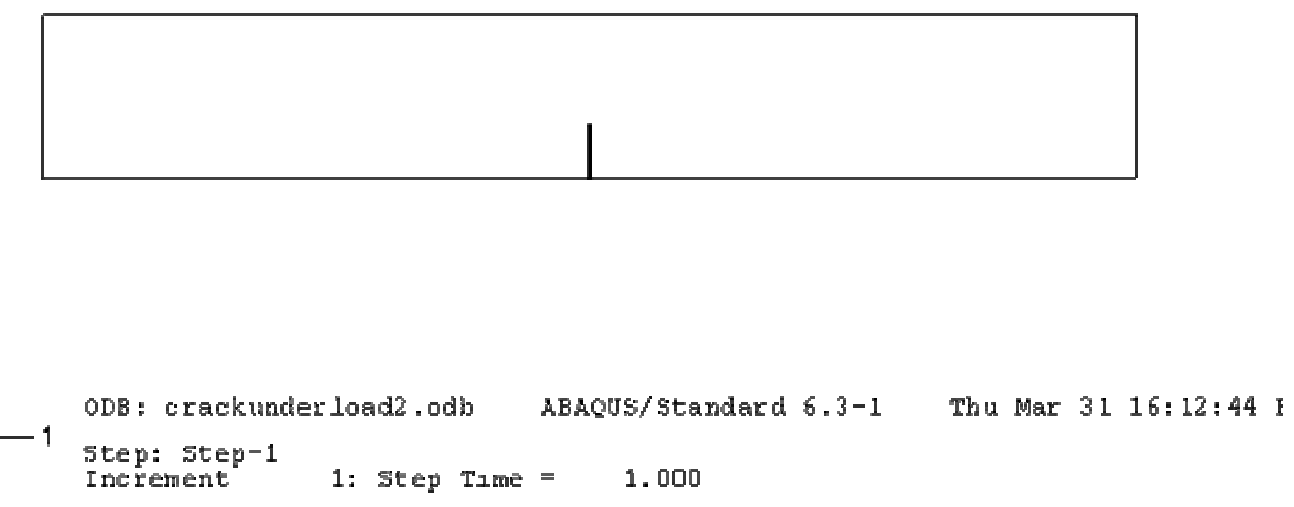

Figure 11 Structure modeling in ABAQUS using symmetry

The plate was assigned the properties of Aluminum metal where young's modulus is taken as $69.1 \mathrm{GPa}$, Poisson's ratio as 0.33 , and the density of the material is defined in the property module of the software as $2700 \mathrm{~kg} / \mathrm{m}^{3}$. The boundary conditions are defined in the LOAD Module, initial step analysis of the software. One side of the plate is maintained at a potential zero so the current flows from the side of higher potential to the side of lower potential. The DC current load is defined in the Load Module, step-1 analysis in ABAQUS. The load is applied to one side at all nodes. The model is meshed using a mesh with the high density of elements near the tip of the crack.

A DC current of $1 \mathrm{Am}$ is applied at all the nodes at the higher potential side and the current is allowed to flow from one side to the other. The flow of the electrical current through a conductor is perturbed by the presence of the crack. The Job Module in ABAQUS allows writing input file for the model and then submits it for full finite element analysis. By using the Visualization Module, the results can be displayed in 
different contour plots that help in understanding the electrical current distribution in the plate.

The electrical current distribution at the tip of the crack and around the crack is obtained from the contour plot as shown in Figure 12.

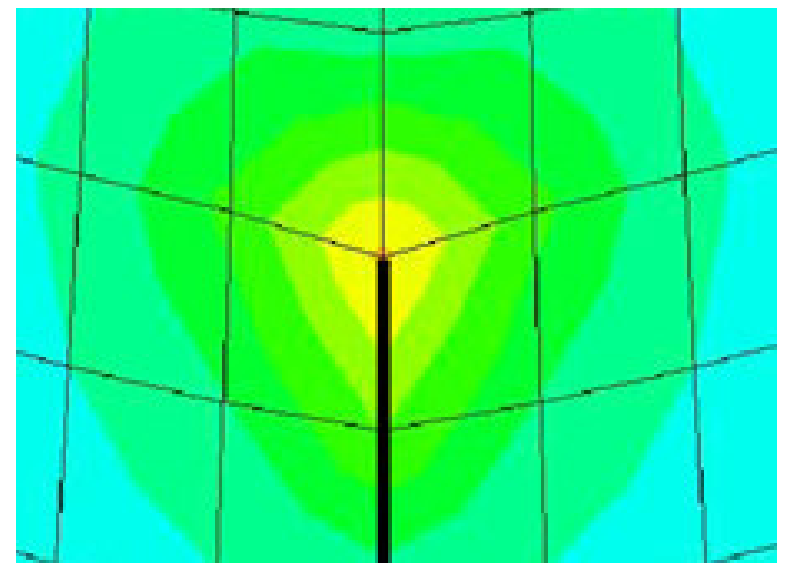

Figure 12: Electrical Current Density of the crack before applying a mechanical load

From the electrical current distribution contour plot, The electric current density at the tip of the crack is very high compared to other areas of the plate and the values of the electric current density gradually decreases away from the crack tip section of the crack. The reason behind the change in the electrical current distribution is that the current covers more area before reaching the tip of the crack. If there is no disturbance or crack, the current just flows across, thus giving the elements of the plate a low value of Electrical Current Density, unlike the tip of the crack where the current accumulates and thus giving the elements there a high value of Electrical Current Density.

It is very important to study the change in the electrical current density due to the change in the crack size and dimensions. Usually, fatigue loading in the aircraft structures causes the crack to open, get wider and longer. Therefore, electrical analysis of the 
deformed plate is carried out to check if there is any change in the electrical current density field in the plate.

A load of $1000 \mathrm{~N}$ is applied to the plate as in a simple tension test and a full mechanical analysis is carried out in ABAQUS. Stresslstrain fields are obtained for the area around the crack. Stress and strain fields around the crack tip are shown in Figure 13 and Figure 14.

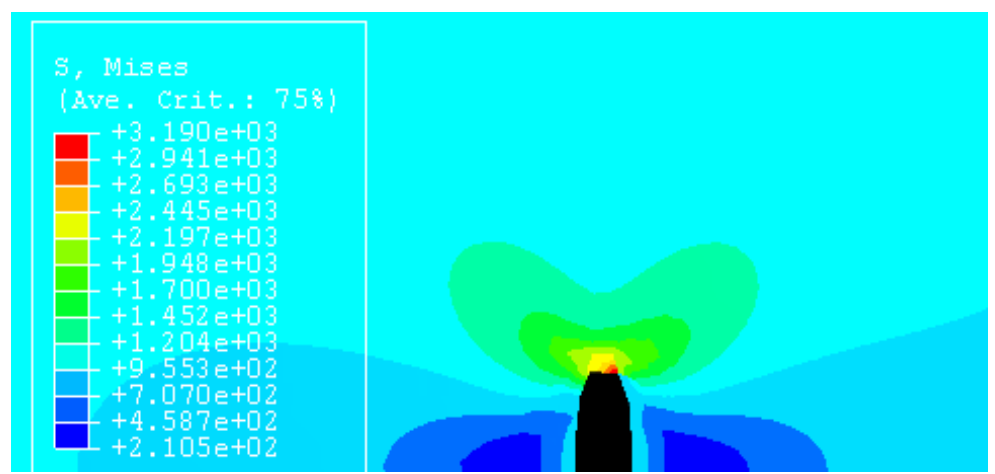

Figure 13 Stress filed around the crack tip.

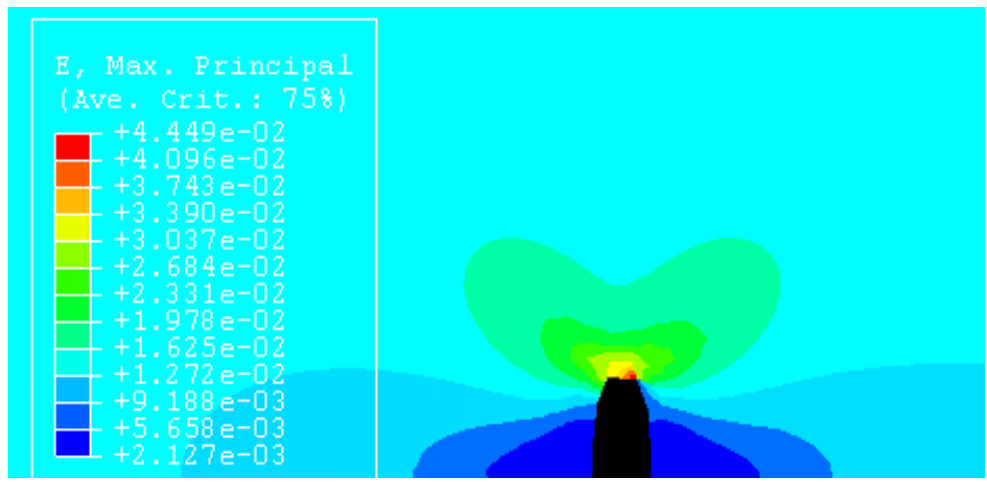

Figure 14 Strain field around the crack tip

As shown in the stress and strain field figures, the stress field will cause the crack to change its dimensions and that results in changing the crack profile. And as a result of the stress field, the nodes in the deformed model will have new coordinates. These new 
nodal coordinates for the deformed elements can be found by asking ABAQUS to give this information in the output file.

To run the electrical analysis, the new coordinates are used as the nodal coordinates of the input file for the new electrical model. The new nodal coordinates are substituted in the place of the old nodal coordinates in the input file for ABAQUS. Then the electrical analysis with the same boundary conditions, as in the old electrical model, is carried out. The new electrical current density field contour plot is obtained and analyzed. The new electrical current density field contour plot of the plate under load is shown in Figure 15.

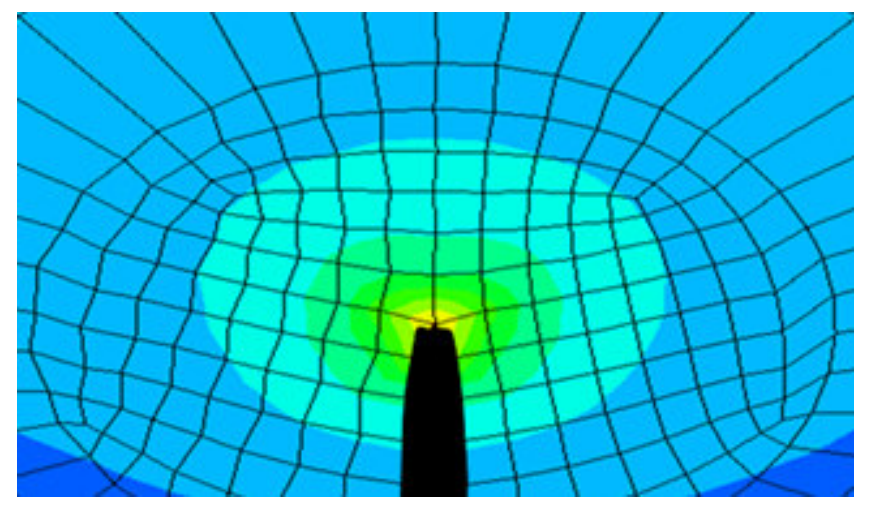

Figure 15 Crack opened up with load

For the deformed crack model, the high stress concentration in the region above the crack will cause the current density to increase in the disturbed regions around the crack and therefore the intensity of the electrical current density tends to be high. This explains the high contour plot distribution in the region above the crack. The Electrical Current Density value of the elements at the tip of the original crack is higher than that of the open crack. But, the contour plots also clearly prove the fact that the intensity of the electrical current density is much higher all around the plate. 
As a result, this change in the electrical field distribution between the two models, the original crack shape model and the deformed crack model, changes the magnetic field distribution associated with it. Therefore a different magnetic field value is obtained around the crack where the highest value will be at the crack tip. More study of the magnetic analysis are provided in details in the next sections.

\subsection{Crack Detection by Using Magnetic Flux Leakage Method}

The magnetic field produced by a current flowing in a plate can be calculated at any location above the plate as discussed in Chapter 2. By using Eq.16, the magnetic field above a plate without a crack can be calculated where the right and left ends of the plate are maintained at potential $+V_{\mathrm{o}}$ and $-V_{\mathrm{o}}$, respectively. In this section, the magnetic field for a plate without a crack will be studied and then this study will extended to calculate the change in the magnetic field due to the crack effect.

\subsubsection{The Magnetic Field from a Plate without a Crack}

The magnetic field $\vec{B}(\vec{r})$ has three components $B_{x}, B_{y}$, and $B_{z}$. We are most interested in the magnetic field component $B_{\mathrm{z}}$, since this is the component that the Hall Effect sensor can measure. For a plate without a crack, as shown in Figure 16, the magnetic field $B_{\mathrm{Z}}$ can be calculated as [34][35];

$$
B_{z}(x, y, z)=\frac{\eta_{0} t}{4 \pi} J_{0} \int_{s^{\prime}} \frac{\left(y-y^{\prime}\right)}{\left[\left(x-x^{\prime}\right)^{2}+\left(y-y^{\prime}\right)^{2}+(z)^{2}\right]^{3 / 2}} d x^{\prime} d y^{\prime}
$$




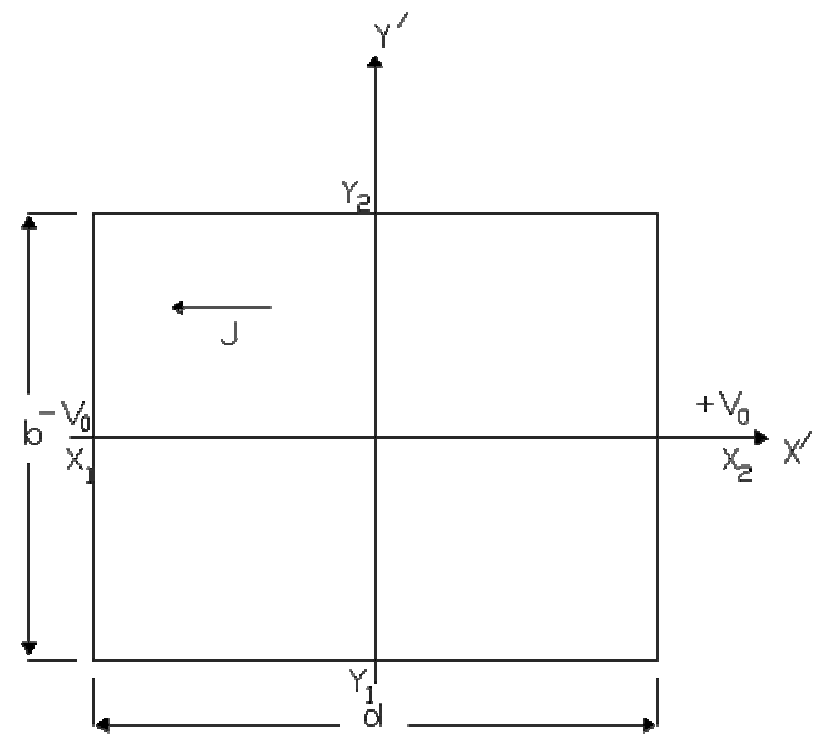

Figure 16 Plate without a crack or defect

To solve the integral we take

$$
\begin{aligned}
& \bar{Y}=y-y^{\prime} \text { and } \bar{X}=x-x^{\prime} . \\
& d \bar{Y}=-d y^{\prime} \text { and } d \bar{X}=-d x^{\prime} .
\end{aligned}
$$

E.q 18

Eq. 19

Based on Eq.18 and Eq.19, Eq.17 can be rewritten as,

$$
B_{z}(x, y, z)=\frac{\eta_{0} t}{4 \pi} J_{0} \int_{s^{\prime}} \frac{\bar{Y}}{\left[\bar{X}^{2}+\bar{Y}^{2}+z^{2}\right]^{3 / 2}} d \bar{X} d \bar{Y}
$$

Carrying out the integration in Eq.20 over $d \bar{Y}$, the magnetic field $\mathrm{B}_{\mathrm{z}}$ can be expressed as

$$
B_{z}(x, y, z)=\frac{\eta_{0} t}{4 \pi} J_{0} \int \frac{1}{\left[\bar{X}^{2}+\bar{Y}^{2}+z^{2}\right]^{1 / 2}} d \bar{X} .
$$

By letting $C^{2}=\bar{Y}^{2}+z^{2}$ then the integration part in Eq. 21 becomes

$$
\int \frac{1}{\left[\bar{X}^{2}+\bar{Y}^{2}+z^{2}\right]^{1 / 2}} d \bar{X}=\int \frac{1}{\sqrt{\bar{X}^{2}+C^{2}}} d \bar{X} .
$$

Eq. 22 
where,

$$
\int \frac{1}{\sqrt{\bar{X}^{2}+C^{2}}} d \bar{X}=\ln \left|\bar{X}+\sqrt{\bar{X}^{2}+C^{2}}\right|+g,
$$

where $g$ is a constant. By substitutes Eq.22 and Eq.23 into Eq.21:

$$
B_{z}(x, y, z)=\frac{\eta_{0} t}{4 \pi} J_{0} \ln \left|\bar{X}+\sqrt{\bar{X}^{2}+\bar{Y}^{2}+z^{2}}\right|\left|\frac{\bar{Y}_{2}}{\bar{Y}_{1}}\right|_{\bar{X}_{1}}^{\bar{X}_{2}} .
$$

Substituting Eq. 17 into Eq. 24 yields:

$$
B_{z}(x, y, z)=\frac{\eta_{0} t}{4 \pi} J_{0}\left[\begin{array}{l}
\ln \left|\left(x-x_{2}\right)+\sqrt{\left(x-x_{2}\right)^{2}+\left(y-y_{1}\right)^{2}+z^{2}}\right|- \\
\ln \left|\left(x-x_{1}\right)+\sqrt{\left(x-x_{1}\right)^{2}+\left(y-y_{1}\right)^{2}+z^{2}}\right|+ \\
\ln \left|\left(x-x_{1}\right)+\sqrt{\left(x-x_{1}\right)^{2}+\left(y-y_{2}\right)^{2}+z^{2}}\right|- \\
\ln \left|\left(x-x_{2}\right)+\sqrt{\left(x-x_{2}\right)^{2}+\left(y-y_{2}\right)^{2}+z^{2}}\right|
\end{array}\right]
$$

Eq. 25

and

$$
B_{z}(x, y, z)=\frac{\eta_{0} t}{4 \pi} J_{0}\left[\begin{array}{r}
\ln \frac{\left(x-x_{2}\right)+\sqrt{\left(x-x_{2}\right)^{2}+\left(y-y_{1}\right)^{2}+z^{2}}}{\left(x-x_{1}\right)+\sqrt{\left(x-x_{1}\right)^{2}+\left(y-y_{1}\right)^{2}+z^{2}}} \\
+\ln \frac{\left(x-x_{1}\right)+\sqrt{\left(x-x_{1}\right)^{2}+\left(y-y_{2}\right)^{2}+z^{2}}}{\left(x-x_{2}\right)+\sqrt{\left(x-x_{2}\right)^{2}+\left(y-y_{2}\right)^{2}+z^{2}}}
\end{array}\right],
$$

Eq. 26

where in the notation of Figure 16

$$
x_{1}=-d / 2, \quad x_{2}=d / 2, y_{1}=-b / 2, \text { and } y_{2}=b / 2 \text { are the related to the plate }
$$

dimensions length and width,

Thus;

$$
J_{0}=I / \text { Aer } a=I / t b,
$$

where $I$ is the current, $t$ is the plate thickness and $b$ is the plate width. 
This kind of analysis in MATLAB is used to show the magnetic field above a plate without a crack. For this case, the dc current is provided as $10 \mathrm{Am}$, plate dimensions are $(0.2 \times 0.2 \times .003)$ meter as $b, d$ and $t$, respectively. The magnetic field above the plate with a distance $\mathrm{z}=.001$ meter from the surface of the plate is to be measured. The size of the element that is to be scanned at its nodes is $.0005 \times$ x .0005 meter. The magnetic field above the crack is shown in Figure 17.

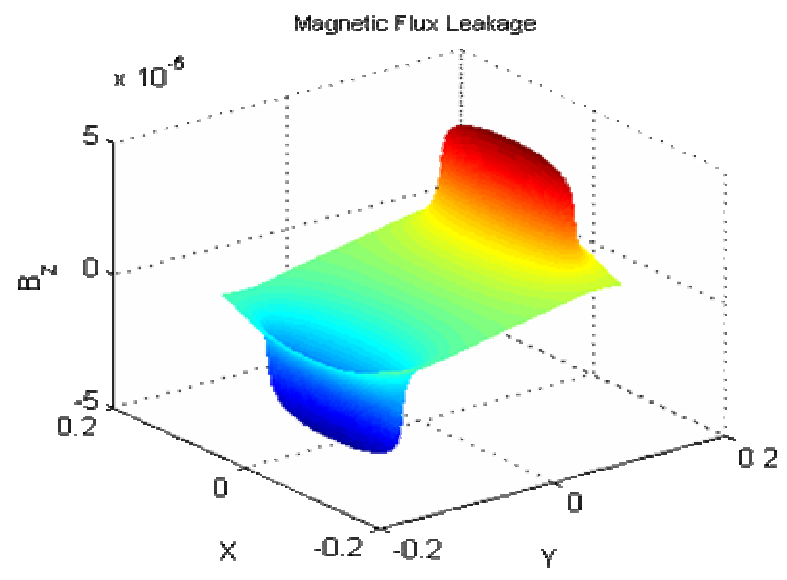

Figure 17 Magnetic field above a plate without a crack at $\mathrm{z}=0.001 \mathrm{~m}$

In Figure 17, the huge magnetic field produced by the plate edges can be seen, where the red color represents the positive direction of the magnetic field and the blue color represents the negative direction. Figure 18 shows the magnetic field contour lines above the middle of the plate. The change in the magnetic filed due to the edges in this area is linear which is expected to change in the case of a crack existence. 


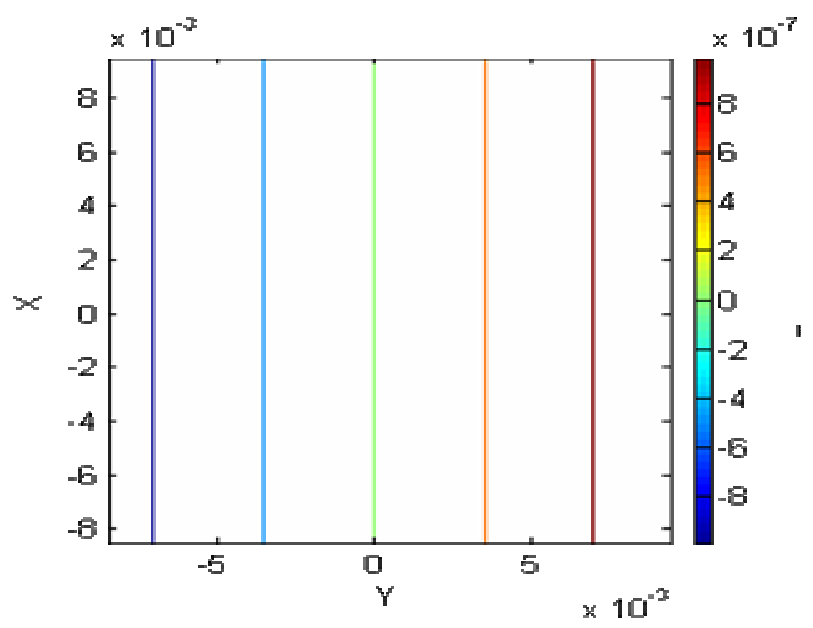

Figure 18 Magnetic field contour lines above the middle of the plate with no crack.

\subsubsection{The Magnetic Field from a Plate with a Crack}

In the presence of a crack in the plate, the current distribution will change in the plate with the highest value around the crack tip. This will result in changing the magnetic field above the plate and around the crack location. As mentioned before, the normal component of the magnetic field, Bz, is the quantity of interest. The law of BiotSavart can be used again to calculate this component. In this section, the equations for calculating $\mathrm{Bz}$ will be provided and then these equations can be used in the modeling analysis. The change in the magnetic field will result from both the plate edges and the crack. In this study, the surface crack has a length, L, width, W, and depth, D.

Figure 19 shows a schematic diagram of a plate with a surface defect in the middle of the plate. 


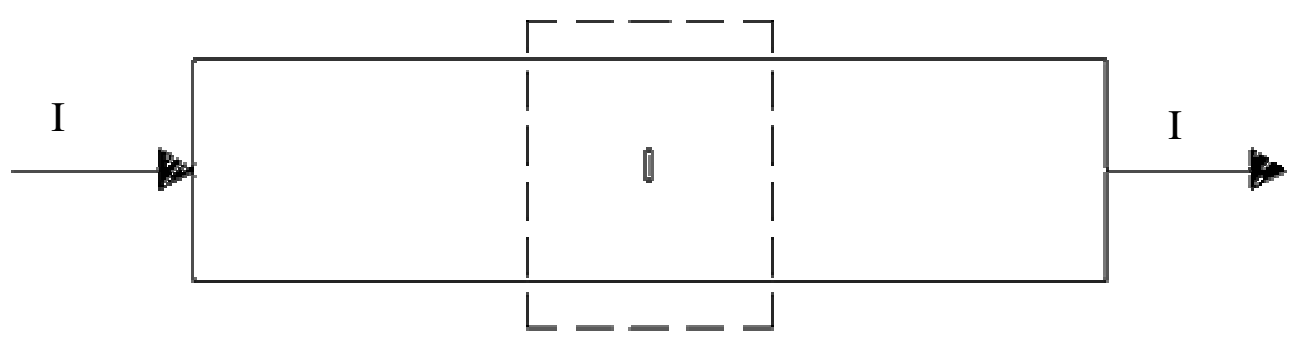

( a )

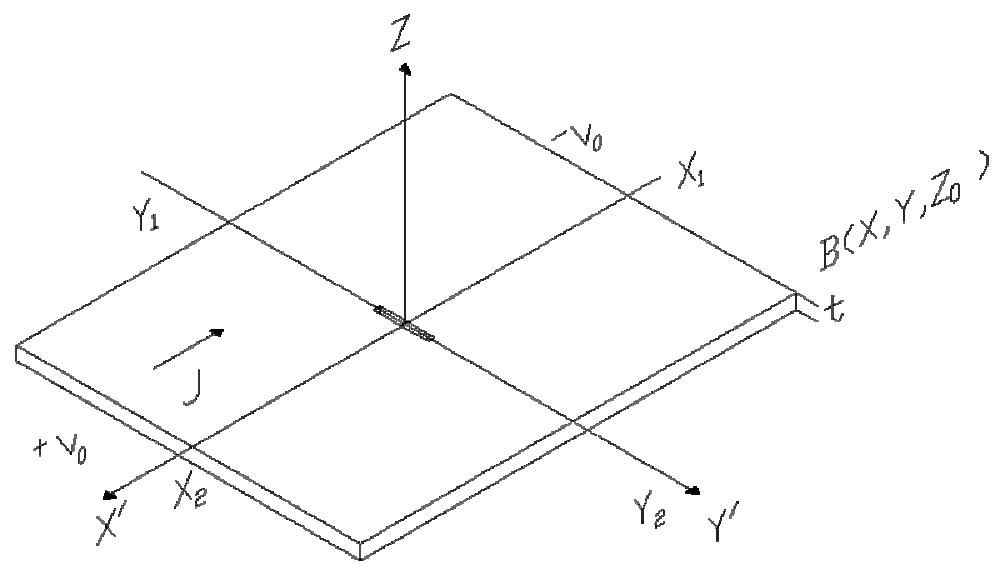

(b)

Figure 19 Plate with a surface crack.

Recalling the law of Biot-Savart;

$$
\vec{B}(\vec{r})=\frac{\mu_{0}}{4 \pi} \int_{v^{\prime}} \frac{J\left(\overrightarrow{r^{\prime}}\right) \times\left(\vec{r}-\vec{r}^{\prime}\right)}{\left|\vec{r}-\overrightarrow{r^{\prime}}\right|^{3}} d x^{\prime} d y^{\prime} d z^{\prime} \cdot
$$

The volume of interest in our analysis is homogeneous and isotropic and does not contain sources of current. As a result, the magnetic field reduces to the evaluation of the integral over the surface bounding the volume. And for a two-dimensional current distribution flowing in a thin plate located in the $x$, 'y plane, the integral over $d z^{\prime}$ ' reduces 
to a multiplicative factor $t$ for the thickness in the $z$ '-direction and in this case Eq.28 becomes an integral over the surface, where,

$$
\vec{B}(\vec{r})=\frac{\mu_{0} t}{4 \pi} \int_{s^{\prime}} \frac{J\left(\overrightarrow{r^{\prime}}\right) \times\left(\vec{r}-\vec{r}^{\prime}\right)}{\left|\vec{r}-\overrightarrow{r^{\prime}}\right|^{3}} d x^{\prime} d y^{\prime}
$$

It is important to note that the planar surfaces do not contribute to the magnetic field component $\mathrm{B}_{\mathrm{z}}$. The only surfaces in the plate that can contribute to the normal magnetic field are the surfaces whose normal is perpendicular to the $\mathrm{z}$ coordinate. That means the thin surfaces that define the thickness of the plate and the depth of the crack are the only surfaces that we need to consider.

In mathematical equations, this can be expressed by rewriting Eq. 29 as the following:

$$
\vec{B}(\vec{r})=\frac{\mu_{0} t_{c}}{4 \pi} \int_{\text {crack }} \frac{J\left(\overrightarrow{r^{\prime}}\right) \times\left(\vec{r}-\overrightarrow{r^{\prime}}\right)}{\left|\vec{r}-\overrightarrow{r^{\prime}}\right|^{3}} d x^{\prime} d y^{\prime}+\frac{\mu_{0} t_{p}}{4 \pi} \int_{\text {plate }} \frac{J\left(\overrightarrow{r^{\prime}}\right) \times\left(\vec{r}-\vec{r}^{\prime}\right)}{\left|\vec{r}-\overrightarrow{r^{\prime}}\right|^{3}} d x^{\prime} d y^{\prime}
$$

where $t_{c}$ and $t_{p}$ are the crack thickness and the plate thickness, respectively.

Eq. 30 can be written in $x^{\prime}, y^{\prime}$ and $z^{\prime}$ coordinates as,

$$
\begin{aligned}
B_{z}(x, y, z)= & \frac{\eta_{0} t_{c}}{4 \pi} \int_{\text {crack }} \frac{J_{y^{\prime}}\left(x^{\prime}, y^{\prime}\right)\left(y_{c}-y^{\prime}\right)-J_{x^{\prime}}\left(x^{\prime}, y^{\prime}\right)\left(x_{c}-x^{\prime}\right)}{\left[\left(x_{c}-x^{\prime}\right)^{2}+\left(y_{c}-y^{\prime}\right)^{2}+\left(z-z^{\prime}\right)^{2}\right]^{3 / 2}} d x^{\prime} d y^{\prime}+ \\
& \frac{\eta_{0} t_{p}}{4 \pi} \int_{\text {plate }} \frac{J_{x^{\prime}}\left(x^{\prime}, y^{\prime}\right)\left(y_{p}-y^{\prime}\right)-J_{y^{\prime}}\left(x^{\prime}, y^{\prime}\right)\left(x_{p}-x^{\prime}\right)}{\left[\left(x_{p}-x^{\prime}\right)^{2}+\left(y_{p}-y^{\prime}\right)^{2}+\left(z-z^{\prime}\right)^{2}\right]^{3 / 2}} d x^{\prime} d y^{\prime}
\end{aligned},
$$

Eq. 31

where $x_{c}$ and $y_{c}$ are related to the crack dimensions and $x_{p}$ and $y_{p}$ are related to the plate dimensions. 
Since the $y^{\prime}$ component of the current density $J_{y^{\prime}}\left(x^{\prime}, y^{\prime}\right)$ is zero, the $x^{\prime}$-component of the current density $J_{x^{\prime}}\left(x^{\prime}, y^{\prime}\right)$ is the constant $J_{0}$ in Eq. 27 , and by taking $z^{\prime}=0$, then Eq. 31 reduces to

$$
\begin{aligned}
B_{z}(x, y, z)= & \frac{\eta_{0} t_{p}}{4 \pi} J_{0} \int_{\text {plate }} \frac{\left(y_{p}-y^{\prime}\right)}{\left[\left(x_{p}-x^{\prime}\right)^{2}+\left(y_{p}-y^{\prime}\right)^{2}+(z)^{2}\right]^{3 / 2}} d x^{\prime} d y^{\prime}- \\
& \frac{\eta_{0} t_{c}}{4 \pi} J_{0} \int_{\text {crack }} \frac{\left(y_{c}-y^{\prime}\right)}{\left[\left(x_{c}-x^{\prime}\right)^{2}+\left(y_{c}-y^{\prime}\right)^{2}+(z)^{2}\right]^{3 / 2}} d x^{\prime} d y^{\prime}
\end{aligned}
$$

The integration over the plate and the crack edges as in the previous section can be represented as follows:

$$
\begin{gathered}
B_{z}(x, y, z)=\frac{\eta_{0} t_{p}}{4 \pi} J_{0}\left[\begin{array}{r}
\ln \frac{\left(x-x_{p_{2}}\right)+\sqrt{\left(x-x_{p_{2}}\right)^{2}+\left(y-y_{p_{1}}\right)^{2}+z^{2}}}{\left(x-x_{p_{1}}\right)+\sqrt{\left(x-x_{p_{1}}\right)^{2}+\left(y-y_{p_{1}}\right)^{2}+z^{2}}} \\
+\ln \frac{\left(x-x_{p_{1}}\right)+\sqrt{\left(x-x_{p_{1}}\right)^{2}+\left(y-y_{p_{2}}\right)^{2}+z^{2}}}{\left(x-x_{p_{2}}\right)+\sqrt{\left(x-x_{p_{2}}\right)^{2}+\left(y-y_{p_{2}}\right)^{2}+z^{2}}}
\end{array}\right]- \\
\frac{\eta_{0} t_{c}}{4 \pi} J_{0}\left[\begin{array}{r}
\ln \frac{\left(x-x_{c 2}\right)+\sqrt{\left(x-x_{c 2}\right)^{2}+\left(y-y_{c 1}\right)^{2}+z^{2}}}{\left(x-x_{c 1}\right)+\sqrt{\left(x-x_{c 1}\right)^{2}+\left(y-y_{c 1}\right)^{2}+z^{2}}} \\
+\ln \frac{\left(x-x_{c 1}\right)+\sqrt{\left(x-x_{c 1}\right)^{2}+\left(y-y_{c 2}\right)^{2}+z^{2}}}{\left(x-x_{c 2}\right)+\sqrt{\left(x-x_{c 2}\right)^{2}+\left(y-y_{c 2}\right)^{2}+z^{2}}}
\end{array}\right]
\end{gathered}
$$

Eq. 33

Where in this notation of Figure $20 x_{p 1}=-d_{p} / 2, x_{p 2}=d_{p} / 2, y_{p 1}=-b_{p} / 2$, and $y_{p 2}$ $=b_{p} / 2$ are related to the plate dimensions. And $x_{c l}=-d_{c} / 2, x_{c 2}=d_{c} / 2, y_{1}=-b_{c} / 2$, and $y_{c 2}=b_{c} / 2$ are related to the crack dimensions. 


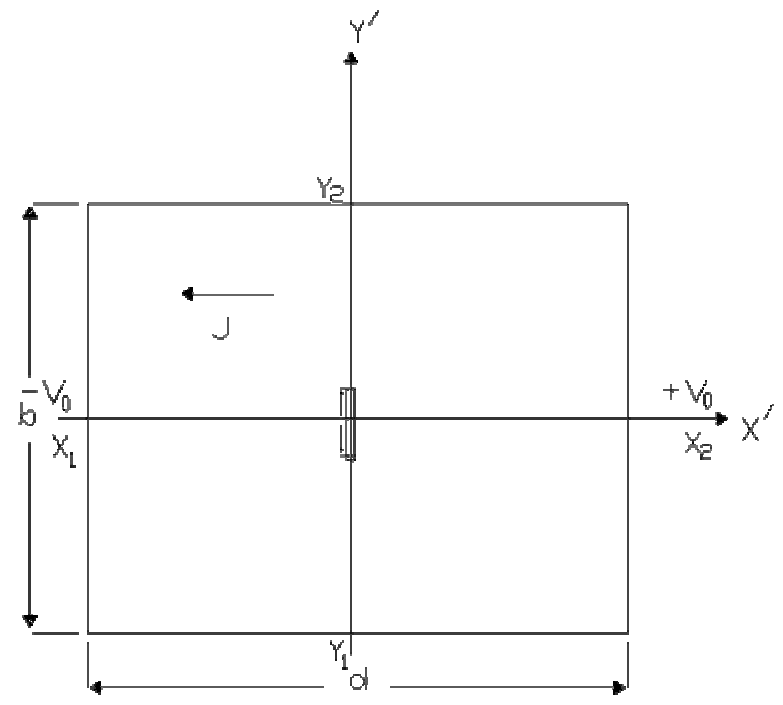

Figure 20 Plate with a crack or defect in the center

This analysis is carried out as in the last section. The dc current is taken as 10 Am, the plate dimensions are $(0.2 \times 0.2 \times .003)$ meter as $b, d$ and $t$, respectively. The magnetic field above the plate is measured with a distance $\mathrm{z}=0.001$ meter from the surface of the plate and the step size is 0.00005 meter. In this case the crack dimensions are $(0.006 \times 0.002 \times .001)$ meter. The magnetic field above a plate with this crack dimensions is shown in Figure 21, Figure 22, Figure 23, and Figure 24. 


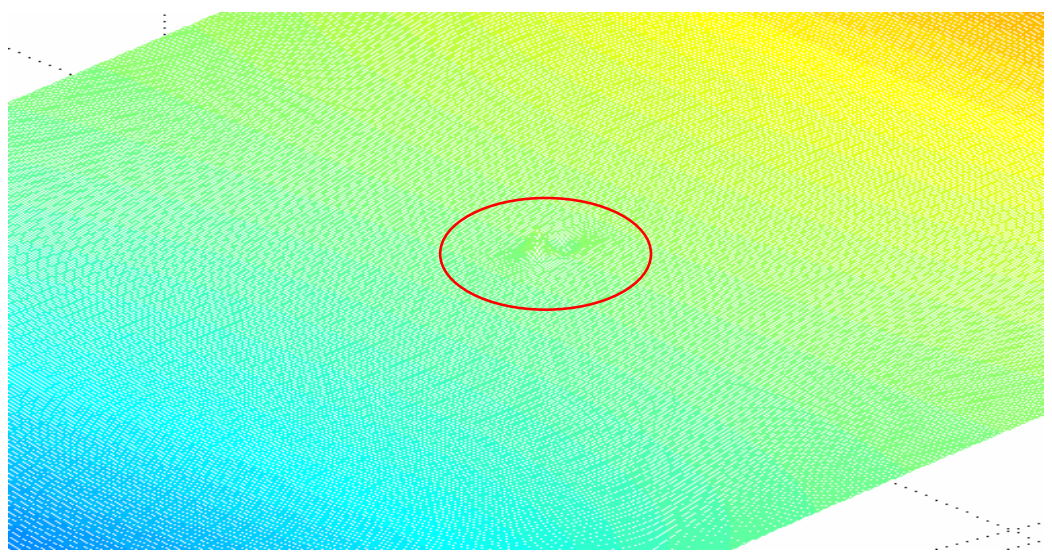

Figure 21 Magnetic field above a plate with a $(0.006 \times 0.002 \times .001)$ meter dimensions crack.

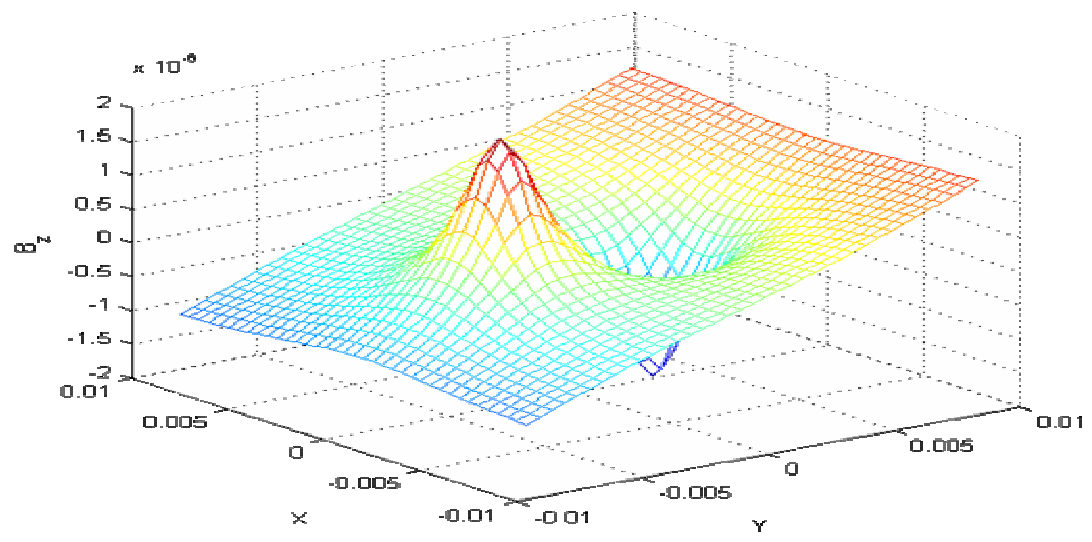

Figure 22 Close up look at the field above the crack

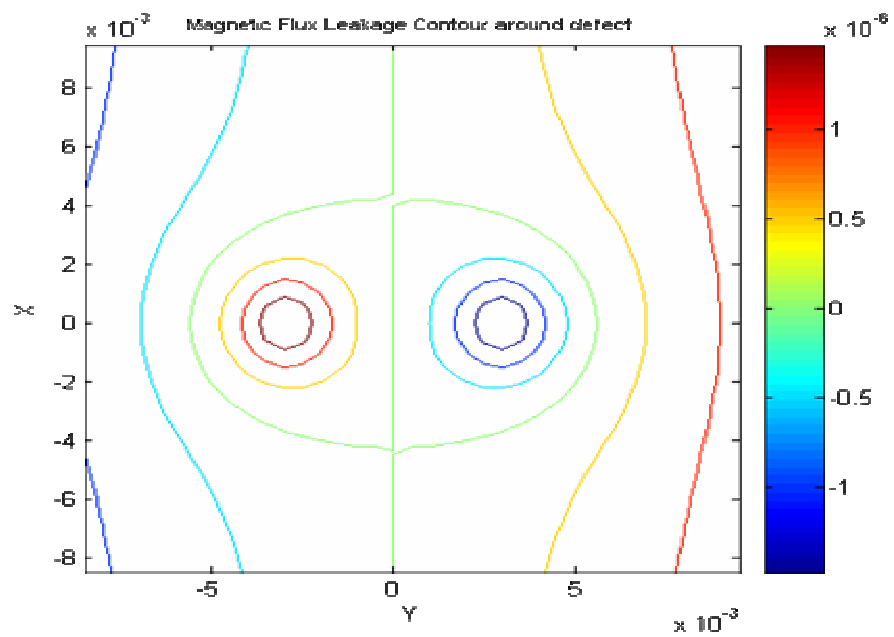

Figure 23 Magnetic field contour lines around the crack location 


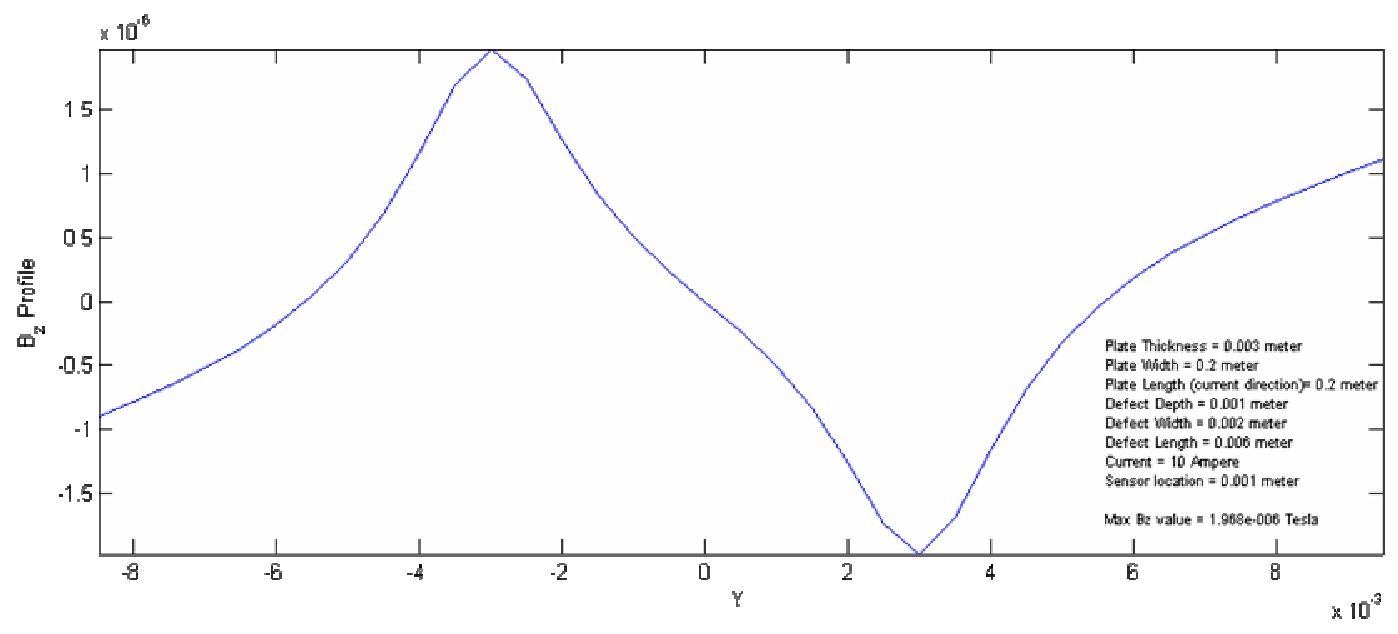

Figure 24 Normal Magnetic Flux Signal above the crack at $x=0.0$

In this case, where there is a crack in the plate, a change in the magnetic field is noticed above the area where the crack is located. This is why by using this method, the crack location can be detected and information about the crack profile can be obtained. The change in the magnetic field produced by the crack is very small compared to the magnetic fields produced by the plate edges as shown in Figure 21. This problem is discussed in detail in section 4.3.

From Figure 18 and Figure 23, the big difference in the magnetic field distribution around the crack location can be observed. Figure 23 clearly indicates that the maximum magnetic field is located at the tip of the crack. In this case, there are two magnetic fields with the same magnitudes but opposite directions at both tips. And this is also clear in Figure 24, where the data from this figure will be used in training the neural network.

In the Following subsections, the effect of different factors on the magnetic field above the crack location is discussed. 


\subsubsection{Effect of the Current Flowing Thru the Plate}

To study the effect of changing the current that flows in the plate, all other variables are fixed and the same analysis is run while changing the current from $0.5 \mathrm{Am}$ to $10 \mathrm{Am}$ in steps of $0.5 \mathrm{Am}$. In this case the sensor location is at $\mathrm{z}=.001$ and plate dimensions are $(0.2 \times 0.2 \times 0.002)$ meter and the crack dimensions are taken as $(0.008 \times 0.002 \times 0.002)$ meter. Figure 25 shows the relation between the magnetic field and the current is linear. Having more current to flow thru the plate will increase the strength of the magnetic signal and this in turn helps us detect the signal over the crack. The sensor will be able to detect the signal with better resolution in the case of stronger magnetic signals.

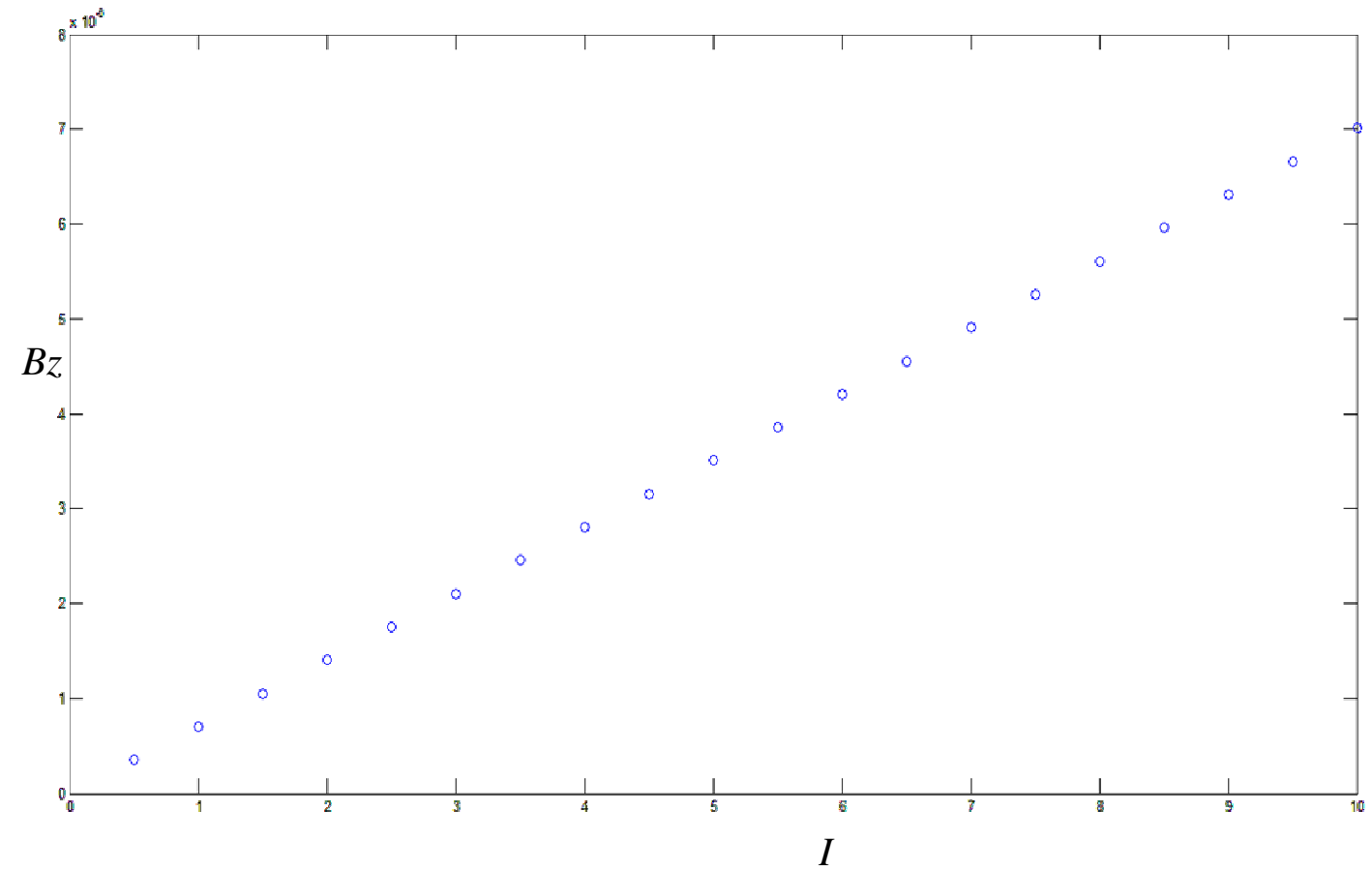

Figure 25 Effect of the current flowing thru the plate. 


\subsubsection{Effect of Sensor Location.}

To study the effect of changing the sensor location above the crack on the signal strength, all other variables are fixed and run with the same analysis by changing the sensor position from $\mathrm{z}=0.0005$ to $\mathrm{z}=0.0050 .5$ meter. In this case the current $\mathrm{I}=10 \mathrm{Am}$, and plate dimensions are $(0.2 \times 0.2 \times 0.002)$ meter and the crack dimensions are taken as $(0.008 \times 0.002 \times 0.002)$ meter. Figure 26 shows that the relation between the magnetic field and the sensor location is not linear, and by increasing the sensor location above the plate current the magnetic field is decreasing rapidly. This is consistent with the Biot-Savart Law. Thus, the sensor will be able to detect signals with better resolution when it is closer to the plate.

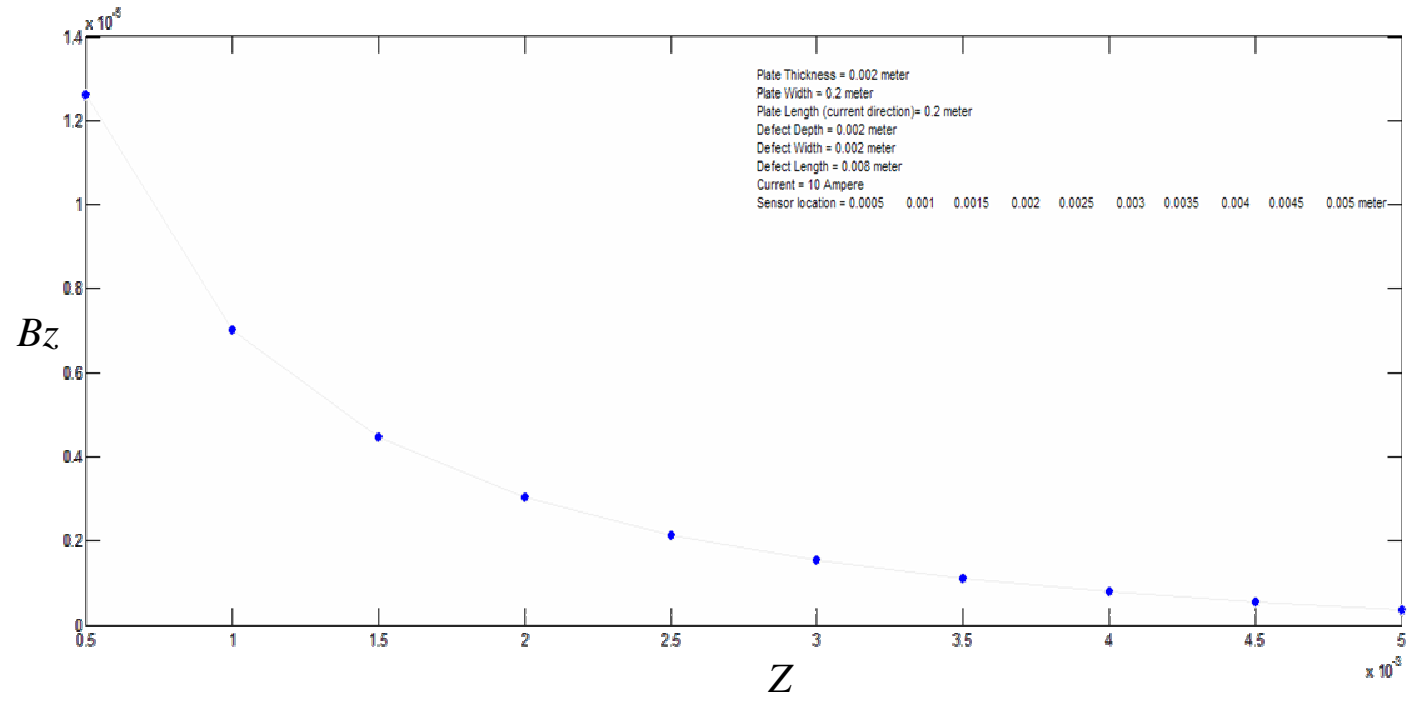

Figure 26 Effect of sensor location

\subsubsection{Effect of Crack Profile}

It is important to study the effect of the crack profile on the magnetic field above the crack; changing the crack dimensions will change the magnitude and shape of the 
magnetic flux signal above the crack. This information will help us understand how each dimension of the crack will affect the magnetic field signal. In this section, the effect of the crack length, width, and depth will be shown on the magnetic signal where all other variables are fixed.

\subsection{Crack Length Factor}

Under fatigue loading, cracks tend to grow in normal directions to the surface, where detection of the crack tip will give an indication of the critical failure of the structure. By using this method we can monitor the crack length and decide the maintenance cycles for the whole structure.

To study this factor, we run many cases where the length of the crack is changed and the other dimensions are fixed. Figure 27 shows different magnetic signals above the crack area, and Figure 28 shows the magnetic contour lines around the crack tip.

In Figure 27 we see the clear change in the magnetic signal around the crack; where the maximum magnetic values are at the crack at both tips. From Figure 28, it is clear that at the center of the crack, the magnetic field is zero; this information helps to determine the crack center and the crack location. Also noticed that propagation of the crack length increased the magnetic signal at the crack tip, as shown in Figure 29.

The magnetic field away from the crack tips is not close to zero and that is because of the effect of the huge magnetic fields produced by the plate edges. This will cause a serious problem for the detection of the crack given that the sensor may not detect the peak value at the crack tip and detect the magnetic field away from the crack tip that was produced by the edges. A way needs to be found to cancel this effect or at least minimize it around the crack area. Section 4.3 will deal with this problem. 


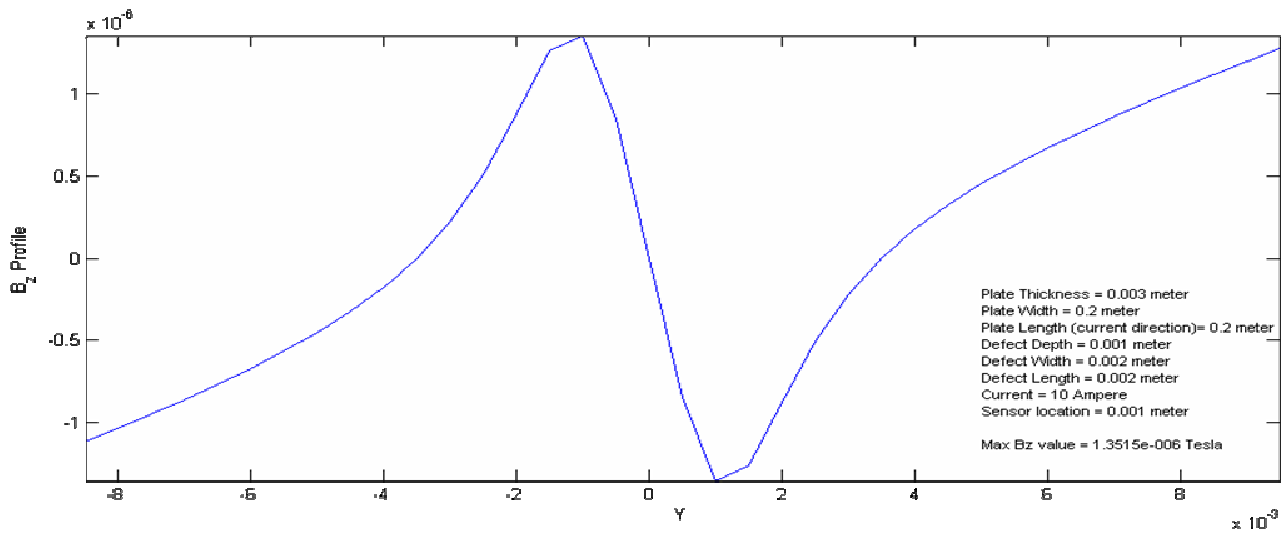

(a)

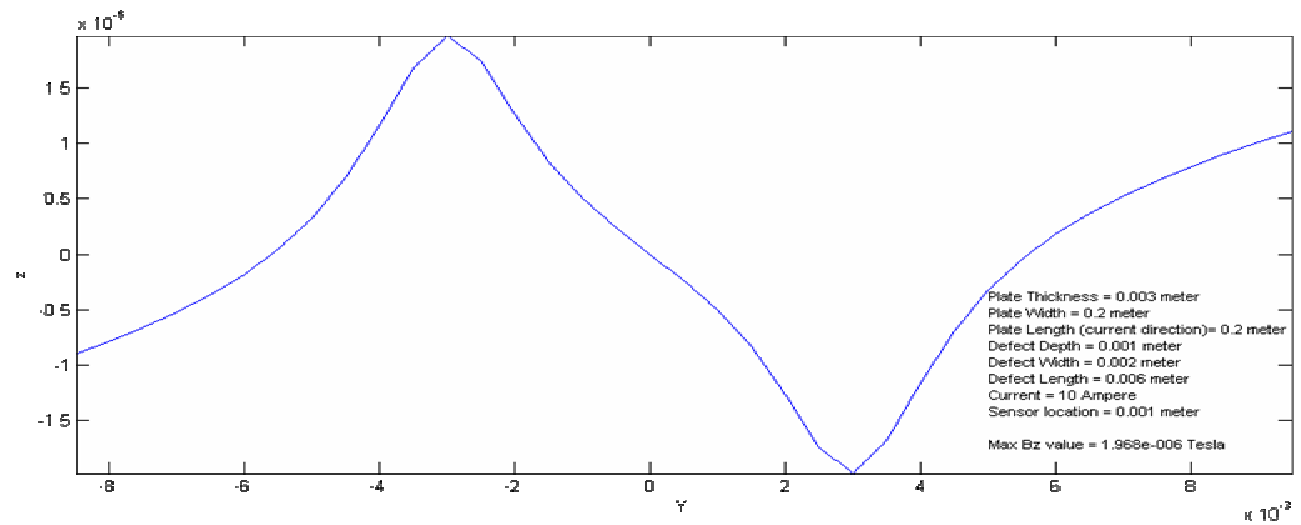

(b)

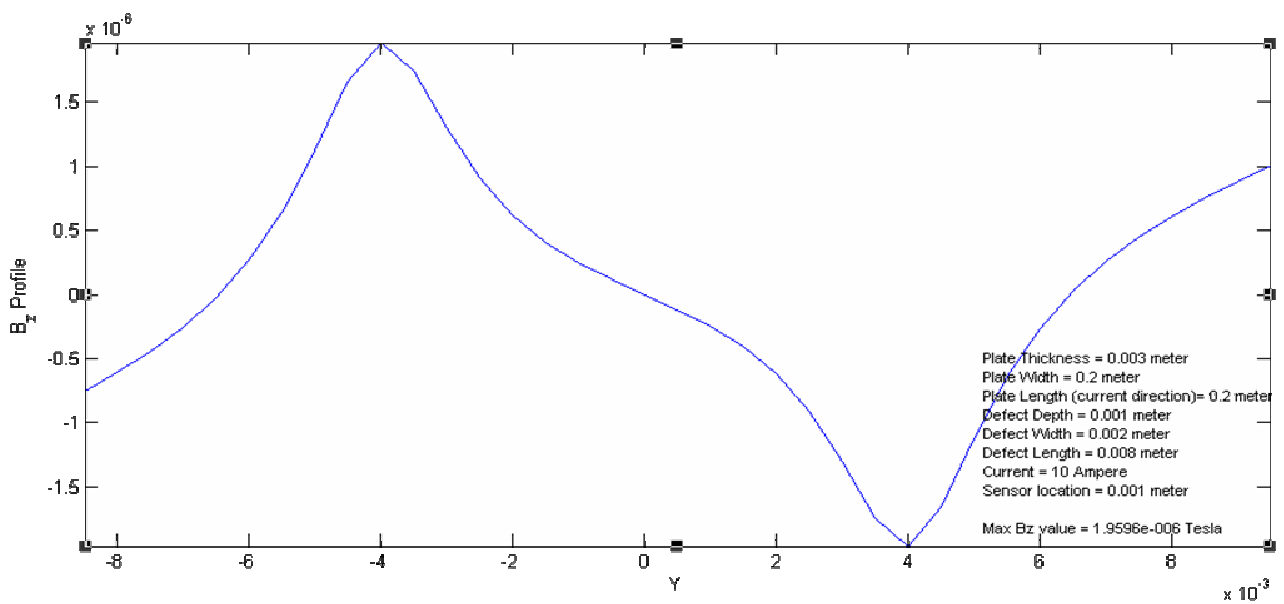

(c)

Figure 27 Crack length factor. Where, in ( a ) $\mathrm{L}=\mathbf{0 . 0 0 2} \mathbf{~ m}$,

( b ) $\mathrm{L}=0.004 \mathrm{~m}$, and ( c ) $\mathrm{L}=0.006 \mathrm{~m}$. 


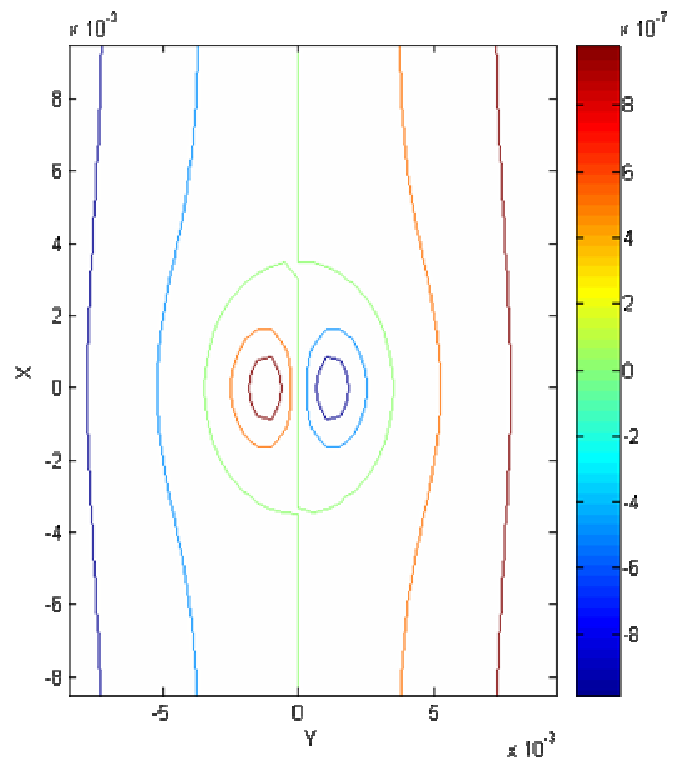

( a )

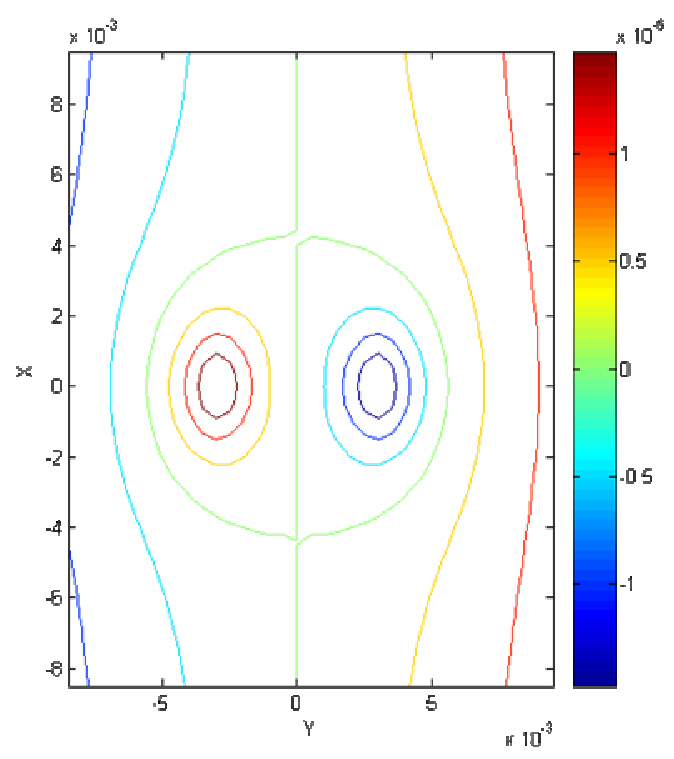

( b )

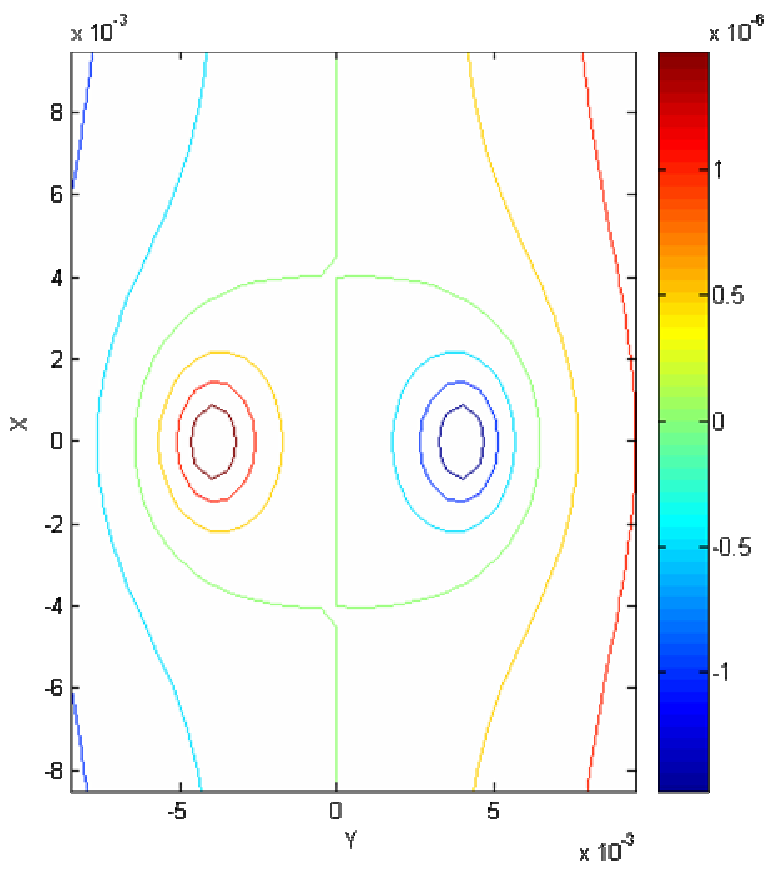

(c)

Figure 28 Magnetic field contour lines around the crack location. Where, in ( a ) $\mathrm{L}=\mathbf{0 . 0 0 2} \mathrm{m}$, (b ) $\mathrm{L}=0.004 \mathrm{~m}$, and ( c ) $\mathrm{L}=\mathbf{0 . 0 0 6} \mathrm{m}$. 


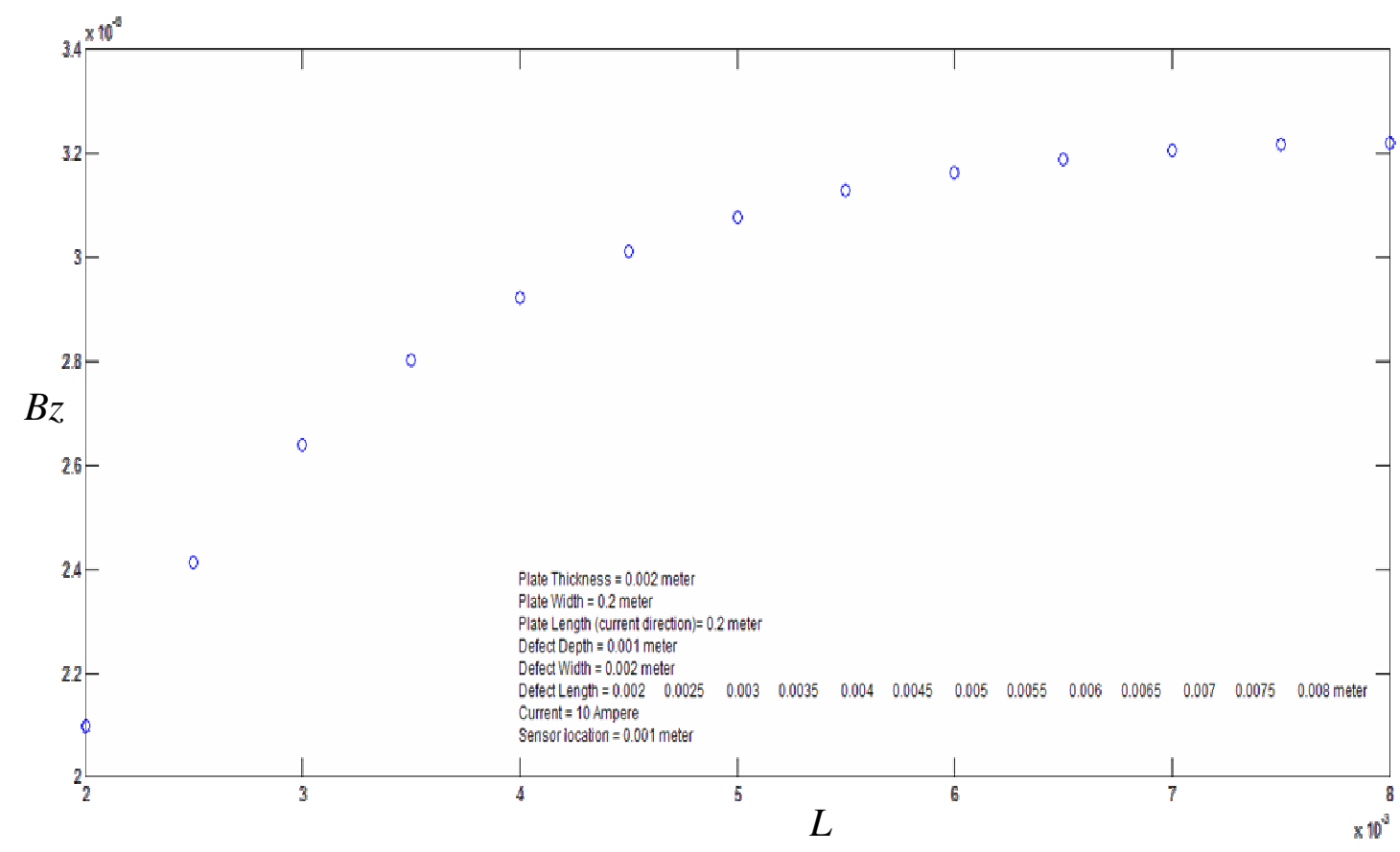

Figure 29 Crack length effect on the magnetic field at the crack tip

\subsection{Crack Width Factor}

The same analysis is carried out to study the change in the crack width on the magnetic field around the crack. To study this factor, many cases will be run where the width of the crack is changed while fixing the other dimensions. Figure 30 shows different magnetic signals above the crack area, and Figure 31 shows the contour lines around the crack. Figure 32 shows the relation between the magnetic field and the increment in the width. The effect in the magnitude of the magnetic field is clear and strong, but the shape of the signal is less than the effect in the case of the length factor. In Figure 32, the magnetic field is calculated at the crack tip. 


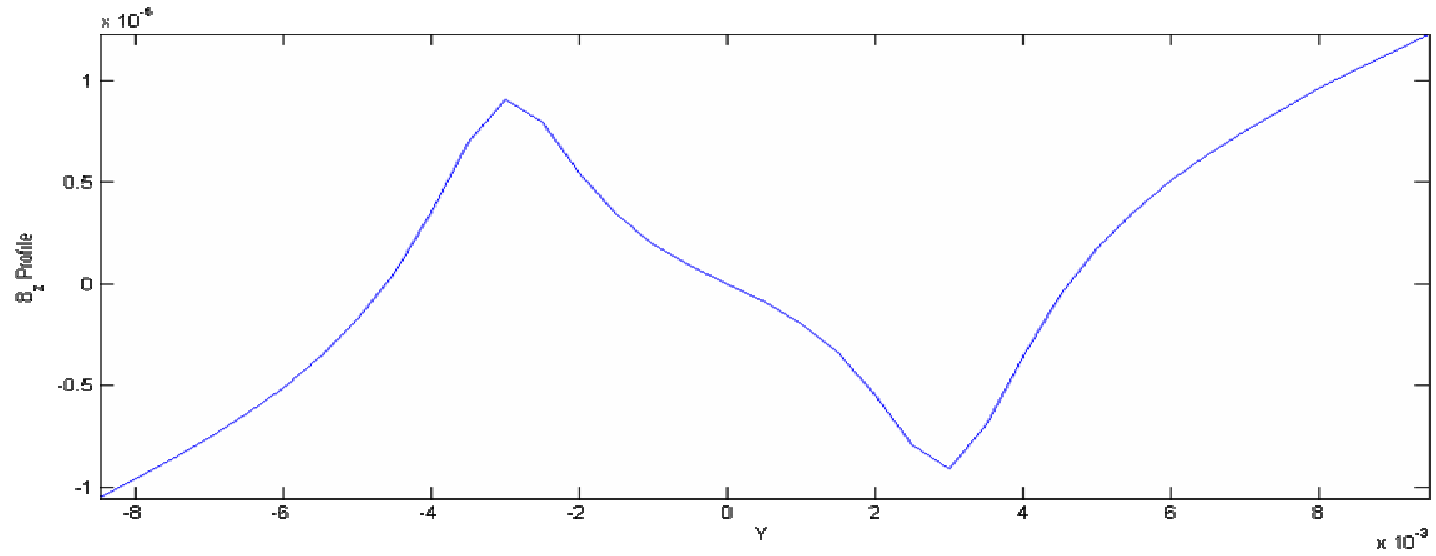

( a )

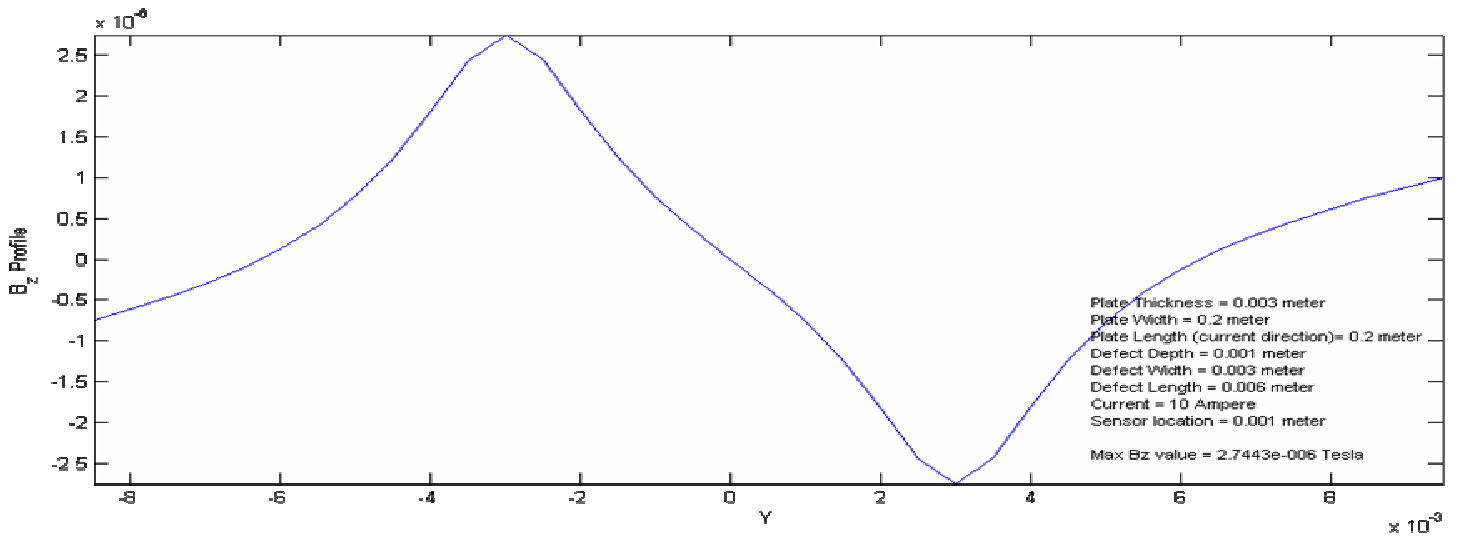

( b )

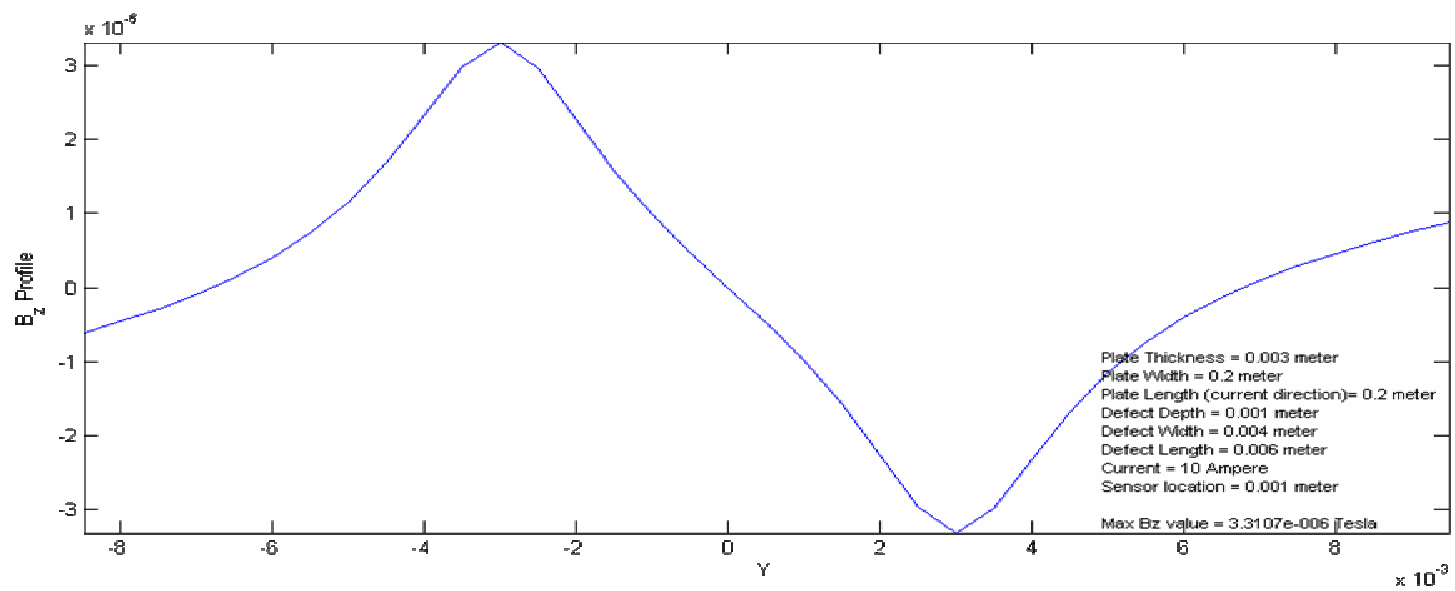

( c)

Figure 30 Crack width factor. Where, in ( a ) $w=0.001 \mathrm{~m}$,

( b ) $\mathrm{w}=0.003 \mathrm{~m}$, and ( c ) $\mathrm{w}=0.004 \mathrm{~m}$. 


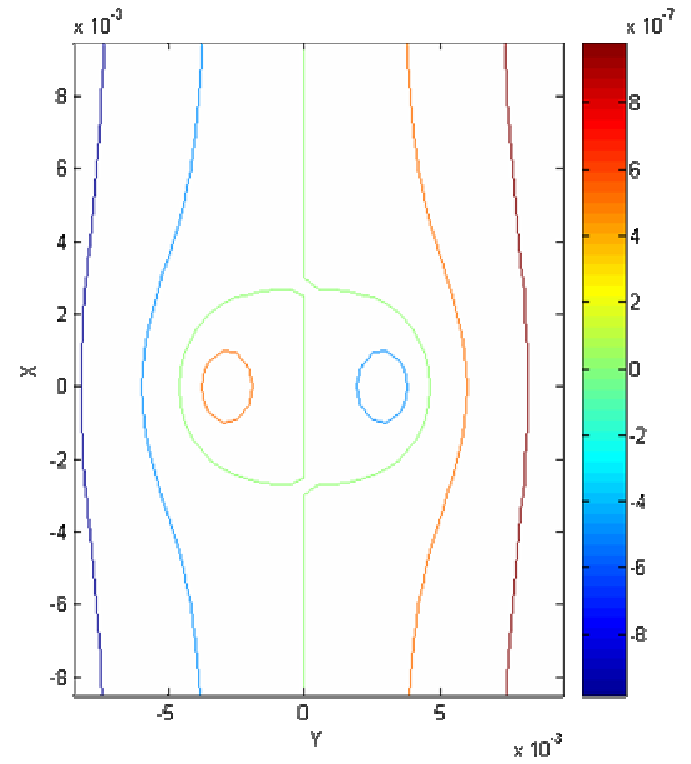

( a )

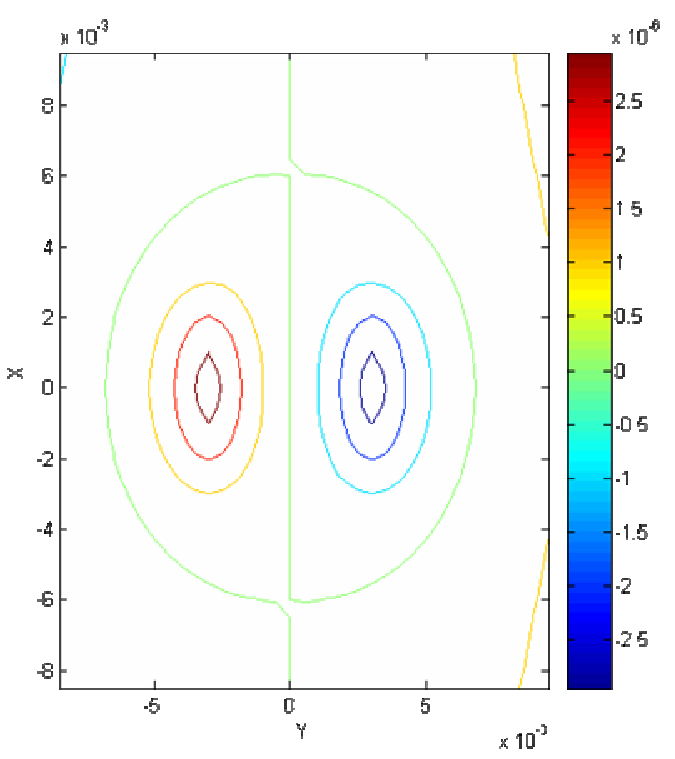

( b )

Figure 31 Magnetic field contour lines around the crack location. Where, in ( a ) w = 0.001 $\mathrm{m}$, and $(\mathrm{b}) \mathrm{w}=\mathbf{0 . 0 0 4} \mathrm{m}$.

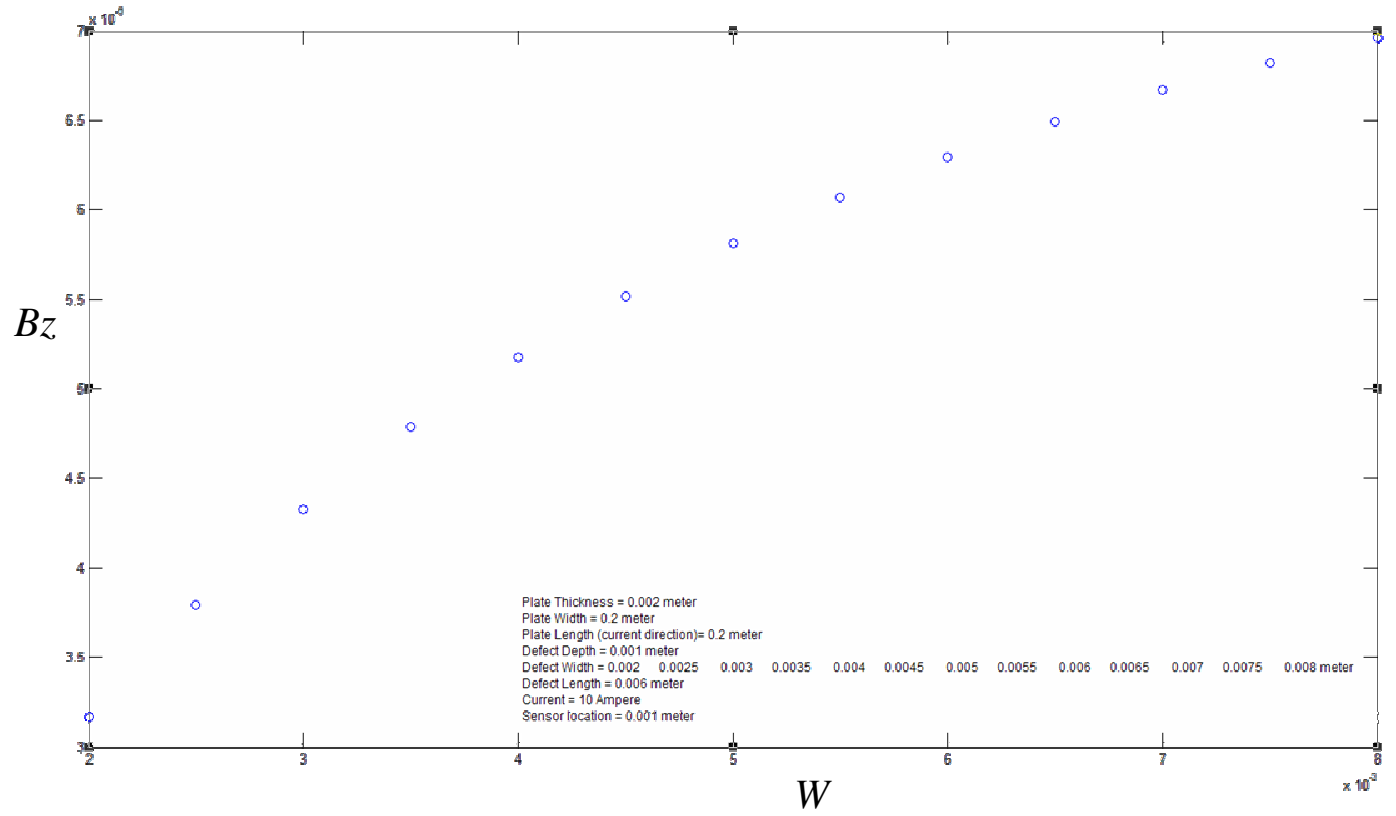

Figure 32 Crack width effect on the magnetic field at the crack tip 


\subsection{Crack Depth Factor}

The same analysis is carried out to study the change in the crack depth on the magnetic field around the crack. To study this factor, many cases where run while changing the depth of the crack and fixing the other dimensions. Figure 33 shows different magnetic signals above the crack area and Figure 34 shows the contour lines around the crack. Figure 35 shows the relation between the magnetic field and the increment in the crack depth. The effect in the magnitude of the magnetic field is clear but the shape of the signal is less than in the case of the length factor. For the analysis in Figure 35, the magnetic field is calculated at the crack tip.

In Figure 36, the magnetic field produced by the plate edges is very high compared to the signal from the crack, while the same effect is small when the crack depth is very large and the crack is deep in the plate, Figure 37 . This means that to detect cracks with very small depth size, it is important to use high sensitive sensors and search around the crack area where there is a crack, or find a way to eliminate this edge effect. All this discussion leads to the point in the study where the need to minimize the effect of the plate on the magnetic field signal is crucial. 


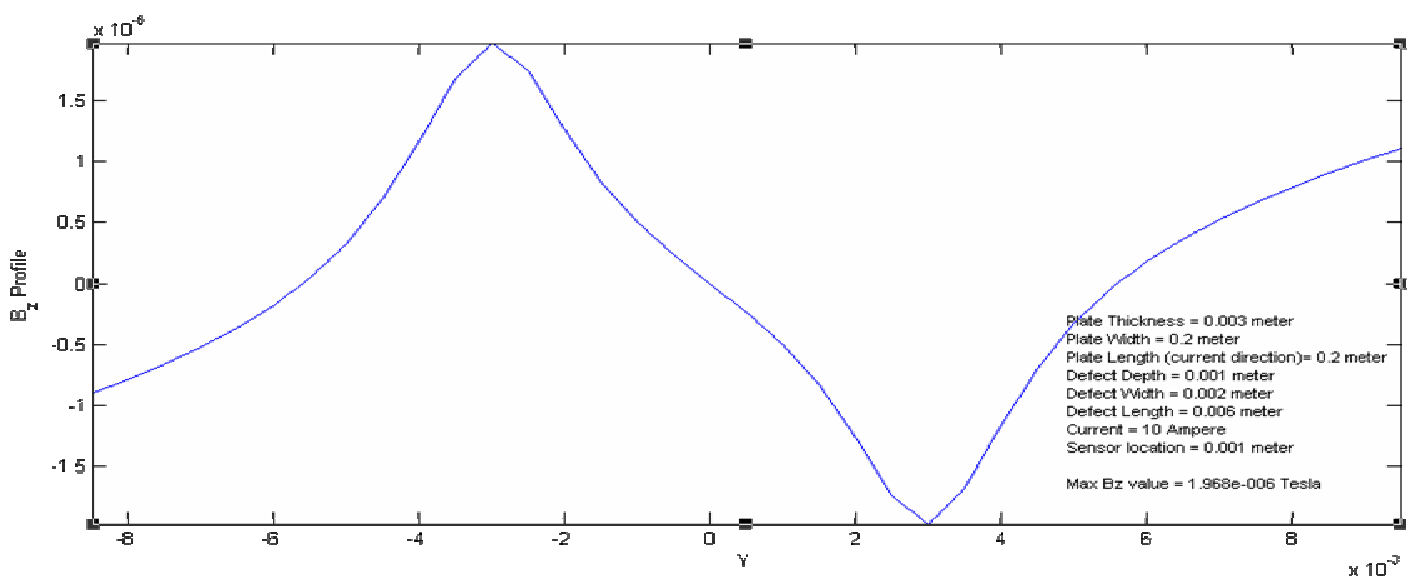

(a)

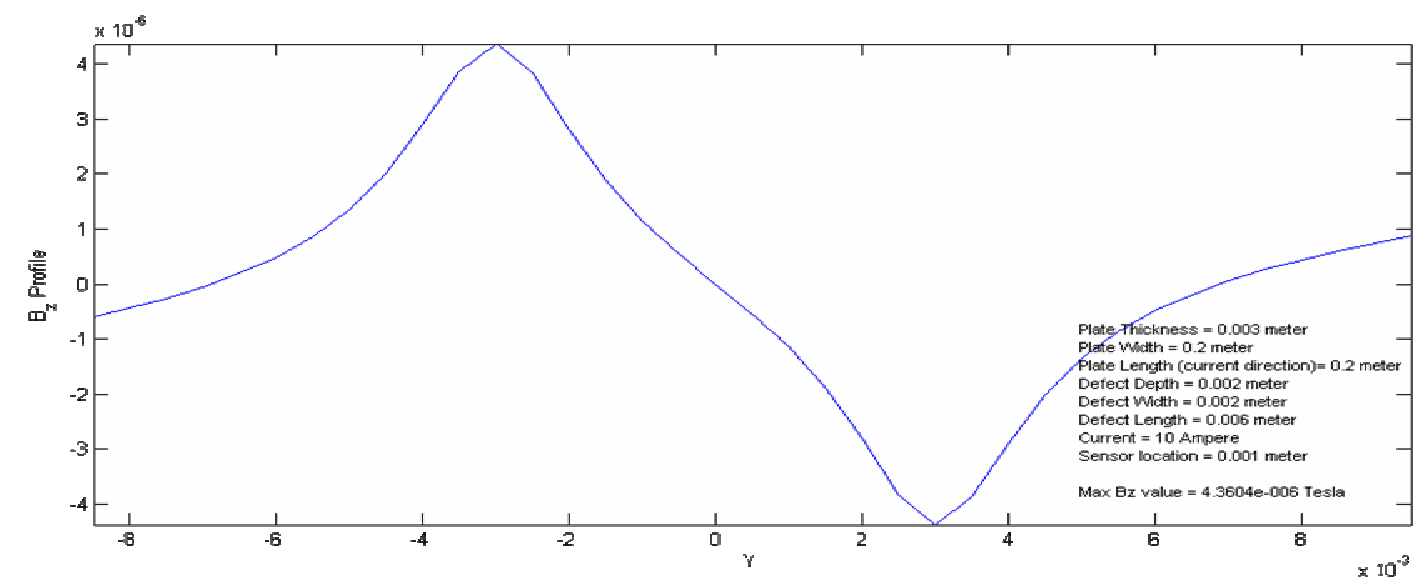

( b )

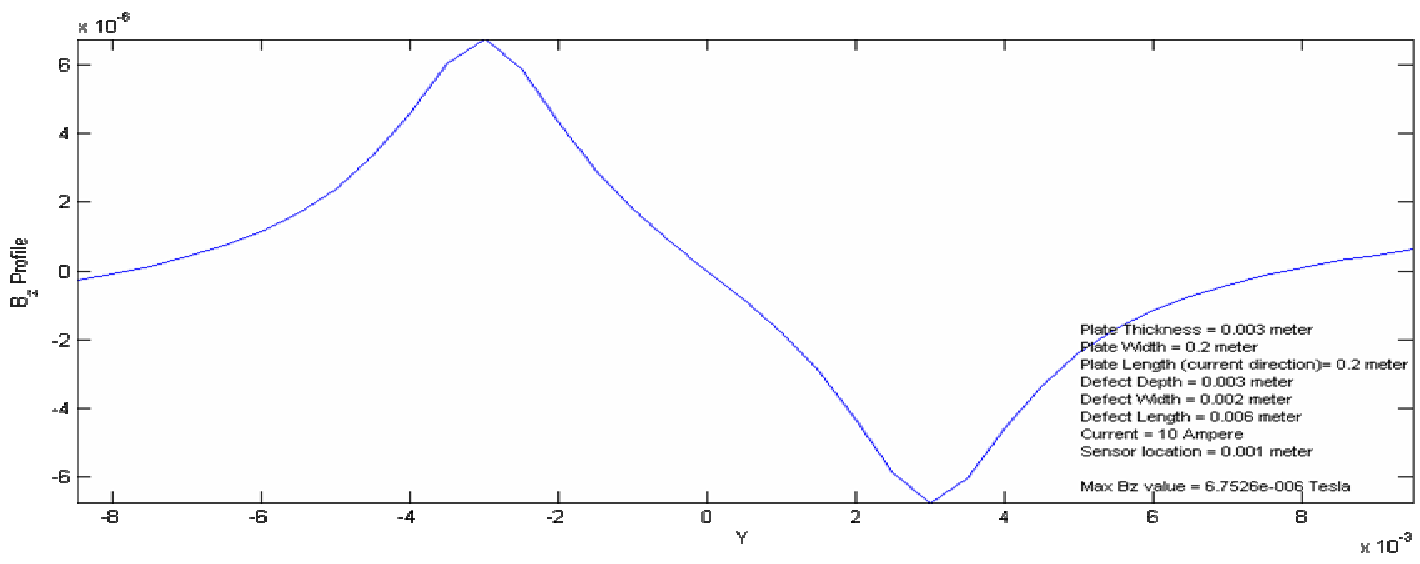

(c)

Figure 33 Crack depth factor. Where, in ( a ) tc $=0.001 \mathrm{~m}$,

( b ) tc $=0.002 \mathrm{~m}$, and in ( c ) tc $=0.003 \mathrm{~m}$-thru crack-. 


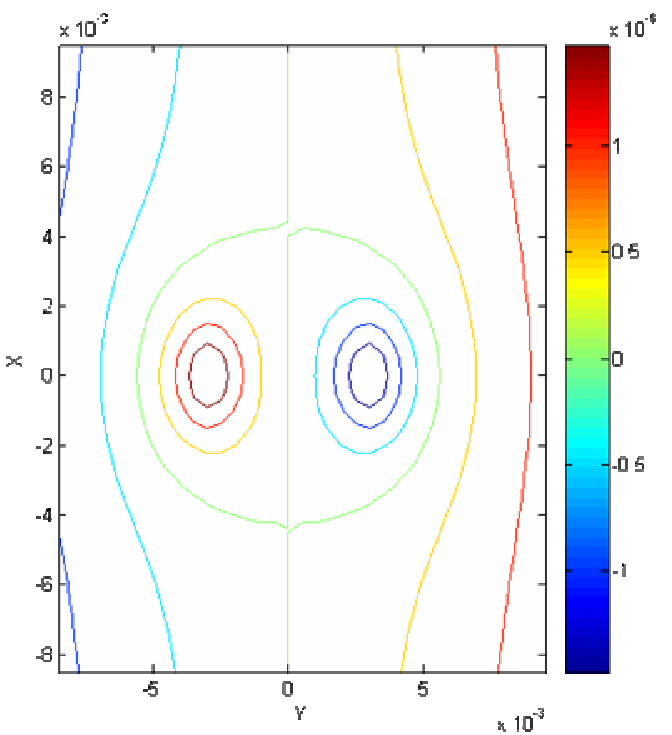

( a )

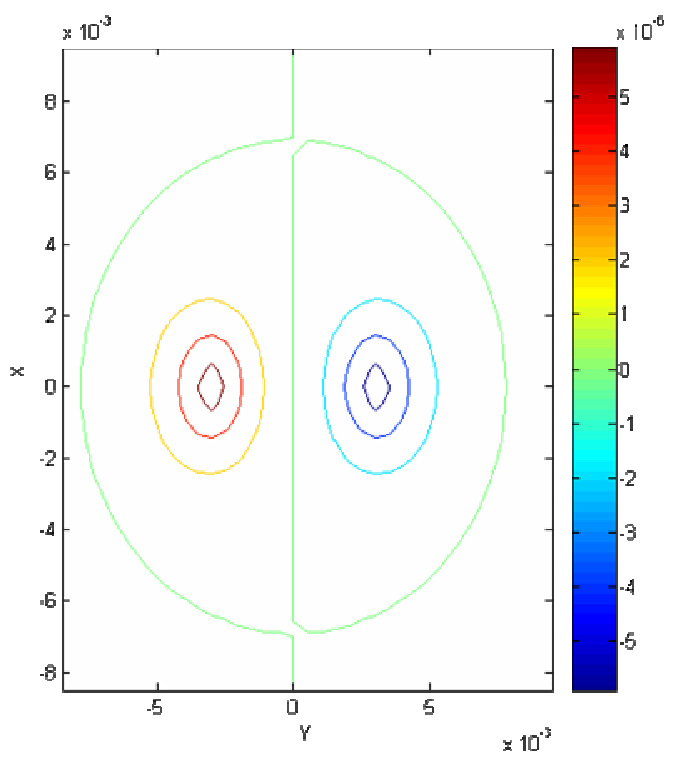

( b )

Figure 34 Magnetic field contour lines around the crack location. Where, in ( a ) tc $=0.001 \mathrm{~m}$, and $(\mathrm{b}$ ) tc $=0.003 \mathrm{~m}-$ thru crack-.

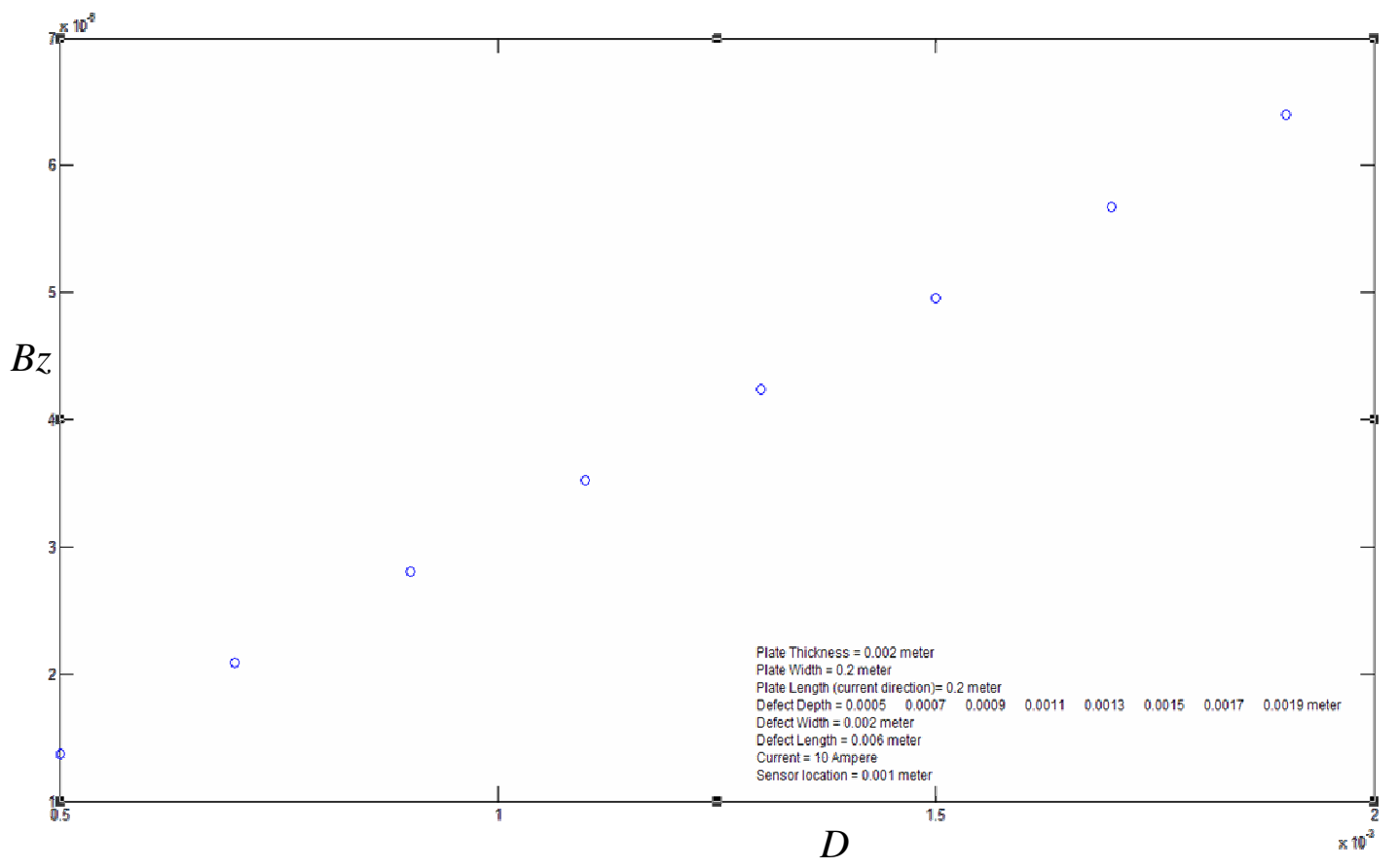

Figure 35 Crack depth effect on the magnetic field at the crack tip 


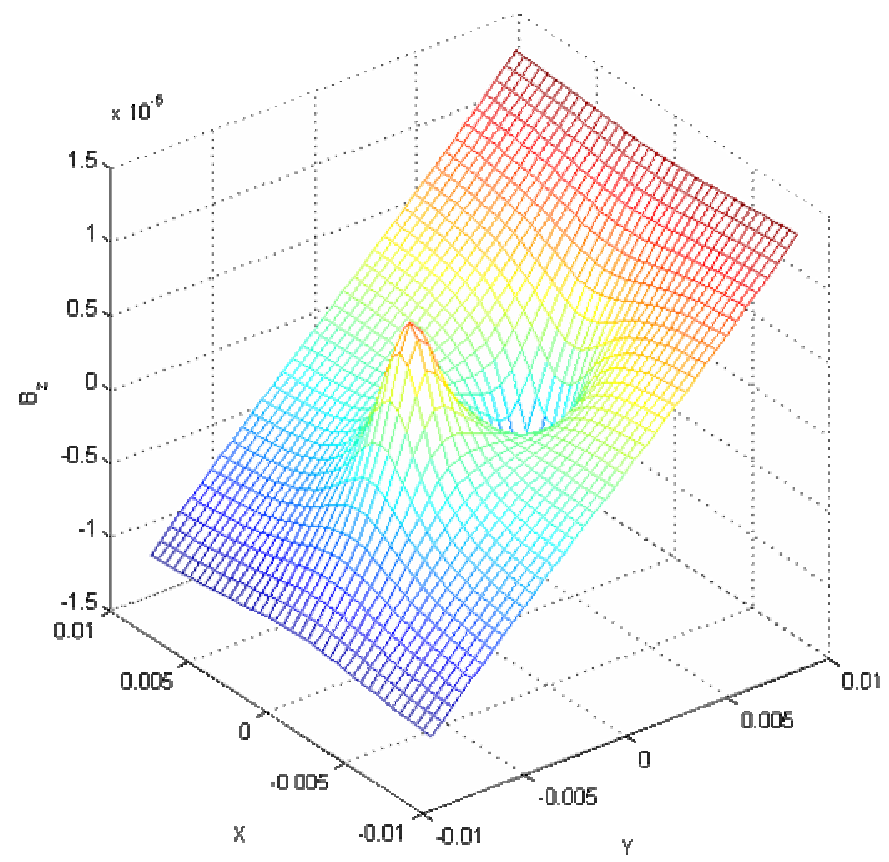

Figure 36 Magnetic field around the crack with a $0.0005 \mathrm{~m}$ depth

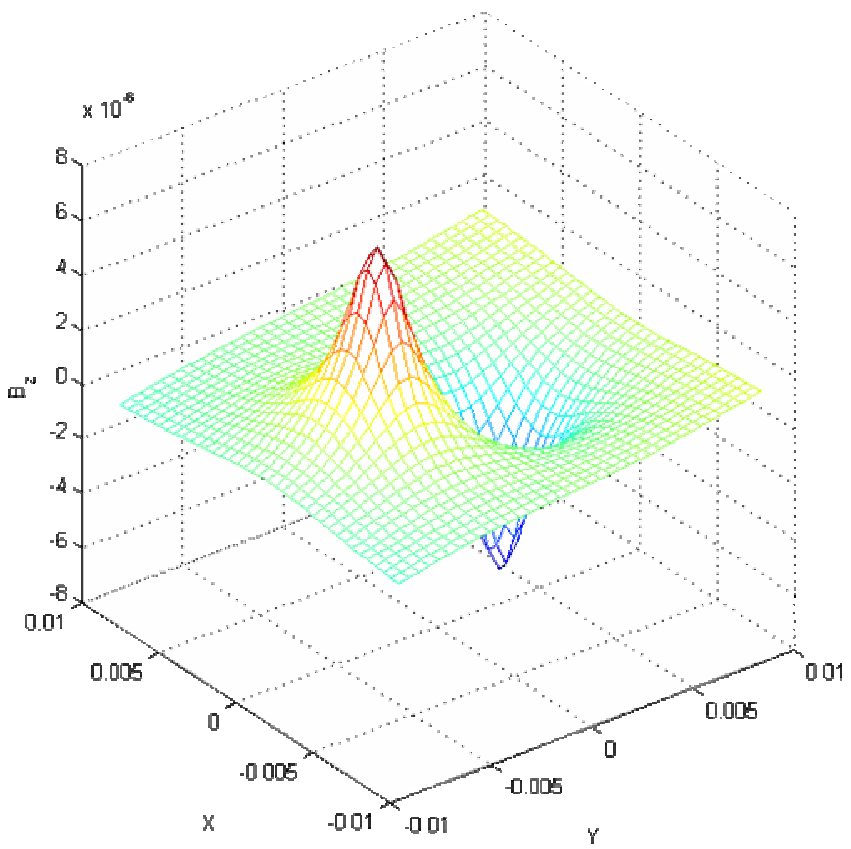

Figure 37 Magnetic field around the crack with a $0.003 \mathrm{~m}$ depth 


\subsection{Magnetic Signal Enhancement for Better Detection}

From the previous sections' results and discussion, the magnetic signal produced by the crack or defect is found to be very small compared to the magnetic field that is produced by the edges of the plate. However, this is not the only magnetic field that can interfere with our sensor readings. There is also the magnetic field produced by the wires that carry the current, and how they are connected to the plate under testing. This section will give some discussion and develop ways to eliminate or minimize the unwanted magnetic fields.

\subsubsection{Magnetic Fields Produced by the Wires}

First, note that the interest of this work is the normal component of the magnetic field above the plate. Any other magnetic field that is not interfering with the sensor reading will not affect the test. There are two ways to connect the wires to the plate; either parallel or normal to the plate surface. Figure 38 shows the magnetic fields around the plate when the wires where connected in parallel to the plate, and Figure 39 shows the magnetic fields around the plate when the wires where connected normal to the plate. There should be no movement in the wires while running the test, because any small movement will cause complex magnetic field around the plate.

In Figure 38 ( $a$ and $b$ ), the wires where connected in parallel to the plate surface and therefore the magnetic fields produced by the wires have a normal component and they will interfere with the readings. Therefore, it is better to connect the wires to the plate as in Figure 38 ( $a$ and $b$ ), where the wires are connected normal to the surface of the plate and their corresponding magnetic fields are not normal and will not interfere with the sensor readings. 


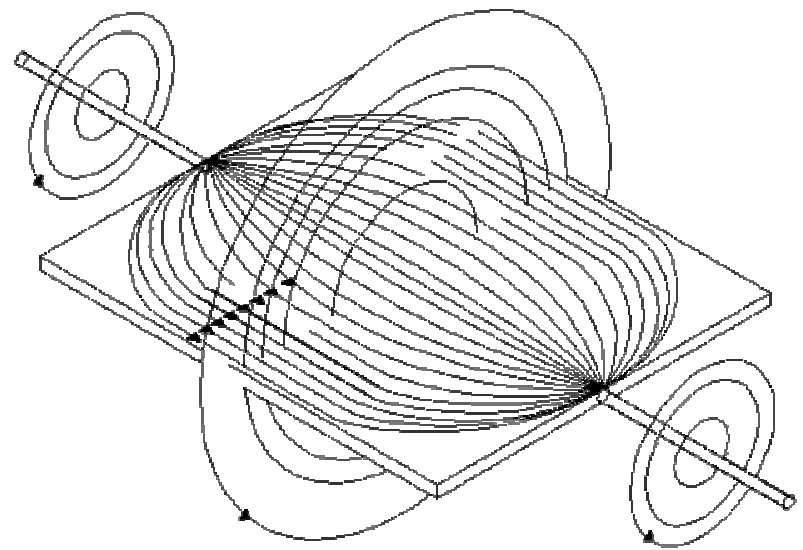

(a)

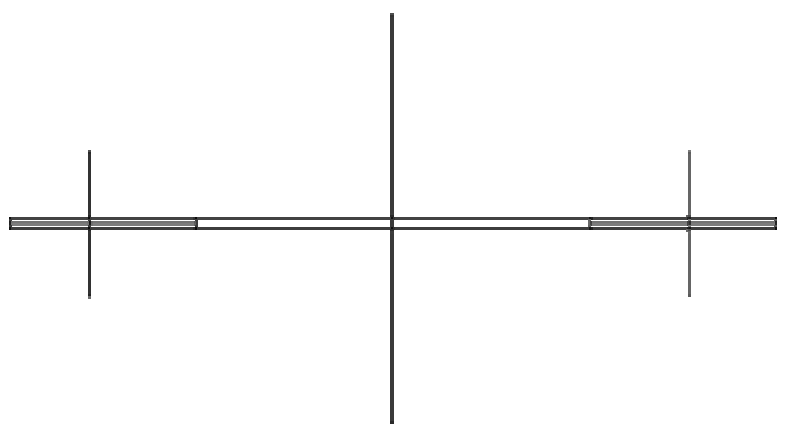

(b)

Figure 38 Magnetic fields around the plate when the wires are connected in parallel to the plate.

(a) 3D view and (b) Front view 


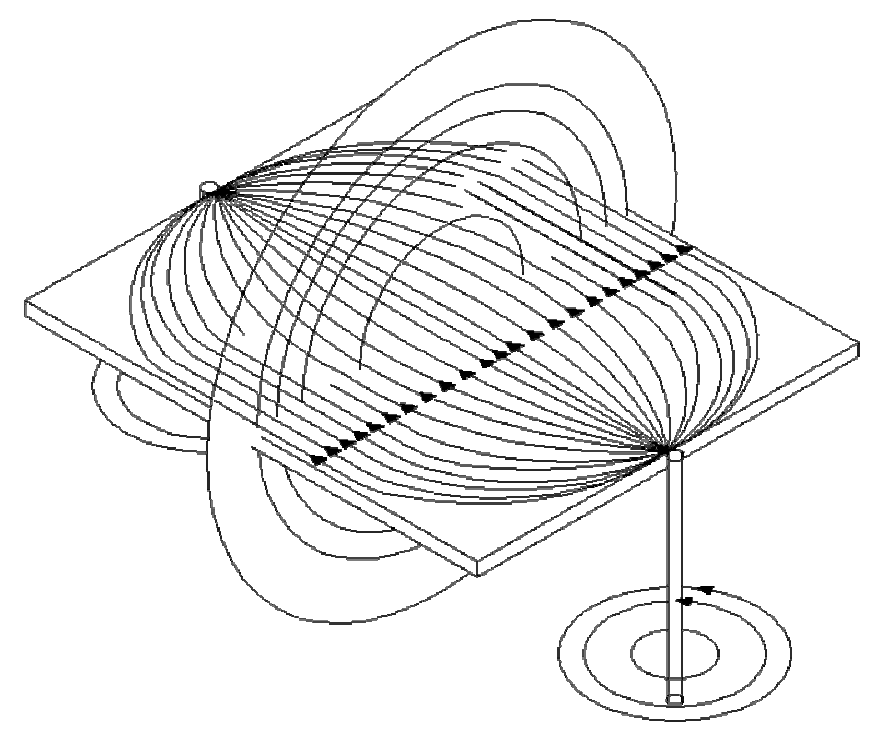

(a)

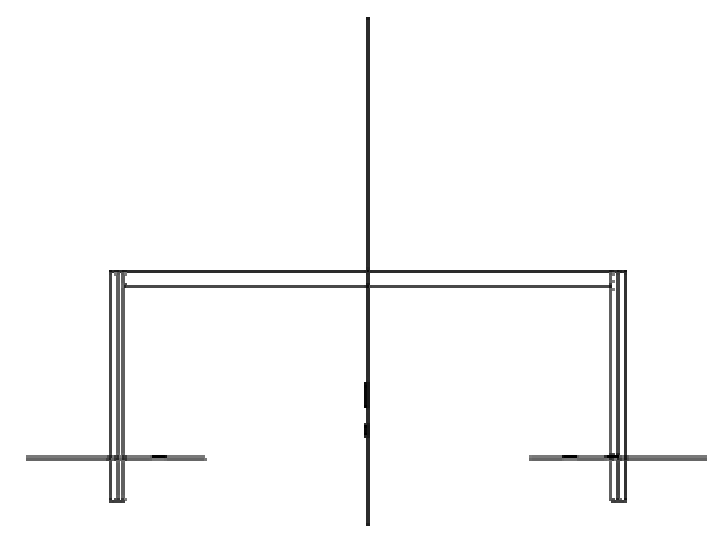

(b)

Figure 39 Magnetic fields around the plate when the wires are connected normal to the plate.

(a) 3D view and (b) front view 
Another issue related to the wires is that in this analysis it is assumed that the current is uniform all over the plate, Figure 40. But, in fact, if the wires are connected in the same way as suggested in Figure 39, the current is not uniform all over the plate. It is assumed that the current a way from both edges is uniform and the analysis is valid since the area of interest is around the crack at the middle of the plate. Figure 41

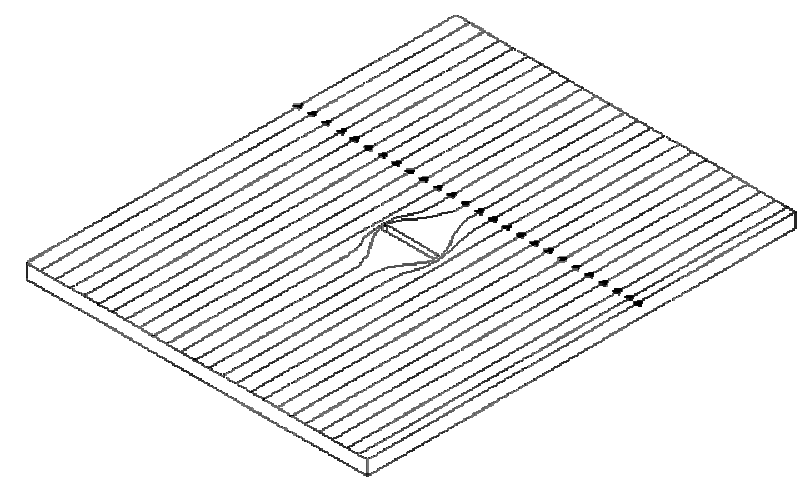

Figure 40 Uniform Current in the plate.

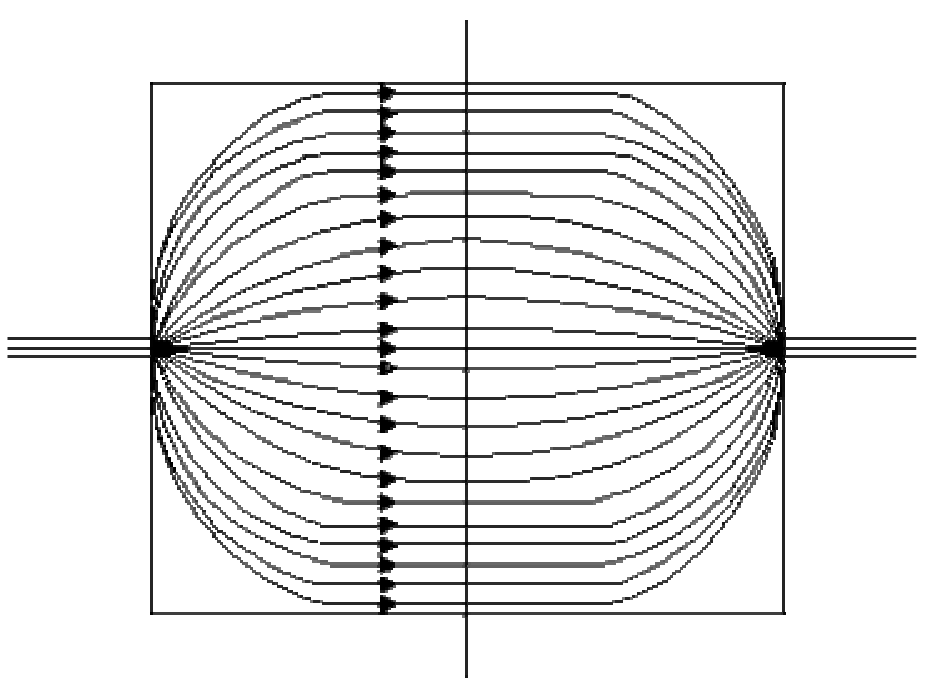

Figure 41 Current distribution according to figure (32) 


\subsubsection{Magnetic Fields Produced by the Plate Edges}

The magnetic signal produced by the crack or defect is very small compared to the magnetic field that is produced by the edges of the plate. When the crack is small, it is hard to detect the crack because the magnetic field signal associated with this crack is very weak compared to the one produced at the edges. To strengthen the crack signal, it is important to eliminate or reduce the signal due to the edges. The way to do that is by applying the concept of supper-position. If there are two wires carrying the same current intensity but in opposite directions, they will produce the same magnitude of magnetic field at a point in the middle distance between them but in the opposite direction. Thus, they will cancel each other.

Using the same idea, another plate is used, with no crack, that has the same properties as the plate under testing, with same dimensions and with the same current flowing thru it but in the opposite direction to the original current. Therefore, the magnetic field generated by the new plate, the dummy plate, will cancel the one produced by the defected plate. In real life, it is hard to completely cancel the effect of the edges since the point to be measured is not at the middle distance between the two plates, but this will minimize the effect from the edges, so the signal from the crack will be strong enough since the field from the edges will be close to zero around the crack. Figure 42 shows the illustrative diagram for both the test plate and the dummy plate and it shows the opposite magnetic field at the plate edges. The two plates are connected at one end and the dummy plate can be placed over or under the defected plate. 


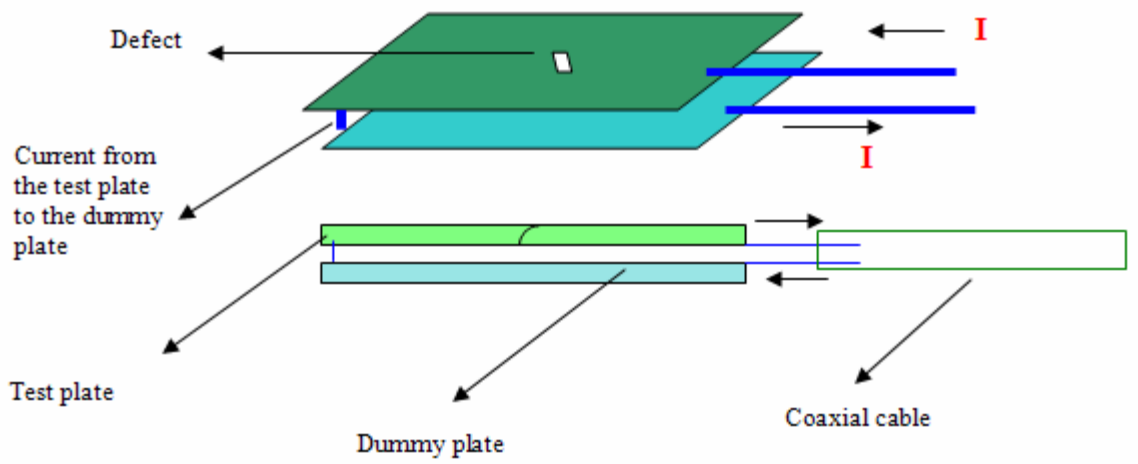

Figure 42 Illustrative diagrams for both the defected plate and the dummy plate

In the following subsections, the effect of the dummy plate in this analysis is discussed. Assuming that the distance between the two plates is $t_{e}$, then it is included in the analysis and repeated in the previous analysis.

\subsubsection{Effect of $t_{e}$ on the New Magnetic Field}

From the previous discussion, it is clear that when distance between the two plates is increased the effect of the dummy plate will decrease and then the magnetic field of the signal at the crack tip will fade again, Figure 43 shows this effect.

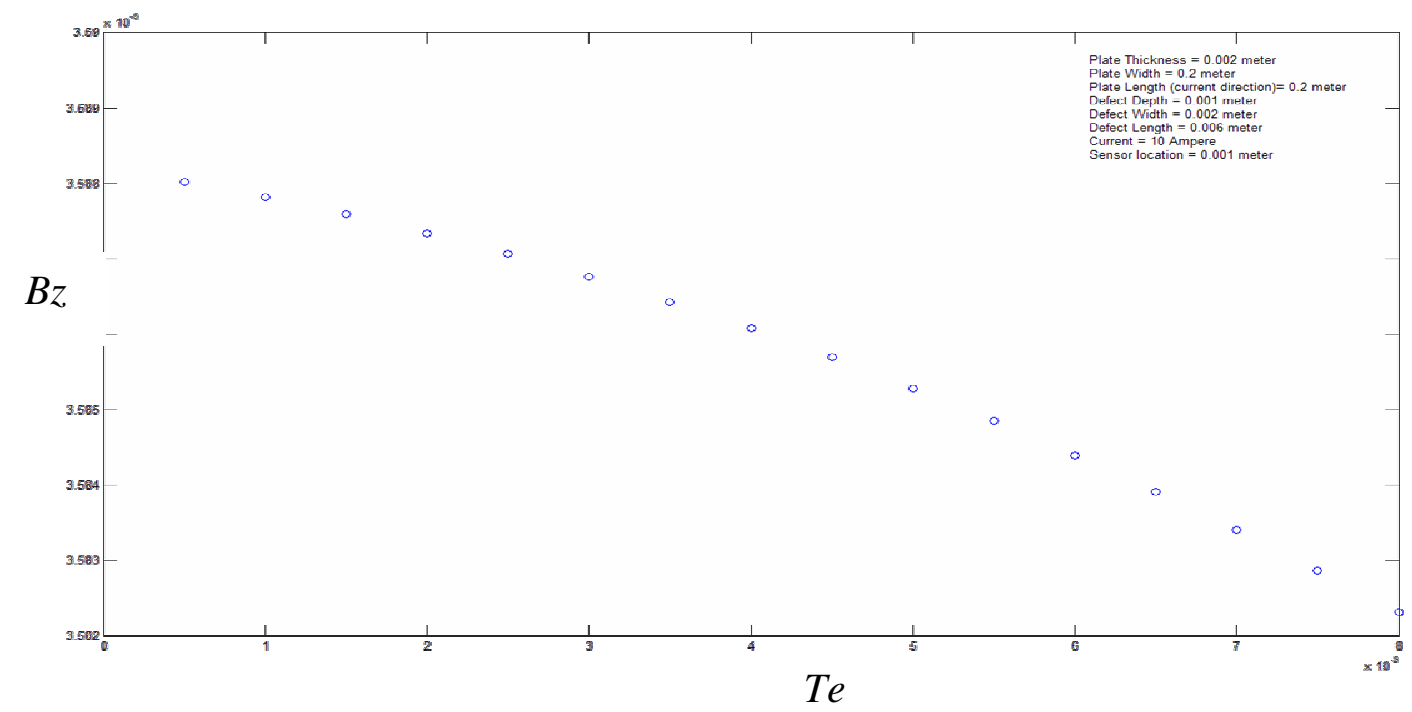

Figure 43 Effect of $t_{e}$ on the new magnetic field. 


\subsubsection{New Analysis after Minimizing the Effect of Plate Edges}

In this section, the analysis is performed using the dummy plate to see the big change in the strength on the crack signal.

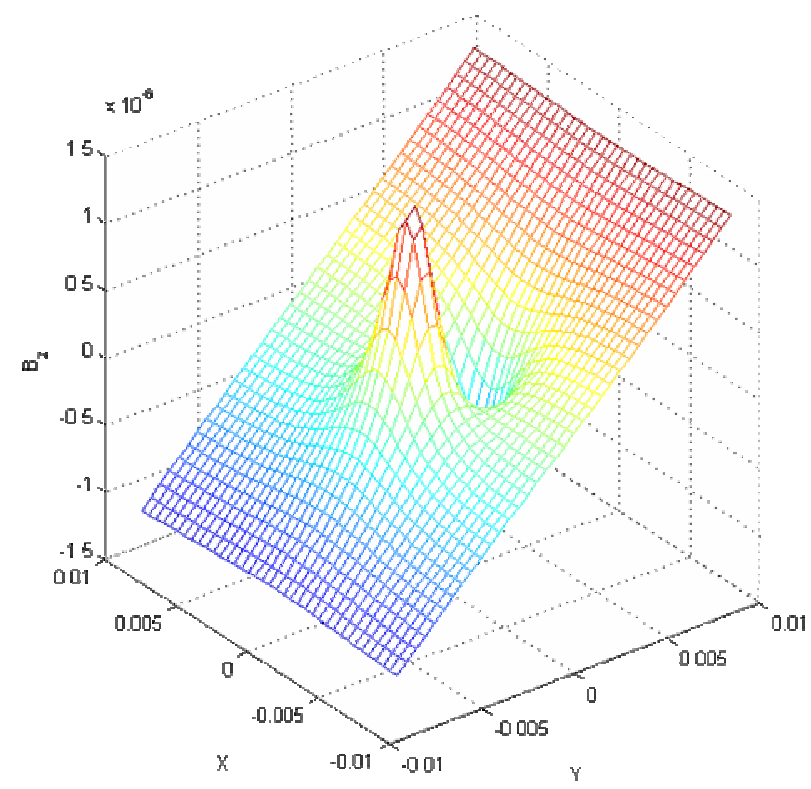

(a)

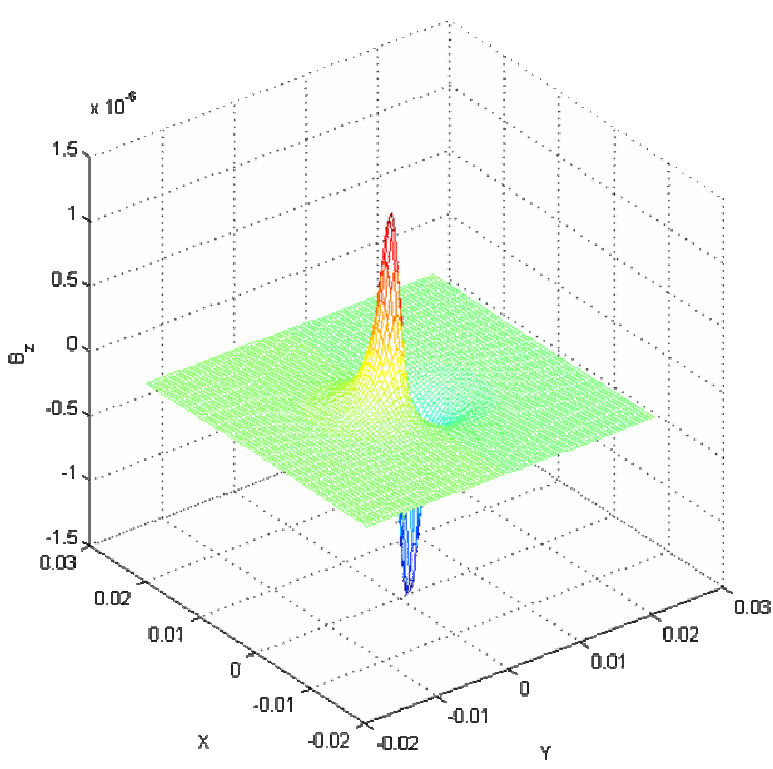

(b)

Figure 44 3D Magnetic Filed around the same Crack. (a) Without the D. plate. ( b) using the D. plate. 


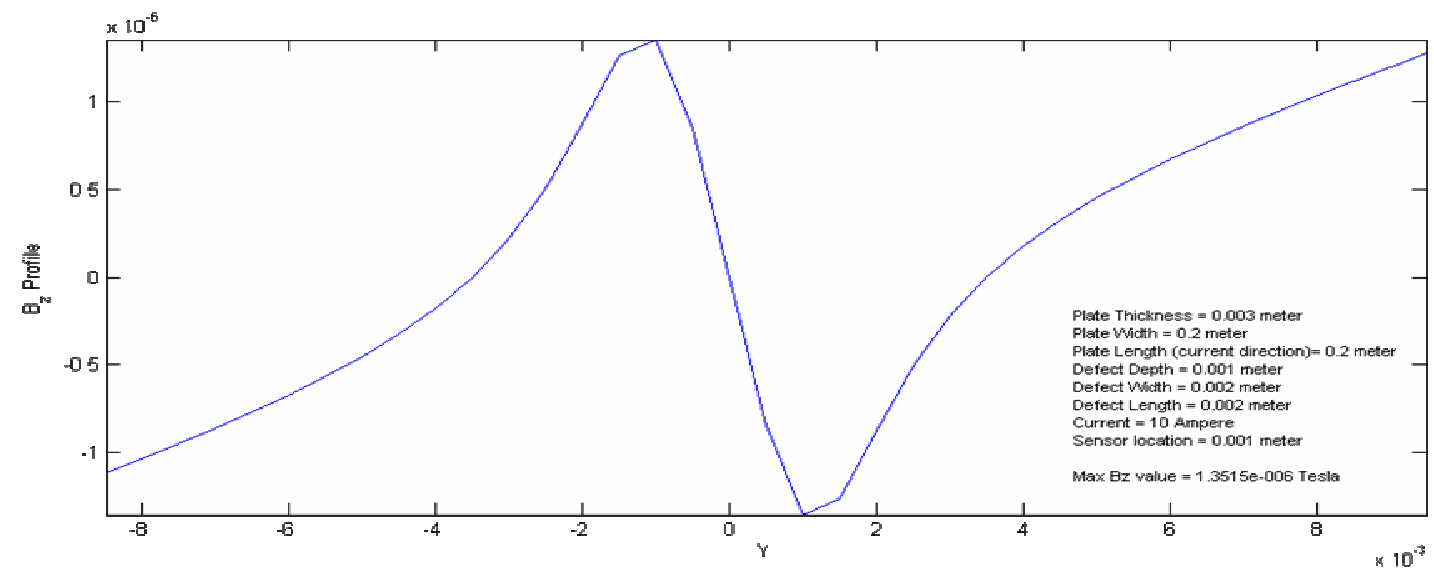

(a)

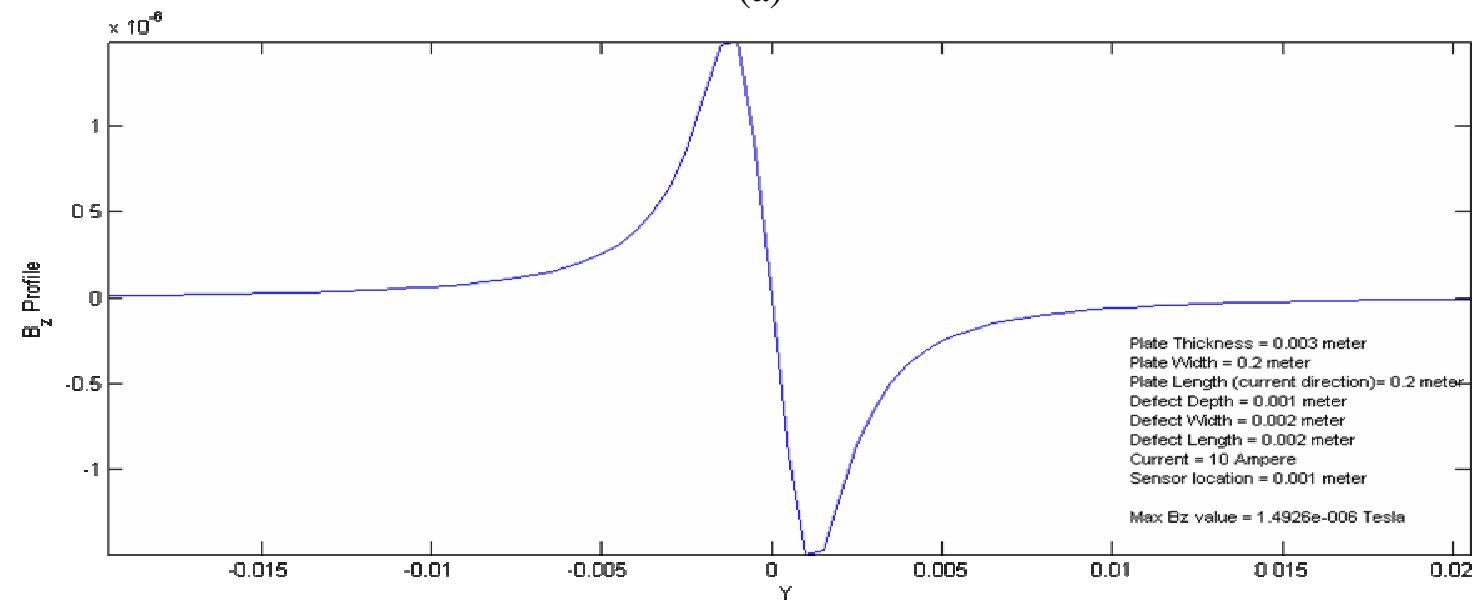

(b)

Figure 45 Magnetic signal profile around same the crack. (a) Without the D. plate. ( b) using the D. plate.

Figure 44 and Figure 45 show the change in the magnetic field after using the minimization of the edges' effect. It is clear that the magnetic filed around the crack is close to zero which makes it easy for the sensor to not only detect the crack but also to detect the tip of crack since the magnetic field around the crack is close to zero and there is only variation in the field due to the crack.

When the crack depth is very small, it is hard to detect the crack as was noticed in the previous chapters. In Figure 46 and Figure 47, the change in the shape and magnitude of the crack profile is observed for a crack with a $0.0005 \mathrm{~m}$ in depth due to the use of the dummy plate (D. plate). 
To see the effect of the crack dimensions on the magnetic field at the tip of crack, the same analysis is carried out. The big improvement in the signal strength can be seen in Figure 48, Figure 49, and Figure 50.

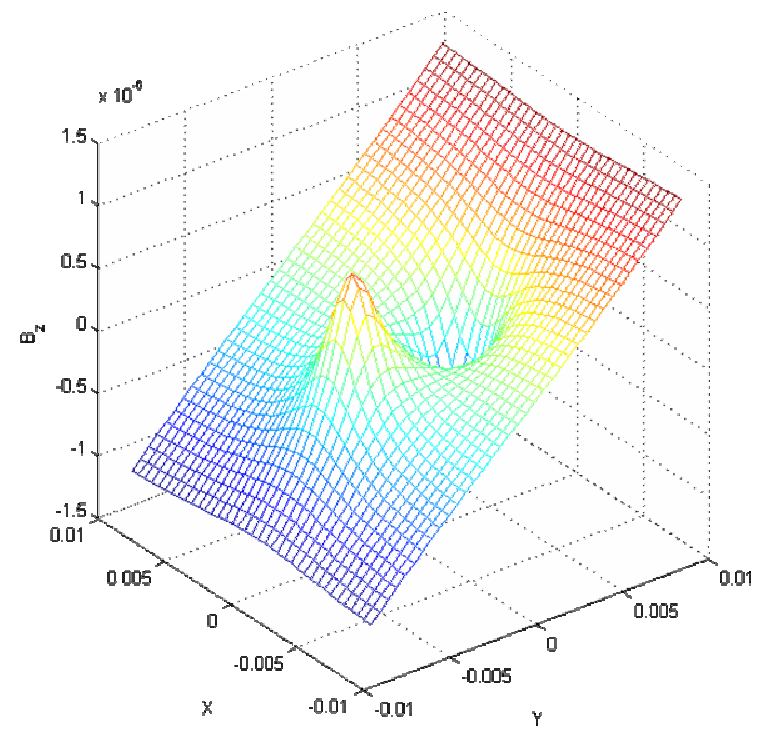

(a)

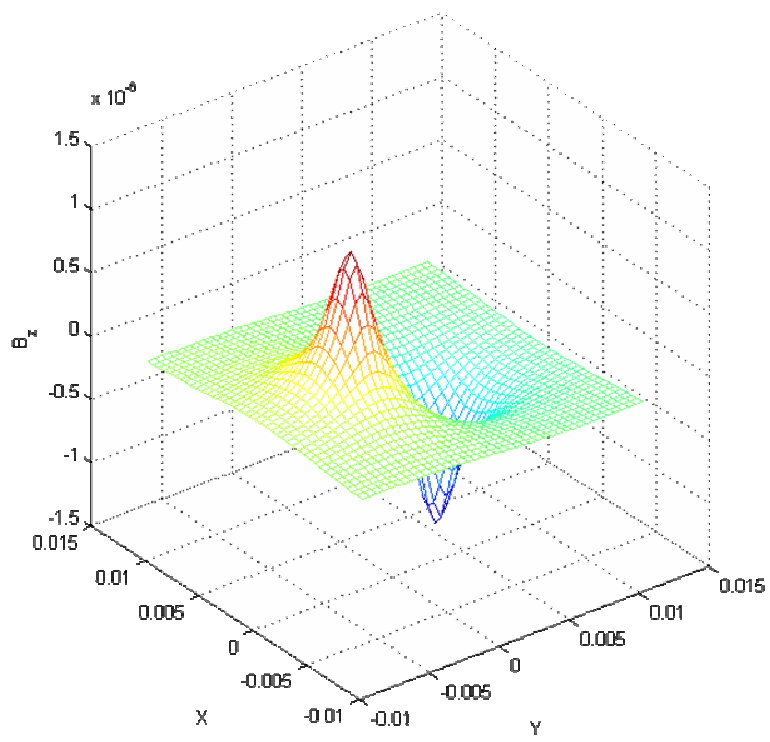

(b)

Figure 46 3D Magnetic field for crack with $0.0005 \mathrm{~m}$ depth. Where, (a) without D. plate (b) with D. plate 


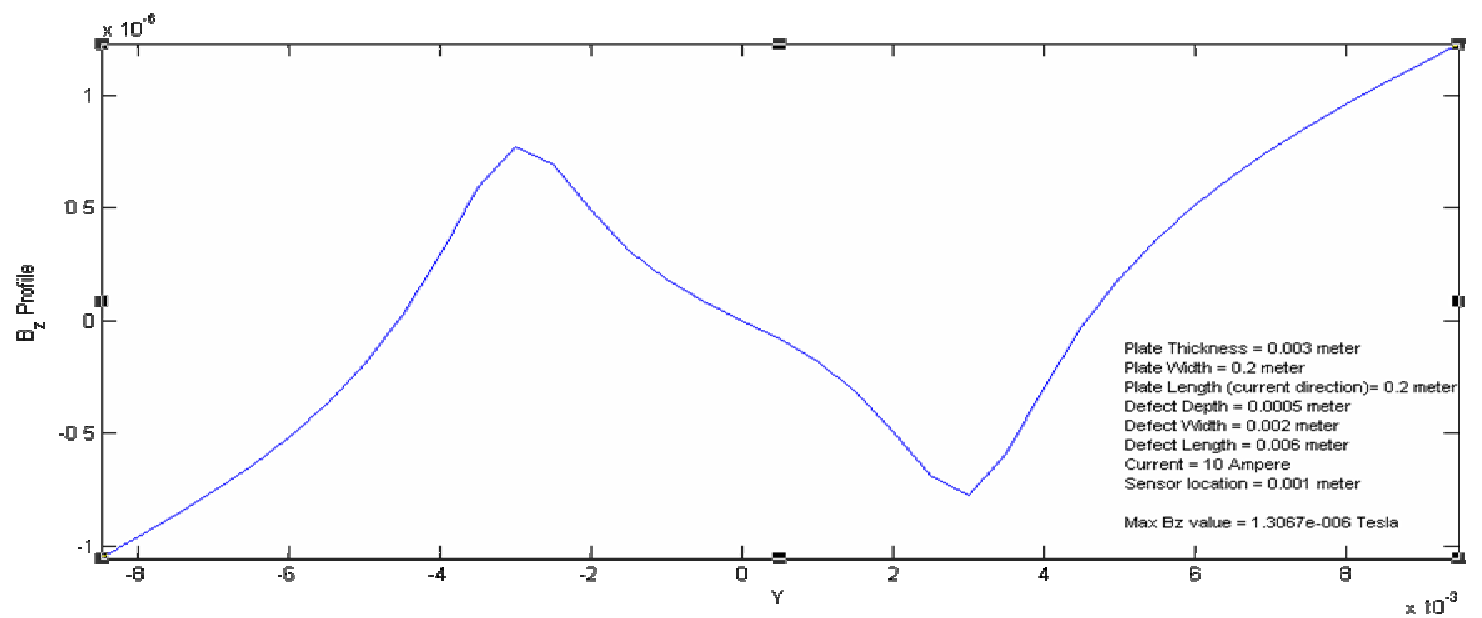

(a)

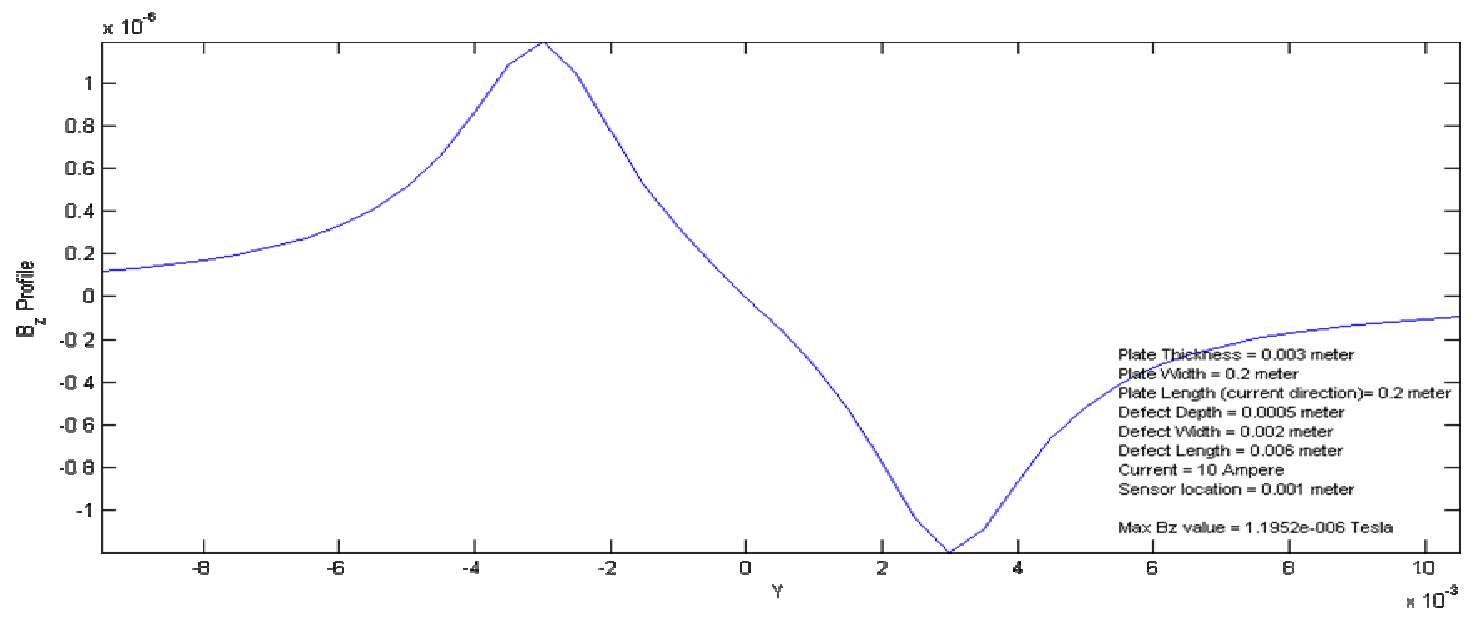

(b)

Figure 47 Magnetic signal profile around a crack with $0.0005 \mathrm{~m}$ depth. Where, (a) without D. plate (b) with D. plate

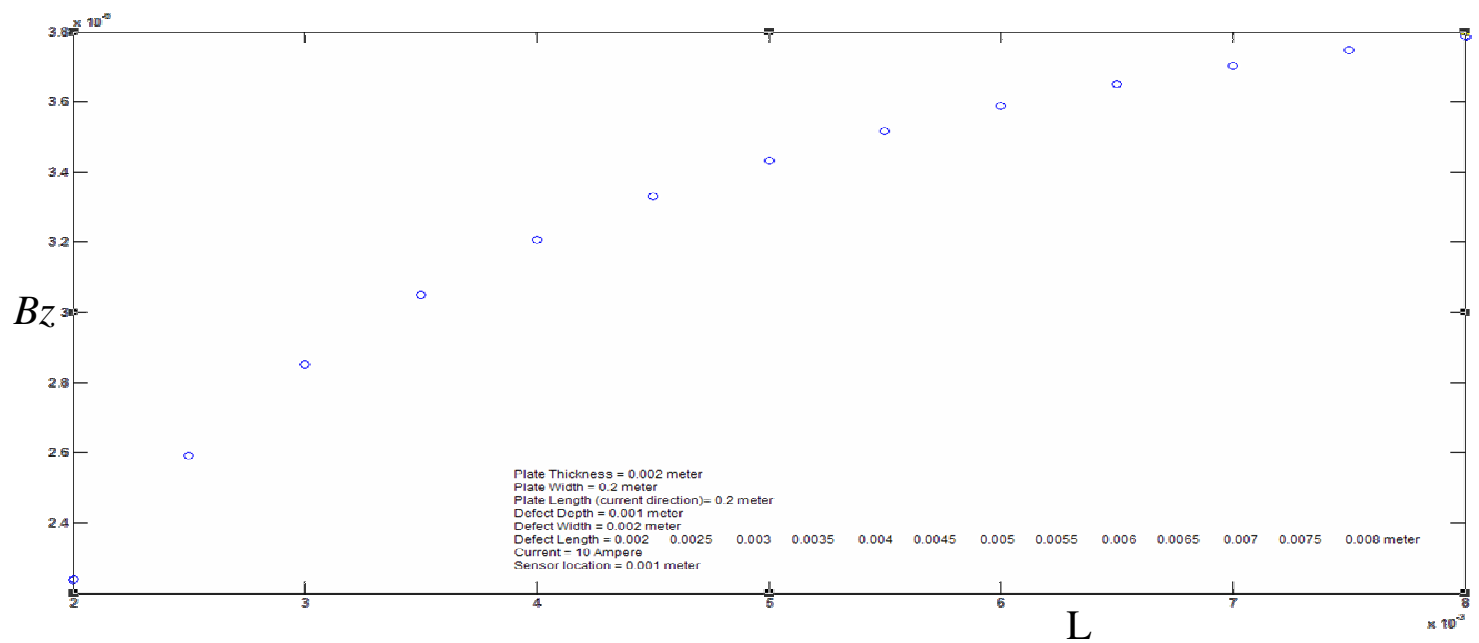

Figure 48 Crack length effect on the magnetic field at the crack tip with using the D. plate 


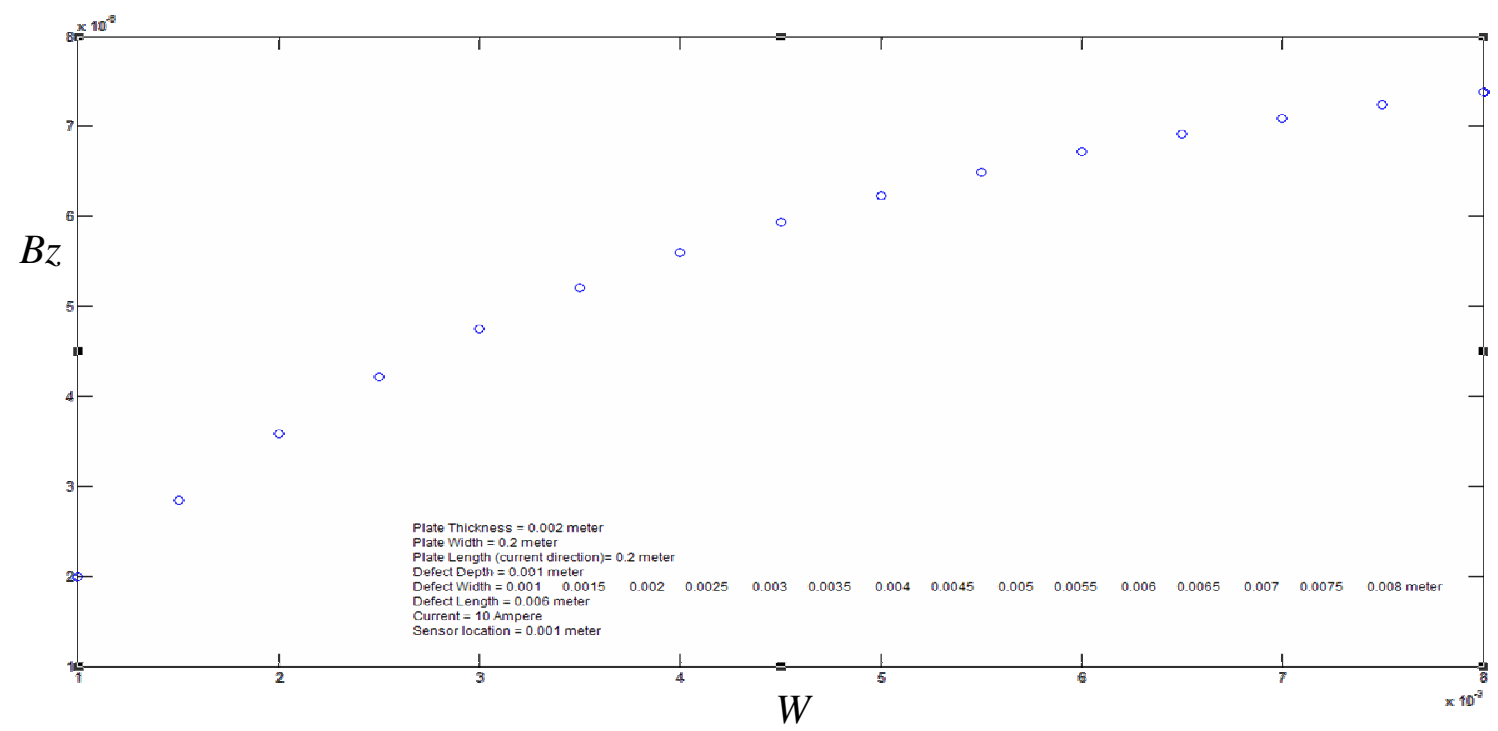

Figure 49 Crack width effect on the magnetic field at the crack tip with using a D. plate

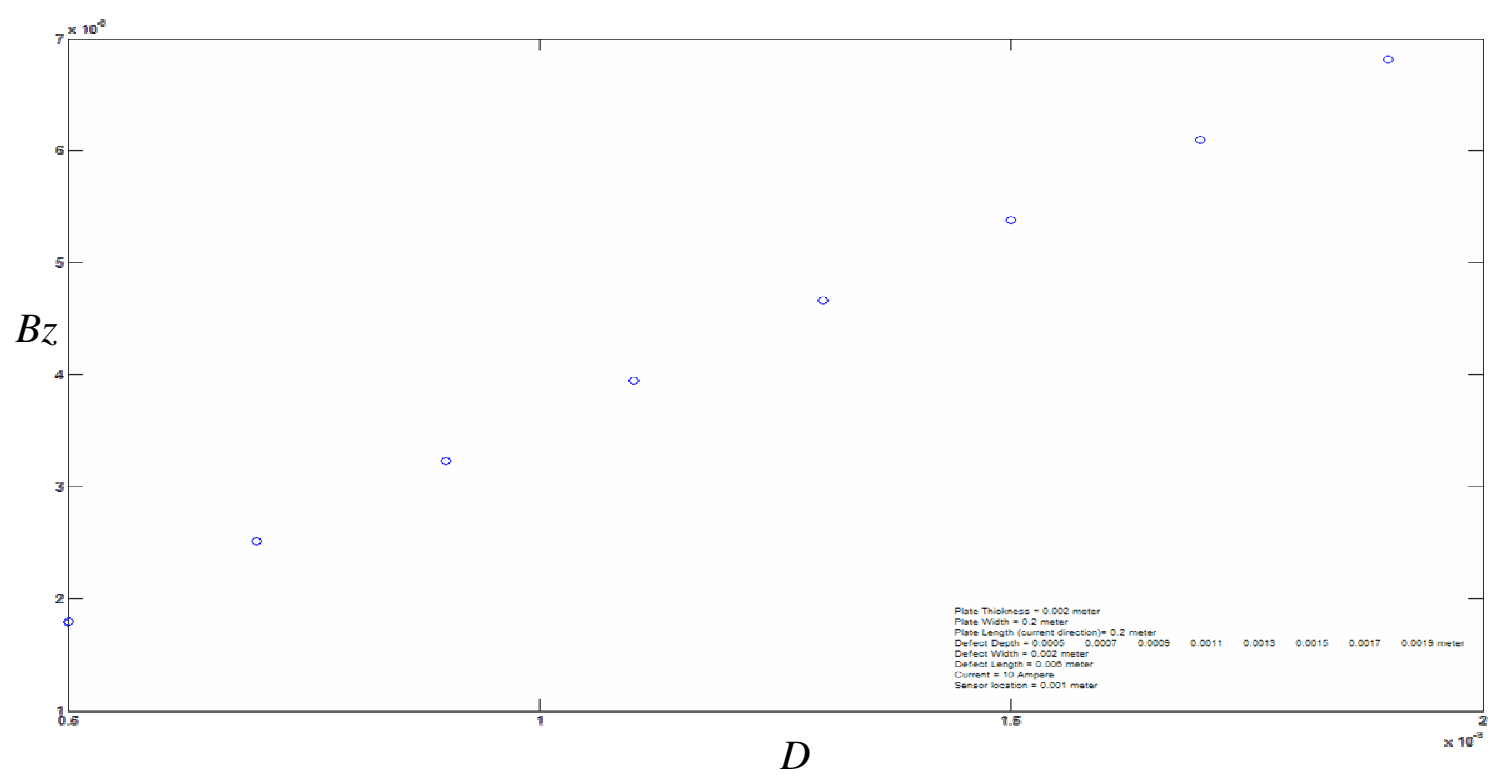

Figure 50 Crack depth effect on the magnetic field at the crack tip with using a D. plate

In summary, changing the crack profile will change the magnetic field around the crack and at the crack tip. Using another plate with opposite current direction can minimize the magnetic field produced by the plate edges. The effect of the new plate is in the magnitude and shape of the signal. Increasing the signal strength helps the sensor to detect the crack and the clear shape helps to locate the crack tips. 


\subsection{Method Capability}

In this section, the method capability is discussed and tested to detect small cracks in non-ferromagnetic structures. Detection of crack locations and tips or to be able to generate enough data for the neural network modeling depends largely on the sensor sensitivity. Sensor technology provides very sensitive sensors that can scan magnetic fields in a $\mu \mathrm{G}$ range.

The same analysis is carried out to simulate the magnetic fields produced by very small cracks and to see if this method can give enough information of the crack signature in the output signal. This analysis will run on models that are not using the dummy plate design.

\begin{tabular}{|r|c|c|c|c|c|}
\hline $\begin{array}{c}\text { Current } \\
\mathrm{Am}\end{array}$ & $\begin{array}{c}\text { Sensor location } \\
\mathrm{mm}\end{array}$ & $\begin{array}{c}\text { Plate } \\
\text { thickness } / \mathrm{mm}\end{array}$ & $\begin{array}{c}\text { Crack } \\
\text { length } / \mathrm{mm}\end{array}$ & $\begin{array}{c}\text { Crack } \\
\text { width } / \mathrm{mm}\end{array}$ & $\begin{array}{c}\text { crack } \\
\text { depth/mm }\end{array}$ \\
\hline 5 & 0.0001 & 0.000635 & 0.00001 & 0.000001 & 0.000635 \\
\hline 5 & 0.0001 & 0.000635 & 0.00001 & 0.00001 & 0.000635 \\
\hline 5 & 0.0001 & 0.000635 & 0.0001 & 0.000001 & 0.000635 \\
\hline 5 & 0.0001 & 0.000635 & 0.0001 & 0.00001 & 0.000635 \\
\hline 5 & 0.0001 & 0.000635 & 0.001 & 0.000001 & 0.000635 \\
\hline 5 & 0.0001 & 0.000635 & 0.001 & 0.00001 & 0.000635 \\
\hline 5 & 0.0001 & 0.000635 & 0.001 & 0.0001 & 0.000635 \\
\hline 5 & 0.0001 & 0.000635 & 0.000001 & 0.000001 & 0.000635 \\
\hline 5 & 0.0001 & 0.000635 & 0.0000001 & 0.0000001 & 0.000635 \\
\hline
\end{tabular}

Table 2 Crack profiles for modeling very small cracks, dimensions in $\mathbf{m m}$.

Table 2 provides crack profile dimensions that used to model very small cracks in an aluminum plate with dimensions of $(152.4 \mathrm{~mm} \times 228.6 \mathrm{~mm} \times 0.635 \mathrm{~mm})$, all crack dimensions are in $\mathrm{mm}$.

The following figures show magnetic field modeling output for a sample profiles from Table 2. 


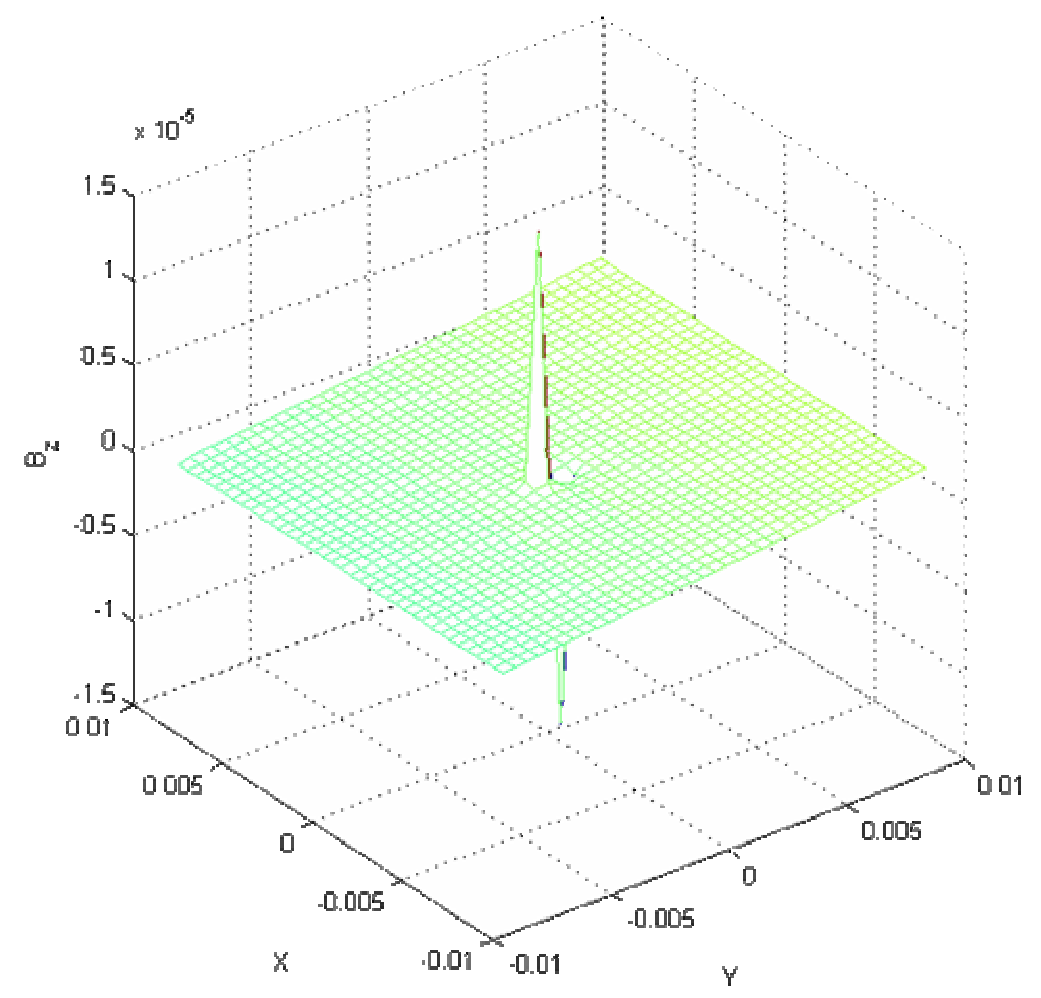

(a)

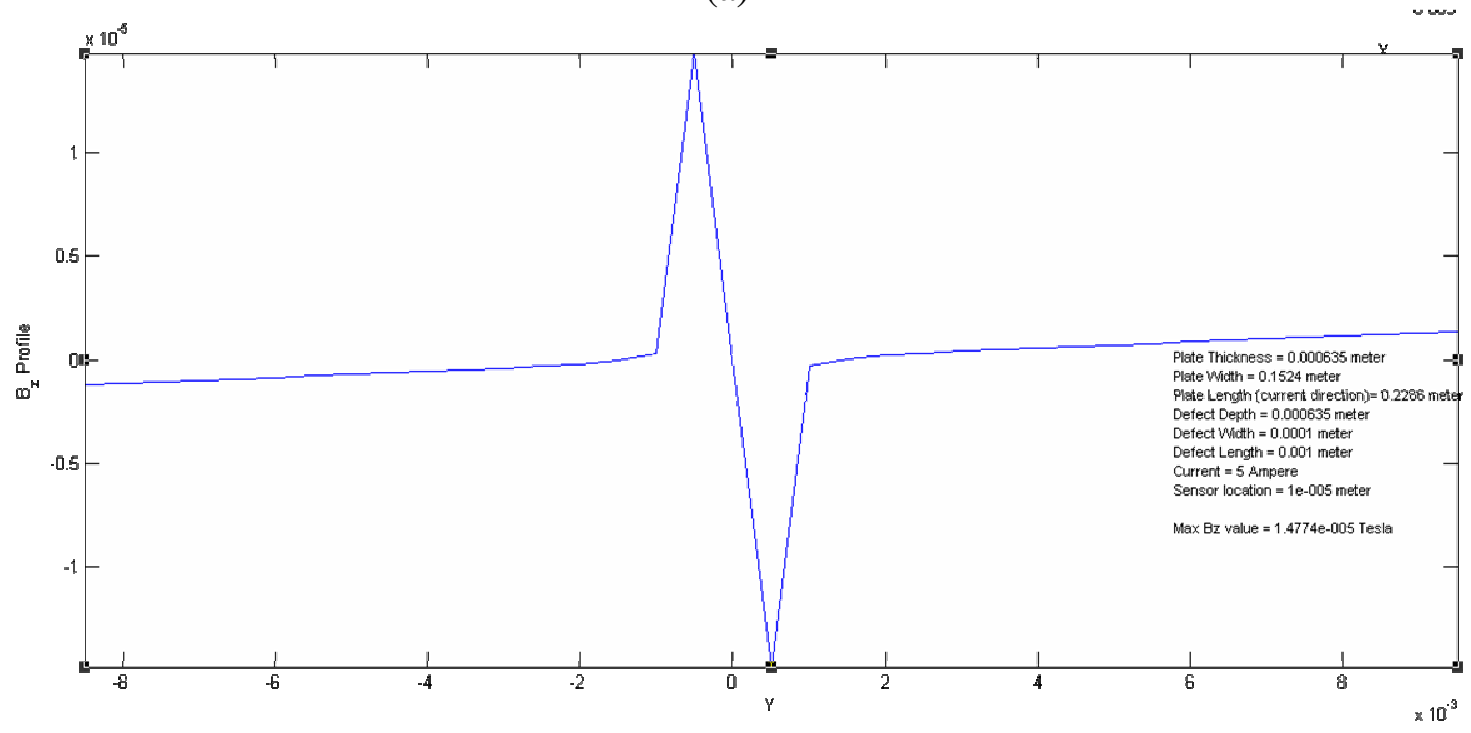

(b)

Figure 51 Magnetic field around a crack with $0.001 \mathrm{~m}$ in length and $0.0001 \mathrm{~m}$ in width. 


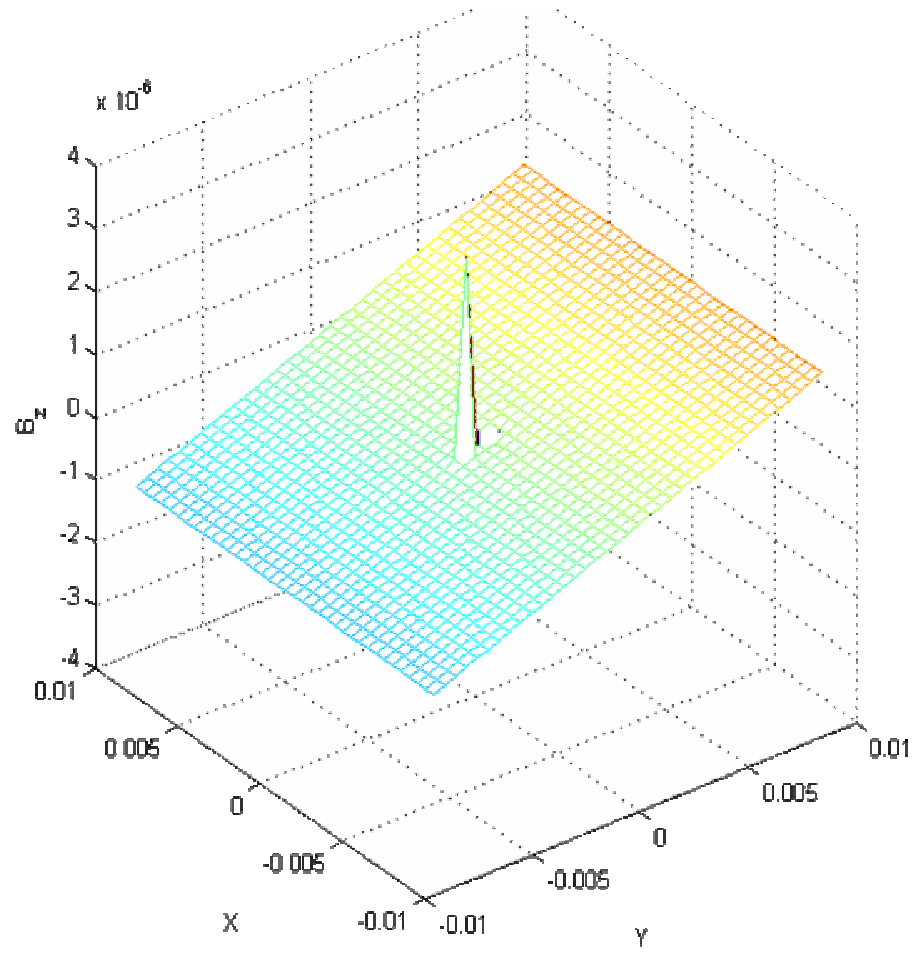

(a)

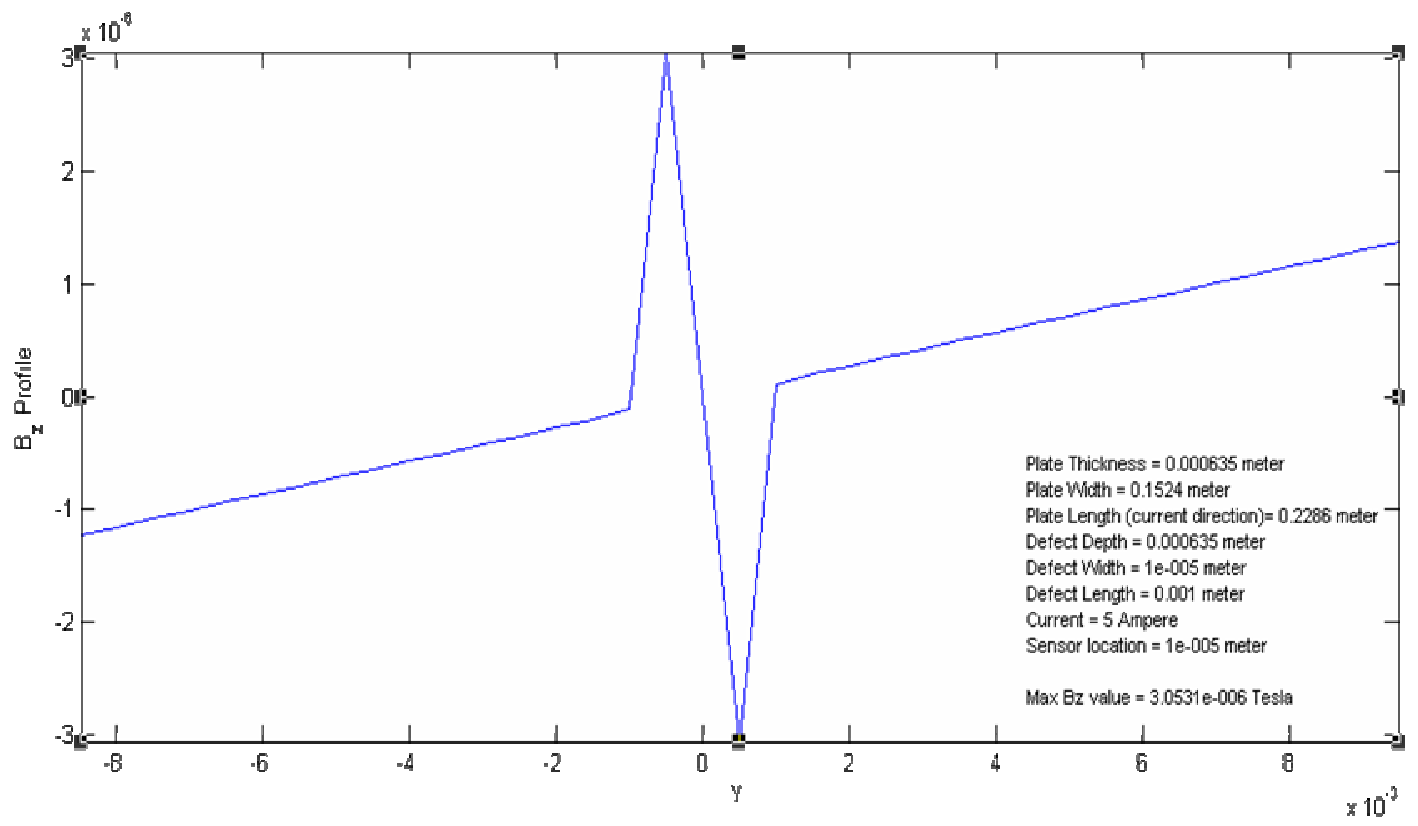

(b)

Figure 52 Magnetic field around a crack with $0.001 \mathrm{~m}$ in length and $0.00001 \mathrm{~m}$ in width. 


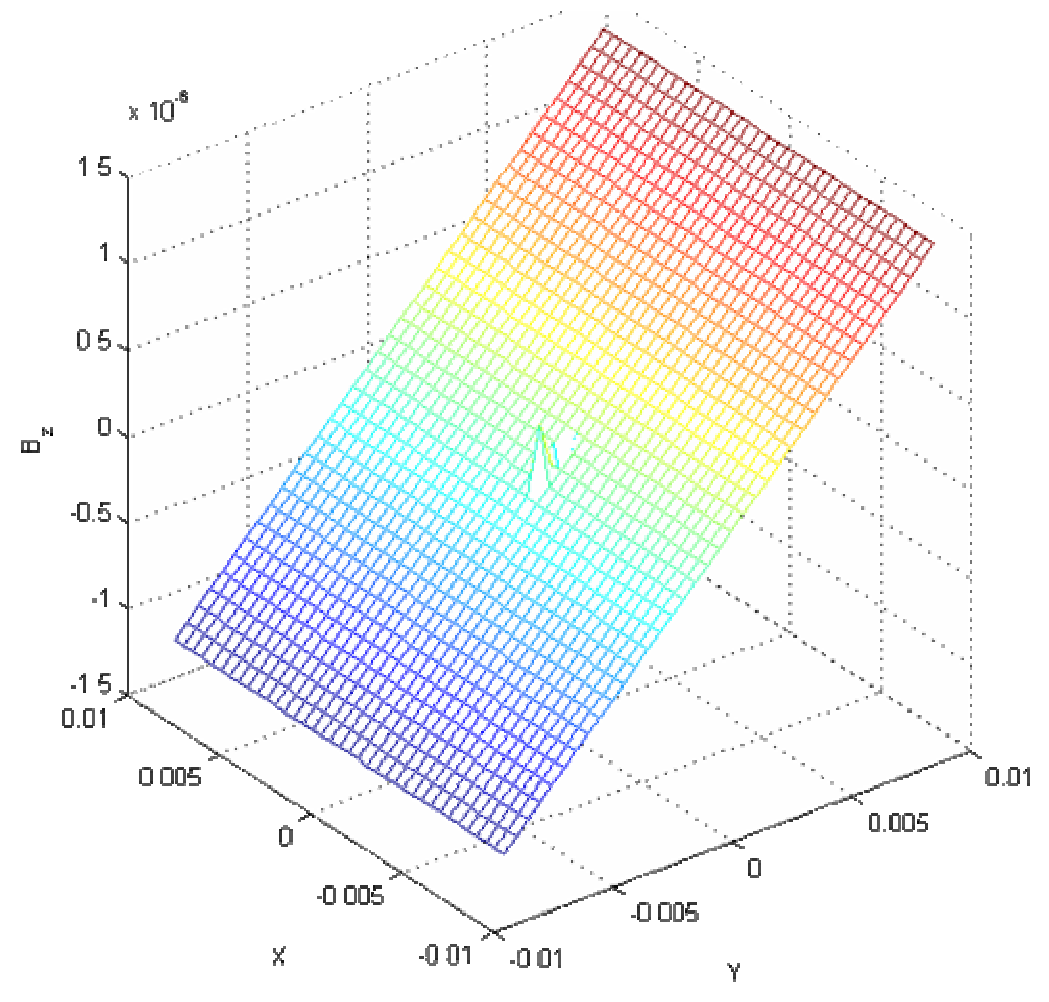

(a)

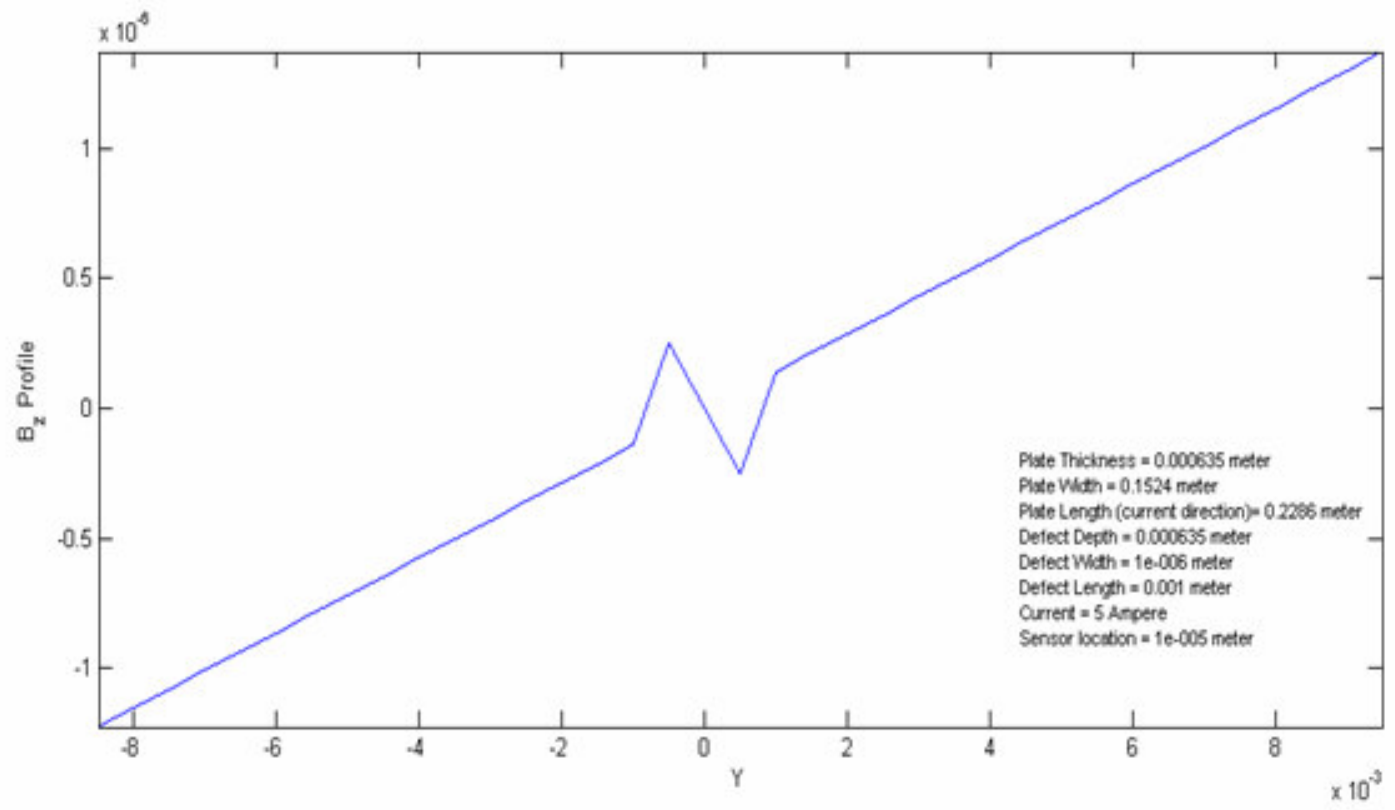

(b)

Figure 53 Magnetic field around a crack with $0.001 \mathrm{~m}$ in length and $1 \mu \mathrm{m}$ in width. 


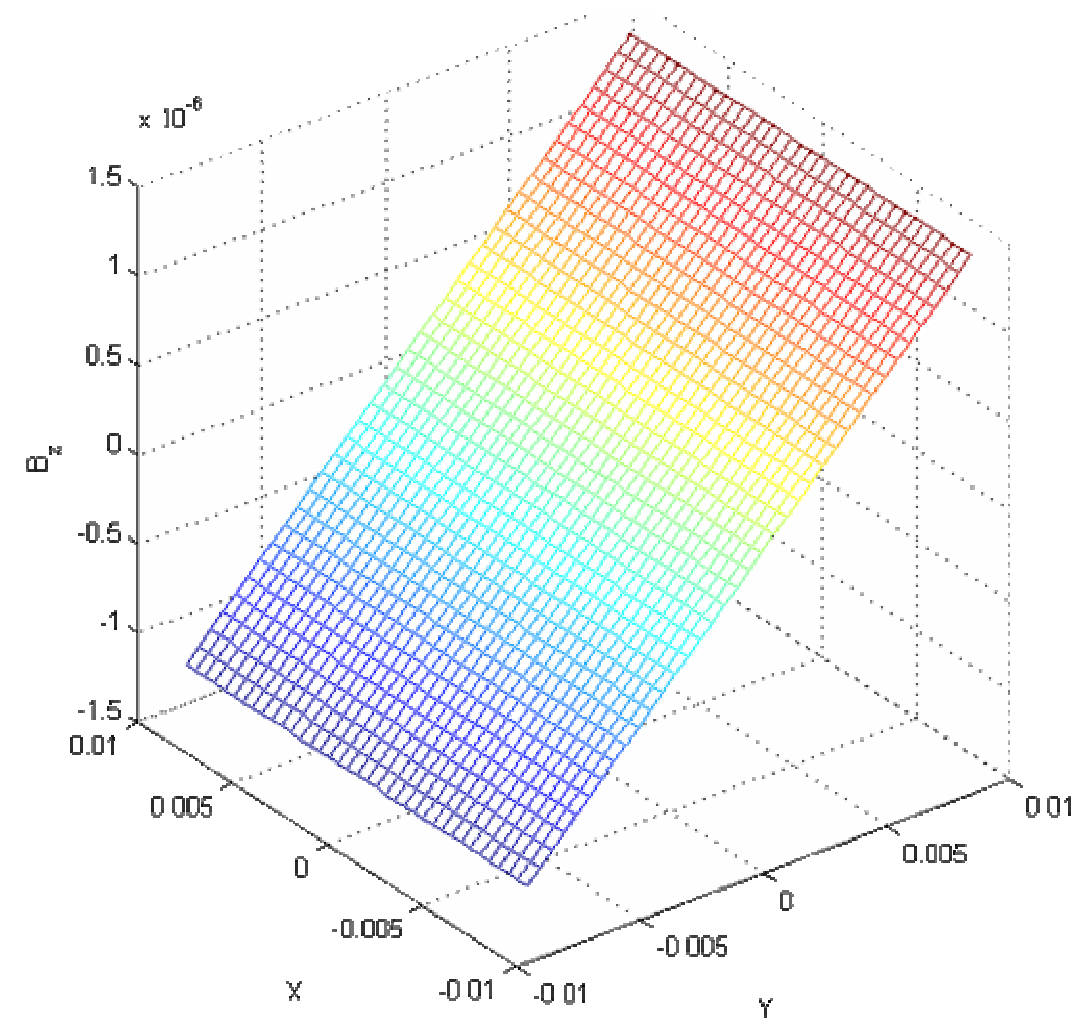

(a)

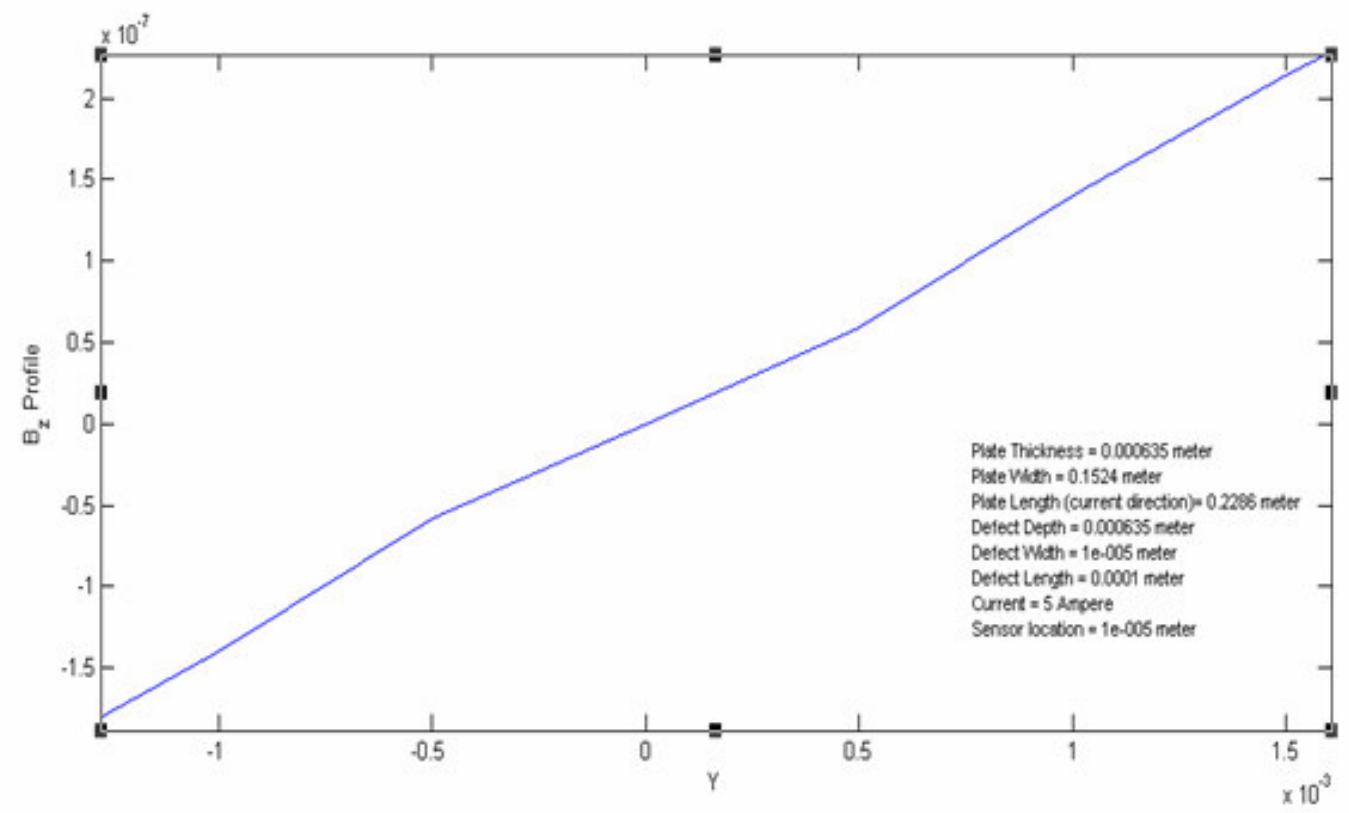

(b)

Figure 54 Magnetic field around a crack with $0.0001 \mathrm{~m}$ in length and $1 \mu \mathrm{m}$ in width. 
In Figure 51, Figure 52, and Figure 53, the magnetic field change due to the crack is clear even if the crack is very small. Decreasing the crack size will result in a small magnetic field produced by the crack at the crack tip. In Figure 53, the change is small but still the model is able to detect it. A sensitive sensor will detect this micro crack. In the case of a smaller crack than the one in Figure 53, the modeling analysis did not give enough information to detect the crack, Figure 54. In modeling, changing the element size will result in better resolution.

In summary, the method and the modeling analysis are capable of detecting small cracks in the micro-meter range. Detecting the crack in real life measurements will largely depend on the testing conditions and the senor sensitivity. 


\section{Neural Network Analysis and Solving the Inverse Problem}

Artificial Neural Network models have been studied for many years in the hope of achieving human-like performance in the fields of image recognition. These models are composed of many nonlinear computational functions operating in parallel and arranged in layers [41]. They are biologically inspired and contain a large number of simple processing elements that perform in a manner analogous to the most elementary functions of neurons. Neural networks learn by experience, generalize from previous experiences to new ones, and can make decisions. Neural elements of a human brain have a computing speed of a few milliseconds, whereas the computing speed of electronic circuits is on the order of microseconds. In spite of its very low processing speed, the human brain resolves language problems much faster than the fastest computers.

\subsection{Neural Network Modeling}

Work on artificial Neural Network models has a long history. Development of detailed mathematical models began more than 30 years ago with the work of McCulloch and Pitts, Rosenblatt, and others. More recent work by Hopfield and others, [6][38][21], has led to a new resurgence of the field. This new interest is due to the development of new net topologies and algorithms [7][13][21][19]. Recent interest is also driven by the realization that human-like performance in the areas of speech and image recognition will require enormous amounts of processing. Neural Networks provide one technique for obtaining the required processing capacity using large numbers of simple processing elements operating in parallel.

The human nervous system consists of cells called neurons. There are hundreds of billions of neurons, each connected to hundreds or thousands of other neurons. Each 
neuron is capable of receiving, processing, and transmitting electrochemical signals over neural pathways that make up the brain's communication system. Neural network models consist of a large number of neurons or simple processing units, also referred to as neurodes. An artificial neuron mimics the characteristic of the biological neuron. A set of inputs is applied, each representing an output of another neuron. Each input is multiplied by a corresponding weight, analogous to synaptic strengths. The weighted inputs are summed to determine the activation level of the neuron. The connection strength or the weights represent the knowledge in the system. Information processing takes place through the interaction among these units [38][21].

A number of considerations must be taken into account in designing a neural network model. First it must be decided what will be the structure of the model. Usually the models consist of a number of layers. The number of layers and the number of units in each layer must be chosen. They depend on the application for which the network is being designed. The most general model assumes complete interconnections between all units and handles nonconnected units by setting the corresponding connection strengths equal to zero.

A schematic model of a biological neuron is shown in Figure 55. A typical biological neuron consists of four basic parts: dendrites, the synapse, the cell body, which is also called the soma, and axon. Dendrites are branchlike structures that provide inputs to the cell body. Dendrites receive signals from neighboring neurons at connection points called synapses. The cell body essentially sums the membrane potentials provided by the dendrites. When the cumulative excitation in cell body exceeds the threshold, the cell 
fires, sending a signal down the axon to other neurons. Axons are long fibers that serve as transmission lines [39][38][21].

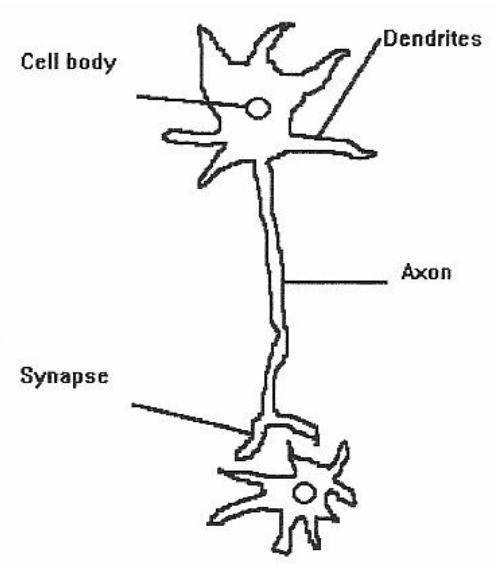

Figure 55 A schematic diagram for a biological neuron [21]

A schematic diagram representing an artificial neuron with bias and no bias is shown in Figure 56. The basic operation of an artificial neuron involves summing its weighted input signals and applying an activation function to generate the output.
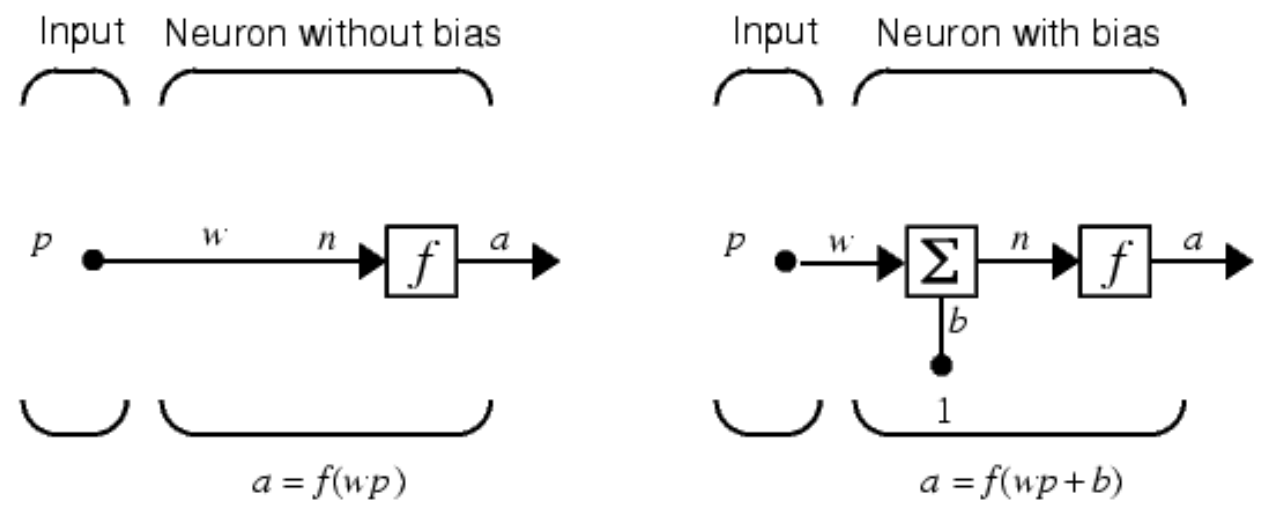

Figure 56 Schematic diagrams for an artificial neuron [21] 
The scalar input $p$ is transmitted through a connection that multiplies its strength by the scalar weight $w$, to form the product $w p$, again a scalar [21]. Here the weighted input $w p$ is the only argument of the transfer function $f$, which produces the scalar output $a$. The neuron on the right has a scalar bias, $b$. the bias can be viewed as simply being added to the product $w p$ as shown by the summing junction or as shifting the function $f$ to the left by an amount $b$. The bias is much like a weight, except that it has a constant input of 1 .

The transfer function net input $n$, again a scalar, is the sum of the weighted input $w p$ and the bias $b$. This sum is the argument of the transfer function $f$. Here $f$ is a transfer function, typically a step function or a sigmoid function, which takes the argument $n$ and produces the output $a$. Examples of various transfer functions are given in the next section. Note that $w$ and $b$ are both adjustable scalar parameters of the neuron. The central idea of neural networks is that such parameters can be adjusted so that the network exhibits some desired or interesting behavior. Thus, the network can be trained to do a particular job by adjusting the weight or bias parameters, or perhaps the network itself will adjust these parameters to achieve some desired end [38][21][19].

\subsection{Backpropagation Neural Networks}

In backpropagation neural networks, input vectors and the corresponding target vectors are used to train a network until it can approximate a function, associate input vectors with specific output vectors, or classify input vectors in an appropriate way as defined. Networks with biases, a sigmoid layer, and a linear output layer are capable of approximating any function with a finite number of discontinuities. Standard backpropagation is a gradient descent algorithm in which the network weights are moved 
along the negative of the gradient of the performance function. The term backpropagation refers to the manner in which the gradient is computed for nonlinear multilayer networks. There are a number of variations on the basic algorithm that are based on other standard optimization techniques, such as conjugate gradient and Newton methods.

Properly trained backpropagation networks tend to give reasonable answers when presented with inputs that they have never seen. Typically, a new input leads to an output similar to the correct output for input vectors used in training that are similar to the new input being presented. This generalization property makes it possible to train a network on a representative set of input/target pairs and get good results without training the network on all possible input/output pairs.

\subsection{Feedforward Networks and Neuron Models}

An elementary neuron with $R$ inputs is shown below Figure 57. Each input is weighted with an appropriate $w$. The sum of the weighted inputs and the bias forms the input to the transfer function $f$. Neurons may use any differentiable transfer function $f$ to generate their output [40], where,

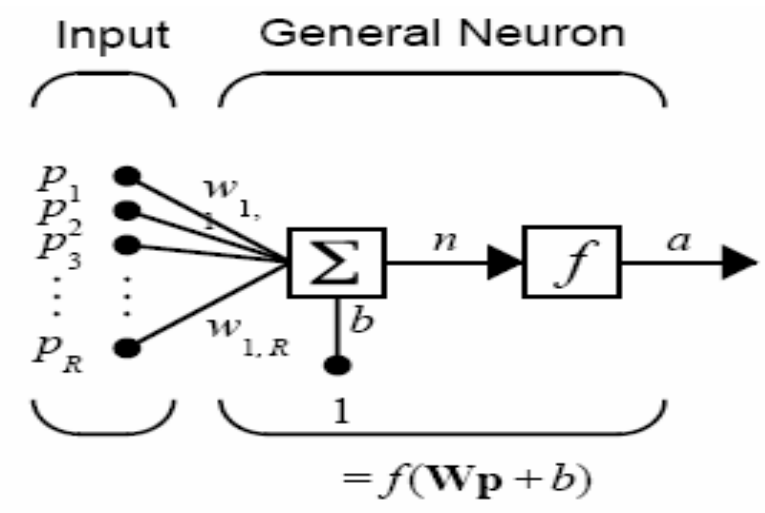

Figure 57 An elementary neuron with $R$ inputs [41]

$R=$ Number of elements in the input vector 
Alternatively, multilayer networks may use the tan-sigmoid transfer function, tansig. If the last layer of a multilayer network has sigmoid neurons, then the outputs of the network are limited to a small range. If linear output neurons are used, the network outputs can take on any value. In backpropagation it is important to be able to calculate the derivatives of any transfer functions used. Each of the transfer functions; tansig, logsig, and purelin have a corresponding derivative function: dtansig, dlogsig, and dpurelin.

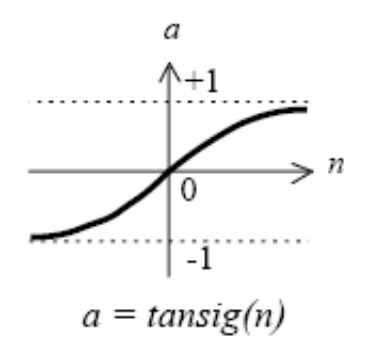

$a=\operatorname{tansig}(n)$
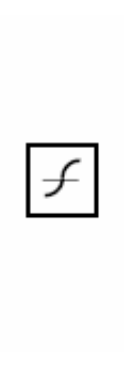

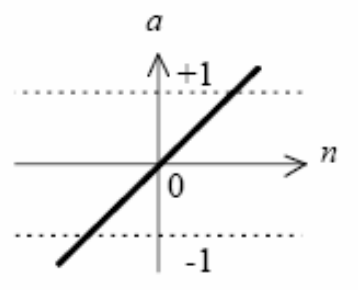

$a=\operatorname{purelin}(n)$

Figure 58 Two transfer functions used in backpropagation neural network analysis [42]

Feedforward networks often have one or more hidden layers of sigmoid neurons followed by an output layer of linear neurons. Multiple layers of neurons with nonlinear transfer functions allow the network to learn nonlinear and linear relationships between input and output vectors, Figure 59. The linear output layer lets the network produce values outside the range -1 to +1 , where $R=$ Number of elements in input vector. 


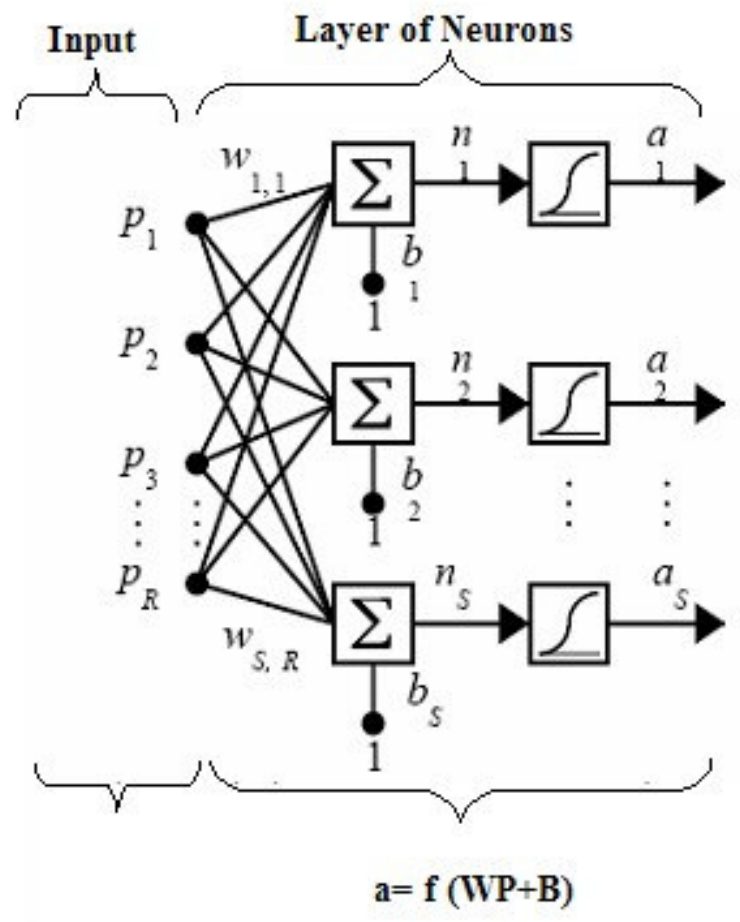

Figure 59 Multiple layers of neurons with nonlinear transfer functions [43][44]

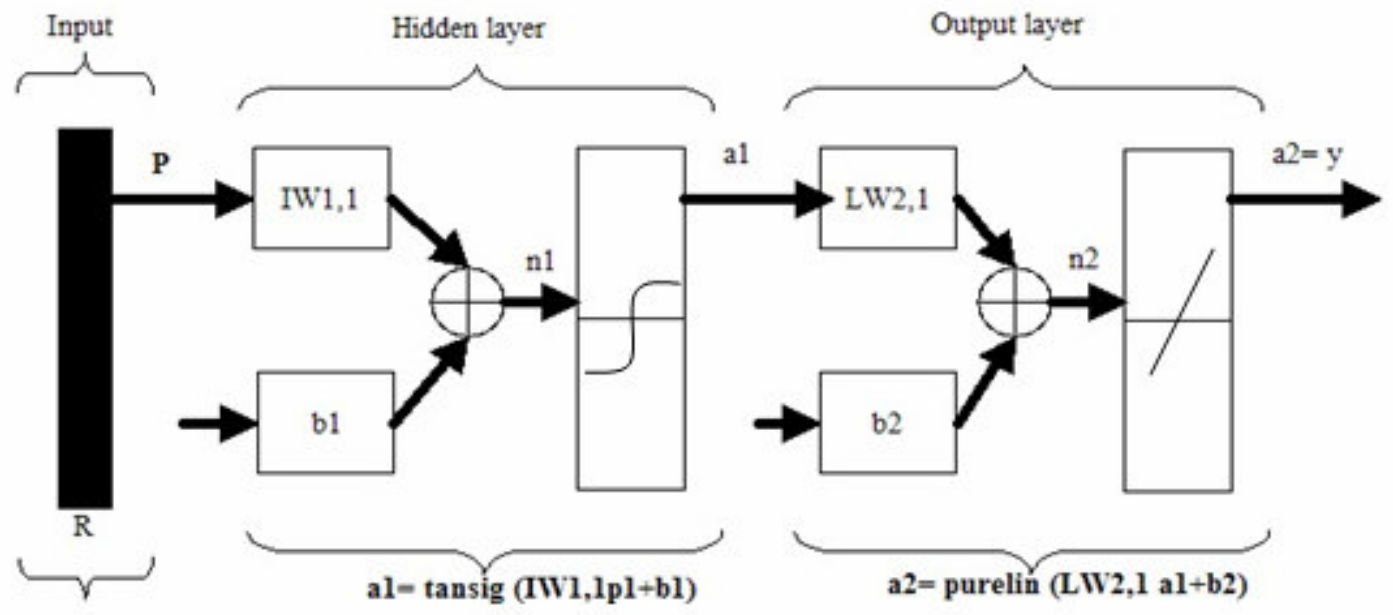

Figure 60 Multiple layers of neurons with one hidden layer and linear output function 
Neural network training can be made more efficient if certain preprocessing steps are performed on the network inputs and targets. This section describes the Principal Component Analysis (prepca, trapca)[38][18].In some analysis, the dimension of the input vector is large, but the components of the vectors are highly correlated (redundant). It is useful in this situation to reduce the dimension of the input vectors. An effective procedure for performing this operation is the principal component analysis. This technique has three effects: it orthogonalizes the components of the input vectors (so that they are uncorrelated with each other); it orders the resulting orthogonal components (principal components) so that those with the largest variations come first; and it eliminates those components that contribute the least to the variation in the data set.

There are generally four steps in the training process [18]:

1- Assemble the training data,

2- Create the network object,

3- Train the network, and

4 -Simulate the network response to new inputs.

Once the network weights and biases have been initialized, the network is ready for training. The network can be trained for function approximation (nonlinear regression), pattern association, or pattern classification. The training process requires a set of examples of proper network behavior - network inputs $\mathrm{p}$ and target outputs $\mathrm{t}$. During training the weights and biases of the network are iteratively adjusted to minimize the network performance function net.performFcn [18]. The default performance function for feedforward networks is mean square error, the average squared error between the network outputs a and the target outputs. 


\subsection{Magnetic Filed Neural Network Analysis}

This section develops a neural network that can predict the crack profile given the magnetic signal. For this purpose the input data needs to be generated for the neural network. Therefore, there are many samples to be generated to train, validate and test the network.

In this analysis the two plate model is used where the magnetic signal produced by the crack is strong and clear. This analysis is carried out for 542 crack profiles as was performed in chapter four. This data will be stored in two libraries one with 121 different crack profiles and the other has 421 different crack profiles. For both libraries, $3 / 5$ of the samples will be used for training the net, $1 / 5$ for validation, and $1 / 5$ for testing the network. A comparison between the two analyses is provided to see the effect of using more sample points on the training process.

In the feedforward backpropagation network, the effect of the hidden layer and the number on neurons on the analysis output is studied. Two networks will be used, one with one hidden layer and a second with two hidden layers. A detailed discussion of the training process and the error calculation is discussed in details.

At the end, the inverse problem is solved, provide with a neural network model that can predict the crack profile in three dimensions.

\subsubsection{Design of the Neural Network}

The flow chart in Figure 61 illustrates the main steps in the network design. First the data is generated for different crack profiles and used to train the network. Once the error margin is acceptable then the net will use $1 / 5$ of the sample points for testing the network. The following subsections will study the different models. 


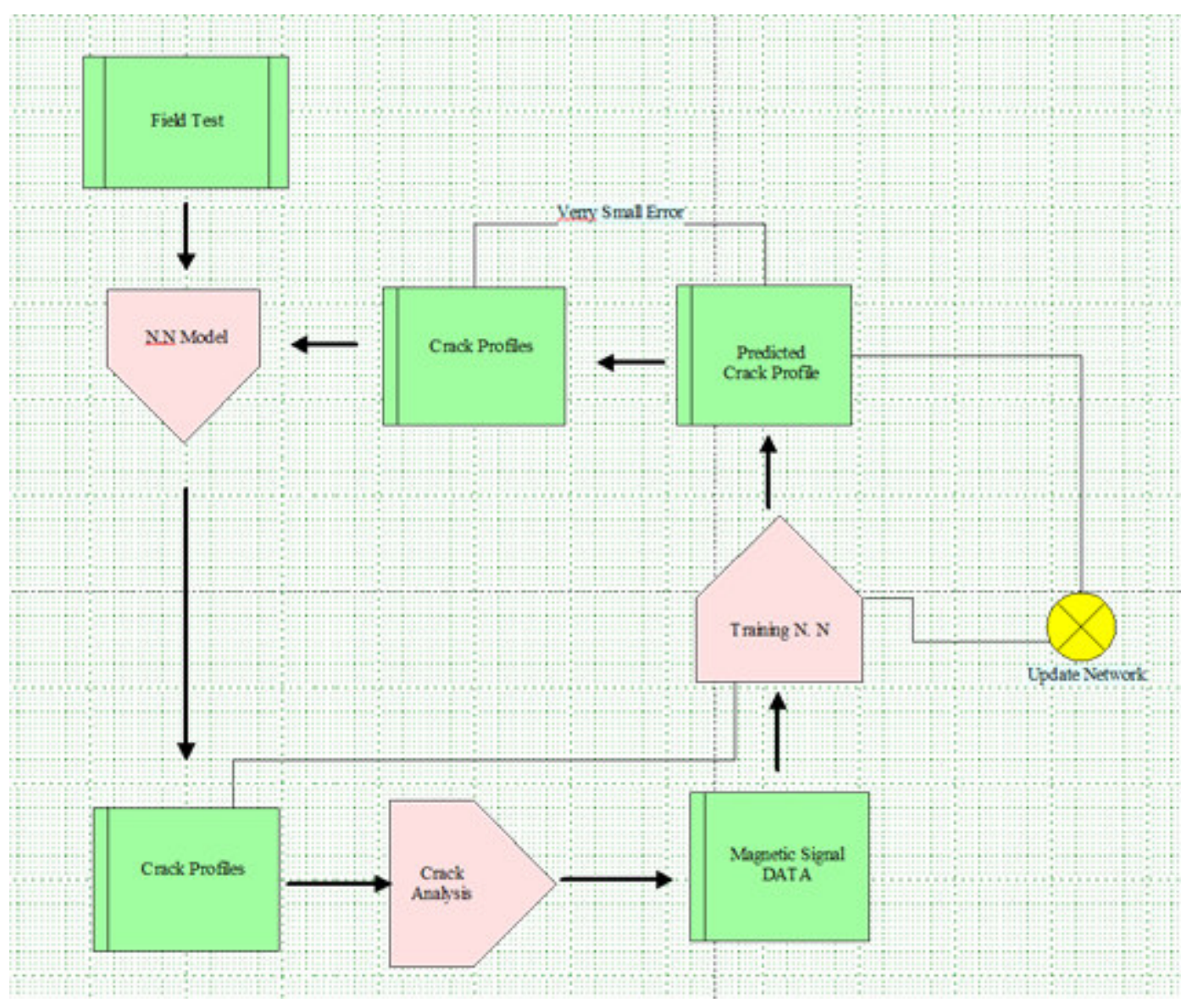

Figure 61 Neural network flow chart design.

\subsubsection{Neural Network with One Hidden Layer.}

\section{Network 1:}

The schematic diagram for a neural network that has only one hidden layer is shown in Figure 62. This network has three layers; input, hidden, and output layers. This network is trained by using 421 samples. The function used in the hidden layer is the sigmoid function and a linear function is used in the output layer. 


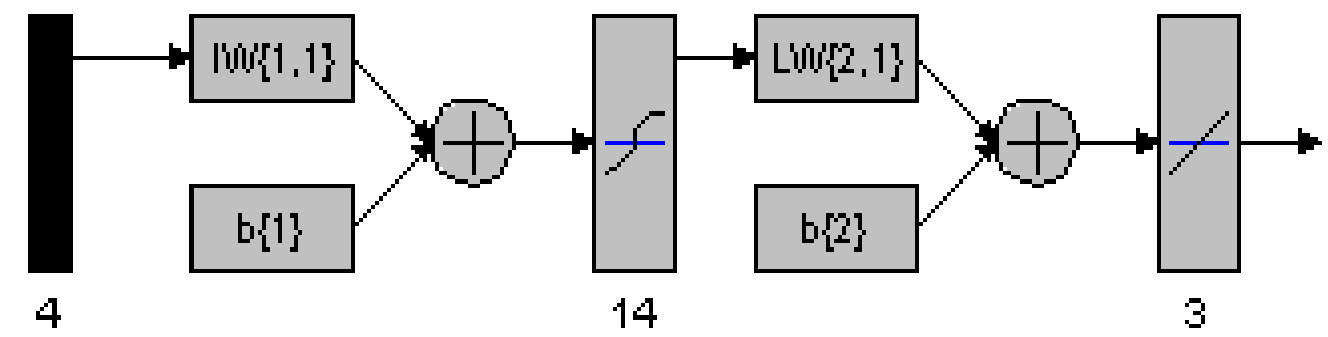

Figure 62 A neural network that has 14 neurons in one hidden layer

The input layer has 4 inputs, down from 81 by using the principle component method as discussed before. It has one hidden layer with 14 neurons. And the output layer represents the crack profile dimensions.

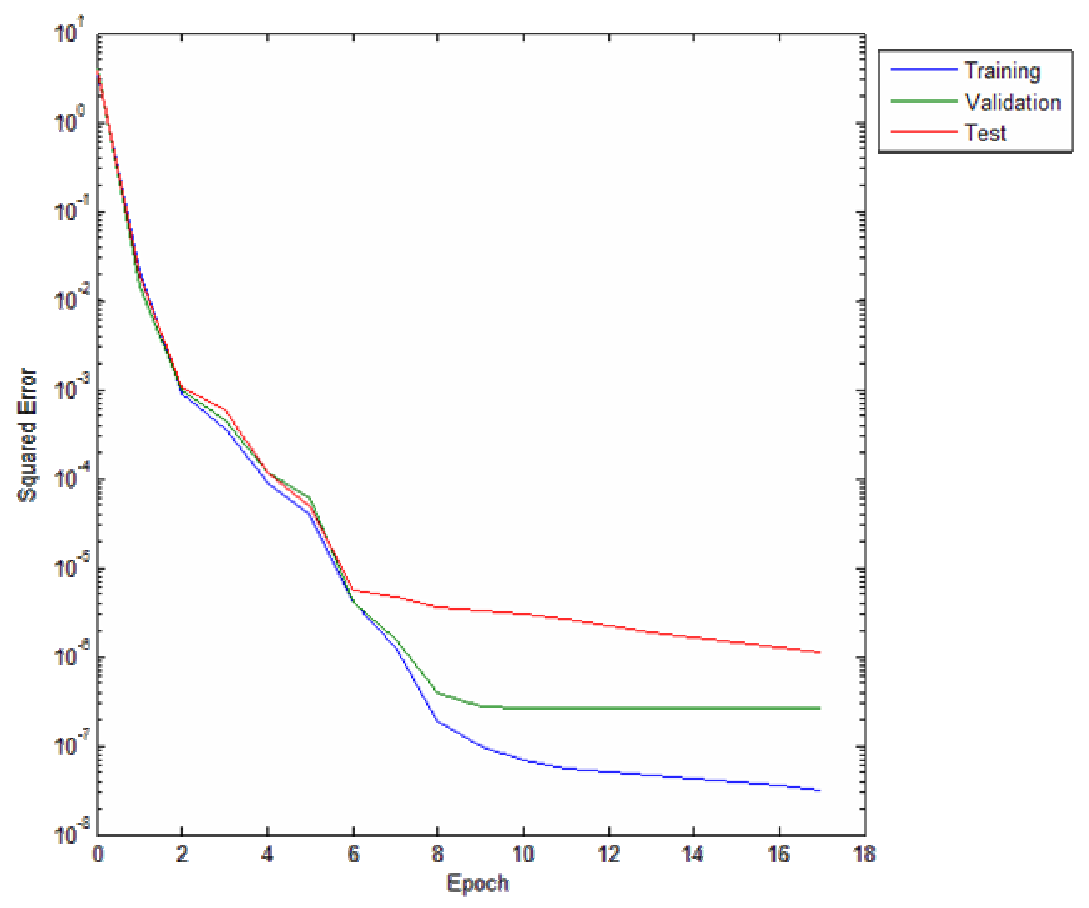

Figure 63 Training, validation and testing process for Net. No. 1

The training process and the error in the crack profile are presented in Figure 63 and Figure 64, respectively. The error in predicting the length is $7.9 \%$, for the width $23.6 \%$ and for the depth is $20.3 \%$. 

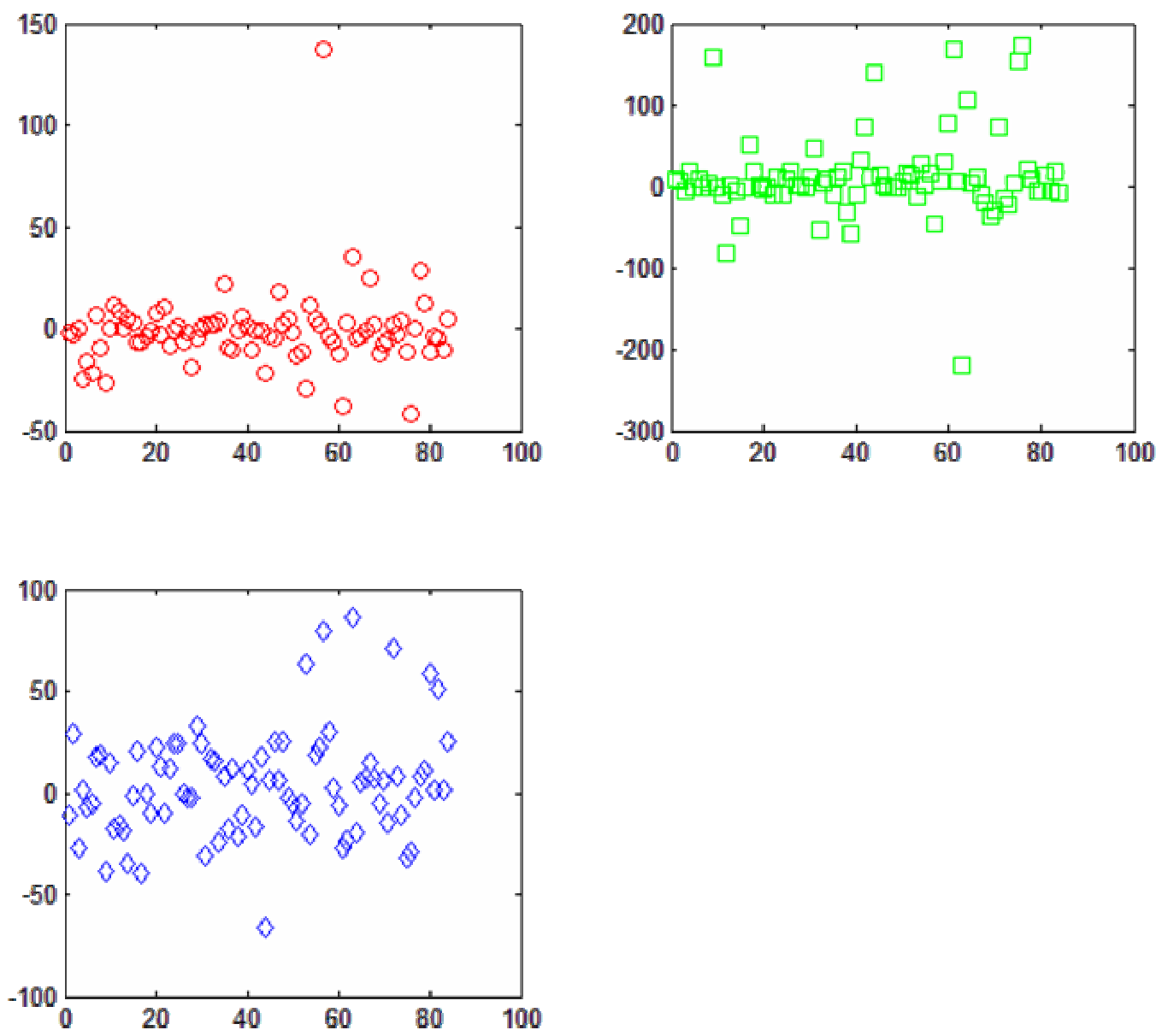

Figure 64 Median Error in predicting crack dimensions, Red for the length. Green for width.and Blue for depth

\section{Network 2:}

The schematic diagram for a neural network that has only one hidden layer is shown in Figure 65. This network has the same functions as Network 1 has. However, Network 2 has more neurons in its hidden layer, 25 neurons. The training process, validation, and testing are presented in Figure 66. The error in predicting the length is $7.7 \%$, the width $17.5 \%$ and the depth is $18.3 \%$. This network has less error but the training process takes more time, about 23 Epochs. 


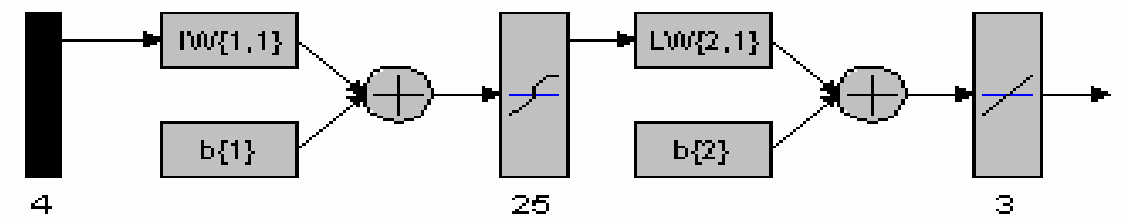

Figure 65 A neural network that has 25 neurons in one hidden layer

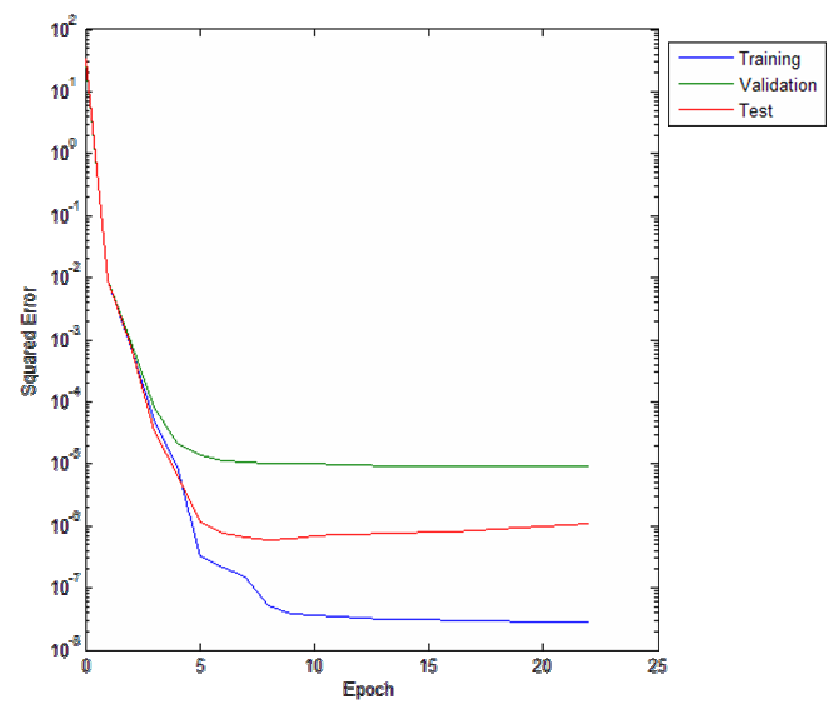

Figure 66 Training, validation and testing process for Net. No. 2

\section{Network 3:}

This network has the same structure design as Network 2. In this case, the network is trained by using two libraries, one with 121 samples and another with 421 samples. 


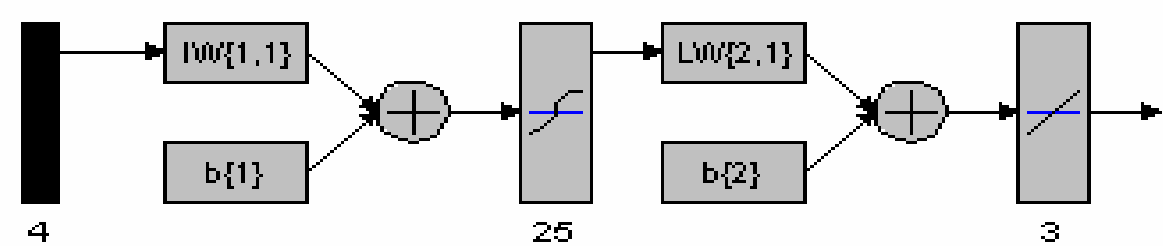

Figure 67 A neural network trained by two sample data libraries.

For training with only 121 magnetic signals, the error in predicting the length is $7.9 \%$, for the width $23.6 \%$ and for the depth is $20.3 \%$. But training the net using the second library that has 421 samples, the error in predicting the length is $5.1 \%$, the width $7.8 \%$ and the depth is $10.3 \%$. The output results for the second library are much better and give close predictions.

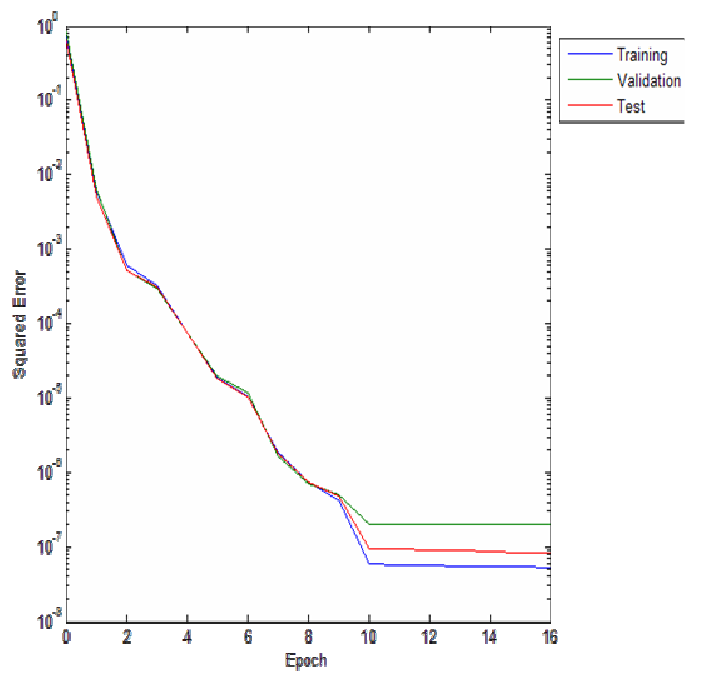

(a)

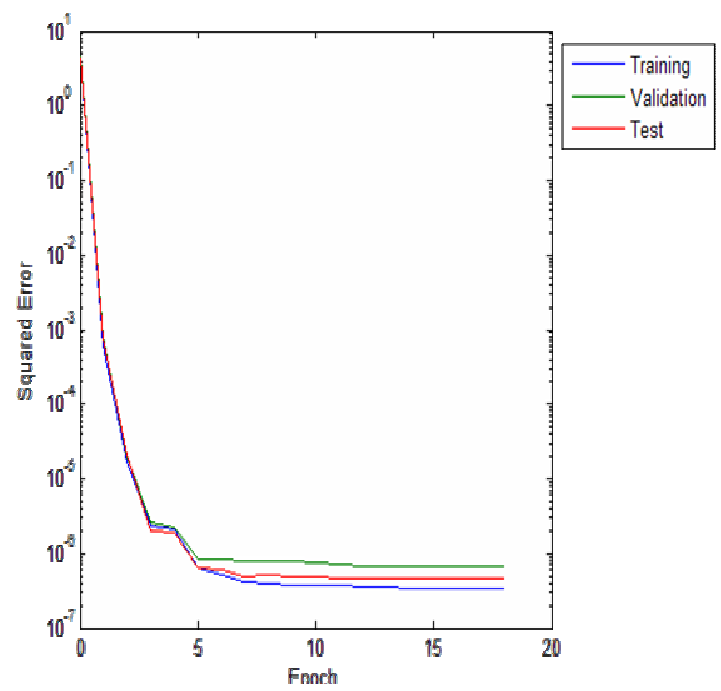

(b)

Figure 68 Training, validation and testing process for Net. No. 3 (a) for 121 library. (b) for 421 library

\subsubsection{Neural Network with Two Hidden Layers}

In this analysis, Neural networks with two hidden layers is designed which gives much better and consistent results. This network has 4 layers in its structure: input layer, two hidden layers, and output layer. The functions are; the sigmoid functions for bother 
hidden layers and a linear function for the output layer. Many neural networks were designed and trained using the 421 samples. In general the results are all acceptable. Two of these networks are listed below.

\section{Network 1:}

This network has two hidden layers with 20 neurons in the first layer and 30 neurons in the second one, Figure 69. Figure 70 shows the training process.

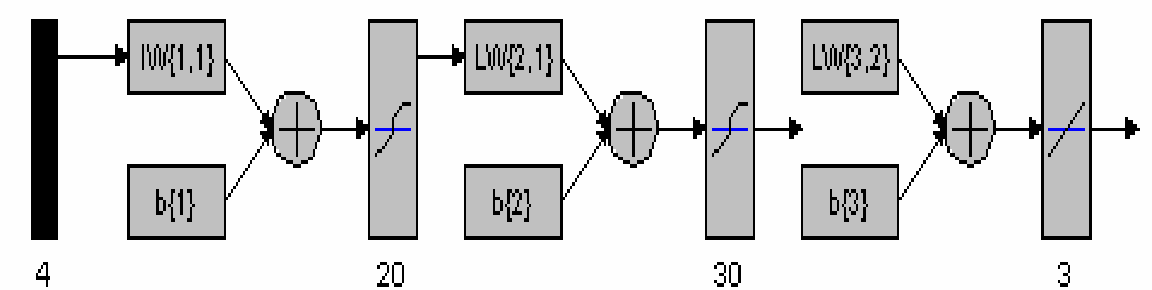

Figure 69 Neural Network with two hidden layers (20 and 30) neurons

The error in the predicting the length is $2.1 \%$, for the width $5.8 \%$ and for the depth is $14.1 \%$. This network has less error but the training process takes more time.

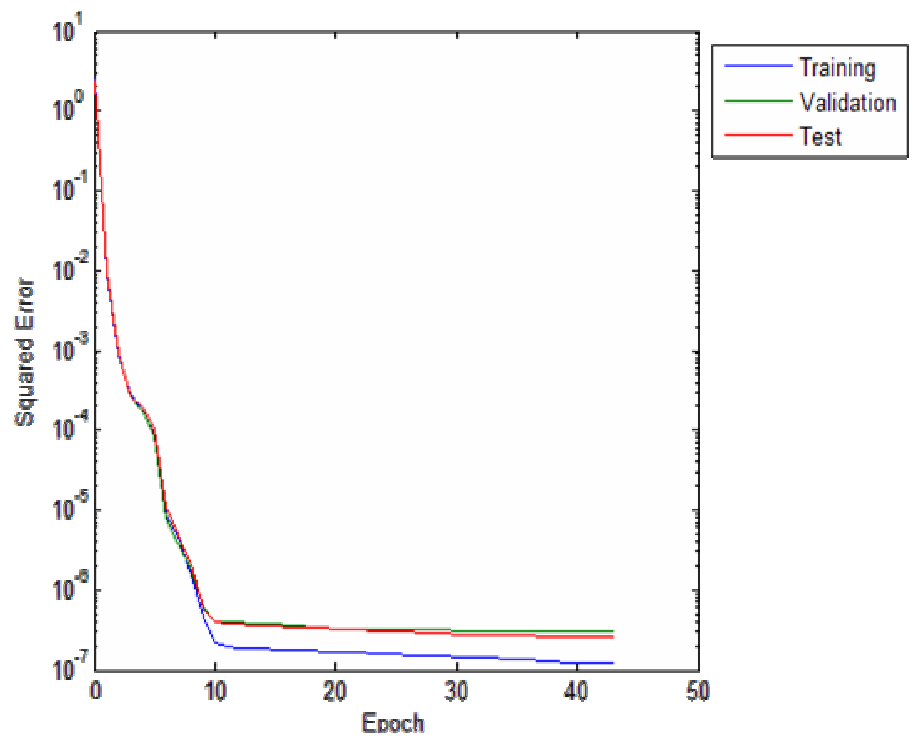

Figure 70 Training, validation and testing process for Net. No. 1 with two hidden layers 


\section{Network 2:}

This network has two hidden layers with 12 neurons in the first layer and 18 neurons in the second one, Figure 71 . Figure 72 shows the training, validation and testing process.

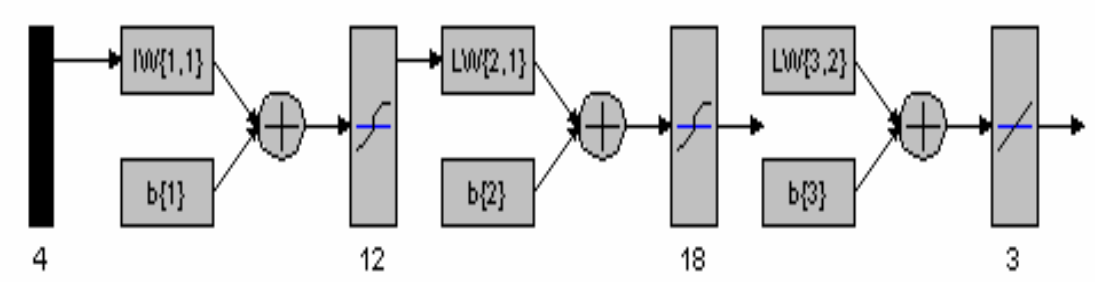

Figure 71 Neural Network with two hidden layers No. 2

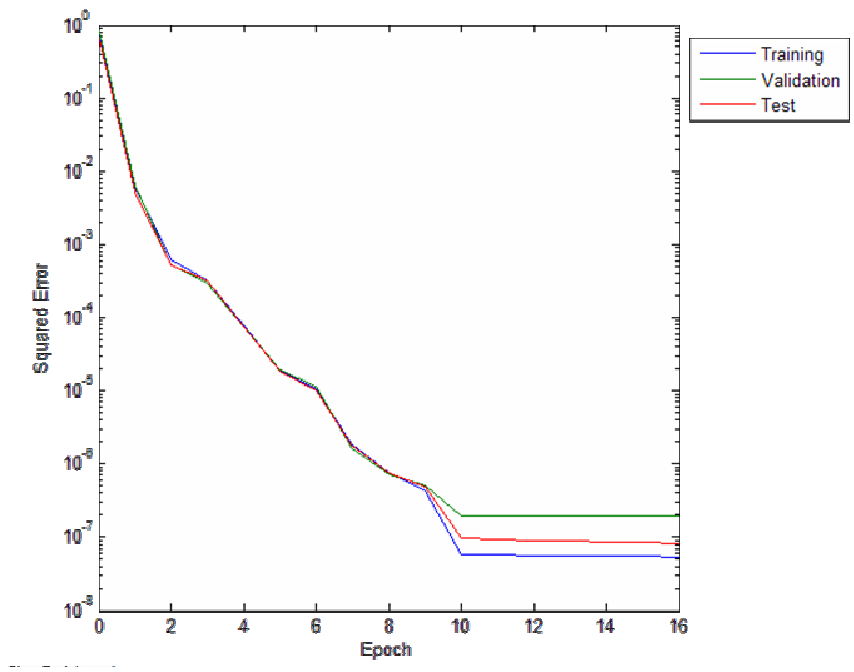

Figure 72 Training, validation and testing process for Net. No.2 with two hidden layers

After running many analyses, the error margin found was less than or around $10 \%$ making it acceptable to be used for solving the inverse problem. One model to be used for future analysis was picked and shown in Figure 71. This model has an error in predicting the length of $1.52 \%$, for the width $5.6 \%$ and for the depth $12.1 \%$. 
This model was tested with a data generated from the simulation analysis using MATLAB that where not used before in any of the previous training process, these data will emulate the data to be collected from the sensor readings around the crack area.

Table 3 shows the actual and predicted profile of three cracks.

\begin{tabular}{|l|l|l|l|l|l|l|l|l|}
\hline \multicolumn{2}{|c|}{ Neural Net. Output (mm) } & \multicolumn{3}{c|}{ Crack Profile (mm) } & \multicolumn{3}{c|}{ Error \% } \\
\hline Length & Width & Depth & Length & Width & Depth & Length & Width & Depth \\
\hline & & & & & & & & \\
0.00297 & 0.00190 & 0.00122 & 0.0030 & 0.0020 & 0.0010 & 0.83 & 5.02 & -22.78 \\
\hline & & & & & & & & \\
\hline 0.00397 & 0.00206 & 0.00128 & 0.0040 & 0.0020 & 0.0010 & 0.57 & -3.45 & -28.68 \\
\hline & & & & & & & & \\
0.00603 & 0.00212 & 0.00118 & 0.0060 & 0.0020 & 0.0010 & -0.53 & -5.97 & -18.82 \\
\hline 0.00270 & 0.00200 & 0.00132 & 0.0027 & 0.0020 & 0.0011 & -0.07 & -0.16 & -20.18 \\
\hline & & & & & & & & \\
0.00413 & 0.00279 & 0.00229 & 0.0041 & 0.0024 & 0.0022 & -0.87 & -16.47 & -4.491 \\
\hline
\end{tabular}

Table 3 Neural Network results for predicting crack profiles

For this neural network analysis, the two plate analysis was used where the signal is not only stronger, also it was very clear from the interference of the edges effect, the magnetic field around the crack is close to zero and the only change there was due to the crack. Conclusions and future work based on this analysis are provided in Chapter Seven. 


\section{Experimental Results and Verification}

In this chapter, experimental analysis is carried out to verify the method concept and the simulation results. Different aluminum plates are used for the experimental analysis; these plates contain different defects with different sizes. The experimental analysis is limited to the tools available for this research. The following subsections discuss the experiment results and give some details about the limitations and error sources that may have affected the results.

\subsection{Experiment Setup}

To carry out the experiment, it is important first to do some preparations and have some understating of the tools limitations. One important thing is the sensor sensitivity to the magnetic field. Detecting small cracks will mainly depend on the sensor reading range and its resolution. The Hall Effect sensor available for this study can read magnetic values from $0.0 \mathrm{G}$ to $30 \mathrm{KG}$ [46], where the minimum value that it can read is $0.1 \mathrm{G}$. From Chapter Four, it was found that the change in the magnetic field due to the cracks is in the range $0.01 \mathrm{G}$ to $0.1 \mathrm{G}$. This means that the sensor will not be able to read this range of magnetic field. Because of the sensor limitation, defects in the aluminum plates with larger sizes were cut to prove the method principles, to verify the modeling results and to test the limits for this sensor.

The experimental setup has three important parts; the sensor, the plate and the electrical circuit. The Experiment setup is shown in Figure 73. 

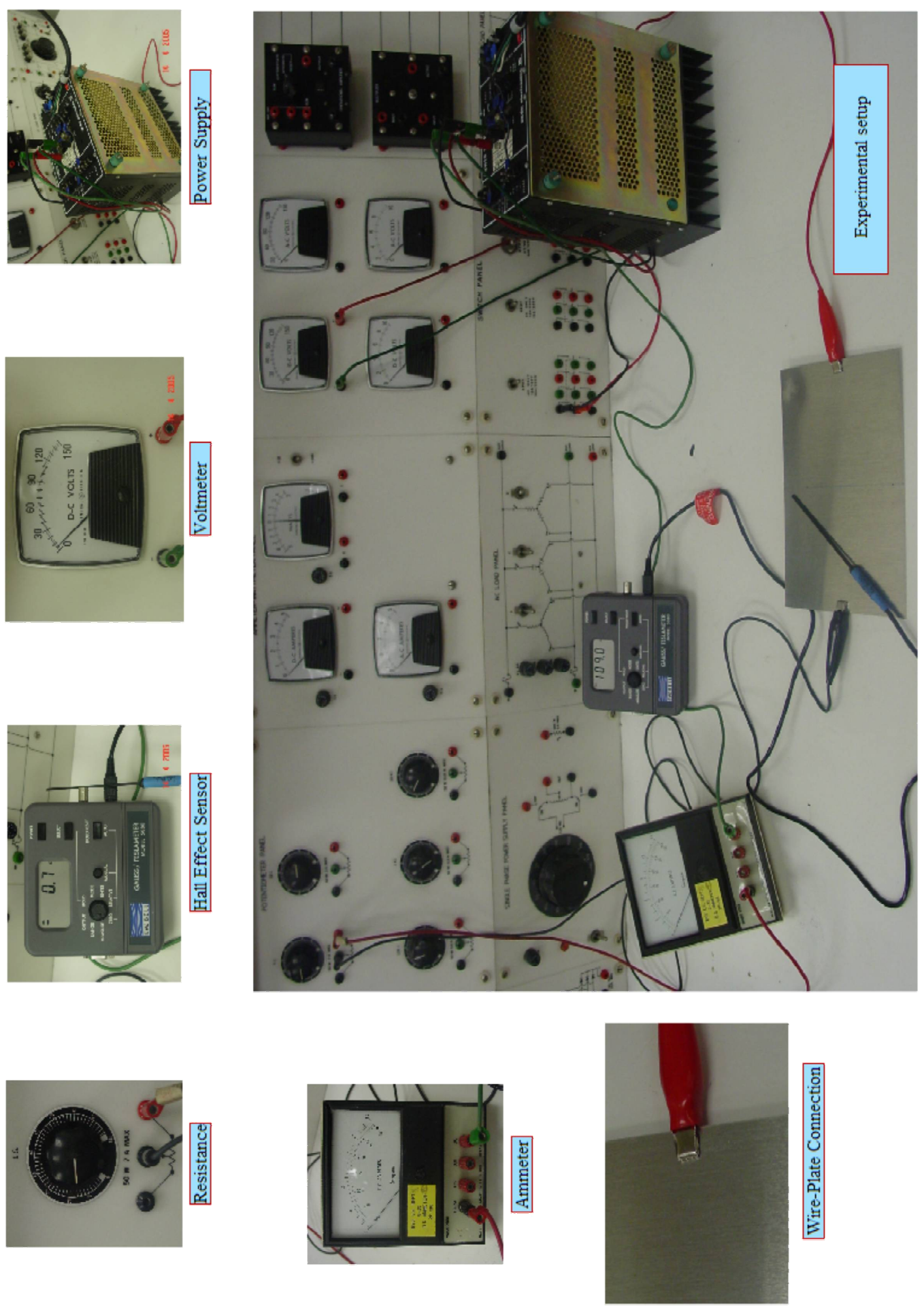

Figure 73 Experimental analysis setup 


\subsubsection{Hall Effect Sensor}

Magnetic field strength is a measure of force produced by an electric current or a permanent magnet. It is the ability to induce a magnetic field " $B$ ". A device commonly used to measure flux density is the Hall generator [45]. A Hall generator is a thin slice of a semiconductor material to which four leads are attached at the midpoint of each edge, Figure 74. A constant current is forced through the material. In a zero magnetic field there is no voltage difference between the other two edges of the sensor probe [46]. When flux lines pass through the material the path of the current bends closer to one edge, creating a voltage difference known as the Hall voltage, $V_{h}$. In an ideal Hall generator there is a linear relationship between the number of flux lines passing through the material (flux density) and the Hall voltage.

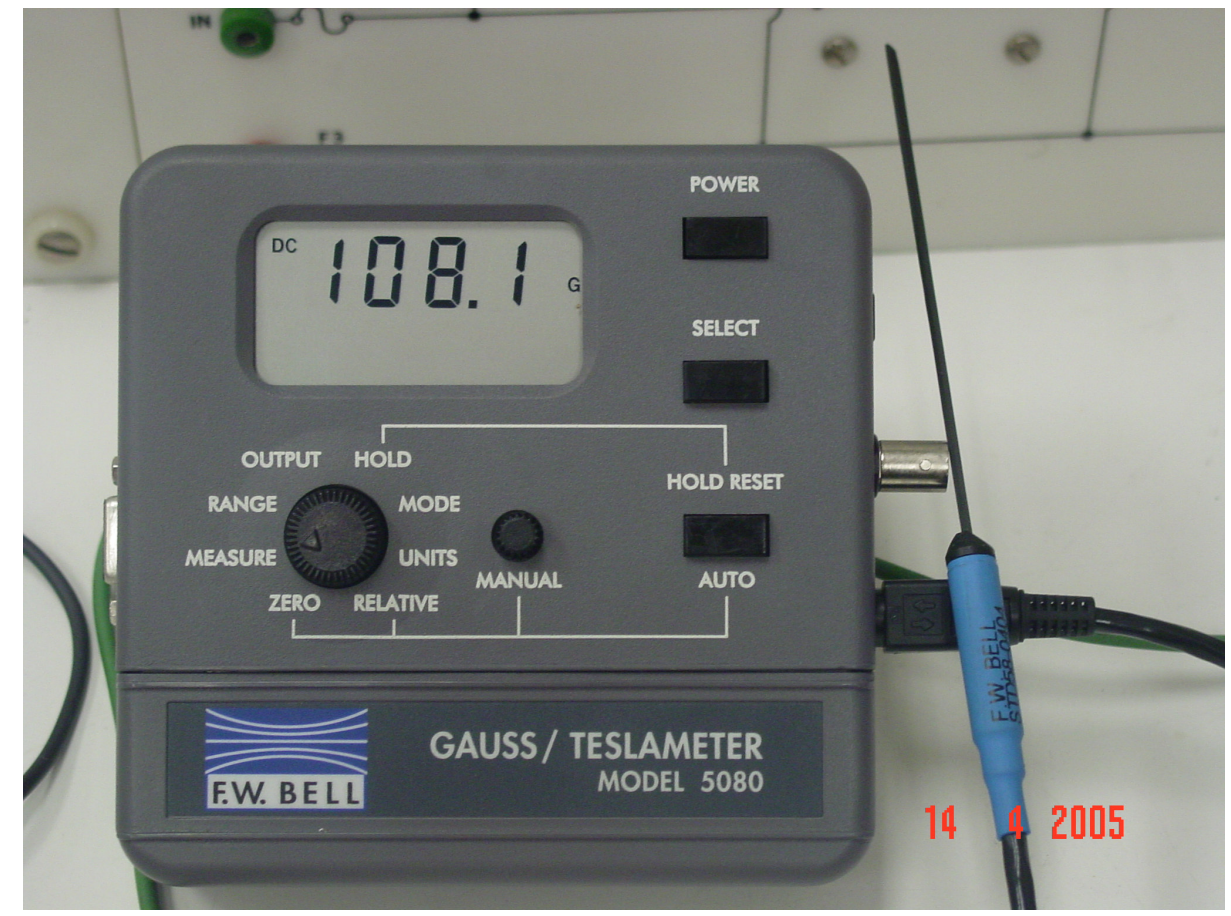

Figure 74 Hall Effect Sensor

The Hall voltage is also a function of direction in which the flux lines pass through the material, producing a positive voltage in one direction and a negative voltage 
in the other. If the same number of flux lines passes through the material in either direction, the net result is zero volts [46].

The Hall voltage is also a function of the angle at which the flux lines pass through the material. The greatest Hall voltage occurs when the flux lines pass perpendicularly through the material. Otherwise the output is related to the cosine of the difference between $90^{\circ}$ and the actual angle.

The sensitive area of the Hall generator is generally defined as the largest circular area within the actual slice of the material. The active area can range in size from $0.2 \mathrm{~mm}$ $(0.008$ ") to $19 \mathrm{~mm}(0.75 ")$ in diameter. Often the Hall generator assembly is too fragile to use by itself so it is often mounted in a protective tube and terminated with a flexible cable and a connector.

One line of flux in the CGS measurement system is called a Maxwell(M), but the weber (W), which is 10 lines. The more commonly used term is the tesla (T), which is 10,000 lines per, $\mathrm{cm}[45]$. Thus

1 Telsa $=10,000$ Gauss

1 Gauss $=0.0001$ Telsa

In the present experiment, a hall sensor model number 5080 gauss/tesla meter is used. This is a portable instrument that utilizes a Hall probe to measure magnetic flux density in terms of gauss, tesla or ampere-meter. The measurement range is from 0.01 $\mathrm{mT}$ (o.1G or o.o1 kAm) to $2.999 \mathrm{~T}$ ( $29.99 \mathrm{kG}$ or $2387 \mathrm{kAm})$ [46].

The MODEL 5080 consists of a palm-sized meter and various detachable Hall probes [46]. The meter operates on standard 9 volt alkaline batteries or can be operated with an external ac-to-dc power supply. A retractable bail allows the meter to stand 
upright on a flat surface. A notch in the bail allows the meter to be wall mounted when bench space is not at a premium. The large display is visible at considerable distances. The instrument is easily configured using a single rotary selector and two pushbuttons. Three measurement ranges can be selected or the meter can automatically select the best range based on the present flux density being measured.

A "zero" function allows the user to remove undesirable readings from nearby magnetic fields (including earth's) or false readings caused by initial electrical offsets in the probe and meter. Included is a "zero flux chamber" which allows the probe to be shielded from external magnetic fields during this operation.

In this study, the magnetic field above the plate before generating the current is 108.9 G. This large field compared to what is expected to be seen from the test current is due to other electrical devices in the Lab. So it is important to "zero" this field before taking any readings with the sensor. The "zero" adjustments can be made manually or automatically.

Other features include three "hold" modes, allowing either the arithmetic maximum, minimum or true peak values to be held indefinitely until reset by the user.

\subsubsection{Electrical Circuit}

This experiment was carried out in one of the Electrical Engineering Dept. Labs at West Virginia University. The electrical circuit is shown in Figure 73. A dc power supply was used to generate a dc current that flows in the plate to produce the electrical field around the crack. The dc power supply available for this ensnarement can generate 5 volts. The resistance of the aluminum plate is very small. Therefore, it was important to use a $1 \Omega$ resistance in series with the plate to protect the power supply. The output 
voltage of the power supply was monitored by using a voltmeter and an ammeter to insure that the electrical circuit was stable. A dc current of 5 Am was generated to pass thru the plate. From chapter four it was found that increasing the current will increase the magnetic field around the crack, and this helps the sensor to detect the magnetic field at the tip of the crack.

\subsubsection{Aluminum Plates}

In order to verify the modeling results, aluminum plates with different cut sizes at the center were fabricated with dimensions of 6 in $\times 9$ in $\times 0.025$ in $(0.1524 \mathrm{~m} \mathrm{x} 0.2286$ $\mathrm{m} \times 0.635 \mathrm{~mm})$. One of these plates is shown in Figure 75. Due to the limitation in the sensor sensitivity and to prove the method concept and verify the modeling results, large cuts were made at the center of the plates to find what minimum cut size that the sensor could detect.

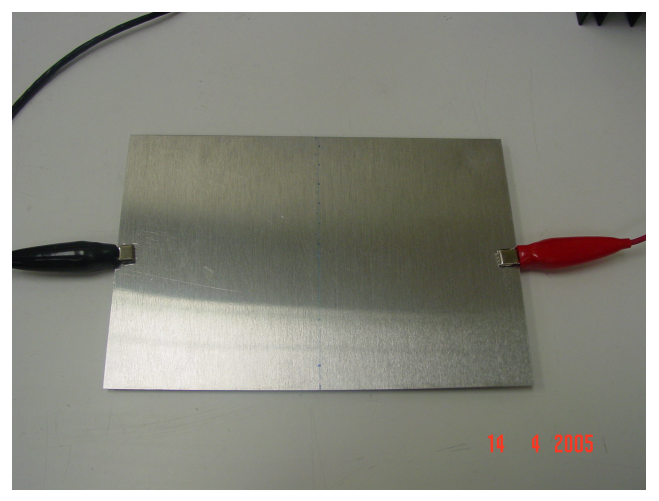

Figure 75 Experimental setup: Plate without cut at the center

Figure 76 shows an aluminum plate with a crack that was $8 \mathrm{~mm}$ in length, $1.5 \mathrm{~mm}$ in width and $0.635 \mathrm{~mm}$ in depth. This plate represents the smallest crack size that the sensor can detect. Using more sensitive sensors with a reading range in $\mathrm{mG}$ or $\mu \mathrm{G}$ will 
allow the sensor to detect cracks with dimensions in micro meters, as shown in Chapter Four.

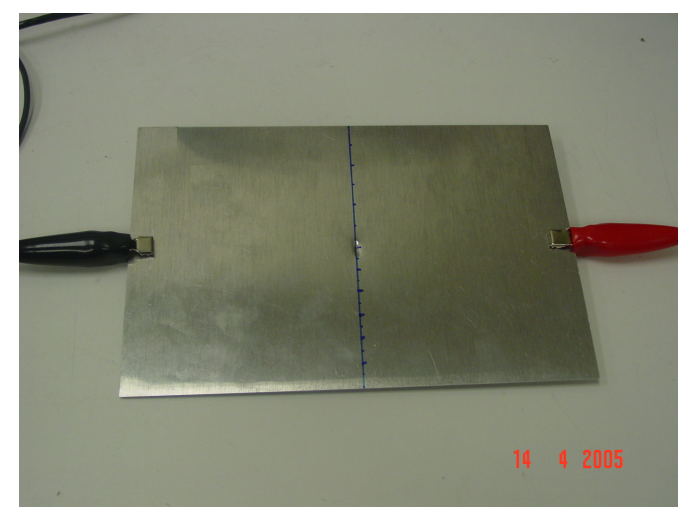

Figure 76 Experimental setup: Plate with a defect at the center

\subsection{Experiment Results}

By conducting the experimental analysis as discussed in the previous sections, the magnetic field above the plate surface and around the defects can be observed. Table 4 shows the magnetic field reading at the defect center, defect edge and close to the plate edges. In Table 4, this first column provides the experiment number. In the next three columns, the plate dimensions were fixed thru all the experimental analysis. In this way the magnetic field produced by the plate edges is not a factor in the detection process, where changing the defect dimensions will be the main factor in changing the sensor reading. Table 4 also provides the defect dimensions at the center of the plate for each experiment case in meters. The test current was fixed at 5 Am value. The sensor magnetic field output in Gauss is provided in the last four columns in Table 4, where the negative values indicate that the direction of the magnetic fields is negative.

The magnetic field from the experimental analysis is compared to the results from the simulation modeling. It is noticed that there is a change in the sensor reading with the 
existence of the defect in the plate, and as the size of this defect increases the corresponding magnetic field at the crack tip is increased, too. The sensor can detect the presence of the defect and locate its center. At plate edges, it is noticed that the magnetic field is almost fixed with a positive direction. There are other magnetic fields that affect the sensor reading from one point to another; more discussion about the reading errors is available in the following sections.

\begin{tabular}{|c|c|c|c|c|c|c|c|c|c|c|}
\hline \multirow[t]{2}{*}{$\begin{array}{l}\text { Exp. } \\
\text { No }\end{array}$} & \multicolumn{3}{|c|}{ Plate Dim.(m) } & \multicolumn{3}{|c|}{ Cut Dim.(m) } & \multicolumn{4}{|c|}{$\begin{array}{c}\text { Magnetic Field Reading Gauss } \\
\text { ( Hall Sensor ) }\end{array}$} \\
\hline & Lp & $\mathrm{Wp}$ & $\mathrm{Tp}$ & $\mathrm{Lc}$ & $\mathrm{Wc}$ & Tc & $\begin{array}{l}\text { Cut } \\
\text { Cent. }\end{array}$ & $\begin{array}{l}\text { Cut } \\
\text { Edg }\end{array}$ & cut/ & $\begin{array}{l}\text { Plat } \\
\text { Edg }\end{array}$ \\
\hline 1 & 0.228 & 0.152 & $0.635 \times 10^{-3}$ & $8 \times 10^{-3}$ & $1.5 \times 10^{-3}$ & $0.635 \times 10^{-3}$ & -0.1 & -0.2 & -0.1 & 0.4 \\
\hline 2 & 0.228 & 0.152 & $0.635 \times 10^{-3}$ & $8 \times 10^{-3}$ & $2 \times 10^{-3}$ & $0.635 \times 10^{-3}$ & -0.1 & -0.2 & -0.1 & 0.4 \\
\hline 3 & 0.228 & 0.152 & $0.635 \times 10^{-3}$ & 0.0254 & 0.0127 & $0.635 \times 10^{-3}$ & -0.1 & -0.25 & -0.1 & 0.4 \\
\hline 4 & 0.228 & 0.152 & $0.635 \times 10^{-3}$ & 0.0762 & 0.0254 & $0.635 \times 10^{-3}$ & -0.1 & -0.3 & -0.2 & 0.4 \\
\hline 5 & 0.228 & 0.152 & $0.635 \times 10^{-3}$ & 0.0762 & 0.0508 & $0.635 \times 10^{-3}$ & -0.1 & -0.35 & -0.2 & 0.4 \\
\hline
\end{tabular}

Table 4 Magnetic field experimental results at certain points around the cuts.

\subsection{Experiment Discussion}

To verify the modeling analysis, an experimental work was conducted as discussed before. Figure 77 shows the sensor magnetic field readings at the cut edges (crack tip) for a sample of the different defect dimensions that are represented in Table 4. The experimental analysis was carried out without using the dummy plate design. 


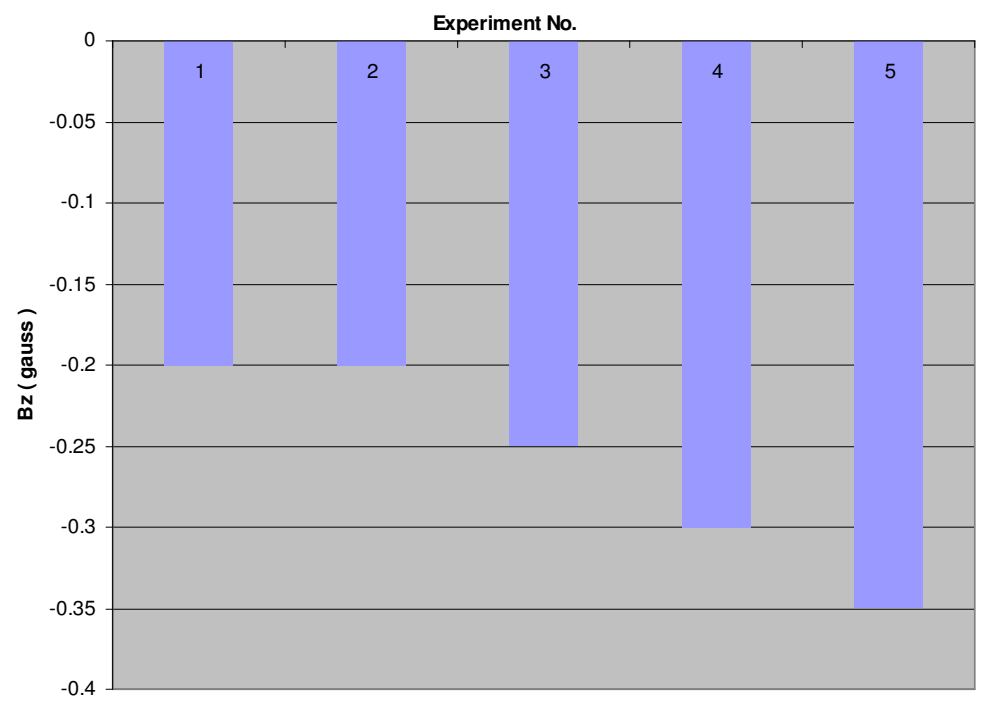

Figure 77 Sensor readings at defect edges

Simulation modeling was carried out for each case that is represented in Table 4 and Figure 77.The simulation results are shown in the following figures. For example, Figure 78 shows the two magnetic fields produced from the test current flowing in the plate. The first field at the middle of the graph is the one produced by the crack where the other field, with the higher value, was produced by the plate edges. It was noticed that the magnetic field produced by the plate edges had almost the same value for all experiment cases.

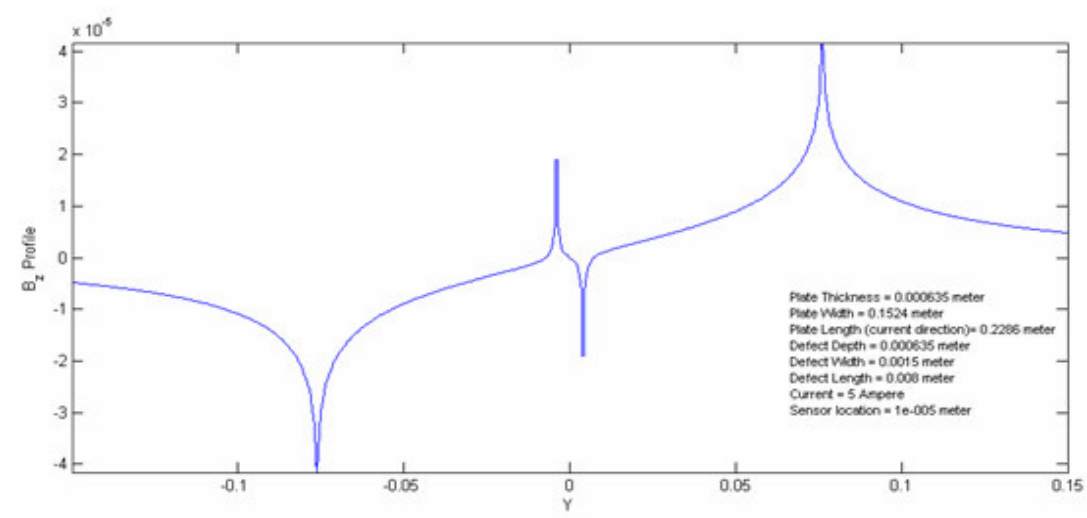

Figure 78 Magnetic fields corresponding to experiment No 2 


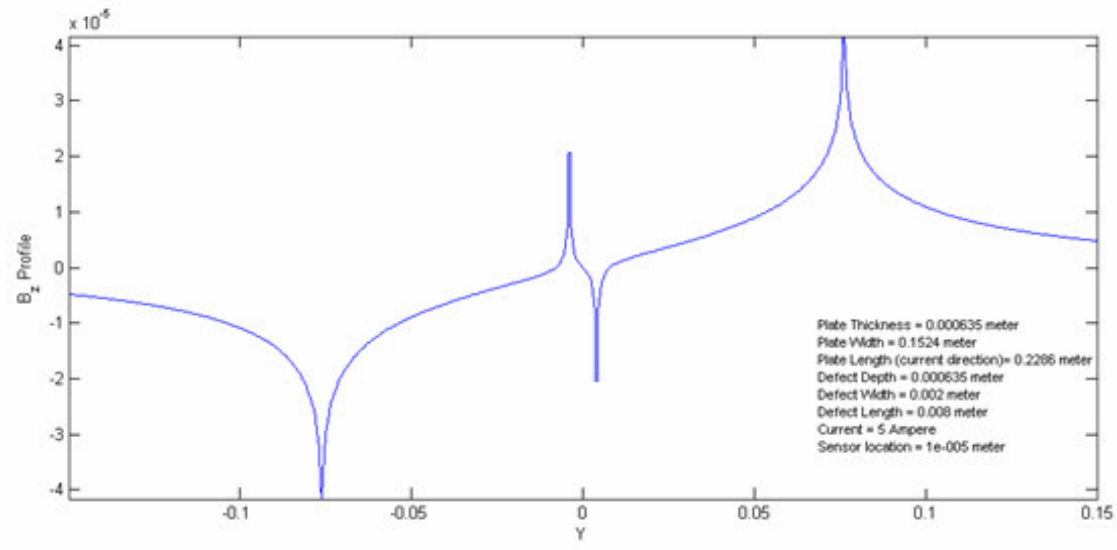

Figure 79 Magnetic fields corresponding to experiment No 3

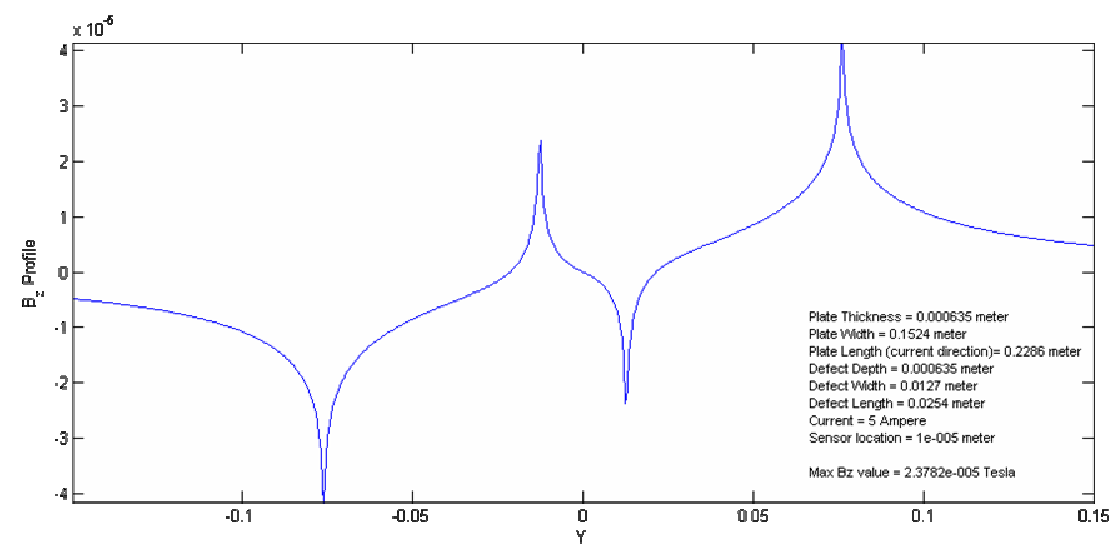

Figure 80 Magnetic fields corresponding to experiment No 4

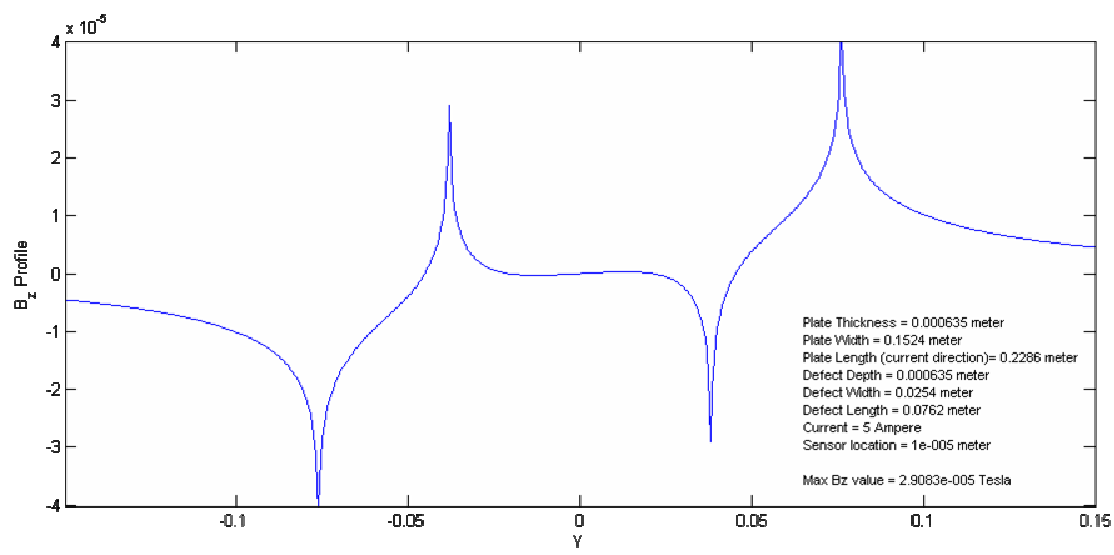

Figure 81 Magnetic fields corresponding to experiment No 5 


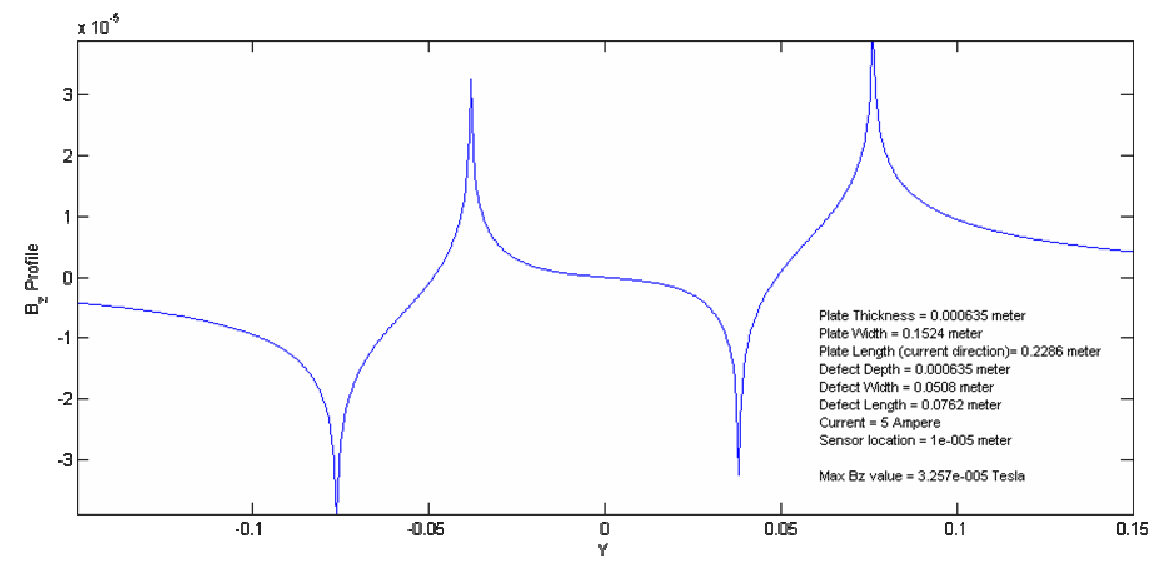

Figure 82 Magnetic fields corresponding to experiment No 6

It is noticed that the sensor is able to detect the change in the magnetic field produced by the defect in the plate and that the magnitude of this magnetic field is changing with the change in the defect dimensions. Figure 83 shows a comparison between the experimental and modeling analysis results.

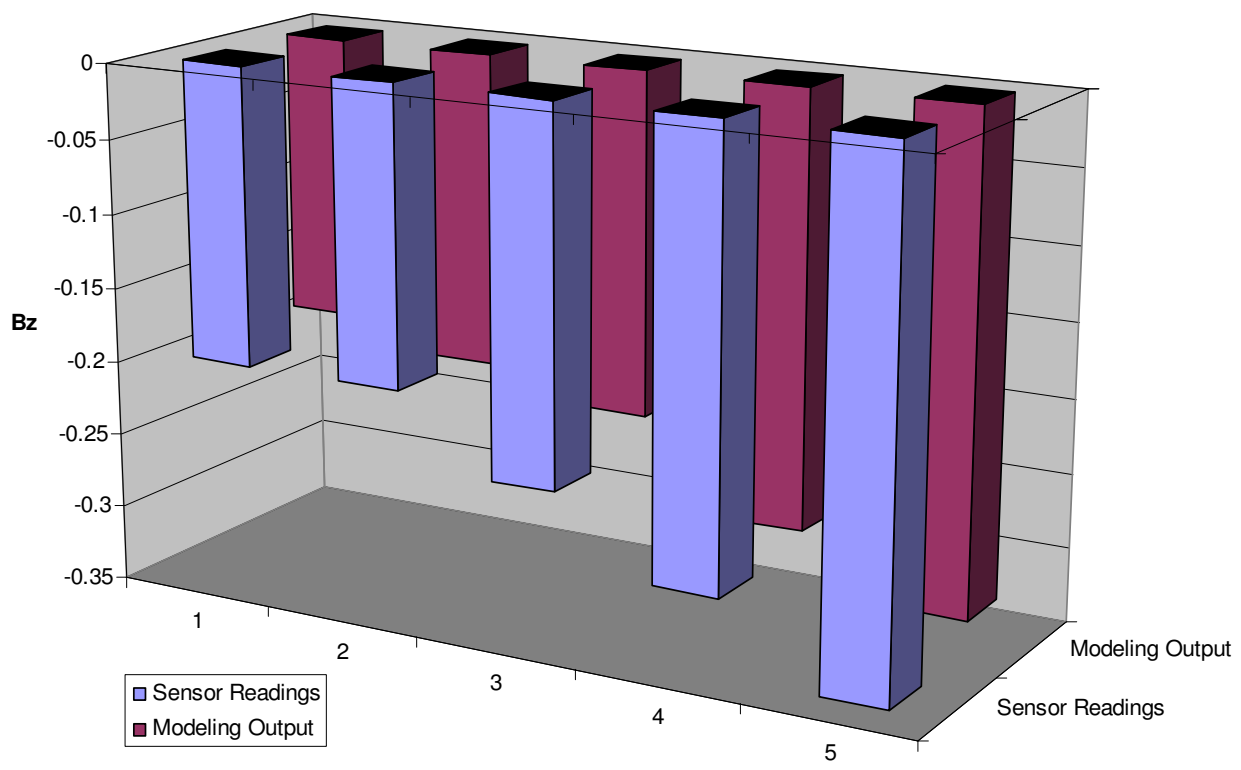

Figure 83 A comparison between the experiment and modeling results

Figure 84 shows the percentage errors between the sensor and modeling results. The percentage error is in a range from $3.3 \%$ to $8.6 \%$. 


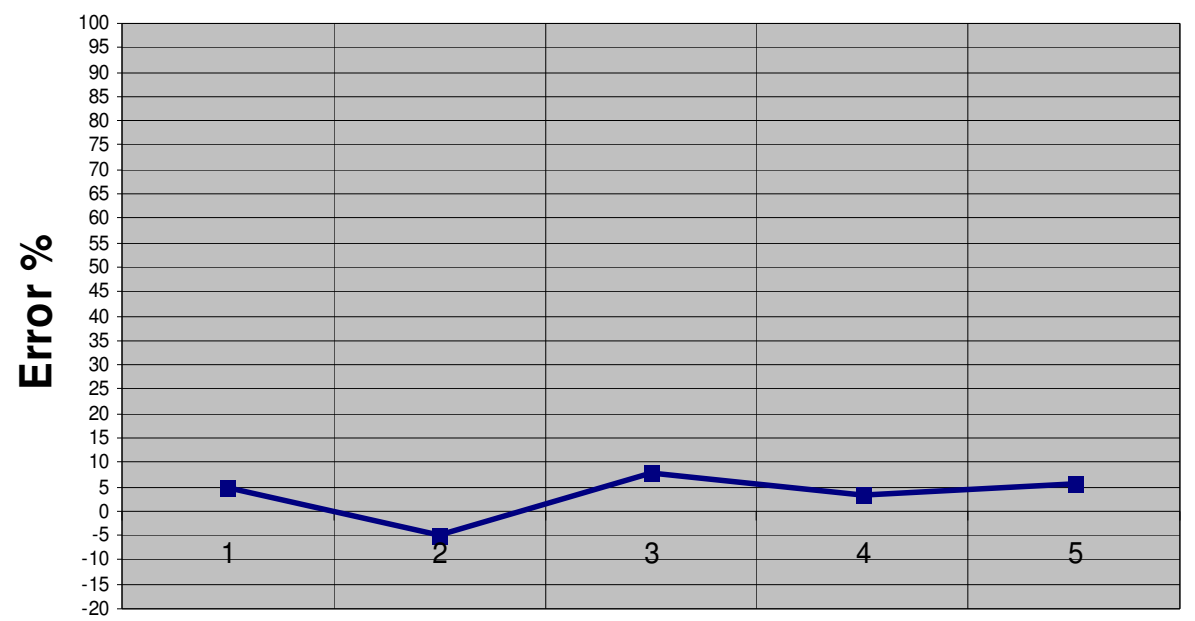

\section{Experiment No.}

Figure 84 Percentage errors between the sensor and modeling results.

This difference in values is due to certain error sources. First, due to the sensor resolution, it was not visible to detect the small changes in magnetic field in the case of small cracks. The magnetic field in the space above the plate center before applying the current to the plate is about 108.9 , and this value is slightly changing within a range of $1 \mathrm{G}$ when the sensor location above the plate changes. The use of the "zero" function in the sensor to cancel this reading is an important step in the experimental analysis. The zeroing function needs to be repeated before each sensor reading of magnetic field after applying the current.

The plate edges produce a magnetic field that interferes with the sensor readings of the magnetic field produced by the defect. Another source of error is the magnetic field produced by the wires carrying the current to the plat and the way these wires were connected to the plate. It was found that the value of the magnetic field produced by the current in the connection wires to be about $2.6 \mathrm{G}-6.7 \mathrm{G}$ depending on the distance from 
the wire interface with the plate. Any movement in the circuit wires will result in a huge change in the magnetic field.

The location of the sensor above the plate is also an important factor in the sensor readings. It is important to keep this distance fixed; otherwise any small change will result in a big change in the magnetic field.

One way to minimize the errors is to conduct the experiment a distance from the interference of other magnetic fields produced by other electrical parts of the circuit. Using a sensitive sensor that has a resolution in $\mathrm{mG}$ or $\mu \mathrm{G}$ will provide the capability to read very small changes in the magnetic field.

In summary, the experimental analysis shows clearly that the modeling results are valid and the sensor can detect the changes in the magnetic field with the changes in the defect dimensions. The sensor was able to detect the defect center and locate the defect edges. 


\section{Conclusions and Recommendations}

This chapter presents the final conclusions of this work and gives recommendations for future work in this field of research.

\subsection{Conclusions}

The goal of this study was to develop a system that can solve the nondestructive evaluation problem to detect cracks or defects in aircraft structures by using a magnetic flux leakage method that was developed for non-ferromagnetic structures. Neural network models were used to predict the crack profile in the three dimensional space by solving the inverse problem. Available neural networks address the problem in two dimensions only. In another word, it predicts two dimensions of the crack profile. This study proposed a tool that can predict the crack profile in its three dimensions given that the dimensions are uniform. This will give better understanding of the crack behavior in the structure.

The nondestructive method is proposed by measuring that magnetic flux on the top of the conducting plate. The magnetic field distribution on the top of the plate is calculated by considering the current density distribution in the plate. The current density has high values around the crack and these produce high magnetic fields. At the crack tip, the magnetic field has its maximum value, this helps to predict the crack tip location. Under mechanical loads the crack will grow and change geometry. Changing the length of the crack will result in a change in the magnetic field at the tip of the crack. By monitoring the magnitude of the magnetic field at the crack, the crack length or the crack propagation can be monitored and based on this information the expertise can decide if the structure is in a critical stage or not. 
Any change in the crack geometry will cause a change in the magnetic field around the crack. This change is modeled in the three dimensional space to give better understanding of the magnetic signal. The effect of changing any of the crack dimensions on the magnetic signal at the crack tip is discussed, where the change in any dimension of the crack will change the magnitude of the field at the crack tip and the shape of the signal profile.

Other magnetic fields can affect the sensor readings of the magnetic field above the crack or at the crack tip. The magnetic fields from the plate edges and the wires that carry the current have higher values than the values of the magnetic field produced by the crack. This serious problem was discussed and some recommendations were given to minimize this unwanted effect. A new plate, called a dummy plate, is used to cancel or minimize the effect of the magnetic fields produced by the plate edges. This plate is identical to the plate under testing but free from any crack in its structure. If the current pass in the dummy plate is in an opposite direction to the current in the plate under testing, a new magnetic field will result from the dummy plate edges in an opposite direction to that produced by the master plate edges.

The effectiveness of the dummy plate was proven. The change in the magnetic signal around the crack was clear, where the value of the magnetic field is close to zero. As a result, the crack center can be detected, and the crack tip can be identified.

The other problem was the magnetic fields produced by the wires carrying the current. To deal with this, it is recommended to connect the wires in a perpendicular way to the plate surface, so their magnetic fields are not normal to the plate surface and they do not interfere with the sensor reading. 
Using simulation models to generate magnetic fields around different crack profiles, gives the ability to understand the nature of these fields and provides enough information to use for the neural network analysis to solve the inverse problem where the crack profile can be detected.

The neural network model was designed such that it can be used to detect cracks and decide their dimensions, length, width and depth in the plate. The Neural network model gives good results in predicting crack profiles. The sensor, the dummy plate and the neural network model can be integrated in one system that can scan structures for crack or defects where there is no need for a computer at the time of testing to analyze the output of the sensor. The sensor can be mounted on the dummy plate to scan the structure under testing.

An experimental work was conducted to verify the modeling results before using the dummy plate design. Detecting the magnetic field above the crack depends on the sensor reading range and the crack size. Magnetic field calibrations need to be done before each reading to minimize the errors in sensor readings. The sensor was able to defect the defects and locate its center and tips.

It is important to mention that even this new method has the capability to detect different cracks in the structure; the analytical solution, the magnetic field analysis, and the neural network design were limited to certain plate and crack geometries. The crack has uniform directions and always normal to the current direction.

As a result of this work, a tool has been designed that can solve the nondestructive evaluation problem and the inverse problem in three dimensions to give the capability to 
provide an enhanced assessment tool for judgment and decision-making which will improve the safety of metallic structures and save people lives.

\subsection{Recommendations}

Evaluation of crack profiles based on experimental signals can be performed for plates with previously machined cracks. However, natural cracks such as fatigue cracks must be treated. Natural cracks are not only very complicated in shape, but also appear in clusters having a complex conductivity distribution. The problem of separating signals coming from several different cracks and modeling cracks with complicated shapes and unknown conductivity distributions present a challenge for future work.

The neural network model can predict crack profiles from magnetic signals produced by the suggested crack geometry, uniform length, width and depth. Other models need to be developed for other possible crack profiles, like the $\mathrm{V}$ shape or the semi elliptical shape. If the crack is not uniform in any of its dimensions this will raise a challenge in designing the neural network model. 


\section{References}

[1] M. Goktepe. Nondestructive Crack Detection by Capturing Local Flux Leakage Field, Sensors and Actuators, vol. 91, 2001, pp70-72.

[2] J. blitz. Electrical and Magnetic Methods of Nondestructive Testing, Adam Hilger, 1991.

[3] T. Nonaka, et al. Nondestructive Detection of Cracks in a Distribution Line by Evaluation Magnetic Field Distribution, IEEE Transaction on Magnetics, vol.39, no. 5, September 2003.

[4] E. Gelenbe. Neural Networks: Advances and Applications II, Amsterdam, North Holland, 1992.

[5] B. Kosko. Neural Networks for Signal Processing, Prentice Hall, Englewood Cliffs, NJ, 1992.

[6] P. Ramuhalli, et al. Neural Network Based Inversion Algorithms in Magnetic Flux Leakage NDE. Journal of Applied Physics, vol. 93, no. 10, 15 May 2003, pp 8274-8276.

[7] T. Warner, et al. Improved Durability Aluminum Alloys for Airframe Structures, RTO MP-25, April 1999.

[8] J. Qu. Crack Detection in Thick Annular Components Using Ultrasonic Guided Waves. Proc. Instn. Mech. Engrs. Vol. 214, part C, pp 1163-1171

[9] S. Calcagno, A Novel Approach for Detecting and Classifying Defects in Metallic Plates. IEEE Transaction on Magnetics, vol.39, no. 3, May 2003, pp 1531-1534

[10] B. Wincheski. Electromagnetic Detection of Fatigue Cracks Under Protruding Head Ferromagnetic Fasteners. http://techreports.larc.nasa.gov/ltrs/PDF/1999/mtg/NASA-99-3caa-raw.pdf

[11] A. Mal, et al. NDE of Hidden Flaws in Aging Aircraft Structures Using Obliquely Backscattered Ultrasonic Signals (OBUS). NDT.net, January 1999, vol. 4, no. 1

[12] W. Winfree. New Nondestructive Techniques for Inspection of Aircraft Structure. SPIE vol. 2945, pp 88-95.

[13] A. khan. Nondestructive Testing Applications in Commercial Aircraft , NDT.net vol. 4, no. 6, June 1999, pp 1-9 
[14] Shan. Surface-Breaking Fatigue Crack Detection Using Laser Ultrasound. Applied Physics Lett. 62 (21) 24 May 1993 pp 2649-2651.

[15] M. Lasser, et al. Latest Development in Real-time 2D Ultrasound Inspection for Aging Aircraft. NDT.net vol. 4, no. 1, January 1999.

[16] I. Komura, et al. Crack Detection and Sizing Technique by Ultrasonic and Electromagnetic Methods. Nuclear Engineering and Design, vol. 206, 2001, pp 351-362.

[17] M. Mizell and W. Linndy Jr., In-flight Crack Detection System for the C-135 Lower Center Wing Skin. ISA ASI 76240, 1976, pp 259-268

[18] The MathWorks, Inc., MATLAB Software Documentation: Neural Network Tool Box.

[19] M. Anthony and P. Barteltt. Neural Network Learning: Theoretical Foundations. Cambridge University Press, Cambridge, 1999.

[20] S. Haykin. Neural Networks: A Comprehensive Foundation, New York, Macmillan, 1994.

[21] A. Kulkarin. Computer Vision and Fuzzy Neural Systems, Eastman Kodak Company, Rochester, NY, 2001

[22] W. Kern. Magnetic Crack Detection Using Combined AC Field Magnetization. C.S.N.D.T Journal, July-Aug 1986, pp 46-49.

[23] A. Portis. Electromagnetic Fields: Sources and Media, Wiley, New York, NY, 1976.

[24] P. Clemmow. An Introduction to Electromagnetic Theory. Cambridge University Press, London, 1973.

[25] P. Hammond, Electromagnetism for Engineers, Oxford University Press, 1997.

[26] A. Hussain. Magnetic Field of a Direct Current in a Cylindrical-Shaped Conductor Imbedded in a Resistive Half-Space Beneath a Conductive Surface Layer, Journal of Applied Physics, 11/15/89, Vol. 66 Issue 10, p4587

[27] E. Della. And C. Longo. The Electromagnetic Field, Allyn and Bacon, Boston, 1969.

[28] W. Smythe. Static and Dynamic Electricity, McGraw Hill, New York, 1939.

[29] J. Jackson. Classical Electrodynamics, John Wiley \& sons, New York, 1975. 
[30] H. Lui, et al. Analysis of Interaction of Multiple Cracks in Direct Current Field and Nondestructive Evaluation. Transaction of the ASME, vol. 66, June 1999, pp 468-475.

[31] W. Dover. Use of AC field Measurements in Crack Detection. Measurement and Inspection Technology, March 1982, pp 32-35.

[32] T. Chady, et al. Crack Detection and Recognition Using an Eddy Current Differential Probe. IEEE Transaction on Magnetics, vol. 35, no. 3, May 1999, pp 1849-1852.

[33] J. Bowler. Review of Eddy Current Inversion with Application to Nondestructive Evaluation. International Journal of Applied Electromagnetics and Mechanics, 8, 1997, pp 3-16.

[34] H. Neff. Basic Electromagnetic Fields, New York, Harper \& Row, 1981.

[35] G. Filippopoulos and D. Tsanakas. Analytical Calculation of the Magnetic Field Produced by Electrical Power Lines. IEEE Transactions on Power Delivery, April 2005, Vol. 20, Issue 2, p1474.

[36] T. Nonaka, et al. Magnetic Nondestructive Detection of Crack in Distribution Line. IEEE Transaction on Magnetics, vol. 37, no. 4, July 2001, pp 2746-2748.

[37] J. Wikswo. The Calculation of Magnetic Field from a Current Distribution: Application to Finite Element Techniques, IEEE Transactions on Magnetics, MAg-14: 1076-1077, 1978.

[38] K. Miya, Recent Advancement of Electromagnetic NDE in Japan, IEEE Transaction on Magnetics, vol. 8, no. 2, March 2002, pp 321-326

[39] P. Lisboa. Neural Networks: Current Applications, Chapman and Hall, London, 1992.

[40] W. Bond, et al. Neural Network Analysis of Nondestructive Evaluation Patterns. Proceedings of the 1992 ACM/SIGAPP Symposium on Applied Computing, Kansas City, Missouri, 1992, pp 643-650.

[41] R. Lippmann. An Introduction to Computing with Neural Nets, IEEE ASSP Magazine, April 1987, pp 4-22

[42] V. Honavar and L. Uhr. Artificial Intelligence and Neural Networks: Steps Toward Principled Integration, Academic Press, Boston, 1994. 
[43] D. Hudson and E. Maurice. Neural Networks and Artificial Intelligence for Biomedical Engineering, Institute of Electrical and Electronics Engineers, New York, 2000.

[44] J. Freeman and M. David. Neural Networks: Algorithms, Applications, and Programming Techniques, Addison-Wesley, Reading, Mass., 1991

[45] T. Sanford. Hall Effect devices as Magnetometers in Cryogenic Applications, National Aeronautics and Space Administration, 1964

[46] Hall Generator MODEL 5080 Manual, F.W Bell.

[47] Abaqus Software Documentation. ABAQUS, Inc., Rising Sun Mills, 166 Valley Street, Providence, RI 02909-2499, USA. 


\section{Appendix A: Modeling Software}

\section{A.1 ABAQUS ${ }^{\circledR}$ Software}

ABAQUS is used for modeling the geometry of the structure and the defect. Applying a load to the structure will change the stress/strain fields and this in turn results in changing the parameters of the defect. The new model will be sent to MagNet software to analyze the magnetic flux field.

The various modules used in ABAQUS software are [47]:

PART module: In this step, a geometrical part is created for analysis. The part or various parts that have to be assembled is made using the various user-interactive tools.

PROPERTY module: In this step, sections are created with desired material properties and then one section or more than one section is assigned to the part created.

ASSEMBLY module: In this step, the various parts made are assembled according to the requirement or design of the component.

STEP module: In this step, the analysis step is defined. We determine which analysis needs to be considered, maximum and minimum load increments and the number of increments in the analysis.

INTERACTION module: In this step, mechanical interactions between regions of the model or interactions between the model and its surroundings are defined.

LOAD module: In this step, the load and the boundary conditions are applied to the structure. Load and boundary conditions are step-dependant criteria, which means that they need to be applied to the analysis steps in which they are defined.

MESH module: In this step. The mesh is generated on the assembly. 
The assembly is divided into many elements which are connected to each other by nodes. Element types and properties need to be set in the model.

JOB module: In this step, we create a job and run the analysis on the model with respect to the load and boundary conditions.

VISUALIZATION module: In this step, we can see the results of the finite element analysis in three dimensions by using a graphical display of the finite element model and results. This module obtains the model and the output information from the output database which works according to the output requests given in the step module.

\section{A.2 MATLAB ${ }^{\circledR}$ : Neural Network Tool Box}

The Neural Network Toolbox in Matlab can be used to design the neural network proposed to solve the electromagnetic inverse problem. Magnetic flux finite element models will be used to train the neural network. Matlab Neural Network Tool Box (MNNTB) contains many functions that can be used in the design of the network depending on the application and number of training models.

In Matlab, the graphical user interface (GUI) is designed to be simple and user friendly. Once the Network/Data Manager is up and running, the network can be created, viewed, trained, simulate it and export the final results to the workspace. Similarly, the data can be imported from the workspace for use in the graphical user interface.

Before training, it is often useful to scale the inputs and targets so that they always fall within a specified range. The function premnmx can be used to scale inputs and targets so that they fall in the range $[-1,1][18]$.

Another approach for scaling network inputs and targets is to normalize the mean and standard deviation of the training set. This procedure is implemented in the function 
prestd. It normalizes the inputs and targets so that they will have zero mean and unity standard deviation.

In some situations, the dimension of the input vector is large, but the components of the vectors are highly correlated (redundant). It is useful in this situation to reduce the dimension of the input vectors. An effective procedure for performing this operation is principal component analysis. This technique has three effects: it orthogonalizes the components of the input vectors (so that they are uncorrelated with each other); it orders the resulting orthogonal components (principal components) so that those with the largest variations come first; and it eliminates those components that contribute the least to the variation in the data set [18].

The performance of a trained network can be measured to some extent by the errors on the training, validation and test sets, but it is often useful to investigate the network response in more detail. One option is to perform a regression analysis between the network response and the corresponding targets. The routine postreg is designed to perform this analysis. 


\section{Appendix B: MATLAB ${ }^{\circledR}$ Code}

\section{"crackAnalysis_Cancellation"}

This code calculates the magnetic field produced by the crack and the plate edges after using the dummy plate design.

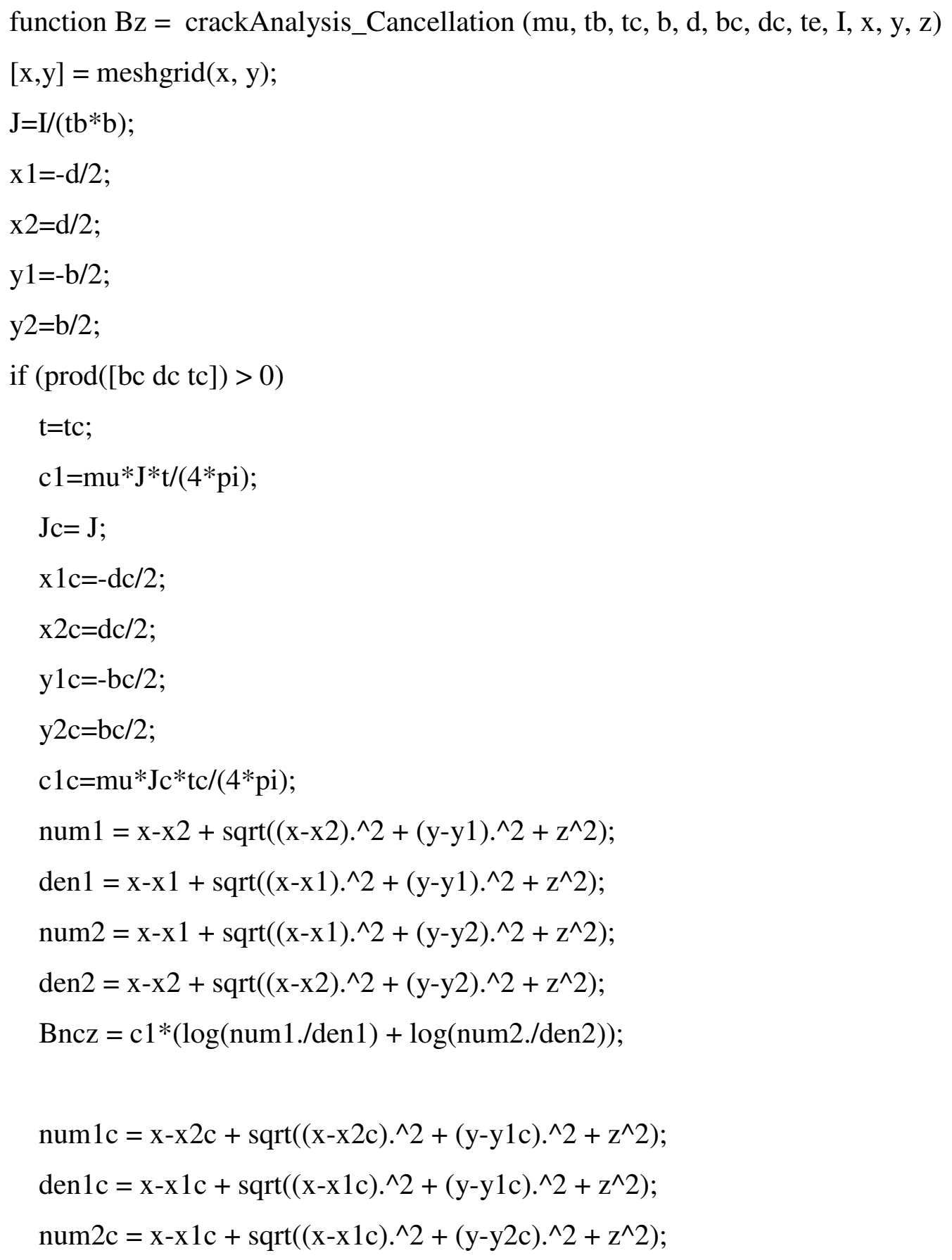




$$
\begin{aligned}
& \operatorname{den} 2 \mathrm{c}=\mathrm{x}-\mathrm{x} 2 \mathrm{c}+\operatorname{sqrt}\left((\mathrm{x}-\mathrm{x} 2 \mathrm{c}) .^{\wedge} 2+(\mathrm{y}-\mathrm{y} 2 \mathrm{c}) .^{\wedge} 2+\mathrm{z}^{\wedge} 2\right) ; \\
& \mathrm{Bcz}=\mathrm{c} 1 \mathrm{c} *(\log (\text { num } 1 \mathrm{c} . / \mathrm{den} 1 \mathrm{c})+\log (\text { num } 2 \mathrm{c} . / \mathrm{den} 2 \mathrm{c})) \text {; } \\
& \text { end } \\
& \mathrm{zc}=\mathrm{tb}+\mathrm{te}+\mathrm{z} \\
& \operatorname{num} 1 \mathrm{cn}=\mathrm{x}-\mathrm{x} 2+\operatorname{sqrt}\left((\mathrm{x}-\mathrm{x} 2) \cdot{ }^{\wedge} 2+(\mathrm{y}-\mathrm{y} 1) \cdot{ }^{\wedge} 2+\mathrm{zc}^{\wedge} 2\right) ; \\
& \operatorname{den} 1 \mathrm{cn}=\mathrm{x}-\mathrm{x} 1+\operatorname{sqrt}\left((\mathrm{x}-\mathrm{x} 1) \cdot{ }^{\wedge} 2+(\mathrm{y}-\mathrm{y} 1) \cdot{ }^{\wedge} 2+\mathrm{zc}^{\wedge} 2\right) ; \\
& \operatorname{num} 2 \mathrm{cn}=\mathrm{x}-\mathrm{x} 1+\operatorname{sqrt}\left((\mathrm{x}-\mathrm{x} 1) \cdot{ }^{\wedge} 2+(\mathrm{y}-\mathrm{y} 2) \cdot{ }^{\wedge} 2+\mathrm{zc}^{\wedge} 2\right) ; \\
& \operatorname{den} 2 \mathrm{cn}=\mathrm{x}-\mathrm{x} 2+\operatorname{sqrt}\left((\mathrm{x}-\mathrm{x} 2) \cdot{ }^{\wedge} 2+(\mathrm{y}-\mathrm{y} 2) \cdot{ }^{\wedge} 2+\mathrm{zc}^{\wedge} 2\right) \\
& \mathrm{c} 1 \mathrm{cn}=\mathrm{mu} * \mathrm{~J} * \mathrm{tb} /(4 * \mathrm{pi}) \text {; }
\end{aligned}
$$$$
\text { Bzcn }=\mathrm{c} 1 \mathrm{cn} *(\log (\text { num } 1 \mathrm{cn} . / \mathrm{den} 1 \mathrm{cn})+\log (\text { num } 2 \mathrm{cn} . / \mathrm{den} 2 \mathrm{cn})) \text {; }
$$$$
\text { if }(\mathrm{tb}-\mathrm{tc}>0)
$$$$
\mathrm{tb}=\mathrm{tb}-\mathrm{tc}
$$$$
\mathrm{Jb}=\mathrm{J} \text {; }
$$

$\mathrm{x} 1 \mathrm{~b}=-\mathrm{d} / 2$;

$\mathrm{x} 2 \mathrm{~b}=\mathrm{d} / 2$;

$\mathrm{y} 1 \mathrm{~b}=-\mathrm{b} / 2$;

$\mathrm{y} 2 \mathrm{~b}=\mathrm{b} / 2$;

$\mathrm{c} 1 \mathrm{~b}=\mathrm{mu} * \mathrm{Jb} * \mathrm{tb} /(4 * \mathrm{pi}) ;$

$\mathrm{zb}=\mathrm{tc}+\mathrm{z}$

$$
\begin{aligned}
& \text { num } 1 b=x-x 2 b+\operatorname{sqrt}\left((x-x 2 b) .^{\wedge} 2+(y-y 1 b) .^{\wedge} 2+z^{\wedge} 2\right) \\
& \operatorname{den} 1 b=x-x 1 b+\operatorname{sqrt}\left((x-x 1 b) . \wedge 2+(y-y 1 b) .^{\wedge} 2+z^{\wedge} 2\right) ; \\
& \text { num } 2 b=x-x 1 b+\operatorname{sqrt}\left((x-x 1 b) .^{\wedge} 2+(y-y 2 b) .^{\wedge} 2+z^{\wedge} 2\right) ; \\
& \operatorname{den} 2 b=x-x 2 b+\operatorname{sqrt}\left((x-x 2 b) .^{\wedge} 2+(y-y 2 b) .^{\wedge} 2+z^{\wedge} 2\right) \\
& \mathrm{Bzb}=\mathrm{c} 1 \mathrm{~b} *(\log (\text { num } 1 \mathrm{~b} . / \operatorname{den} 1 \mathrm{~b})+\log (\text { num } 2 \mathrm{~b} . / \mathrm{den} 2 \mathrm{~b})) \text {; }
\end{aligned}
$$

if $(\operatorname{prod}([b c d c t c])>0)$

$$
\mathrm{Bz}=\mathrm{Bzb}+\mathrm{Bncz}-\mathrm{Bcz}-\mathrm{Bzcn} ;
$$

else

$$
\mathrm{Bz}=\mathrm{Bzb}-\mathrm{Bzcn} ;
$$


end

else

$$
\mathrm{Bz}=\mathrm{Bncz-Bcz-Bzcn}
$$

end

\section{“crackAnalysis.m"}

This file carries out the magnetic field analysis for the magnetic field produced by a current flowing in a non-ferromagnetic plate. The magnetic field is a result of the plate edges and the crack. The dummy plate design is not a factor in this analysis.

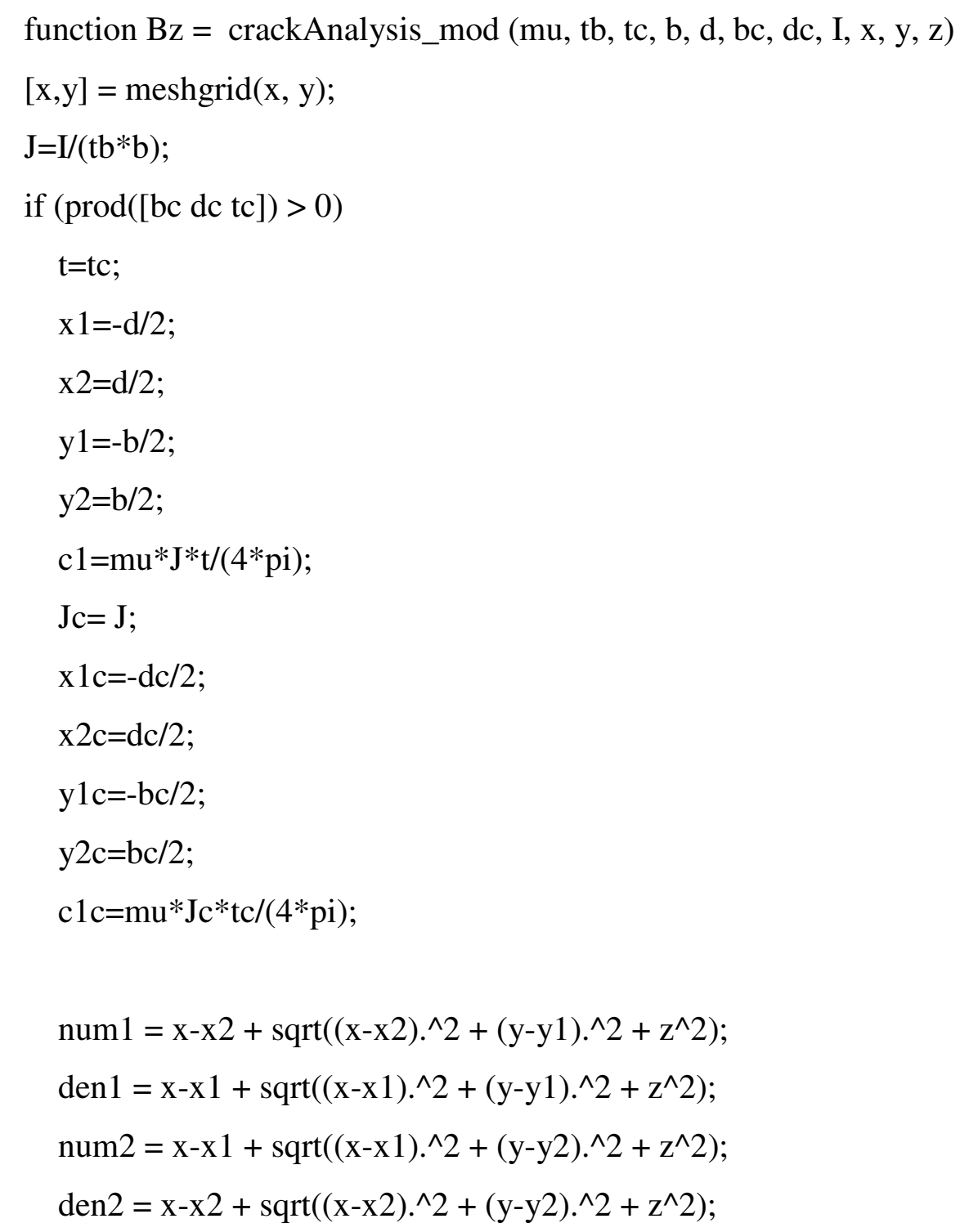




$$
\begin{aligned}
& \mathrm{Bncz}=\mathrm{c} 1 *(\log (\text { num } 1 . / \mathrm{den} 1)+\log (\text { num } 2 . / \mathrm{den} 2)) \\
& \operatorname{num} 1 \mathrm{c}=\mathrm{x}-\mathrm{x} 2 \mathrm{c}+\operatorname{sqrt}\left((\mathrm{x}-\mathrm{x} 2 \mathrm{c}) .^{\wedge} 2+(\mathrm{y}-\mathrm{y} 1 \mathrm{c}) .^{\wedge} 2+\mathrm{z}^{\wedge} 2\right) \\
& \operatorname{den} 1 \mathrm{c}=\mathrm{x}-\mathrm{x} 1 \mathrm{c}+\operatorname{sqrt}\left((\mathrm{x}-\mathrm{x} 1 \mathrm{c}) .^{\wedge} 2+(\mathrm{y}-\mathrm{y} 1 \mathrm{c}) .^{\wedge} 2+\mathrm{z}^{\wedge} 2\right) ; \\
& \operatorname{num} 2 \mathrm{c}=\mathrm{x}-\mathrm{x} 1 \mathrm{c}+\operatorname{sqrt}\left((\mathrm{x}-\mathrm{x} 1 \mathrm{c}) .^{\wedge} 2+(\mathrm{y}-\mathrm{y} 2 \mathrm{c}) .^{\wedge} 2+\mathrm{z}^{\wedge} 2\right) \\
& \operatorname{den} 2 c=x-x 2 c+\operatorname{sqrt}\left((x-x 2 c) \cdot{ }^{\wedge} 2+(y-y 2 c) \cdot{ }^{\wedge} 2+z^{\wedge} 2\right) ; \\
& \mathrm{Bcz}=\mathrm{c} 1 \mathrm{c}^{*}(\log (\text { num } 1 \mathrm{c} . / \mathrm{den} 1 \mathrm{c})+\log (\text { num } 2 \mathrm{c} . / \mathrm{den} 2 \mathrm{c})) \text {; }
\end{aligned}
$$

else

end

if $(\mathrm{tb}-\mathrm{tc}>0)$

$\mathrm{tb}=\mathrm{tb}-\mathrm{tc}$;

$\mathrm{Jb}=\mathrm{J}$;

$\mathrm{x} 1 \mathrm{~b}=-\mathrm{d} / 2$;

$\mathrm{x} 2 \mathrm{~b}=\mathrm{d} / 2$;

$\mathrm{y} 1 \mathrm{~b}=-\mathrm{b} / 2$;

$\mathrm{y} 2 \mathrm{~b}=\mathrm{b} / 2$;

$\mathrm{c} 1 \mathrm{~b}=\mathrm{mu} * \mathrm{Jb} * \mathrm{tb} /(4 * \mathrm{pi})$;

$$
\mathrm{zb}=\mathrm{tc}+\mathrm{z} ;
$$

num $1 b=x-x 2 b+\operatorname{sqrt}\left((x-x 2 b) .^{\wedge} 2+(y-y 1 b) .^{\wedge} 2+z^{\wedge} 2\right) ;$

$\operatorname{den} 1 b=x-x 1 b+\operatorname{sqrt}\left((x-x 1 b) .^{\wedge} 2+(y-y 1 b) .^{\wedge} 2+z^{\wedge} 2\right) ;$

num $2 b=x-x 1 b+\operatorname{sqrt}\left((x-x 1 b){ }^{\wedge} 2+(y-y 2 b) .^{\wedge} 2+z^{\wedge} 2\right) ;$

$\operatorname{den} 2 b=x-x 2 b+\operatorname{sqrt}\left((x-x 2 b) \wedge^{\wedge} 2+(y-y 2 b) .^{\wedge} 2+z b^{\wedge} 2\right) ;$

$\mathrm{Bzb}=\mathrm{c} 1 \mathrm{~b} *(\log ($ num $1 \mathrm{~b} . / \mathrm{den} 1 \mathrm{~b})+\log ($ num $2 \mathrm{~b} . / \mathrm{den} 2 \mathrm{~b}))$;

if $(\operatorname{prod}([b c d c t c])>0)$

$$
\mathrm{Bz}=\mathrm{Bzb}+\mathrm{Bncz}-\mathrm{Bcz} ;
$$

else

$$
\mathrm{Bz}=\mathrm{Bzb} ;
$$

end

else 


$$
\mathrm{Bz}=\mathrm{Bncz-Bcz}
$$

end

\section{“crackAnalysis_mod"}

This code is a modified code from the crackanalysis.m code. This file carries out the magnetic field analysis of the magnetic field produced by a current flowing in a nonferromagnetic plate. This magnetic field is a result of the plate edges and the crack. The dummy plate design is not a factor in this analysis.

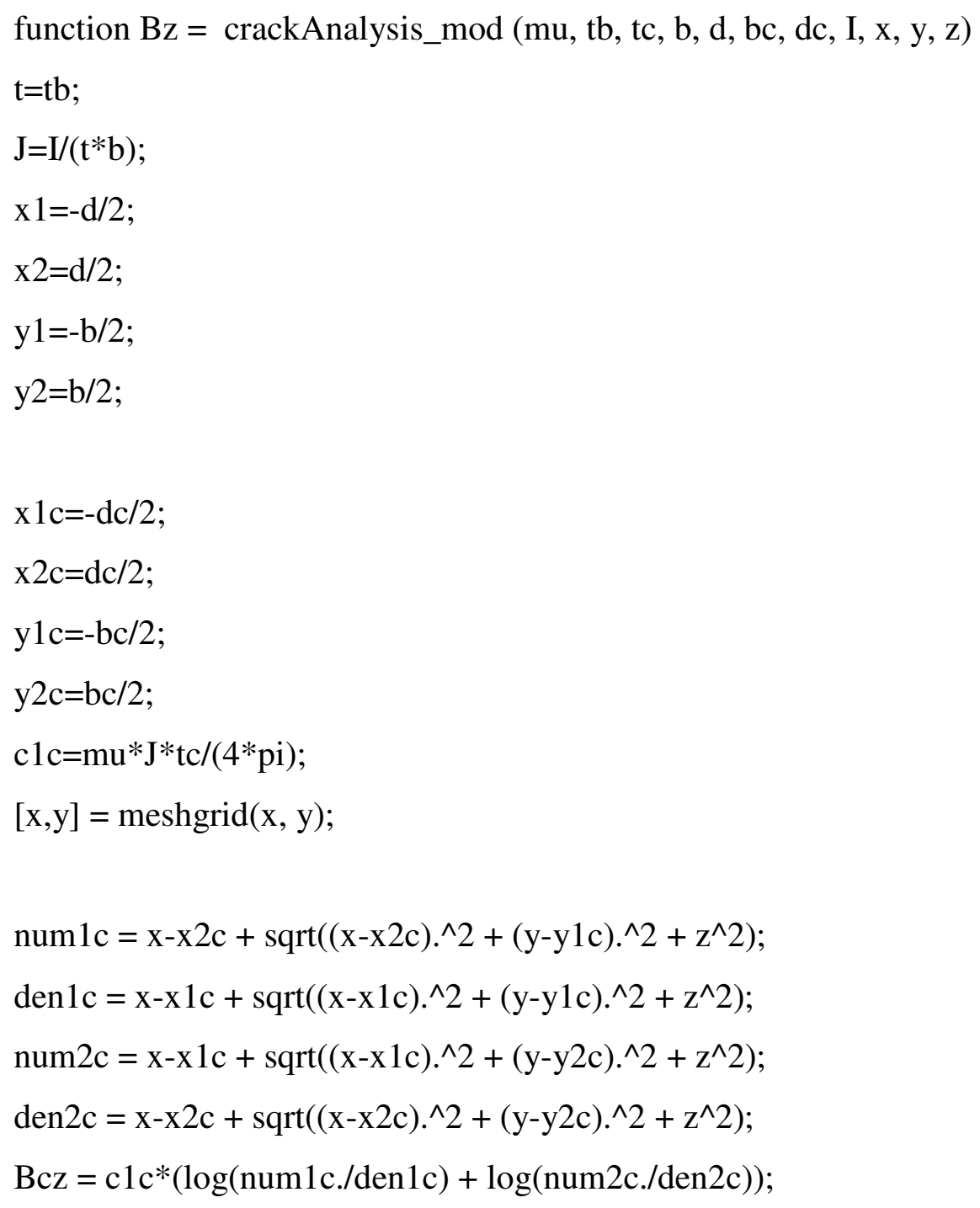




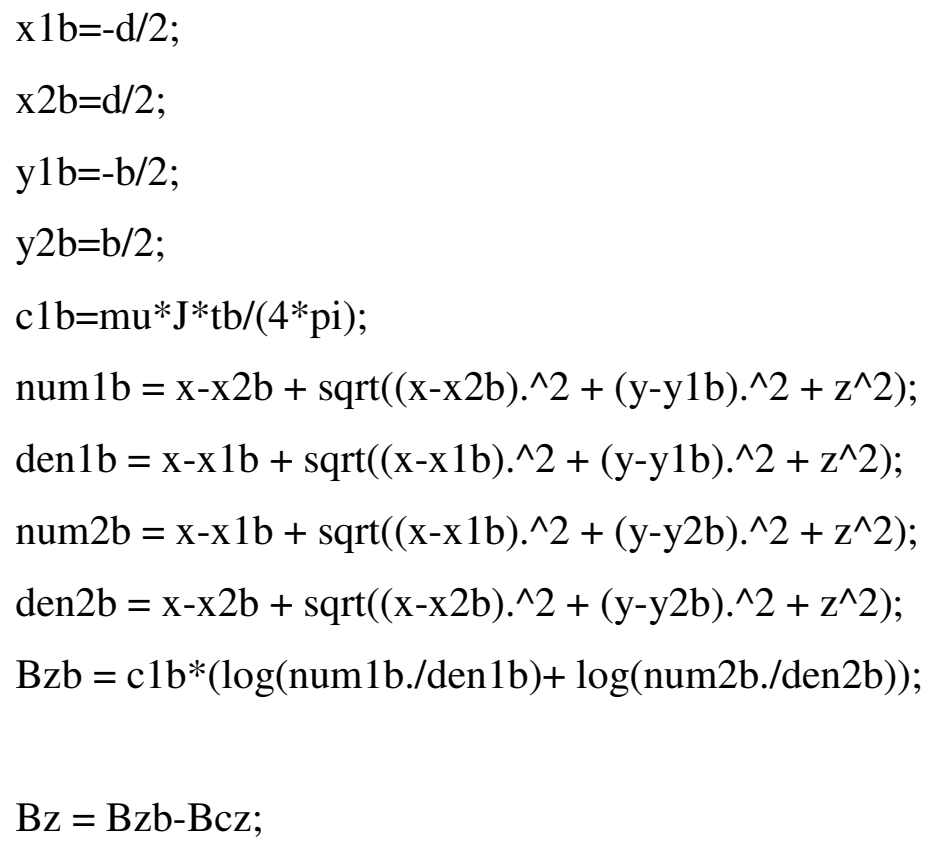

\section{"B_variations.m"}

This code studies the magnetic filed analysis for the plate specified in the program. This code addresses the effect of the crack profile factors on the magnetic filed produced by the crack at the crack tip.

$$
\begin{aligned}
& \mathrm{bp}=0.2 ; \\
& \mathrm{dp}=0.2 ; \\
& \mathrm{x} 1 \mathrm{p}=-\mathrm{dp} / 2 ; \\
& \mathrm{x} 2 \mathrm{p}=\mathrm{dp} / 2 ; \\
& \mathrm{y} 1 \mathrm{p}=-\mathrm{bp} / 2 ; \\
& \mathrm{y} 2 \mathrm{p}=\mathrm{bp} / 2 ; \\
& \mathrm{b}=0.008 ; \\
& \mathrm{d}=0.002 ; \\
& \mathrm{x}=0 ; \\
& \mathrm{x} 1=-\mathrm{d} / 2 ; \\
& \mathrm{x} 2=\mathrm{d} / 2 ;
\end{aligned}
$$




$$
\begin{aligned}
& \mathrm{y}=\mathrm{b} / 2 \text {; } \\
& \mathrm{y} 1=-\mathrm{b} / 2 \text {; } \\
& \mathrm{y} 2=\mathrm{b} / 2 \text {; } \\
& \mathrm{z}=\text { [0.0005: 0.0005: .005]; } \\
& d x 2=x-x 2 ; \\
& \mathrm{dx} 1=\mathrm{x}-\mathrm{x} 1 \text {; } \\
& \mathrm{dy} 2=\mathrm{y}-\mathrm{y} 2 \text {; } \\
& \mathrm{dy} 1=\mathrm{y}-\mathrm{y} 1 \text {; } \\
& \mathrm{dx} 2 \mathrm{p}=\mathrm{x}-\mathrm{x} 2 \mathrm{p} ; \\
& \mathrm{dx} 1 \mathrm{p}=\mathrm{x}-\mathrm{x} 1 \mathrm{p} \text {; } \\
& \mathrm{dy} 2 \mathrm{p}=\mathrm{y}-\mathrm{y} 2 \mathrm{p} ; \\
& \mathrm{dy} 1 \mathrm{p}=\mathrm{y}-\mathrm{y} 1 \mathrm{p} ; \\
& \mathrm{I}=10 \text {; } \\
& \text { muo }=4 * \text { pi*1e-7; } \\
& \mathrm{t}=0.002 \text {; }
\end{aligned}
$$

$$
\mathrm{Bp}=\mathrm{Jo} .{ }^{*} \mathrm{t}^{*} \mathrm{muo} /\left(4^{*} \mathrm{pi}\right) *\left(\operatorname { l o g } \left(\left(\mathrm{dx} 2 \mathrm{p}+\operatorname{sqrt}\left(\mathrm{dx} 2 \mathrm{p} .{ }^{\wedge} 2+\mathrm{dy} 1 \mathrm{p} .^{\wedge} 2+\mathrm{z} .^{\wedge} 2\right)\right) . /(\mathrm{dx} 1 \mathrm{p}+\right.\right.
$$
$\left.\left.\operatorname{sqrt}\left(\mathrm{dx} 1 \mathrm{p} .{ }^{\wedge} 2+\mathrm{dy} 1 \mathrm{p} \cdot{ }^{\wedge} 2+\mathrm{z} \cdot{ }^{\wedge} 2\right)\right)\right)+\ldots$

$$
\log \left(\left(\mathrm{dx} 1 \mathrm{p}+\operatorname{sqrt}\left(\mathrm{dx} 1 \mathrm{p} .^{\wedge} 2+\mathrm{dy} 2 \mathrm{p} .^{\wedge} 2+\mathrm{z} .^{\wedge} 2\right)\right) . /\left(\mathrm{dx} 2 \mathrm{p}+\operatorname{sqrt}\left(\mathrm{dx} 2 \mathrm{p} .^{\wedge} 2+\mathrm{dy} 2 \mathrm{p} .^{\wedge} 2\right.\right.\right.
$$
$\left.\left.\left.+\mathrm{z} .^{\wedge} 2\right)\right)\right)$;

$$
\mathrm{B}=\mathrm{Jo} \cdot{ }^{*} \mathrm{t}^{*} \mathrm{muo} /\left(4^{*} \mathrm{pi}\right) *\left(\operatorname { l o g } \left(\left(\mathrm{dx} 2+\operatorname{sqrt}\left(\mathrm{dx} 2 .^{\wedge} 2+\mathrm{dy} 1 .^{\wedge} 2+\mathrm{z}^{\wedge} 2\right)\right) . /(\mathrm{dx} 1+\right.\right.
$$
$\left.\left.\operatorname{sqrt}\left(\mathrm{dx} 1 .^{\wedge} 2+\mathrm{dy} 1 .^{\wedge} 2+\mathrm{z} .^{\wedge} 2\right)\right)\right)+\ldots$

$$
\log \left(\left(\mathrm{dx} 1+\operatorname{sqrt}\left(\mathrm{dx} 1 .^{\wedge} 2+\mathrm{dy} 2 .^{\wedge} 2+\mathrm{z} .^{\wedge} 2\right)\right) . /\left(\mathrm{dx} 2+\operatorname{sqrt}\left(\mathrm{dx} 2 .^{\wedge} 2+\mathrm{dy} 2 .^{\wedge} 2+\right.\right.\right.
$$
Z.^2))));

subplot(2,1,2), plot(z,abs(Bp-B),'o'); 


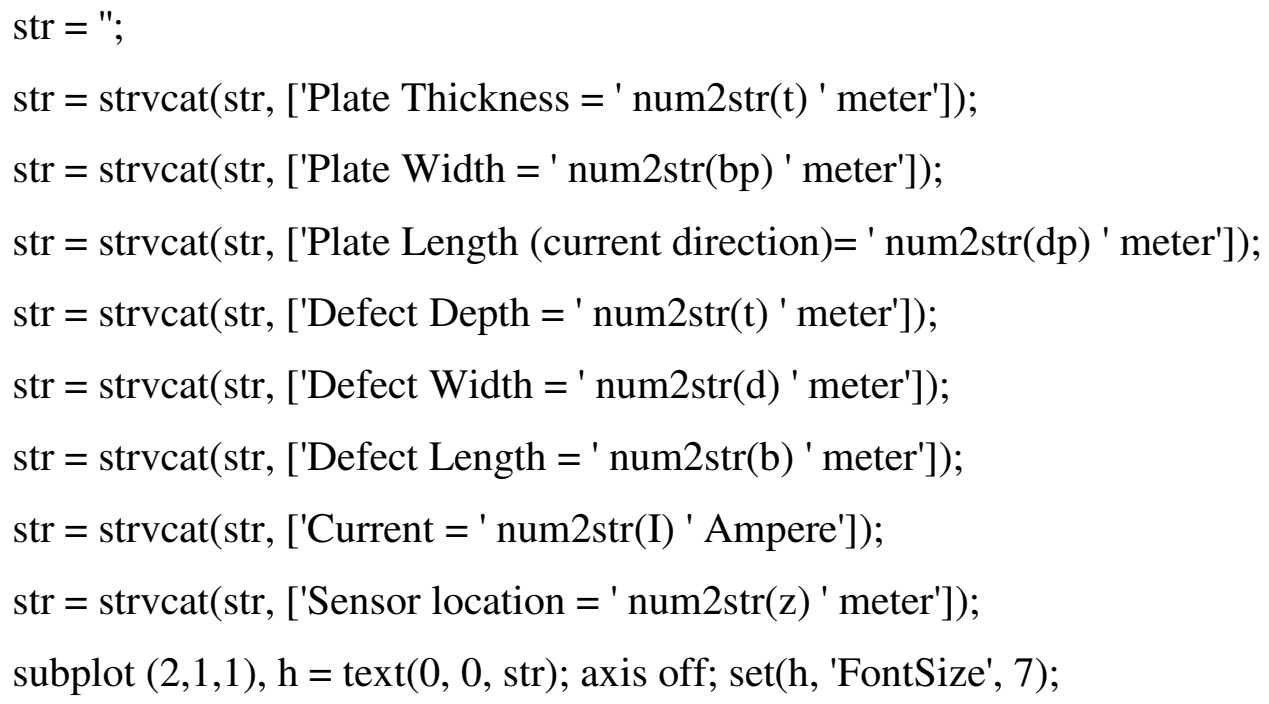

\section{"CrackAnalysisdataGeneration.m"}

This file takes the crack profile as an input, it calls the crack analysis file and generates a data file for the magnetic fields to train the neural networks.

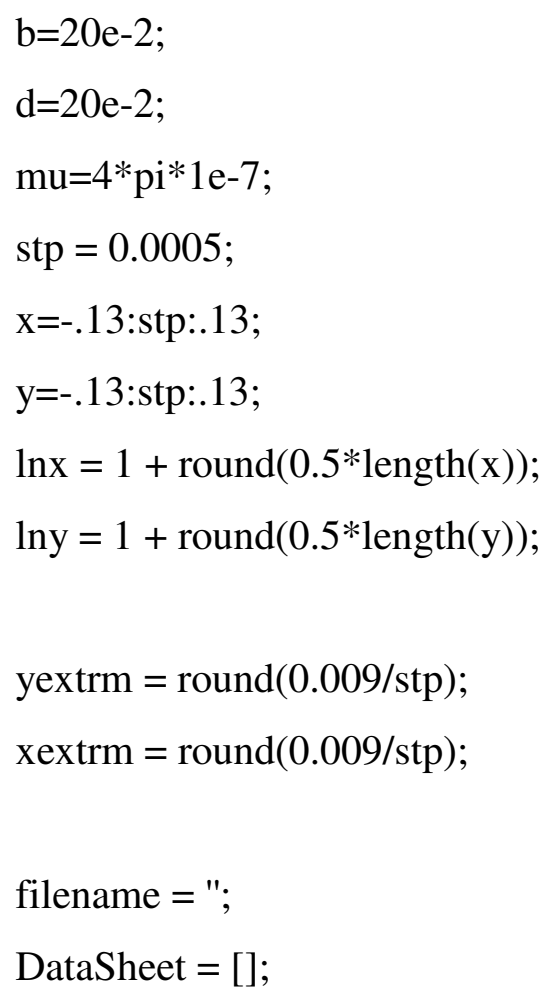

filename = ";

DataSheet $=[]$; 
while(isempty(filename))

[filename, path, ndx] = uigetfile('*.xl*', 'Select Modeling Parameters Sheet'); end

$\operatorname{if}(\operatorname{ndx}>0)$

[pt nm xt vn] = fileparts(filename);

[success, message, messageid $]=\operatorname{mkdir}($ path, $\mathrm{nm})$;

filename $=$ fullfile $($ path, filename $)$;

[Data,Header]=xlsread(filename);

for $\mathrm{i}=1$ :size $($ Data, 1$)$

$\mathrm{I}=\operatorname{Data}(\mathrm{i}, 1)$;

$\mathrm{z}=\operatorname{Data}(\mathrm{i}, 2)$;

$\mathrm{tb}=\operatorname{Data}(\mathrm{i}, 3)$;

$\mathrm{bc}=\operatorname{Data}(\mathrm{i}, 4)$

$\mathrm{dc}=\operatorname{Data}(\mathrm{i}, 5)$

tc $=$ Data $(\mathrm{i}, 6)$;

te $=0.5 \mathrm{e}-3$;

$\% \quad \mathrm{Bz}=$ crackAnalysis $(\mathrm{mu}, \mathrm{tb}, \mathrm{tc}, \mathrm{b}, \mathrm{d}, \mathrm{bc}, \mathrm{dc}, \mathrm{I}, \mathrm{x}, \mathrm{y}, \mathrm{z})$;

$\mathrm{Bz}=$ crackAnalysis_Cancellation $(\mathrm{mu}, \mathrm{tb}, \mathrm{tc}, \mathrm{b}, \mathrm{d}, \mathrm{bc}, \mathrm{dc}, \mathrm{te}, \mathrm{I}, \mathrm{x}, \mathrm{y}, \mathrm{z})$;

$\mathrm{Bdz}=\mathrm{Bz}(\ln x-\mathrm{x}$ extrm:lnx+xextrm, lny-yextrm:lny+yextrm $) ;$

$\operatorname{mxBdz}=\max (\max (B d z))$

$\mathrm{h}=$ figure;

set (h, 'Position', [1 31 1024 662]);

hp = subplot('Position', [0.061 0.5380 .2710 .387$]$ );

subplot (hp), mesh(x,y,Bz'), xlabel('Y'), ylabel('X'), zlabel('B_z'), title ('Magnetic Flux Leakage', 'FontSize', 8);

hp = subplot('Position', [0.40 0.534 0.23 0.391]); 
subplot $(\mathrm{hp}), \quad[\mathrm{cnt}, \mathrm{hndl}]=\operatorname{contour}(\mathrm{x}(\ln \mathrm{x}-\mathrm{x}$ extrm:lnx $+\mathrm{xextrm}), \mathrm{y}(\ln \mathrm{y}-$ yextrm:Iny+yextrm),Bdz'); colorbar, ,...

xlabel('Y'), ylabel('X'), title ('Magnetic Flux Leakage Contour around defect', 'FontSize', 8);

hp = subplot('Position', [0.692 0.475 0.276 0.45]);

subplot (hp), mesh(x(lnx-xextrm:lnx+xextrm), y(lny-yextrm:lny+yextrm)

,Bdz'),...

xlabel('Y'), ylabel('X'), zlabel('B_z'), title ('Magnetic Flux Leakage around defect', 'FontSize', 8);

hp = subplot('Position', [ [ 0.130 .110 .517 0.341]);

subplot (hp), plot(x(lnx-xextrm:lnx+xextrm), $\left.(\operatorname{Bdz}(:, \text { yextrm }))^{\prime}\right), \ldots$

xlabel('Y'), ylabel('B_z Profile'), ht $=$ text $(.7 * x(\ln x-x e x t r m)$, $1.4 * \min (\operatorname{Bdz}(:, y e x t r m))$, 'Profile of Magnetic Flux Leakage around defect normal to direction of I @ $\left.\mathrm{x}=0^{\prime}\right)$;

set(ht, 'FontSize', 8); axis([x(lnx-xextrm) $\mathrm{x}(\ln x+x \operatorname{extrm}) \quad \min (\mathrm{Bdz}(:$, yextrm)) $\max (\operatorname{Bdz}($, , yextrm) $)])$;

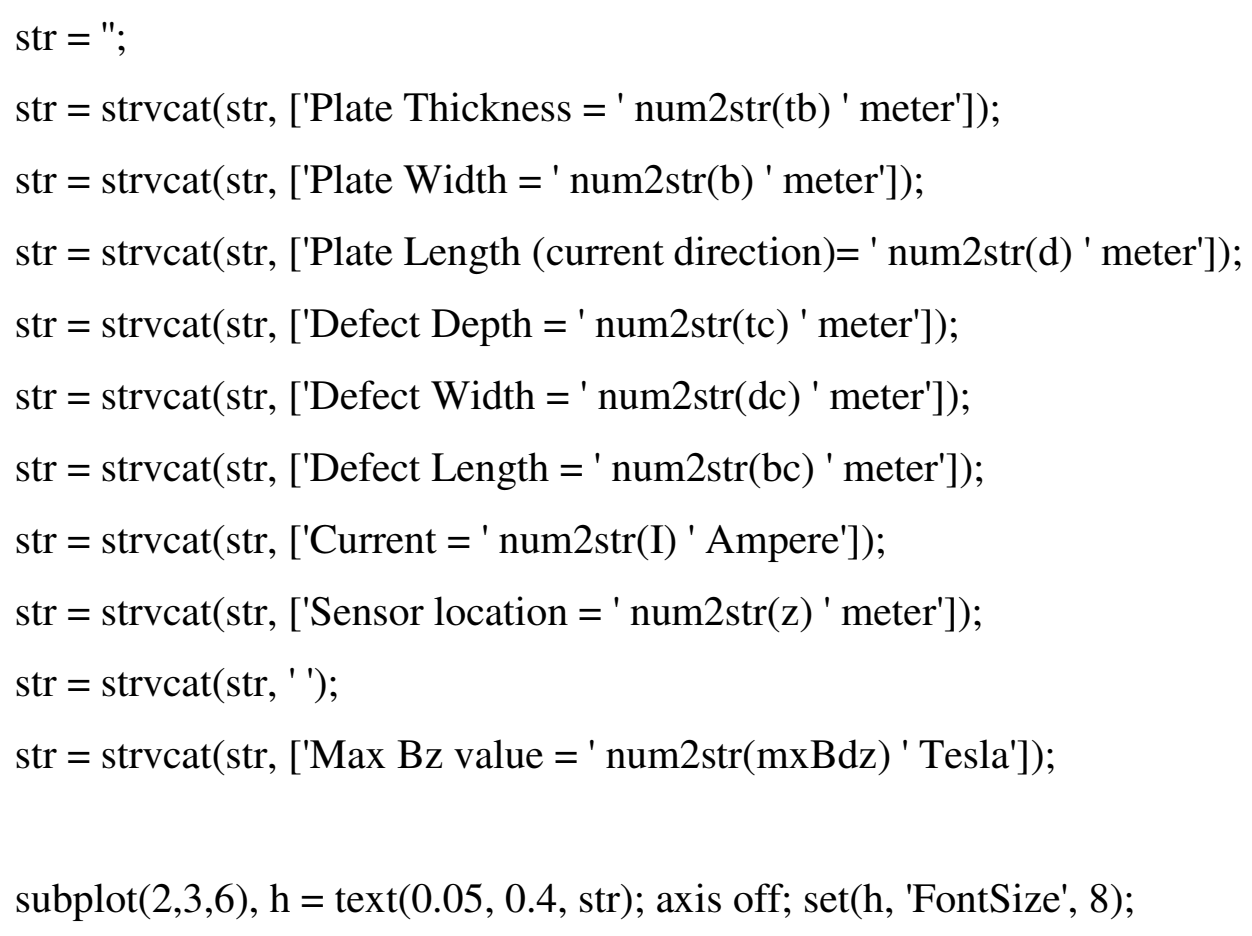




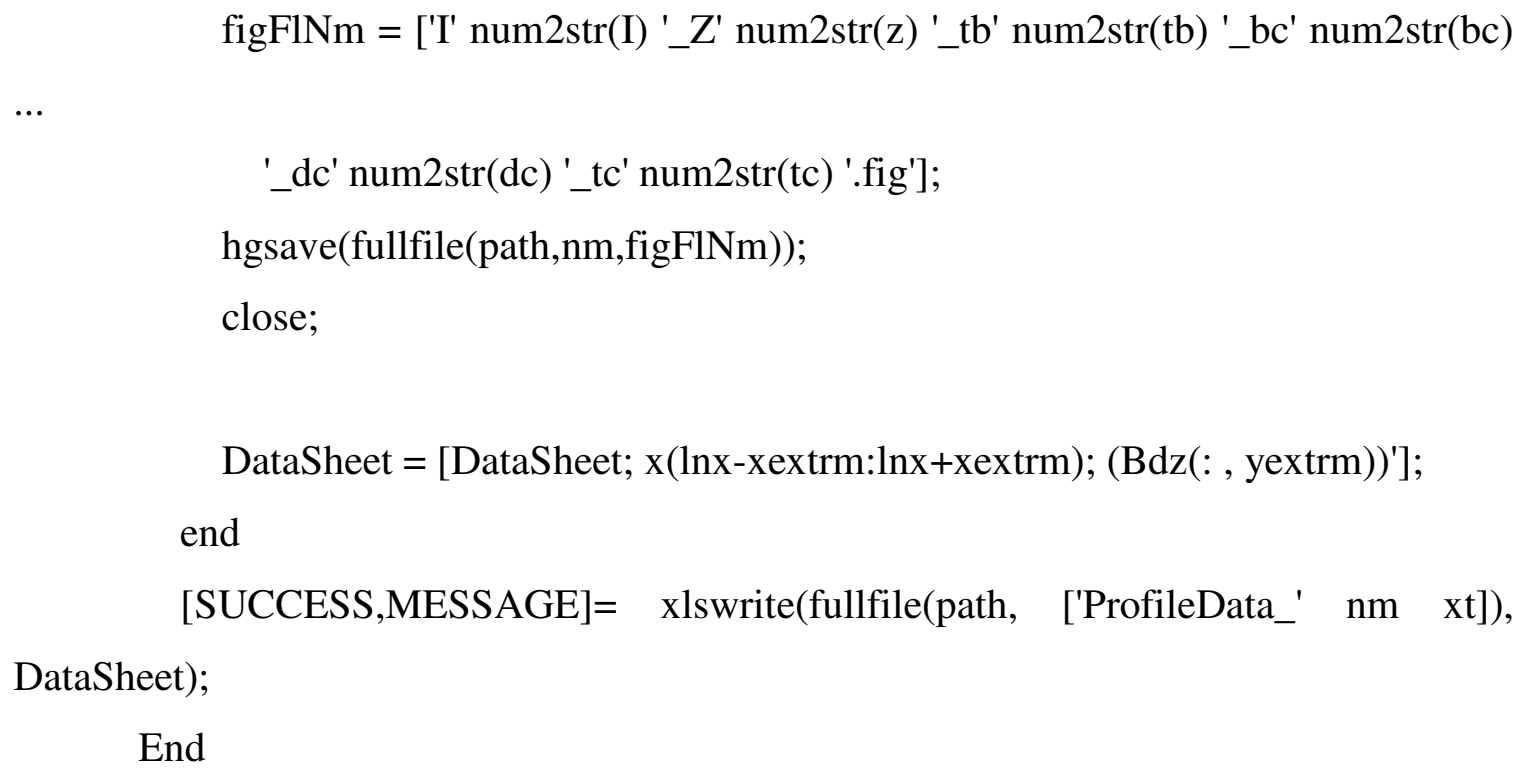

\section{“CrackAnalysisdatagenerationExpcomp.m”}

This file takes the crack profile as an input, it calls the crack analysis file and generates a data file for the magnetic fields related to the experimental work.

$$
\begin{aligned}
& \mathrm{b}=0.1524 ; \\
& \mathrm{d}=0.2286 ; \\
& \mathrm{mu}=4 * \mathrm{pi}^{*} 1 \mathrm{e}-7 ; \\
& \mathrm{stp}=0.0005 ; \\
& \mathrm{x}=-.15: \mathrm{stp}: .15 ; \\
& \mathrm{y}=-.15: \mathrm{stp}: .15 ; \\
& \% \text { del }=0.2 ; \\
& \% \mathrm{x}=-(1+\mathrm{del}) * \mathrm{~d} / 2: \mathrm{stp}:(1+\mathrm{del}) * \mathrm{~d} / 2 ; \\
& \% \mathrm{y}=-(1+\mathrm{del}) * \mathrm{~b} / 2: \mathrm{stp}:(1+\mathrm{del}) * \mathrm{~b} / 2 ; \\
& \ln \mathrm{x}=1+\operatorname{round}(0.5 * \operatorname{length}(\mathrm{x})) ; \\
& \operatorname{lny}=1+\operatorname{round}(0.5 * \operatorname{length}(\mathrm{y})) ; \\
& \text { yextrm = round }(0.009 / \mathrm{stp}) ; \\
& \text { xextrm = round }(0.009 / \mathrm{stp}) ;
\end{aligned}
$$




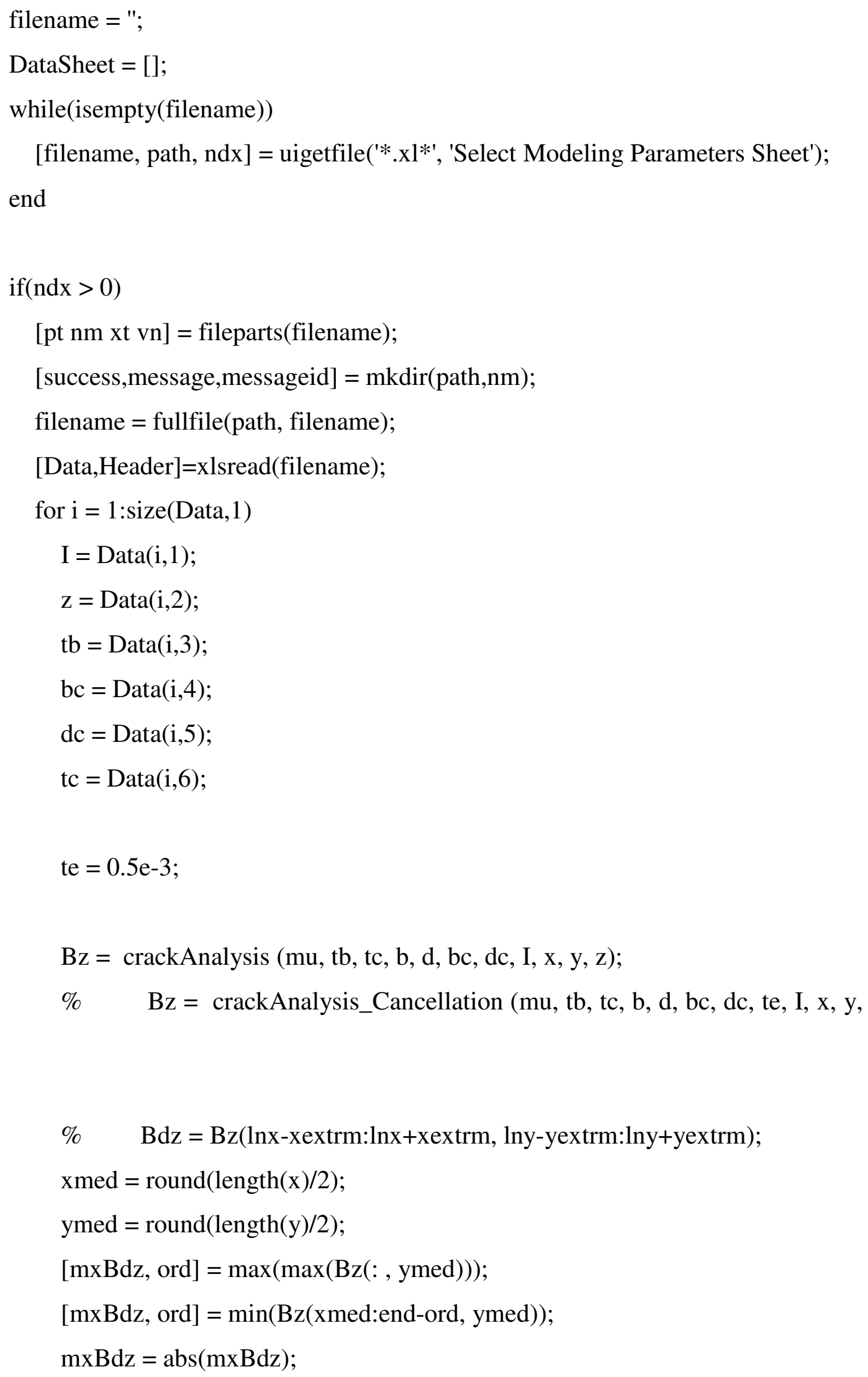




$$
\mathrm{Bdz}=\operatorname{Bz}(\max ([\mathrm{xmed}-\text { ord-15 1] }): \min ([\mathrm{xmed}+\text { ord }+15 \quad \text { length }(\mathrm{x})]),
$$

$\max ([y m e d-o r d-151]): \min ([y m e d+o r d+15$ length(y)]));

$\mathrm{h}=$ figure;

set (h, 'Position', [ [ 1311024 662]);

hp = subplot('Position', [0.061 0.538 0.271 0.387]);

subplot (hp), mesh(x,y,Bz'), xlabel('Y'), ylabel('X'), zlabel('B_z'), title ('Magnetic Flux Leakage', 'FontSize', 8);

hp = subplot('Position', [0.40 0.534 0.23 0.391]);

subplot $(\mathrm{hp}),[\mathrm{cnt}, \mathrm{hndl}]=\operatorname{contour}(\max ([\mathrm{xmed}-$ ord -151$]): \min ([\mathrm{xmed}+\mathrm{ord}+15$ length(x)]), $\max ([y m e d-o r d-151]): \min ([y m e d+o r d+15$ length(y)]), Bdz'); colorbar, ,...

xlabel('Y'), ylabel('X'), title ('Magnetic Flux Leakage Contour around defect', 'FontSize', 8);

hp = subplot('Position', [0.692 0.475 0.276 0.45]);

subplot (hp), $\operatorname{mesh}(\max ([x m e d-o r d-151]): \min ([x m e d+o r d+15$ length(x)]), $\max ([y m e d-o r d-151]): \min ([y m e d+o r d+15$ length(y)]),Bdz'),...

xlabel('Y'), ylabel('X'), zlabel('B_z'), title ('Magnetic Flux Leakage around defect', 'FontSize', 8);

hp = subplot('Position', [0.13 0.11 0.517 0.341]);

$\% \quad$ subplot $(\mathrm{hp}), \operatorname{plot}\left(\mathrm{x}(\ln \mathrm{x}-\mathrm{x}\right.$ extrm:Inx+xextrm $\left.),(\operatorname{Bdz}(:, \text { yextrm }))^{\prime}\right), \ldots$

$\% \quad$ xlabel('Y'), ylabel('B_z Profile'), ht = text $\left(.7^{*} x(\operatorname{lnx}-x e x t r m)\right.$, $1.4 * \min (\operatorname{Bdz}(:$, yextrm $))$, 'Profile of Magnetic Flux Leakage around defect normal to direction of I @ $\left.\mathrm{x}=0^{\prime}\right)$;

$\% \quad$ set(ht, 'FontSize', 8); axis([x(lnx-xextrm) $\mathrm{x}(\ln \mathrm{x}+\mathrm{x}$ extrm $) \min (\mathrm{Bdz}(:$, yextrm)) $\max (\operatorname{Bdz}(:, \operatorname{yextrm}))])$;

$\% \quad$ subplot $(\mathrm{hp}), \operatorname{plot}\left(\mathrm{x},(\mathrm{Bz}(:, \text { yextrm }))^{\prime}\right), \ldots$

$\% \quad$ xlabel('Y'), ylabel('B_z Profile'), ht $=$ text $(.7 * x(\operatorname{lnx}-x e x t r m)$, $1.4 * \min (\mathrm{Bz}(:$, yextrm $))$, 'Profile of Magnetic Flux Leakage around defect normal to direction of I @ $\left.\mathrm{x}=0^{\prime}\right)$; 
$\% \quad \operatorname{set}($ ht, 'FontSize', 8); axis([x(1) x(end) $\min (\mathrm{Bz}(:$, yextrm $)) \max (\mathrm{Bz}($ : , yextrm))]);

subplot (hp), plot(x, $\left.(\mathrm{Bz}(:, \text { ymed }))^{\prime}\right), \ldots$

xlabel('Y'), ylabel('B_z Profile'), ht = text $\left(.7^{*} x(\operatorname{lnx}-x \operatorname{extrm}), 1.4^{*} \min (\mathrm{Bz}(\right.$ : , ymed)), 'Profile of Magnetic Flux Leakage around defect normal to direction of I @ $\mathrm{x}=$ $\left.0^{\prime}\right)$;

set(ht, 'FontSize', 8); axis([x(1) x(end) $\min (\mathrm{Bz}($ : , ymed $)) \max (\mathrm{Bz}($ : , ymed))]);

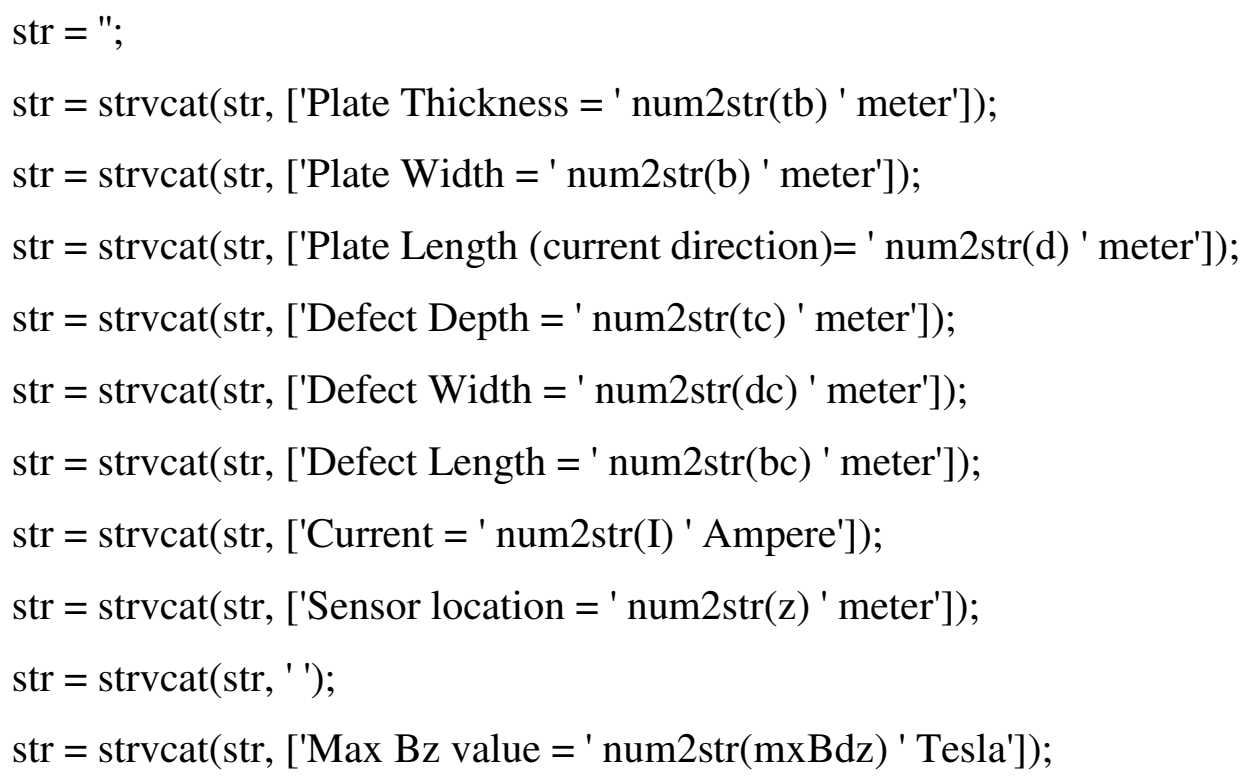

$\% \quad$ DataSheet $=\left[\right.$ DataSheet; $\left.x(\ln x-x \operatorname{extrm}: \ln x+x \operatorname{extrm}) ;(\operatorname{Bdz}(:, \text { yextrm }))^{\prime}\right]$; end 
$\% \quad$ [SUCCESS,MESSAGE]= xlswrite(fullfile(path, ['ProfileData_' nm xt]), DataSheet);

End

\section{“CrackNNTrain.m”}

This code is used to train the neural network; it contains the neural network design.

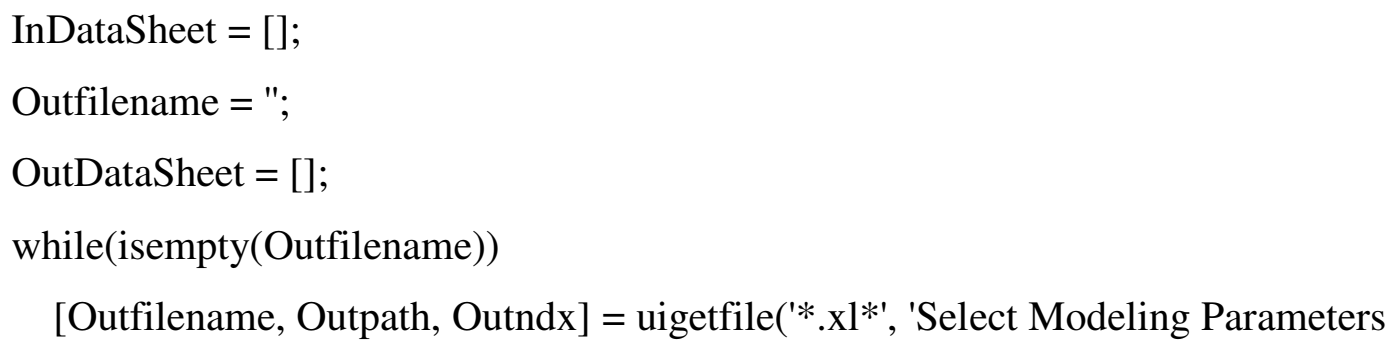
Sheet');

$$
\begin{aligned}
& \text { end } \\
& \text { if(Outndx > 0) } \\
& \text { filename = fullfile(Outpath, ['ProfileData_' Outfilename] }) \\
& \quad \text { [nData,InHeader]=xlsread(filename); } \\
& \text { filename = fullfile(Outpath, Outfilename); } \\
& \text { [OutData,OutHeader]=xlsread(filename); } \\
& \text { bc }=(\text { OutData }(:, 4))^{\prime} ; \\
& \text { dc }=(\text { OutData }(:, 5))^{\prime} ; \\
& \text { tc }=(\text { OutData }(:, 6))^{\prime} ; \\
& \text { Bz }=(\operatorname{InData}(2: 2: \text { end },:))^{\prime} ; \\
& \text { t }=[\text { bc; dc; tc]; }
\end{aligned}
$$$$
\text { [InData,InHeader]=xlsread(filename); }
$$$$
\text { [OutData,OutHeader]=xlsread(filename); }
$$ 


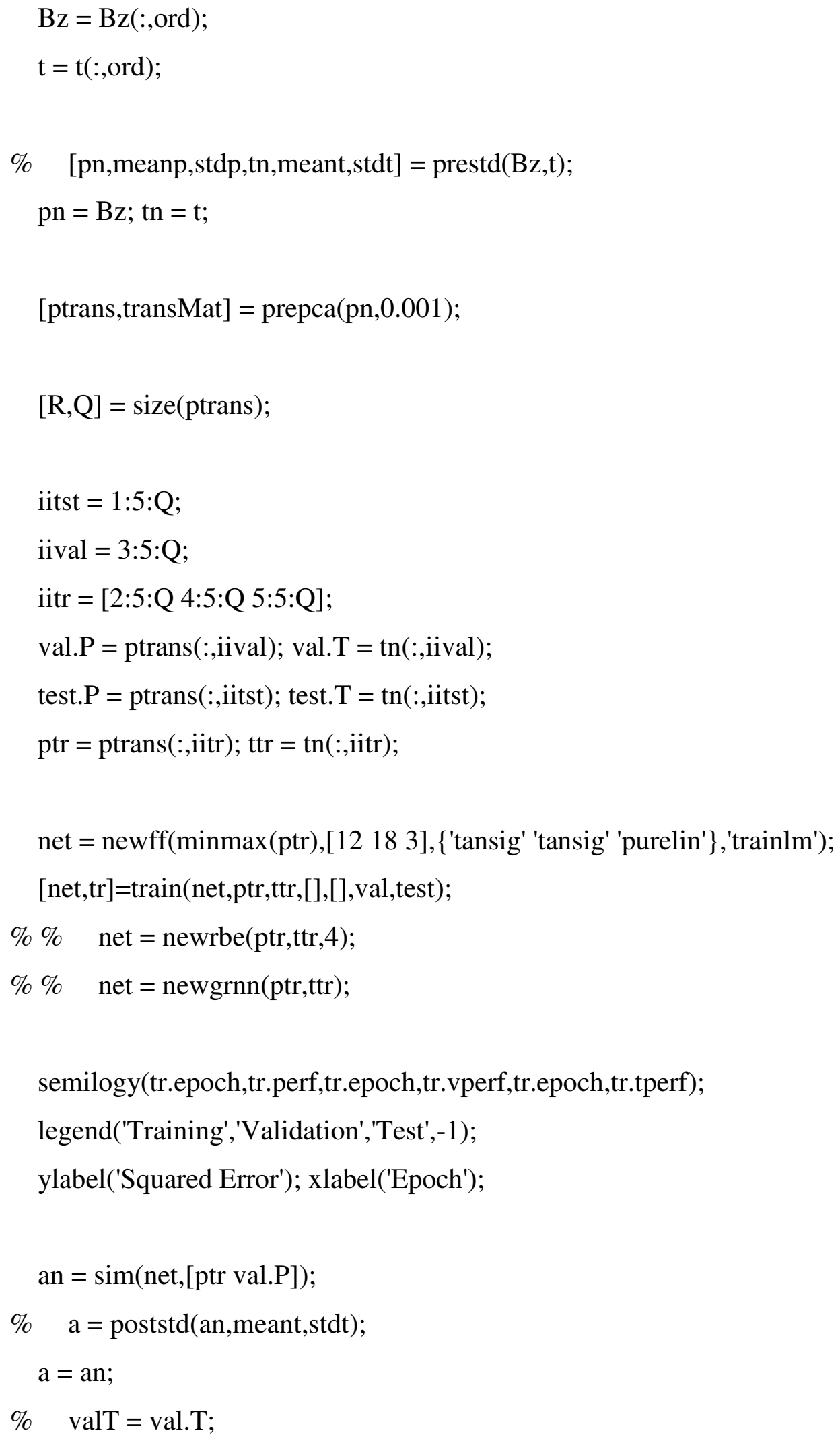




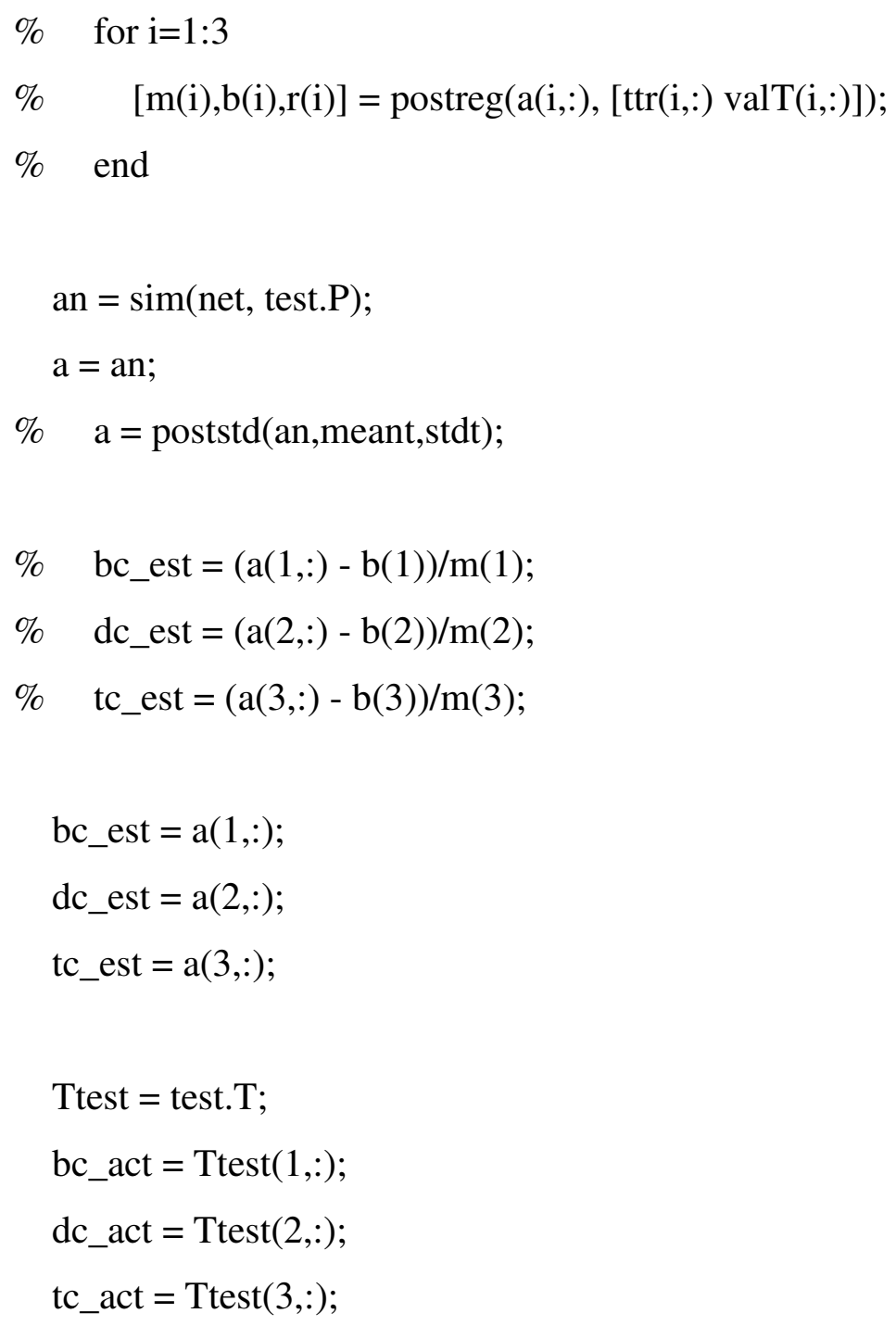

Err_bc $=100 *\left(b c \_e s t-b c \_a c t\right) . / b c \_a c t ;$ medAbsBcErr $=$ median $(\operatorname{abs}($ Err_bc $))$;

Err_dc $=100 *($ dc_est-dc_act $) . / d c \_a c t ;$ $\operatorname{medAbsDcErr}=\operatorname{median}(\operatorname{abs}($ Err_dc $))$;

Err_tc $=100^{*}\left(t c \_\right.$est-tc_act $) . / t c \_a c t ;$ medAbsTcErr $=$ median $(\operatorname{abs}($ Err_tc $))$;

figure, 


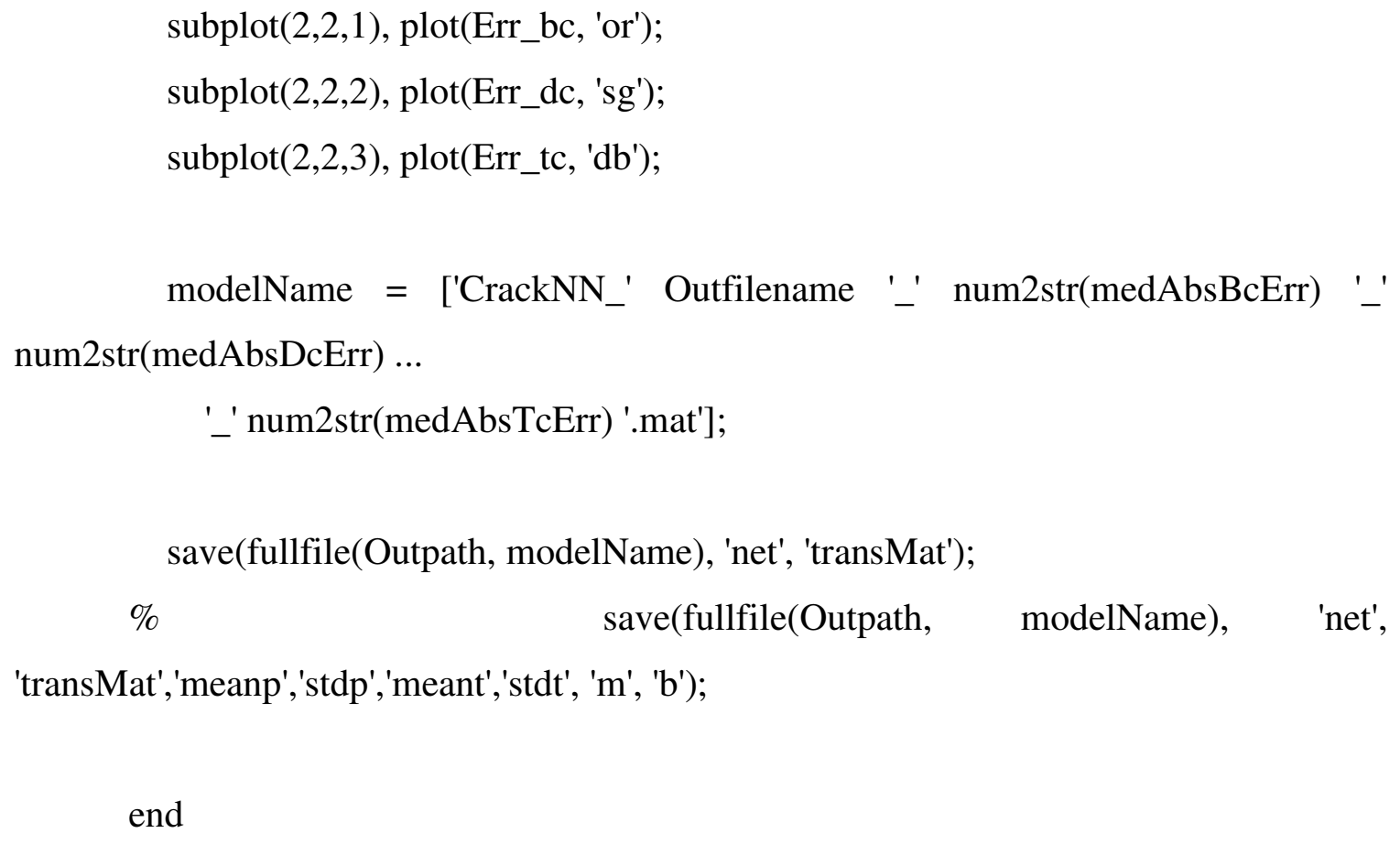

end

\section{" crackprofileprediction.m"}

This code is used to predict the crack profile. It calls the Network model and the crack profile file and gives the predicted crack profile.

netfilename $=$ ";

while (isempty(netfilename))

[netfilename, netpath, netndx] = uigetfile('CrackNN_*.mat', 'Select CrackNN Model');

end

DataSheet $=[]$;

filename $=$ ";

if(netndx $>0)$

while(isempty(filename))

[filename, path, $\mathrm{ndx}$ ] = uigetfile('*.xl*', 'Select Measurement Data Sheet'); end 


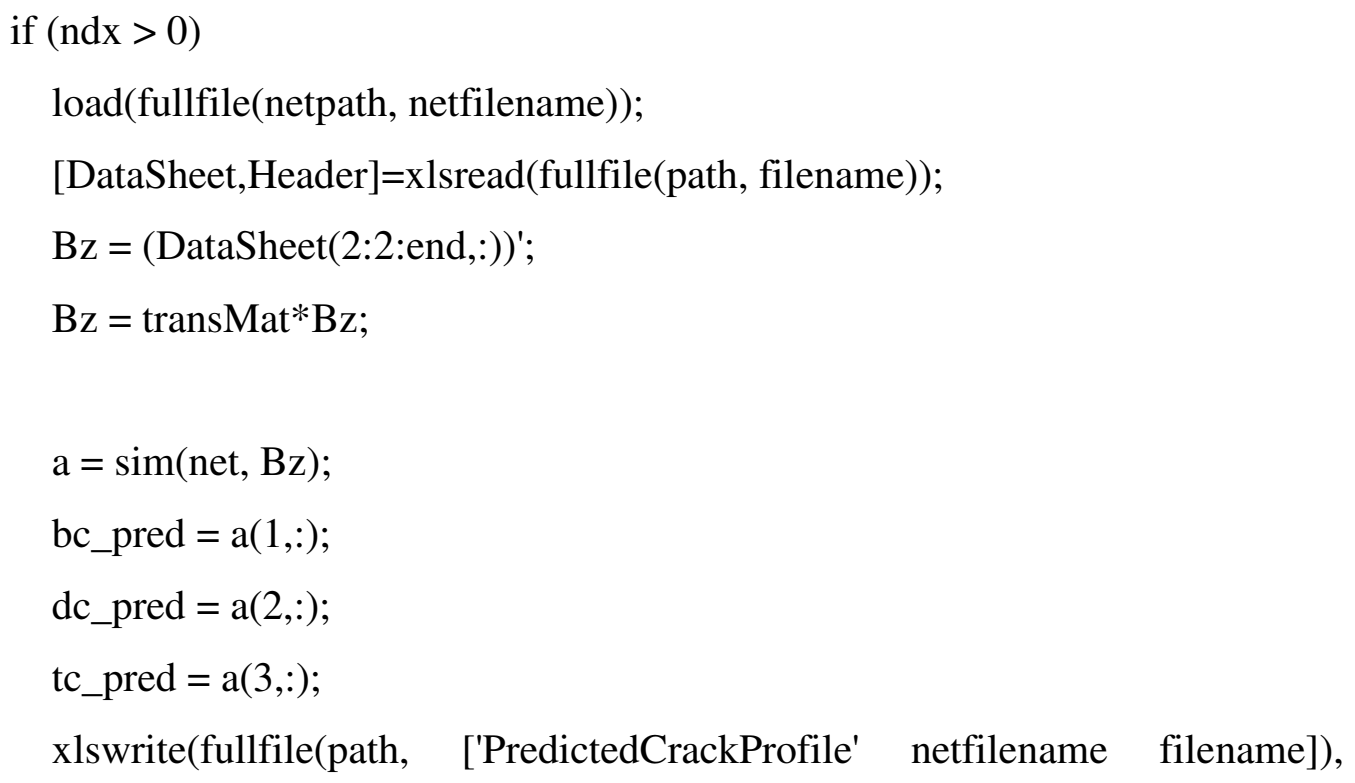
[bc_pred' dc_pred' tc_pred']);

end

end

\section{"Crackanalysisprofiletestdrive.m"}

This file tests the neural network with new crack profiles. It takes inputs from the sensor readings and predicts the crack profile.
$\%$ tc $=34 \mathrm{e}-6 / 3$;
$\% \mathrm{bc}=8 \mathrm{e}-3$;
$\% \mathrm{dc}=2 \mathrm{e}-3$;
$\% \mathrm{~b}=114 \mathrm{e}-3$;
$\% \mathrm{~d}=144 \mathrm{e}-3$
$\% \mathrm{I}=10$
$\% \mathrm{mu}=4 * \mathrm{pi} * 1 \mathrm{e}-7$
$\%$ stp $=0.0005$;
$\% \mathrm{x}=-.1$ :stp:.1;
$\% \mathrm{y}=-.1:$ stp:.1; 


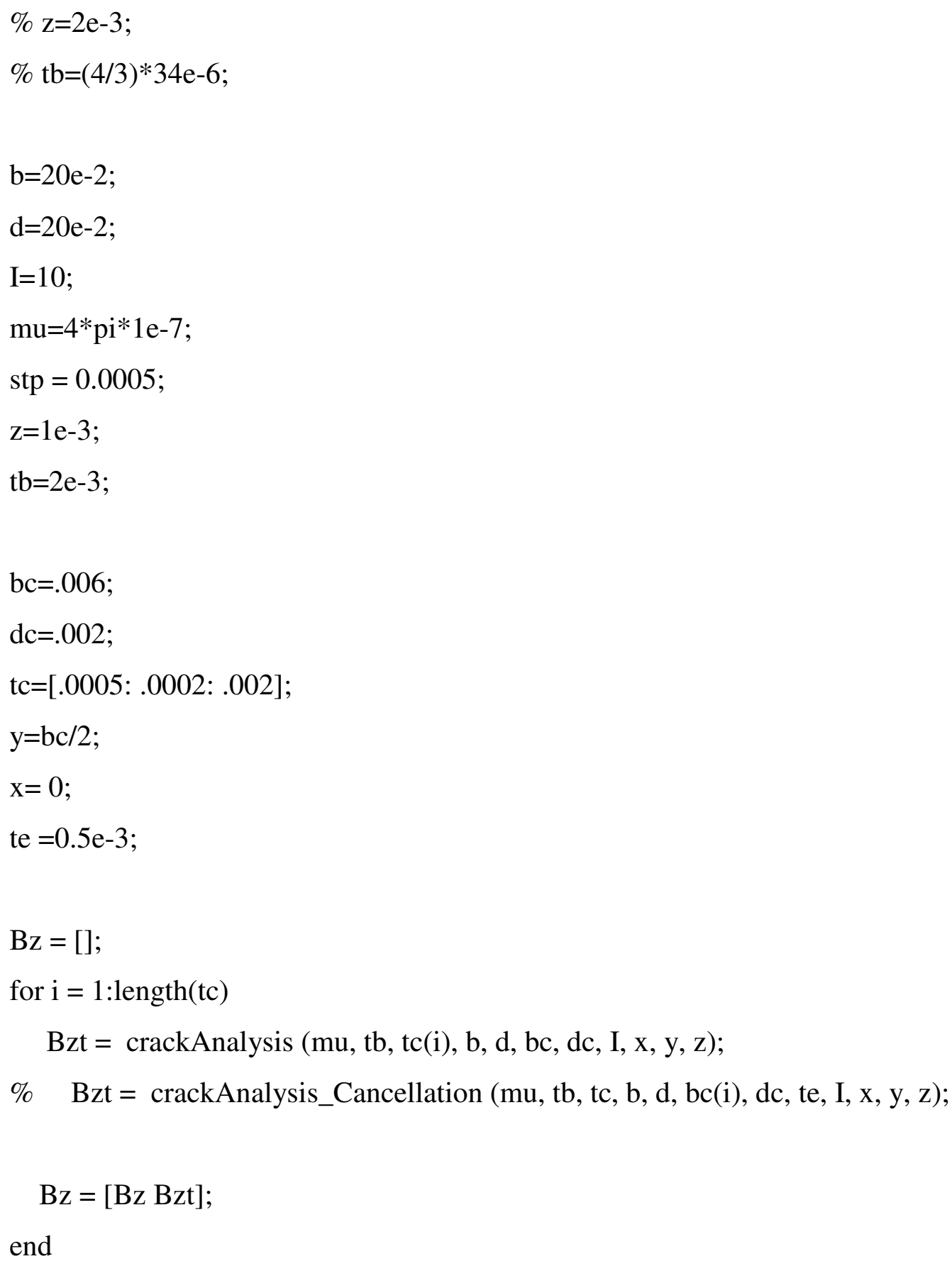




$$
\begin{aligned}
& \text { str }=\operatorname{strvcat}(\text { str, ['Plate Length }(\text { current direction })=\text { ' num2str(d) ' meter']); } \\
& \text { str }=\operatorname{strvcat}(\text { str, ['Defect Depth }=\text { ' num2str(tc) ' meter'] }) \text {; } \\
& \text { str }=\operatorname{strvcat}(\text { str, ['Defect Width = ' num2str(dc) ' meter']); } \\
& \operatorname{str}=\operatorname{strvcat}(\text { str, }[\text { 'Defect Length }=\text { ' num2str(bc) ' meter'] }) \text {; } \\
& \text { str }=\text { strvcat }(\text { str, ['Current }=\text { ' num2str(I) ' Ampere']); } \\
& \operatorname{str}=\operatorname{strvcat}(\mathrm{str},[\text { 'Sensor location }=\text { ' num2str(z) ' meter'] }) \text {; }
\end{aligned}
$$

subplot $(2,1,1), \mathrm{h}=\operatorname{text}(0,0, \mathrm{str})$; axis off; set(h, 'FontSize', 7$)$; 